\title{
AN EXAMINATION OF CULTURAL VALUES AND EMPLOYEES' PERCEPTIONS OF SUPPORT ON AFFECTIVE REACTION AND THE DESIRE TO PARTICIPATE IN A FORMAL MENTORING PROGRAM IN AN OILFIELD SERVICES CORPORATION
}

\author{
A Dissertation \\ by \\ HANNA BEA HAYES \\ Submitted to the Office of Graduate Studies of \\ Texas A\&M University \\ in partial fulfillment of the requirements for the degree of \\ DOCTOR OF PHILOSOPHY
}

May 2012

Major Subject: Education and Human Resource Development 


\title{
AN EXAMINATION OF CULTURAL VALUES AND EMPLOYEES' PERCEPTIONS \\ OF SUPPORT ON AFFECTIVE REACTION AND THE DESIRE TO PARTICIPATE IN \\ A FORMAL MENTORING PROGRAM IN AN OILFIELD SERVICES CORPORATION
}

\author{
A Dissertation \\ by \\ HANNA BEA HAYES \\ Submitted to the Office of Graduate Studies of \\ Texas A\&M University \\ in partial fulfillment of the requirements for the degree of \\ DOCTOR OF PHILOSOPHY
}

Approved by:

Chair of Committee, $\quad$ Larry M. Dooley

Committee Members, Toby Marshall Egan

Homer Tolson

James Lindner

Head of Department, $\quad$ Frederick Nafuko

May 2012

Major Subject: Education and Human Resource Development 


\begin{abstract}
An Examination of Cultural Values and Employees' Perceptions of Support on Affective Reaction and the Desire to Participate in a Formal Mentoring Program in an Oilfield Services Corporation. (May 2012)

Hanna Bea Hayes, B.S., Texas A\&M University; M.S., University of Texas at Dallas Chair of Advisory Committee: Dr. Larry M. Dooley
\end{abstract}

Many researchers have examined the effect of formal mentoring on job satisfaction and organizational commitment. However, there has been little or no focus on an employee's intent to participate in a formal mentoring program based upon an employee's perceived organizational support, and/or affective reaction (job satisfaction and organizational commitment). In the current study, the researcher examined the relationship among cultural values, perceived organizational support (career and psychosocial support), affective reaction (job satisfaction and organizational commitment), and the intent to participate in a formal mentoring program in an oilfield services organization. A 44-item electronic survey was utilized to collect data. The questionnaire was sent to 831 Field Engineers 1 (FE1's) in forty-two countries within an oilfield services organization. The sample included 341 respondents. Descriptive statistics, Cronbach's alpha estimates for reliability, factor analysis, correlation analysis, regression analysis, path analysis, and structural equation modeling were the analyses used in the study.

The researcher posited that cultural values amongst the FE1s do not differ significantly; moreover, the cultural values do not influence the FE1s perceived organization support. Further, it was found that FE1's perceived support (career and psychosocial) and affective reactions predicted the FE1's intent to participate in a formal mentoring program. 


\section{ACKNOWLEDGEMENTS}

The successful completion of my degree involved the support of my committee members and the love of my friends and family. First and foremost, I would like to thank Dr. Larry Dooley, my advisor and Mentor. It is because of Dr. Dooley that I began the Education and Human Resource Development program, and due to his constant care and attention, I have finally concluded my long and strenuous journey in obtaining this degree. The other important faculty members that have helped guide and mold this research were Dr. Toby Egan and Dr. Homer Tolson. Their willingness to give continuous and quality feedback far surpassed my expectations.

I would also like to thank Baker Hughes, International for allowing me to survey their Field Engineers 1. I would like to specifically thank Meta Rousseau and Gary Brown. Mr. Brown was instrumental in finding the appropriate group that fit my design needs and spent many hours with me perfecting the questionnaire for world-wide distribution. Mrs. Rousseau's care and guidance was critical in gaining access to Baker Hughes, the survey respondents, and most of all, in communication with the Baker Hughes team members. Her continuous support contributed greatly to the successful completion of my dissertation.

My family and my friends have been of great support during the past ten years - their love and affirmation has been immeasurable. I am grateful to my parents for their continued belief that this day would come. I am grateful to my friends for their ability to make me laugh and remember the joy. 


\section{TABLE OF CONTENTS}

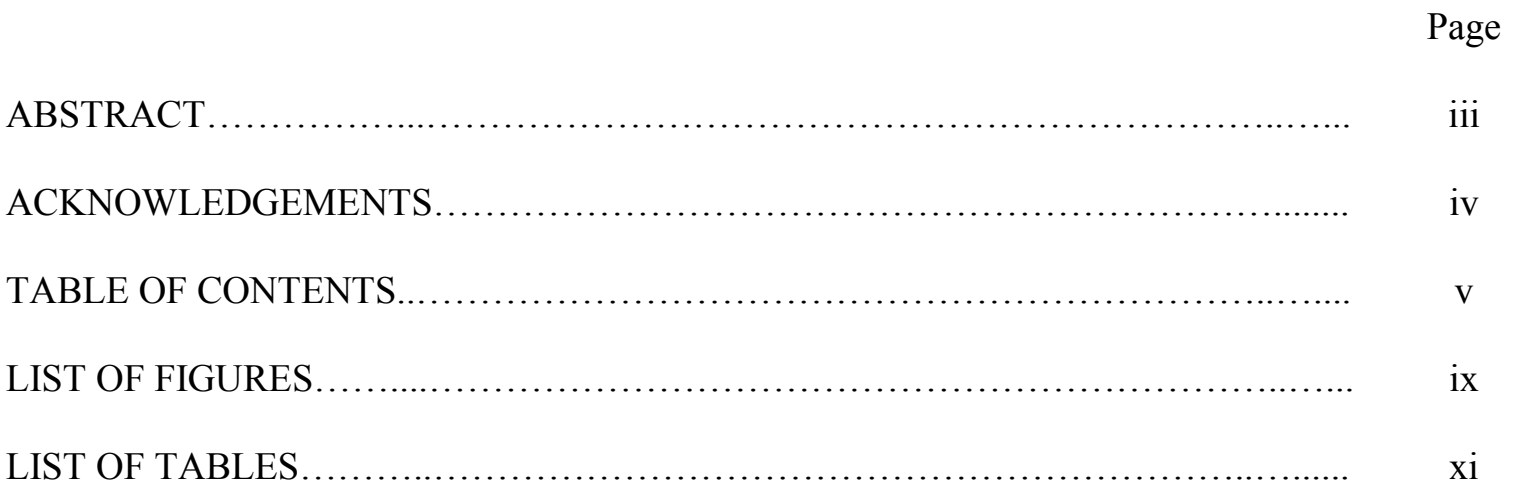

\section{CHAPTER}

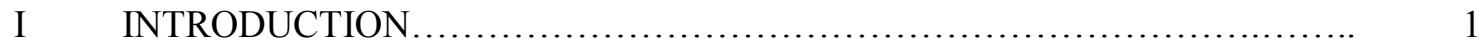

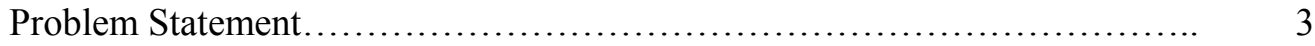

Purpose.............................................................. 6

Research Question and Research Hypotheses.............................. 6

Conceptual Framework............................................... 9

Definitions........................................................... 14

Assumptions and Delimitations........................................... 18

Significance of Study .................................................... 19

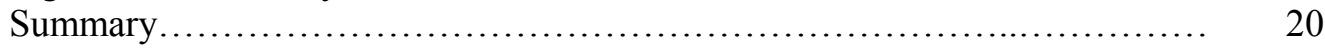

II REVIEW OF LITERATURE.......................................... 22

The Literature Review Process.......................................... 22

Theoretical Framework.................................................. 24

Cultural Values................................................. 25

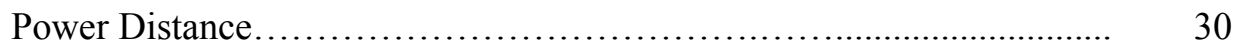

Uncertainty Avoidance............................................... 31

Individualism/Collectivism......................................... 32

Masculinity/Femininity.............................................. 34

Long-Term/Short-Term Orientation................................. 35

Country Level Comparisons..................................... $\quad 35$

Cultural Values [Diversity] and Mentoring......................... 37

Mentoring................................................................ 41

Definition of a Mentor.............................................. 44

Definition of a Mentoring Program................................ 46

Formal Mentoring..................................................... 48

Mentoring and Engineering ..................................... 50

LEAD Mentoring Program...................................... 52 
Mentoring Benefits: Perceived Organizational Support............... 53

Career Oriented Functions.......................................... 54

Psychosocial Functions................................................. 56

Affective Reaction: Job Satisfaction................................. 61

Job Satisfaction and Mentoring.................................... 61

Affective Reaction: Organizational Commitment...................... 64

Organizational Commitment and Mentoring........................ 68

Affective Reaction: Job Satisfaction and Organizational Commitment... 69

Summary

III METHODOLGY...

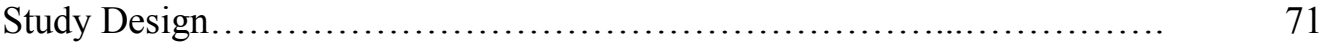

Population.......................................................... 72

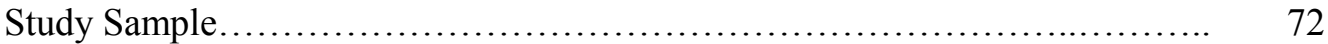

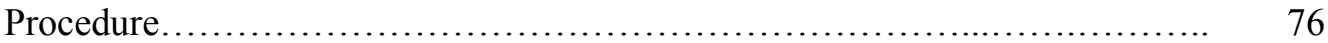

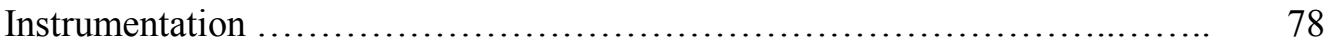

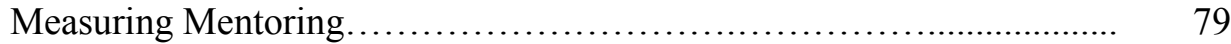

Measuring Intent to Participate in Mentoring........................ $\quad 80$

Measuring Job Satisfaction......................................... $\quad 80$

Measuring Organizational Commitment............................ 81

Measuring Cultural Values......................................... $\quad 81$

Data Analysis.................................................... 85

Descriptive Statistics........................................... 85

Reliability Analysis............................................ 86

Factor Analysis................................................. 86

Correlation Analysis............................................. 87

Regression Analysis............................................... 88

Simple Linear Regression....................................... $\quad 89$

Utility of Regression........................................... 90

Ordinal Regression............................................... 90

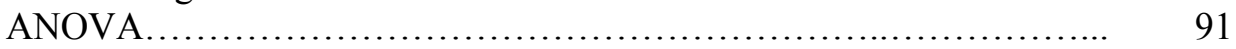

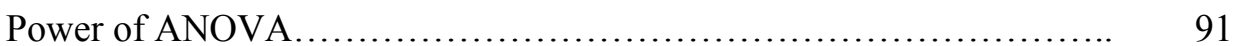

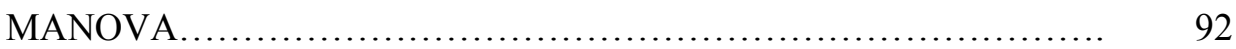

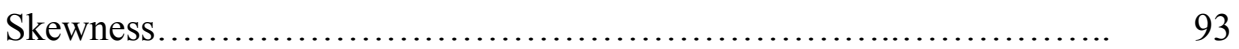

Welch Tests.................................................. 93

Path Analysis................................................... 94

Bartlett's Test............................................... 95

Kaiser-Meyer-Olkin Measure of Sampling Adequacy (KMO).......... 95

Chi Square.................................................... 95

Goodness of Fit................................................. 95

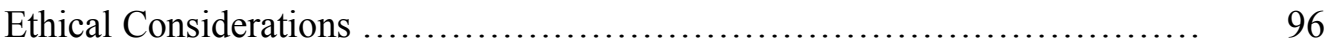

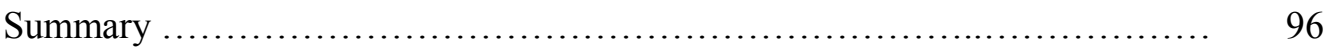

IV $\quad$ RESULTS AND FINDINGS ........................................... 97 
Descriptive Statistics ................................................. 99

Results of Factor Analysis .......................................... 102

Estimates of Reliability ............................................... 111

Results by Hypothesis ................................................ 113

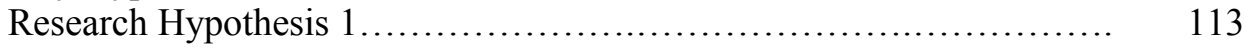

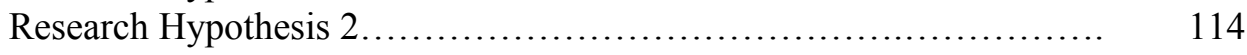

Breakdown by Region and Hemisphere - Research Hypothesis 2....... 117

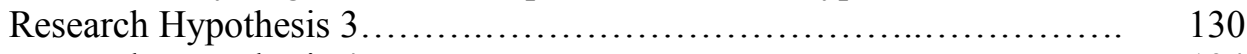

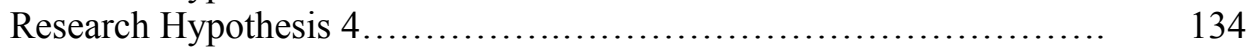

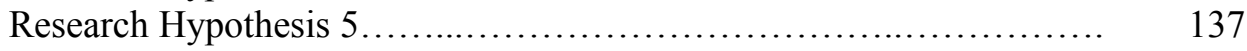

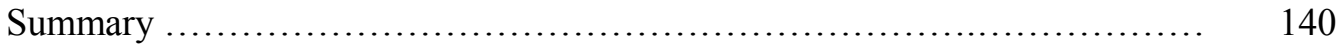

V DISCUSSION, CONCLUSION AND RECOMMENDATIONS.................. 141

Discussion........................................................ 141

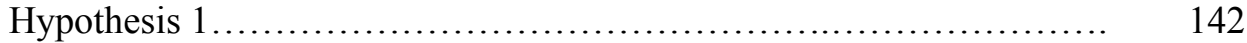

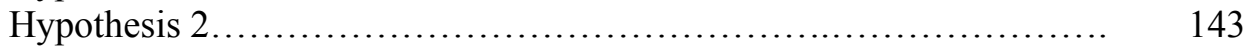

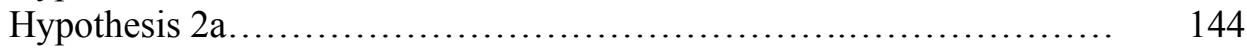

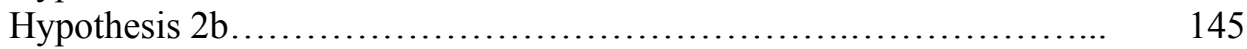

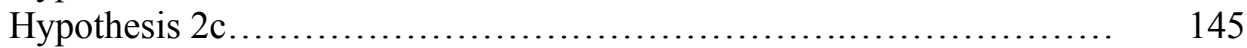

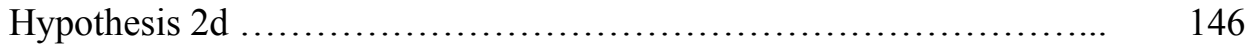

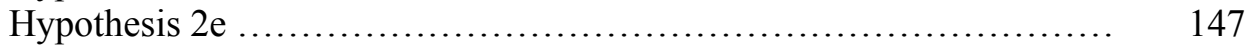

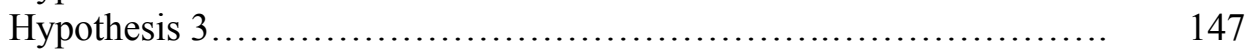

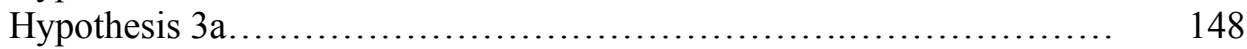

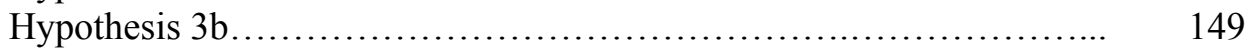

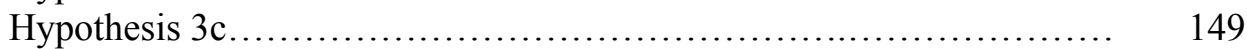

Hypothesis $3 d$................................................... 149

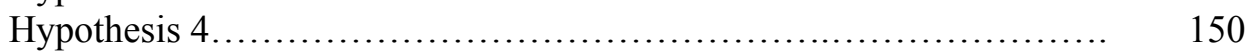

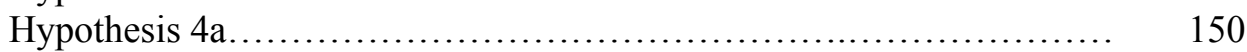

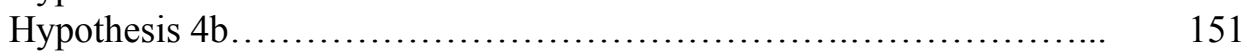

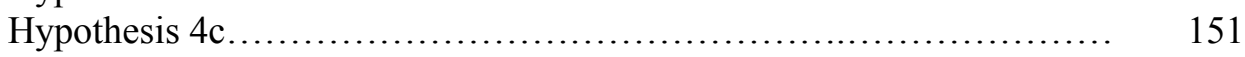

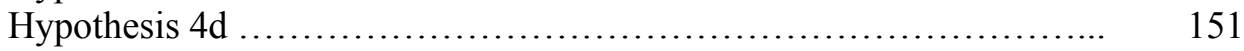

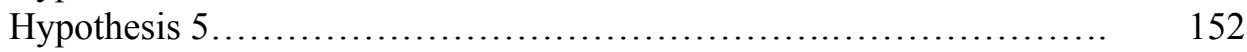

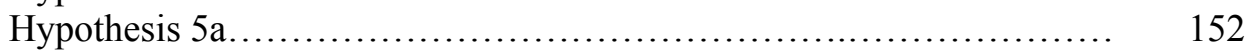

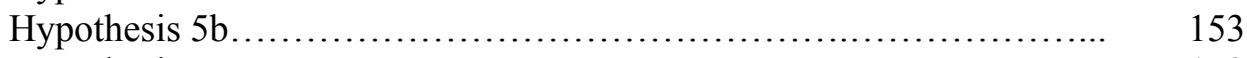

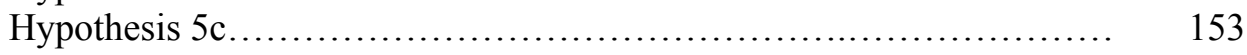

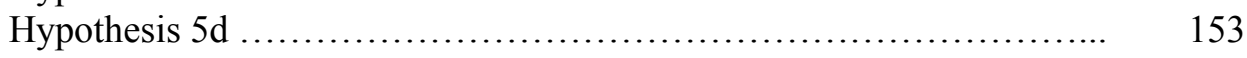

Models of the Study................................................ 154

Conclusions and Limitations........................................... 155

Implications for HRD Research and Practice ............................ 157

Recommendations and Directions for Future Research ................... 158

REFERENCES........................................................ 161

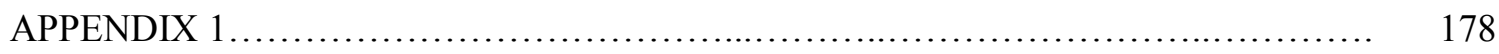




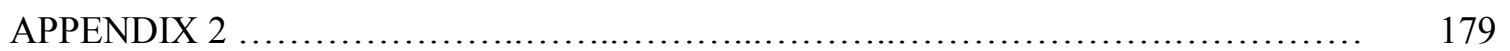

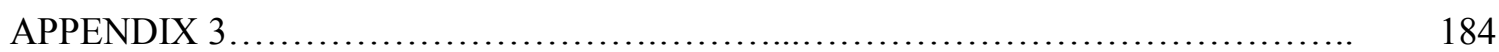

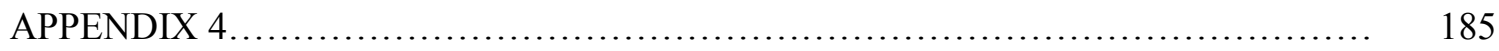

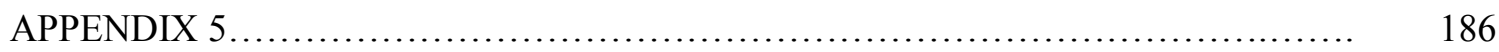

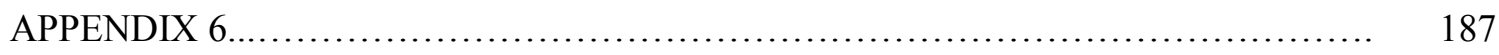

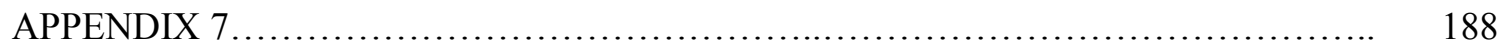

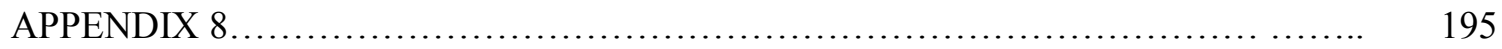

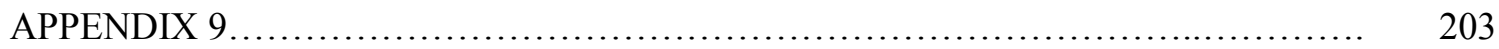

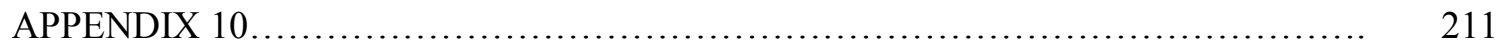

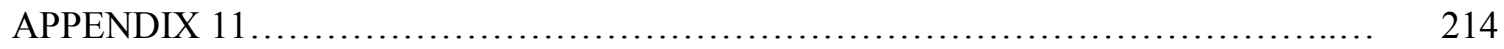

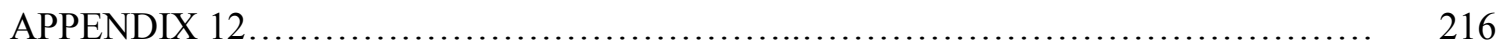

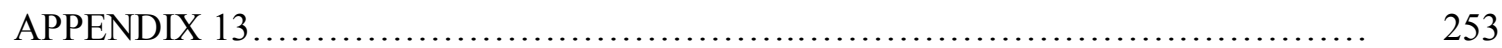

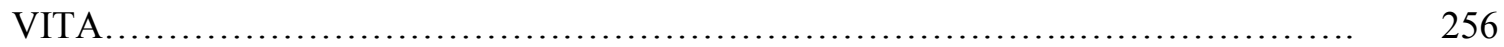




\section{LIST OF FIGURES}

FIGURE

Page

1 Conceptual Model of the Study......

12

2

Structural Model of Perceived Organizational Support and Intent to Participate in Mentoring.

114

3 Structural Model of Career Support and Cultural Values....

4 Structural Model of Perceived Organizational Support and Cultural Values Africa.

5 Structural Model of Perceived Organizational Support and Cultural Values Asia Pacific....

6 Structural Model of Perceived Organizational Support and Cultural Values Europe.

7 Structural Model of Perceived Organizational Support and Cultural Values Latin America.

8 Structural Model of Perceived Organizational Support and Cultural Values Middle East....

9 Structural Model of Perceived Organizational Support and Cultural Values North America....

10 Structural Model of Perceived Organizational Support and Cultural Values Russia \& Caspian.

126

11 Structural Model of Perceived Organizational Support and Cultural Values Eastern Hemisphere.

12 Structural Model of Perceived Organizational Support and Cultural Values Western Hemisphere.

13 Structural Model of Perceived Organizational Support and Intent to Participate in Mentoring.

14 Structural Model of Perceived Organizational Support and Intent to Participate in Mentoring..... 
15 Structural Model of Job Satisfaction and Organizational Commitment to Intent to Participate in Mentoring.

16 Structural Model of Perceived Organizational Support and Affective

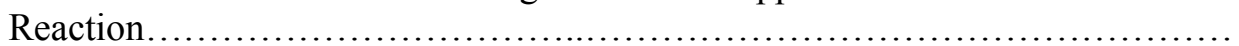

17 Structural Model of Job Satisfaction and Organizational Commitment to Intent to Participate in Mentoring.

18 Structural Model of Affective Reaction and Intent to Participate in Mentoring. 


\section{LIST OF TABLES}

TABLE

Page

$1 \quad$ Number of Respondents per Present Country of Citizenship................... 73

2 Summary of ANOVA for Cultural Indices across Years Employed................ 74

3 Summary of ANOVA for Cultural Indices across Years Employed in Current

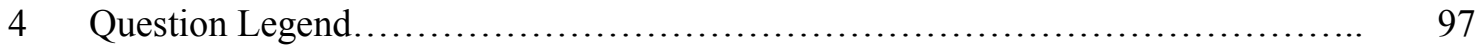

$5 \quad$ Descriptive Statistics................................................. 99

6 Summary of Sampling Adequacy for Factors.................................. 102

$7 \quad$ Total Variance Explained for Career Support................................ 103

8 Component Matrix for Career Support................................. 103

9 Total Variance Explained for Psychosocial Support.......................... 103

10 Component Matrix for Psychosocial Support................................ 104

11 Total Variance Explained for Perceived Organizational Support................... 104

12 Component Matrix for Perceived Organizational Support...................... 104

13 Total Variance Explained for Job Satisfaction............................. 105

14 Component Matrix for Job Satisfaction................................... 105

15 Total Variance Explained for Organizational Commitment...................... 105

16 Component Matrix for Organizational Commitment......................... 106 
TABLE $\quad$ Page

17 Total Variance Explained for Affective Reaction............................. 106

18 Component Matrix for Perceived Organizational Support....................... 107

19 Total Variance Explained for Cultural Values................................ 108

20 Component Matrix for Cultural Values...................................... 109

21 Reliability Estimates................................................. 111

22 Summary of ANOVA Significance for Hofstede's Indices....................... 113

23 Summary of Welch Significance for Hofstede's Indices......................... 114

24 Model Fit Indices of Structural Model of Perceived Organizational Support and 115

25 Summary Table for Perceived Organizational Support and Cultural Values.......... 115

26 Model Fit Indices of Structural Model of Career and Psychosocial Support and Cultural Values........................................................... 116

27 Summary Table for Career Support and Cultural Values......................... 117

28 Summary Table for Psychosocial Support and Cultural Values................... 117

29 Model Fit Indices of Structural Model of Perceived Organizational Support and Cultural Values - Africa..................................................

118

30 Summary Table for Perceived Organizational Support and Cultural Values Africa.....

119

31 Model Fit Indices of Structural Model of Perceived Organizational Support and Cultural Values - Asia Pacific 
TABLE $\quad$ Page

33 Model Fit Indices of Structural Model of Perceived Organizational Support and Cultural Values - Europe

34 Summary Table for Perceived Organizational Support and Cultural Values Europe.

Model Fit Indices of Structural Model of Perceived Organizational Support and Cultural Values - Latin America....

36 Summary Table for Perceived Organizational Support and Cultural Values - Latin America

37 Model Fit Indices of Structural Model of Perceived Organizational Support and Cultural Values - Middle East.

8 Summary Table for Perceived Organizational Support and Cultural Values - Middle East.....

39 Model Fit Indices of Structural Model of Perceived Organizational Support and Cultural Values - North America.

125

40 Summary Table for Perceived Organizational Support and Cultural Values - North America.

Model Fit Indices of Structural Model of Perceived Organizational Support and Cultural Values - Russia \& Caspian.

42 Summary Table for Perceived Organizational Support and Cultural Values - Russia \& Caspian.

43 Model Fit Indices of Structural Model of Perceived Organizational Support and Cultural Values - Eastern Hemisphere.

Eastern Hemisphere

45 Model Fit Indices of Structural Model of Perceived Organizational Support and Cultural Values - Western Hemisphere.

Summary Table for Perceived Organizational Support and Cultural Values Western Hemisphere

Model Fit Indices of Structural Model of Perceived Organizational Support and Intent to Participate in Mentoring. 
49 Model Fit Indices of Structural Model of Perceived Organizational Support and Intent to Participate in Mentoring............................................

50 Model Fit Indices of Structural Model of Job Satisfaction and Organizational Commitment and Intent to Participate in Mentoring.

51 Summary Table for Career Support and Psychosocial Support to Job Satisfaction and Organizational Commitment.

52 Model Fit Indices of Structural Model of Perceived Organizational Support and Affective Reaction.

55 Model Fit Indices of Structural Model of Affective Reaction and Intent to Participate in Mentoring. 


\section{CHAPTER I}

\section{INTRODUCTION}

Due to the globalization of business and the implications that globalization brings to management and organizations, Gerhart and Fang (2005) stated that organizations should increase attention to country differences. According to Blake-Beard, Murrell, and Thomas (2007), "Firms must address how to support and enable relationships among people who come from diverse cultures, backgrounds, and perspectives" (p.224). By acknowledging these differences and trying to understand the pretexts for their existence, managers and policymakers will be better able to integrate and/or redesign their entities' human resource management practices to achieve desired objectives and success on the global stage (Kelly, 2008). Hofstede, Neuijen, Ohayv and Sanders (1990) suggested that the four-dimensional model of national culture differences, as framed by Hofstede (1980), certainly does not epitomize the definitive truth about culture, but it has so far served as a useful framework for guiding research design in the field of national cultures.

Hofstede (1980) quantified culture and his proposal included a measurement for four dimensions of national culture: Individualism, Power Distance, Uncertainty Avoidance, and Masculinity. Hofstede and Bond (1988) added an "additional dimension independent of the original four, "Confucian dynamism" opposing a long-term to a short-term orientation in life and work" (Hofstede et.al, 1990, p. 288); this is referred to as Long-Term Orientation. Using Hofstede's dimensions, "Hofstede demonstrated that cultural variations between nations do exist and that they have the real potential to affect differences in HRM practices and perceptions" (Kelly, 2008, p.12). Hofstede posited that nationality is a formative factor in determining

This dissertation follows the style and format of Human Resource Development International. 
perceptions and this study's researcher believes these perceptions, based upon cultural values, can have an effect on an individuals' choice to participate, as well as their level of participation, in human resource development programs, like formal mentoring programs. It is estimated that more than one third of large organizations in the United States of America have a formal mentoring program (Bragg, 1989). However, as these organizations expand internationally, managers must be sensitive to the way individuals in different cultures are able to benefit from human resource development (HRD) programs such as formal mentoring programs (Budhwar \& Sparrow, 2002; Kelly, 2008).

Rosser (2004) stated, "The relationship between mentoring and HRD is observed through the three domains of HRD: organization development, training and development, and career development" (p. 48). Betts and Pepe (2006) stated, "Mentoring is a sustained relationship between a younger, less experienced individual (protégé) and an older, more experienced individual (mentor) dedicated to achieving long term success and fulfillment" (Burke, 1984; Kram, 1985; p. 105). Only within the last three decades, according to Ehrich, Hansford, and Tennent (2001), have formal mentoring programs become a part of human resource development strategies.

In support of Bragg (1989) and Rosser (2004), more than one third of large companies in the United States use mentoring to develop their employees, as organizations realize that mentoring advances the development of social capital (Lankau \& Scandura, 2007). Researchers suggest mentors help protégés in at least two domains - social (e.g., Thomas, 1993) and career (e.g., Fagenson, 1989; Kram, 1985). Kammeyer-Mueller and Judge (2008) theorized that "career functions include actions such as providing the protégés with human capital enhancement opportunities and links to powerful individuals in the organization, [and] psychosocial functions 
include counseling the protégé about anxieties and uncertainty, providing friendship and acceptance, and role modeling” (p. 270).

According to Egan and Song (2008), "Formal mentoring may bring significant benefits to participants: protégés of the mentoring program cannot only benefit psychologically, including increased job satisfaction, higher commitment to their organization and perceived better fit with their organization, but also demonstrate higher job performance immediately after the program" (p. 358-359). Likewise, positive outcomes for the participants also create positive outcomes for the organization. According to Ehrich, Hansford and Tennent (2001), "Positive outcomes for the organization in business settings included improved productivity, retention of staff, promotion of loyalty, an improved workplace, and that mentoring facilitates change" ( $\mathrm{p}$. 12).

Allen, Eby, Poteet, Lentz, and Lima, (2004) found a significant positive relationship between mentoring and job satisfaction, such that mentoring is the likely driver of employee outcomes. Furthermore, Egan and Song (2008) concluded "that the significant positive effects of mentoring on protégés job satisfaction and organizational commitment is likely a characteristic outcome of formal mentoring" (p. 358).

\section{Problem Statement}

In the past few decades, progress has been made by researchers with regard to the benefits of HRD, namely development programs such as formal mentoring. In her seminal work, Kram (1985) detailed mentoring functions to include career and psychosocial; moreover, Kram (1985) explained how mentoring relationships, in terms of career and psychosocial functions, benefit an organization by adding the additional benefits of job satisfaction and organizational commitment. According to Raabe and Beehr (2003), positive outcomes for employees involved 
in formal mentoring programs include career self-reliance, enhanced career development, and individual career growth; whereas positive outcomes for the organization "included retention of valued employees, increased productivity, and acceleration in employee development" (p. 277).

According to Ensher and Murphy (1997), "Benefits that are said to accrue to the organization include: (a) the integration or socialization of individuals into the operating norms and informal power structure; (b) increased organizational communication as mentors and protégé's form alliances across levels and departments; (c) management development and succession planning information; and (d) increased productivity and decreased turnover" (Wilson \& Elman, 1990; Zey, 1984; p. 461). "It is important to mention the findings of Hegstad and Wentling (2004) and Egan and Rosser (2004), who concluded that companies enjoy improved performance, increased motivation, higher job satisfaction, and increased organizational commitment as benefits of mentoring relationships" (Rosser, 2004, p.50). By understanding the foundational outcomes - perceived support - a strong basis is set for the importance of formal mentoring in developing employees.

Multinational companies introducing mentoring programs around the world have attempted a variety of strategies (Mezias \& Scandura, 2005). The major issue that seems to plague multinational mentoring programs is mentor/protégé mismatch. Researchers have shown that culture impacts formal mentoring relationships; however, regarding race and culture dynamics, there is an absence in the mentoring literature, as most of the researcher's highly regarded models, theories, and empirical studies either exclude race as a factor or include samples that lack diversity such that race [culture] is relegated to 'unexplained variance' (BlakeBeard, Murrell, \& Thomas, 2007). Unexplained variance, according to Blake-Beard, Murrell, and Thomas (2007), is "one of the areas that provides both a primary opportunity for research 
and a significant challenge to the advancement of knowledge" concerning how mentoring is defined within the context of race [culture]" (p. 233).

Noe, Greenberger, and Wang (2002) suggested that "most of the research on diversified mentoring relationships has considered diversity from the perspective of age, gender, race, and nationality" (p.147). Ragins (1997) defined diversified (diverse) mentoring relationships as “...comprising mentors and protégés who differ on the basis of race, ethnicity, gender, sexual orientation, class, religion, disability, or other group memberships associated with power in organizations" (p. 24). With globalization of corporations, diverse mentoring relationships are more commonplace; however, research has mainly focused on race differences. In fact, Ragins (1997) suggested that "research on cross-race relationships reveals distinct and important differences in mentoring processes and outcomes" (Atkinson, Neville, \& Casas, 1991; Burke, McKeen, \& Mckenna, 1993; Thomas, 1990, 1993, p. 482). Little research exists regarding the types of benefits and barriers that mentors and protégés experience in mentoring relationships that are cross-cultural.

In his seminal work, Hofstede (1980) detailed the different cultural factors affecting organizations and their HR practices. Ragins (1997) believed that "a link between diversity [culture] and mentorship can also help expand and define the newly emerging field of mentorship in organizations" (p. 483). HRD practitioners need to understand where and how formal mentoring should be utilized in developing individuals in order to improve organizations.

Although a substantial amount of research has been conducted in the last decade regarding mentoring, the research pertaining to formal mentoring programs, cultural values, perceived organizational support, and the influence of formal mentoring programs on affective reactions (employee job satisfaction and employee organizational commitment), in the oilfield services industry does not exist. Several articles have been published regarding formal 
mentoring, perceived organizational support, and the influence of formal mentoring programs on affective reactions and these publications were used in the literature review as guiding works for this study.

\section{Purpose}

The researcher, through an exploratory model testing with an oilfield services organization, developed a model that can be used to predict an employee's intent to participate in a formal mentoring program. The researcher sought to uncover the extent to which cultural values in an oilfield services corporation differ among employees. The researcher also explored cultural values on organizational support as defined by Kram (1985) - career and psychosocial support - within the oilfield services industry, specifically within the cultural contexts as framed by Hofstede (1980). The researcher sought to uncover whether an employee's cultural values influences their intent to participate in a formal mentoring program based upon the employee's perceived support (career and psychosocial). The researcher also sought to uncover employees' intent to participate in a formal mentoring program based on the employees' affective reactions (job satisfaction and organization commitment).

\section{Research Question and Research Hypotheses}

Utilizing data from the proposed survey research of employees in a multinational oilfield services company, the researcher examined the following:

Employee cultural values and perceptions of organizational support on affective reactions and the desire [interest/non interest] to participate in a companysponsored formal mentoring program.

The above research question was explored using the following research hypotheses: 
Research Objective 1: To determine if cultural values, as framed by Hofstede's (Employee Values Survey, 1994), differ among employees of an oilfield services corporation.

- Research Hypothesis 1: Employees' cultural values will significantly differ among employees in an oilfield services corporation - across Hofstede's (1980) five cultural dimensions.

Research Objective 2: To examine the relationships between cultural values and perceived organizational support.

- Research Hypothesis 2: Employees' individual cultural values - as framed by Hofstede (1980) - will be unrelated to their perceptions of support from their organization.

- Hypothesis 2a: There will be no significant relationship between employee individualism and their perception of support from their organization.

○ Hypothesis $2 \mathrm{~b}$ : There will be no significant relationship between employee Power Distance and their perception of support from their organization.

- Hypothesis 2c: There will be no significant relationship between employee uncertainty avoidance and their perception of support from their organization.

- Hypothesis 2d: There will be no significant relationship between employee masculinity and their perception of support from their organization.

- Hypothesis 2e: There will be no significant relationship between employee long term orientation and their perception of support from their organization. 
Research Objective 3: To determine the extent to which perceived support can be used to predict oilfield services company employee interest/non interest in formal mentoring program participation.

- Research Hypothesis 3: Employee perceived support can be used to accurately predict interest/ non interest in formal mentoring program participation.

$\circ$ Hypothesis 3a: There will be a significant negative relationship between employee perceived career support and interest in formal mentoring program participation.

○ Hypothesis $3 \mathrm{~b}$ : There will be a significant negative relationship between employee perceived psychosocial support and interest in formal mentoring program participation.

- Hypothesis 3c: Employees with high levels of perceived career support will show no interest in formal mentoring program participation.

- Hypothesis 3d: Employees with high levels of perceived psychosocial support will show no interest in formal mentoring program participation.

Research Objective 4: To determine if perceived support influences oilfield services employees' affective reactions.

- Research Hypothesis 4: Employee perceived support is positively related to affective reactions.

- Hypothesis 4a: There will be a significant positive relationship between employee perceived career support and employee job satisfaction.

- Hypothesis 4b: There will be a significant positive relationship between employee perceived psychosocial support and employee job satisfaction. 
- Hypothesis 4c: There will be a significant positive relationship between employee perceived career support and employee organizational commitment.

- Hypothesis 4d: There will be a significant positive relationship between employee perceived psychosocial support and employee organizational commitment.

Research Objective 5: To determine if affective reactions can be used to accurately predict oilfield services employee interest in formal mentoring program participation.

- Research Hypothesis 5: Affective reactions of oilfield services employees can be used to accurately predict participation in a formal mentoring program.

○ Hypothesis 5a: There will be a significant positive relationship between employee job satisfaction and interest in formal mentoring program participation.

○ Hypothesis $5 \mathrm{~b}$ : There will be a significant positive relationship between employee organizational commitment and interest in formal mentoring program participation.

- Hypothesis 5c: Employees with high levels of job satisfaction will show no interest in formal mentoring program participation.

Hypothesis 5d: Employees with high levels of organizational commitment will show no interest in formal mentoring program participation.

\section{Conceptual Framework}

Some of the most commonly cited theories relating to mentoring, in literature reviewed for this study, were human capital theory, exchange theory and developmental theory. The 
economic idea encompassing human knowledge and skills as capital was first formalized into theory by Theodore Schultz. In his article Investment in Human Capital, Schultz (1961) argued that both knowledge and skill are a form of capital, and that this capital is a product of conscious investment. Hayes, Alagaraj and Dooley (2003) depicted three key relationships in human capital theory: "First, an investment in training leads to increased learning. Second, as learning increases, so does production. And third, as productivity increases, both individuals and the organization realize an increase in earnings" ( $₫ 15)$. Human capital theory concentrates on education and training and as Kagan (2000) posited "society needs to invest in people for the sake of a stronger, more productive economy, and also to increase the opportunities and choices open to the individual" ( 8 ). "Common to human capital theory and the other theories categorized under economic theory was the notion that mentoring was an exchange which yielded some type of reciprocity for the mentor and mentee" (Ehrich, Hansford \& Tennent, 2001, p. 6).

According to Raabe and Beehr (2003), "much of the theoretical base for the mentoring relationship relies on social exchange theory (Blau, 1964; Homans, 1958; Thibaut \& Kelley, 1959). In fact, a social exchange perspective highlights the symbiotic connection in the mentor/mentee relationship and focuses on the potential benefits for both mentees and mentors. According to Rosser (2004), social exchange theory is the most acknowledged theoretical foundation in mentoring relationships (Hegstad \& Wentling, 2004; Ragins \& Scandura, 1994). Social exchange theory originated in economics and is applied in a social milieu to relationships. Moreover, with regard to social exchange theory, the criterion variables most frequently chosen are "turnover intentions, organizational commitment, and job satisfaction, because they fit with the social exchange theme" (Raabe \& Beehr, 2003, p. 273). 
In order to elucidate why certain individuals receive mentoring and others do not, researchers utilized selection theories. Ehrich, Hansford and, Tennent (2001) suggested lack of sponsorship or tokenism (following Kanter, 1977) and pipeline theory (Fullbright, 1985). Moreover, other theories used are attribution theory, feminist theory and sociological theory. The researchers that used these theories expressed mentoring relationships in terms of race, gender and socio-economic status. Ehrich, Hansford, and Tennent (2001) concluded that "these theories maintain that some individuals are more likely to be mentored than others because of their attributes (such as gender, race, similarity to the mentor, or socio-economic status)" (p. 7).

With regard to culture, Emery and Oertel (2006) indicated that "motivational theories are closely tied to the values, beliefs, and norms of the society, i.e. a society's culture (Gagne \& Deci, 2005)" (p.7). In fact, Emery and Oertel (2006) posited that " Victor Vroom suggested with his Expectancy Theory that a person's motivation is based on the product of his or her valence (the value of an individual goal), expectancy (probability of successfully accomplishing a task), and instrumentality (probability that the successful accomplishment of the task will result in achieving a desired goal)" (p. 7). Using Expectancy Theory, Vroom (1964) postulated that individuals are motivated based on perceptions and value systems so they perform activities that produce the highest probability of desired outcomes (Hofstede, 1994). Emery and Oertel (2006) suggested that "since a person's perception is influenced by one's value system, it seems reasonable to believe that culture-based values might moderate the values of an individual's expectancies, instrumentalities and valences and therefore have an effect on the individual's motivation" (p. 8).

In regard to culture, Hofstede's (1980) five-dimensional model of national culture differences has served as a useful framework for guiding research design in the field of national cultures. Hofstede (1980) assumed that the values of individuals within a nation are 
(substantially) more similar than the values of individuals from different nations. Yang, Wang and Drewry (2009) suggested using Hofstede's framework of culture because "Hofstede's dimensions are one of the widely accepted and well-studied sets of cultural dimensions" (p. 325). Hofstede (1980) proposed and created measures of four dimensions of national culture: Individualism, Power Distance, Uncertainty Avoidance, and Masculinity; and in 1988, Hofstede and Bond added an additional dimension: Long-Term Orientation. Using Hofstede's dimensions, "Hofstede demonstrated that cultural variations between nations do exist and that they have the real potential to affect differences in HRM practices and perceptions" (Kelly, 2008, p. 12).

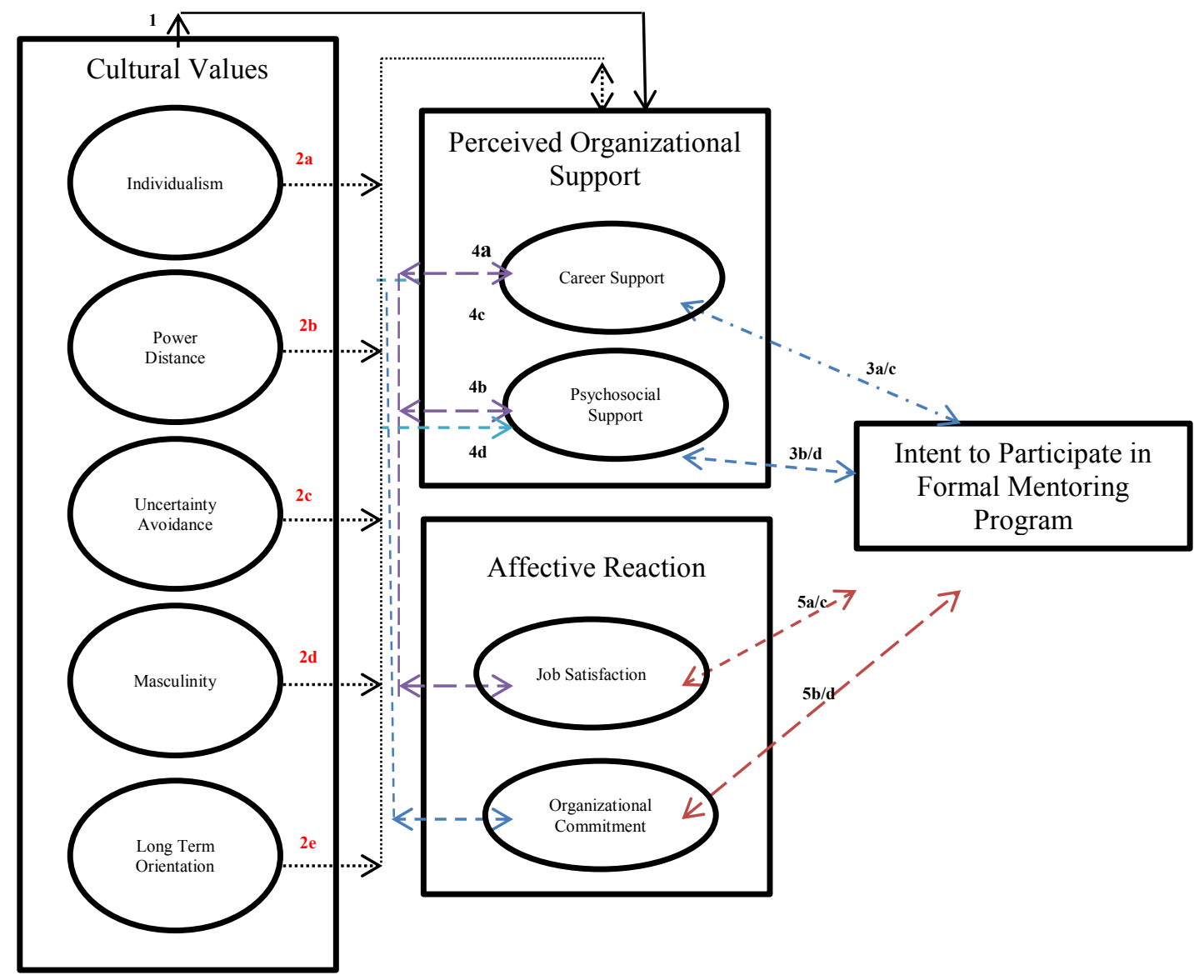

Figure 1 Conceptual Model of the Study 
Hypothesis Legend

1 Employees' cultural values will significantly differ among employees in an oilfield services corporation - across Hofstede's (1980) five cultural dimensions.

2 Employees' individual cultural values - as framed by Hofstede (1980) - will be unrelated to their perceptions of support from their organization.

2a There will be no significant relationship between employee individualism and their perception of support from their organization.

$2 \mathrm{~b}$ There will be no significant relationship between employee Power Distance and their perception of support from their organization.

2c There will be no significant relationship between employee uncertainty avoidance and their perception of support from their organization.

$2 \mathrm{~d}$ There will be no significant relationship between employee masculinity and their perception of support from their organization.

$2 \mathrm{e}$ There will be no significant relationship between employee long term orientation and their perception of support from their organization.

3 Employee perceived support can be used to accurately predict interest/ non interest in formal mentoring program participation.

3a There will be a significant negative relationship between employee perceived career support and interest in formal mentoring program participation.

$3 \mathrm{~b}$ There will be a significant negative relationship between employee perceived psychosocial support and interest in formal mentoring program participation.

$3 c$ Employees with high levels of perceived career support will show no interest in formal mentoring program participation.

3d Employees with high levels of perceived psychosocial support will show no interest in formal mentoring program participation.

4 Employee perceived support is positively related to affective reactions.

4a There will be a significant positive relationship between employee perceived career support and employee job satisfaction.

$4 \mathrm{~b}$ There will be a significant positive relationship between employee perceived psychosocial support and employee job satisfaction.

$4 \mathrm{c}$ There will be a significant positive relationship between employee perceived career support and employee organizational commitment.

4d There will be a significant positive relationship between employee perceived psychosocial support and employee organizational commitment.

5 Affective reactions of oilfield services employees can be used to accurately predict participation in a formal mentoring program.

5a There will be a significant positive relationship between employee job satisfaction and interest in formal mentoring program participation.

$5 b$ There will be a significant positive relationship between employee organizational commitment and interest in formal mentoring program participation.

$5 \mathrm{c}$ Employees with high levels of job satisfaction will show no interest in formal mentoring program participation.

5d Employees with high levels of organizational commitment will show no interest in formal mentoring program participation. 


\section{Definitions}

Terms of this study will be better understood within the parameters of their intended definitions.

LEAD Program: The LEAD (Learn, Excel, Achieve, Develop) Field Engineer Development Program includes in-depth training programs focused on industry and enterprise knowledge, warehouse and field installation processes, and basic product line knowledge. The initial focus of the LEAD program is on Field Operations, starting with Career Development Programs for FEIs. The LEAD program is an employee development program that enables career progression through a sequence of competency-based development steps. LEAD involves both theoretical and hands-on learning; it is flexible and adaptable to different job requirements and work environments. In addition, the LEAD program assigns a mentor, who works closely with the FEIs, their managers and training personnel to ensure a comprehensive, learning experience. The mentor for a FEI supports and directs the FEIs through the program curriculum, assisting in preparing for yearly assessments and final qualification and, ultimately, enable mentees to become mentors to less experienced engineers.

Career Development: An all-encompassing term that allows a partnership to form between the individual and the organization such that the organization provides the resources for career development, the supervisor provides the counseling and coaching and the employee provides the direction and motivation (Knowdell, 1996).

Career Support: Kram (1985) stated "career functions are those aspects of the relationship that enhance learning the ropes and preparing for advancement in an organization” (p.22). According to Ehrich, Hansford, and Tennent (2001) and Ozkalp, Kirel, Sungur, and Ozdemir (2008), "the career functions of mentoring focus on the 'external' career progress attributes such as sponsorship, coaching, protection, visibility and exposure" (p.6). 
Culture: Yang, Wang and Drewry (2009) defined culture as "a collectively shared social knowledge and understanding that is reflected in values, beliefs and habitual social norms within a group of individuals" (p. 325).

Ethnicity: According to Anthias and Davis (1992), ethnicity factors are 'this origin, mythical or 'real', [and] can be historically, territorially, culturally, or physiognomically based' (p. 423). Kamenou (2007) stated that ethnicity embraces a number of factors, including "cultural and racial characteristics and it is viewed here as broader than 'race' encompassing cultural diversity, values and traditions" (p. 1999).

Field Engineer I (FEI): Field Engineer I job details are as follows: University Degree in Engineering or Applied Science degree and general knowledge of basic engineering principles and good mechanical aptitude. FEI's have the ability to work and communicate well with others. Under direct supervision, a FEI receives and follows specific detailed instructions on all work performed in the field where work is closely managed by more experienced Field Engineers. FEI learns to run the production line's tool(s) and/or services as assigned. FEIs must be able to perform basic maintenance on various types of field equipment and to determine the equipment's current operational status at the well site. A FEI performs all required responsibilities safely and as described in work instructions, job-site analyses and training manuals. FEIs execute other related duties as assigned and conduct all business activities in accordance with the organization's policies, requirements and core values. All FEIs complete required training through participation in Phase One of the LEAD Field Engineer Development Program which includes in-depth training programs focused on industry and enterprise knowledge, warehouse and field installation processes, and basic product line knowledge.

Formal Mentoring Program: According to the Mentoring Program Handbook (2003), "A formal mentoring program is a system for encouraging more widespread development of these 
relationships. A formal mentoring program offers a structured approach to developing employee talents and abilities. A formal mentoring process capitalizes on the experiences of successful individuals (mentors) in the organization who are committed to helping develop a highly skilled, high-performing workforce." Rosser (2004) defined formal mentoring programs as relationships developed by a third party which usually "have measurable productivity goals and a set time frame in which to accomplish the goals" (p. 34).

Formal Mentoring 1: Respondents who chose 'Yes', implying that the employee is currently participating in a mentoring relationship through the LEAD program and that they would like to be assigned a mentor (Training Engineer).

Formal Mentoring 2: Respondents who chose 'No', implying that the employee was not currently participating in a mentoring relationship through the LEAD program and that they would like to be assigned a mentor (Training Engineer).

Human Resource Development: The HRD faculty at Texas A\&M University defined human resource development as "the process of improving learning and performance in individual, group, and organization contexts through domains of expertise such as lifelong learning, career development, training and development, and organization development" (Texas A\&M University, 2002)."

Job Satisfaction: The presence of a certain level of contentment inherently felt through employment. Egan, Bartlett and Yang (2004) defined job satisfaction "as an employee's affective reactions to a job based on comparing desired outcomes with actual outcomes" (Cranny, Smith, \& Stone, 1992; p. 283). Job satisfaction is a multifaceted construct that reflects the expectations of satisfaction in employment (Porter \& Steers, 1973).

Mentor: Ragins (1997) defined mentors "as individuals with advanced experience and knowledge who are committed to providing upward mobility and support to their protégés' 
careers" (Hunt \& Michael, 1983; Kram, 1985; p. 484). According to Donaldson, Ensher, and Grant-Vallone (2000), a mentor is defined as "a more experienced employee who advises, counsels, or otherwise enhances the personal development of a less experienced employee" ( $p$. 238). Bass (1990) defined a mentor as "a trusted counselor who accepts a guiding role in the development of a younger or less-experienced member of the organization” (p. 90).

Mentoring: Betts and Pepe (2006) defined mentoring as "a sustained relationship between a younger, less experienced individual (protégé) and an older, more experienced individual (mentor) dedicated to achieving long term success and fulfillment" (Burke, 1984; Kram, 1985; p. 105). Kram (1985) defined mentoring as "an experienced, productive manager who relates well to a less-experienced employee and facilitates his or her personal development for the benefit of the individual as well as that of the organization" (p. 65).

Organizational Commitment: Refers to an emotional state that is strong enough to direct an employee into making decisions solely concerning the organization rather than themselves. Mowday, Steers, and Porter (1979) defined organizational commitment as identification and involvement with the organization focused on credence in the organization's values and goals, which leads to the exerting effort on behalf of the organization, and a desire to continue employment with the organization.

Psychosocial Support: Kram (1985) stated "psychosocial functions are those aspects of a relationship that enhance a sense of competence, clarity of identity, and effectiveness in a professional role." (p.22). According to Ehrich, Hansford, and Tennent (2001) and Ozkalp, Kirel, Sungur, and Ozdemir (2008), "psychosocial development is more inclined to be 'inner oriented' and includes counseling, role modeling, friendship, and acceptance and confirmation" (p.6). 
Traditional Mentoring Program: Turban and Lee (2007) defined "a traditional mentoring relationship as an intense interpersonal exchange between a senior, experienced, and knowledgeable employee (i.e., the mentor) who provides advice, counsel, feedback, and support related to career and personal development for a less experienced employee (the protégé)" (Noe, Greenberger \& Wang, 2002; p. 21; Kram, 1985).

Turnover Intentions: Includes positive and negative intentions by the employee regarding the employee's perception of the length of their employment. According to Egan, Yang, and Bartlett (2004), "turnover intention is defined as a conscious and deliberate willingness to leave the organization" (p. 286).

Values: Values are an attribute of individuals as well as of collectivities; culture presupposes a collectivity. Hofstede (1980) defined value as a "broad tendency to prefer certain states of affairs over others" (p. 19).

\section{Assumptions and Delimitations}

The following is a discussion of the assumptions for this study as well as a discussion of the strengths and weaknesses of this study. It was assumed that the potential respondents were capable of understanding and responding authentically to the survey questions.

First of all, the research data collected was limited to collection of data within one corporation in the oilfield services industry. As understood by the agreement between the organization and the researcher, the findings of this study are confidential as sharing results across multiple firms created cause concern. However, one benefit to using a single oil corporation is that the corporation has international holdings. These holdings vary in target market classification, employee demographics, and organizational size. Furthermore, greater consistency in the study was realized by using only one oilfield services corporation. 
A second limitation was that the employees involved in the study were determined by human resource management within the specified regions. The researcher was allowed to select all participants from amongst Field One Engineers (FE1's).

Finally, this study was limited to the examination of attributes as uncovered through the literature review: cultural differences, formal mentoring, career and psychosocial development, career benefits, growth opportunities, perceived organizational support, organizational commitment, job satisfaction, and turnover intentions.

\section{Significance of the Study}

In an effort to maintain a competitive advantage, companies increase the scope of their operations, seeking unexploited markets and fresh labor pools. However, together with the advantages added by the utilization of new labor forces, companies must learn how to conduct business in new regions and within different cultures. Hofstede (1983) established that cultural variations affect the international human resource development practices within organizations as well as employee perceptions of these practices. Because of increasing business pressures and the interconnectedness of the global market, researchers are trying to determine how well human resource development practices such as formal mentoring programs, can be transposed across different cultures and to identify practices that should be modified (Kelly, 2008).

According to McLean and McLean (2001), the intended audiences and benefactors of human resource development are individuals, organizations, communities and even nations. Employers clearly recognize the need for human resource development; employee commitment can reduce job turnover and increase productivity, which in turn, increases the competitiveness of an organization. Organizations instituting human resource development programs, such as formal mentoring, reinforce employee commitment through perceived organizational support 
and the development of meaningful and valued relationships. "Mentoring is one of the oldest forms of human development" (Shea, 1994, p. 13), and it is agreed that "mentoring is the most intense and powerful one-on-one developmental relationship" (Wanberg, Welsh, \& Hezlett, 2003, p. 5). As such, organizations are increasing their use of mentoring as an employee development tool (Noe, Greenberger, \& Wang, 2002; Rosser, 2004).

Allen, Shore and Griffeth (2003) suggested that supportive human resource development programs are related directly to employee satisfaction and withdrawal. Further research findings link "human resource practices to commitment, satisfaction, turnover intentions, and turnover" (Allen, et al., 2003, p. 106). By matching human resource development practices with cultural contexts, organizations expect to preserve the advantages gained by globalization. Despite the growing interest in cultural diversity and human resource development practices, the researcher did not uncover any published research that examined the cultural perceptions and differences in values within the oil industry. The researcher is using this study to decipher the association between employee perceptions and human resource development programs within different cultures in the oilfield services industry. Furthermore, the researcher will add to the value survey model, as developed by Hofstede in 1994, to further human resource development and management techniques as related to oilfield services organizations.

\section{Summary}

In Chapter 1, the researcher provided an introduction to the topic of the study and a brief discussion about the objectives of the study. Then, a description of the problem was provided and arguments supporting the need for the study were made. Next, the purpose of the study and the research hypotheses were presented. Then, the theoretical framework and the conceptual model of the study were presented; followed by the definition of the terms used in the study, and 
the assumptions and limitations of the study design. Finally, the significance of the study was provided. In Chapter 2, that follows, the study's theoretical framework will be presented and a review of literature on the four main objectives involved in the study will be discussed. 


\section{CHAPTER II}

\section{REVIEW OF LITERATURE}

This review of literature begins with background elements related to the methods of the study. This chapter will introduce and identify culture and describe the varieties and cultural differences using Hofstede's theoretical framework. The chapter then describes mentoring, defines mentoring, describes the types and functions of mentoring relationships, and lastly, will detail the benefits received from mentoring.

\section{The Literature Review Process}

The literature review process involved a search and selection of articles followed by a summarization and synthesis of the selected articles. The following criteria were used to select the articles for the variables: cultural values, organizational support (career and psychosocial), and affective reaction (job satisfaction and organizational commitment). These selection criteria limitations did not apply in the selection of articles for the literature review in general or for the other chapters of the dissertation.

- Journal articles related to the constructs cultural values (individualism, power distance, uncertainty avoidance, masculinity, and long term orientation), organizational support (career and psychosocial), and affective reaction (job satisfaction and organizational commitment).

- The initial search for articles was limited to ten of the top journals in Human Resource Development and related fields as determined by the researcher through informal inquiry. 
- The search period was ten years, from year 2000 to March 2010. Frequently occurring citations or seminal works from identified articles which superseded the search period were also included. As identified below, there were many articles, dating back to the 1960 s and earlier, that were determined to be seminal works associated with variables under investigation in this study reported herein.

- The ten journals selected were Human Relations, Journal of Management, Journal of Applied Psychology,Training and Development Journal, The International Journal of Human Resource Management, Academy of Management Journal, Human Resource Development International, The International Journal of Human Resource Management, Journal of Vocational Behavior, and Human Resource Development Quarterly. These journals are from fields related to the topic of study, human resource development, organizational psychology, business and management, and organizational behavior. As noted below, articles from several other journals were included.

The following criteria were used in the selection of central or seminal works contributing to the theroetical framework of the study, spefically relating to the variables:

- Studies most commonly cited in the selected articles.

- Direct relevance of the article to the study's theoretical framework and to the variables in the study.

- Significance of an article's theoretical and/or empirical contribution.

Besides these journals, selected books, such as Mentoring at Work: Developmental Relationships in Organizational Life by Kram (1988), International Dimensions of Organizational Behavior by Adler (2002), The Handbook of Mentoring at Work: Theory, Research, and Practice by Ragins 
and Kram (2007), Culture's Consequences: International Differences in Work-Related Values by Hofstede (1980), and Cultures Consequences: Comparing values, behaviors, institutions, and organizations across nations by Hofstede (2001) were identified as seminal works.

A Texas A\&M University library search engine was used to search for articles and the ERIC-EBSCO database provided access to all the journal articles. A year-by-year, volume-byvolume, issue-by-issue search was conducted for each variable. The identified articles were downloaded and either stored in a separate electronic file or printed and the hard copy was organized in binders.

The summarization of articles pertaining to the variables was started with the most recent articles for earch variable, including the seminal articles for that variable and proceeded to the oldest articles. The over 170 page summary table included the year of the study, the authors, and a summary of key points and study outcomes (due to page length restrictions, the full summary table is not included in this document). Only key aspects of the article and articles relevant to the topic of this study are included in the summary. The main purpose of the summary table was to build an initial "literature data bank" for the variables in order to provide a point of reference. In total, 81 articles from fifteen journals are included in the summary tables. This did not include other references used for the literature review, articles reviewed after March 2010, and book chapters. The literature review integrates information from the summary of articles.

\section{Theoretical Framework}

This study is based on the theoretical assumption that factors, cultural values specifically, influence an individual's work attitude and work behavior. Specifically, the study's theoretical framework is based on theories and models that suggest a relationship between 
cultural values and perceived career and psychosocial support (organizational support/work attitude), job satisfaction and organizational commitment (affective reaction/work attitude) and intent to participate in employee programs (work behavior).

\section{Cultural Values}

In cross-cultural research there are substantial variances acknowledged in cultural characteristics between eastern and western cultures (Hofstede, 1980, 1994; Lok \& Crawford, 2004). Yang, Wang and Drewry (2009) defined culture "as a collectively shared social knowledge and understanding that is reflected in values, beliefs and habitual social norms within a group of individuals" (p.325). According to Hofstede, Neuijen, Ohayv and Sanders (1990), "culture is that complex whole which includes knowledge, beliefs, art, morals, law, customs and any other capabilities and habits acquired by man as a member of society" (Taylor, 1924, p.1; p.311). Hofstede (2001) further defined culture as the "collective programming of the mind that distinguishes the members of one group or category of people from another" (p.9).

Research on culture cannot be completed in a vacuum, and thus cultural research encompasses ethnicity and minority. "Ethnicity is based on a social construction of an origin as a basis for community or collectivity: 'this origin, mythical or 'real', can be historically, territorially, culturally, or physiognomically based" (Anthias \& Davis, 1992: 423). According to Kamenou (2010), ethnicity comprises a number of factors, "including cultural and racial characteristics and it is viewed here as broader than 'race' encompassing cultural diversity, values and traditions" (p.1999). Ragins (1997) posited that sociologists define minority in terms of power relations among groups (ac Blalock, 1967; van Amersfoot, 1978). Furthermore, "Baker (1983) suggested that race and ethnic relations are viewed and analyzed in terms of intergroup power relations" (ac Ragins, 1997, p.486), with power being defined in terms of group 
interactions and/or influential resources. Lok and Crawford (2004) suggested that national cultures affect employee behavior; moreover, employees' expectations and perceptions differ with regard to national cultures.

According to Gerhart and Fang (2005), "Dowling, Welch, and Schuler (1999) note that, '[b]ecause international business involves the interaction and movement of people across national boundaries, an appreciation of cultural differences and when these differences are important is essential" (p.12; p.973). Blake-Beard, Murrell, and Thomas (2007) suggested it is critical for organizations to understand interactions across different racial groups due to the changing nature of organizations and the composition of the people within them. Gerhart and Fang (2005) believed that culturally insensitive attitudes and behaviors are inappropriate and occur due to ignorance or misguided beliefs, and in some cases, can cause business failure. According to Caproni (2005), "Organizations must grapple with how to engage this diverse population in a common enterprise among disparate groups who "do not share a common history or culture" (p. 269).

Due to the globalization of business and the implications globalization brings to management and organizations, Gerhart and Fang (2005) believed that organizations should increase attention to country differences. "Firms must address how to support and enable relationships among people who come from diverse cultures, backgrounds, and perspectives" (Blake-Beard, Murrell, \& Thomas, 2007, p.224). According to Murrell and James (2001), the changing composition of the workforce means that individuals will experience more cross-race (and cross-cultural) interactions within organizations. Kelly (2008) posited this is a positive change, as through assembling and sustaining a culturally-competent workforce, an organization may gain a competitive advantage over its rivals (Sparrow, Schuler, \& Jackson, 1994; Tayeb, 1995; Wright, McMahan, \& McWilliams, 1994). Values, attitudes and beliefs are fashioned by 
one's national cultures, and as such, most individuals bring their personal values, attitudes and beliefs to the workplace, which has an effect on the existing organizational culture and more than likely, will influence how organizations are managed (Lok \& Crawford, 2003).

Gerhart and Fang (2005) posited "national culture almost seems at times to be deterministic of management practice" (p.973). In fact, researchers have established individual behavior is influenced by national culture, and as such, there are substantial differences between eastern and western cultures, thus in turn, significant dissimilarities in management in these culturally specific organizations (Hofstede, 1980; Trompenaars \& Hampden-Turner, 1998). McGuire, O'Donnel, Garavan, Saha, and Murphy (2002) suggested influences such as religion, ethnicity, education, and geography contribute to the classification of a definite culture. Hofstede et al. (1990) stated, "values are acquired in early youth", and as culture is namely the values of a collectivity, "the degree that values of individuals and of collectivities (culture) differ across nations" influence how people respond, including management practices (p. 312).

Morden (1995) suggested in order for organizations to be prosperous in international locations, the organization must understand and appreciate the cultural milieu in which they will be operating. Newman and Nollen (1996) found offices employing management styles fitting the cultural contexts were more financially productive than those offices whose management practices did not fit with the cultural background. National culture differences can be precarious and as such, insensitivity and inattentiveness to national culture differences can and does result in business failure (Gerhart and Fang, 2005). According to Gerhard and Fang (2005), "leading texts on international management warn that national culture differences matter a great deal and a manager ignores them at his or her own peril" (p.973). An organization may comprise numerous culturally diverse departments which may consist of culturally-rich groups. Gerhart and Fang (2005) posited "a misfit between national culture and management practice will reduce 
effectiveness" (p.974). Barney (1999) suggested an organization with a unique culture, not indicative or relative to the country or industry can be risky, but it can also be advantageous. Hofstede (1983) stated the importance of 'nationality' on a culture's spirit such that "national and regional differences are felt by the people to be a reality and therefore they are a reality" (Kelly, 2008, p.75). These 'realities' affect performance and attitudes. According to Kelly (2008), "Nations impart a symbolic value of belonging and common identity to their respective citizens. These differences are seen as reality by the citizens, and therefore, Hofstede surmises, are reality" (p.14). Gerhart and Fang (2005) concurred and suggested differences in culture due to country or region are greater than organizational culture differences.

"Between-country differences in culture are large relative to within-country differences and national differences in culture (and in HR practices) are large relative to differences due to other factors such as occupation or organization differences" (Gerhart \& Fang, 2005, p.974). In fact, according to Smith, Bond, and Kagitcjbas (2006), even a communal nationality does not create cultural homogeneity as factors, including ethnicity, religion, class, and education can be the foundation for numerous subcultures within a single national context. Adler (2002) explained, "diversity exists both within and among cultures; however, within a single culture certain behaviors are favored and others repressed; moreover, a cultural orientation describes the attitudes of most people most of the time, never all of the people all of the time" (p.18-19).

According to Hofstede, Neuijen, Ohayv and Sanders (1990), the four-dimensional model of national culture differences certainly does not epitomize the definitive truth about culture, but it has so far served as a useful framework for guiding research design in the field of national cultures. Hofstede's key postulation is that the values of individuals within a nation [region] are (significantly) more akin than the values of individuals from different nations [regions]. 
Yang, Wang and Drewry (2009) suggested using Hofstede's framework of culture as "Hofstede's dimensions are one of the widely accepted and well-studied sets of cultural dimensions" (p.325). Geert Hofstede's 1980 book, Culture's Consequences, utilized international data on IBM employees to cultivate four national culture dimensions. Hofstede and McCrae (2004) summarized Hofstede's multinational research:

"Hofstede's study of national culture differences used a database collected by a multinational corporation (IBM) in its subsidiaries in 71 countries, containing the scores on a series of employee attitude surveys held between 1967 and 1973, a total of around 117,000 questionnaires. These surveys had explicitly tried to tap the employees' basic values along with their situational attitudes. IBM in those days was a tightly structured organization with a unified set of products and product-related jobs and a strong corporate culture, meaning that samples of employees from one subsidiary to another could be strictly matched to be similar in all respects except nationality" (p.61).

Hofstede's (1980) quantification of culture proposed and measured four dimensions of national culture: Individualism (IDV), Power Distance (PDI), Uncertainty Avoidance (UAI), and Masculinity (MAS). Hofstede and Bond (1988) added an "additional dimension independent of the original four, "Confucian dynamism" opposing a long-term to a short-term orientation in life and work" (Hofstede et.al, 1990, p. 288); hereby referred to as Long Term Orientation (LTO). Using Hofstede's dimension, "Hofstede demonstrated that cultural variations between nations do exist and that they have the real potential to affect differences in HRM practices and perceptions" (Kelly, 2008, p.12). Hofstede posited that nationality is a formative factor in determining perceptions. 


\section{Power Distance}

According to Hofstede and McCrae (2004), "power distance is the extent to which the less powerful members of organizations and institutions (such as the family) accept and expect that power is distributed unequally" (p.62). Emery and Oertel (2006) posited power distance specifies how different societies have established, addressed and endorsed basic human inequalities in social status and esteem, prosperity, and sources of power. Power distance embodies inequality and advocates that "all societies are unequal, but some are more unequal than others" (Hofstede \& McCrae, 2004, p.62).

Kelly (2008) described power distance as a scale to which members of a society accept an unequal distribution of power between other members and organizations. Hofstede (1994) characterized small power distance countries by a "limited dependence of subordinates on bosses and a preference for consultation, that is, interdependence between boss and subordinate" (p. 27). In large power distance countries, due to the emotional distance between subordinate and supervisor, the subordinate is less likely to request advice from their supervisor directly. According to Clutterbuck (2007), "High power distance cultures may tend to favor the sponsorship model, but this may clash with the cultural expectations of multinationals dominated by low power distance countries, such as Northern Europe" (p.646).

Lok and Crawford (2003) suggested it is widely recognized that Chinese firms feature high power distance values and possess a bureaucratic culture where there is minimal delegation and empowerment; moreover, leadership in Asian firms is predisposed based on authority and seniority. Chen and Francesco (2000) suggested due to the cultural nomenclature in China, organizational commitment is highly associated with loyalty to the organizational leaders. In as much, Lok and Crawford (2003) proposed that Asian organizations could augment employees' 
commitment and generate greater job satisfaction utilizing a patriarchal and paternal mentoring approach.

\section{Uncertainty Avoidance}

According to Hofstede and McCrae (2004), uncertainty avoidance deals with a "society's tolerance for ambiguity" as uncertainty avoidance designates how culture influences members to feel either uncomfortable or comfortable in unstructured situations, which are defined as "novel, unknown, surprising, and different than usual" (p.62). Hofstede and McCrae (2004) continued and suggested uncertainty-avoiding cultures "try to minimize the possibility of such situations by strict laws and rules, by safety and security measures, and, on the philosophical and religious

level, by a belief in the absolute Truth: 'The can only be one Truth and we have it.'"(p.62). Kelly (2008) utilized the Hofstede (2001) definition to describe uncertainty avoidance as a measure of how members of a culture feel vulnerable or anxious either in or by the indication of unfamiliar situations or uncertain outcomes. Claes and Ruiz-Quintanilla (1998) also used Hofstede (1991) to define uncertainty avoidance as "the extent to which the members of a culture feel threatened by uncertain or unknown situations" (p.113; p.363). Emery and Oertel (2006) posited that the uncertainty avoidance element represents the degree to which a society is disinclined to accept and handle uncertainty.

Hofstede (1994) asserted this aspect of culture relates to an individuals' acceptance of anxiety, their need for security, their reliance on authority, and the dependence and solicitation of evidence. In 2001, Hofstede furthered his claims that individuals in uncertainty-avoiding countries are emotional, are motivated by inner nervous energy, and have a plethora of rules and laws; whereas individuals in uncertainty-accepting cultures are more tolerant of differing viewpoints and opinions of others, tend to have fewer rules, and as philosophical vacillators, 
uncertainty-accepting individuals, are less inclined to express emotions. According to Claes and Ruiz-Quintanilla (1998), "Workplace related characteristics for weak uncertainty avoidance include tolerance of deviant and innovative ideas and behavior and motivation by achievement and esteem or belongingness; [moreover], strong uncertainty avoidance has to do at the workplace with suppression of deviant ideas and behavior, resistance to innovation, and motivation by security, esteem, and belongingness" (p.363).

\section{Individualism/Collectivism}

Hofstede and McCrae (2004) described individualism and collectivism in terms of group integration of individuals. For instance, in individualistic societies, the connection between individuals are loose in such a manner that individuals are "expected to look after himself or herself and his or her immediate family; [whereas], in collectivistic societies, people are integrated from birth onward into strong, cohesive in-groups, often extended families (with uncles, aunts, and grandparents), protecting them in exchange for unquestioning loyalty" (Hofstede, 1994, p.51; p.63). Kelly (2008) concurred with Hofstede’s 2001 definition and suggested "individualism, and its corresponding opposite collectivism, measures the strength of ties between a society's members and their fellow members" (p.12). Emery and Oertel (2006) elucidated the individualism facet reflects the way society encourages individual decisionmaking and behaviors. "According to Schwartz (1990), collectivist societies are communal societies characterized by diffuse and mutual obligations and expectations based on ascribed statuses" (e.g., Triandis, 1995; Oyserman, Coon \& Kemmelmeier, 2002, p.5).

Hofstede (1980) believed that "because in-groups can include family, clan, ethnic, religious, or other groups, Hui (1988) and Triandis (1995), among others, have proposed collectivism is a diverse construct, joining together culturally disparate foci on different kinds 
and levels of referent groups. In this way, collectivism may refer to a broader range of values, attitudes, and behaviors than individualism" (Oyserman, Coon \& Kemmelmeier, 2002, p.5). Oyserman, Coon and Kemmelmeier (2002) utilized Hofstede's 1980 definition of individualism and suggested individualistic cultures emphasize personal sovereignty and self-fulfillment, and base their identity on personal accomplishments.

Drost, Frayne, Lowe and Geringer (2002) suggested organizations resemble a family structure with mutual obligations of protection in exchange for loyalty. According to Claes and Ruiz-Quintanilla (1998), "Individualism has to do with personal time, freedom, and challenge, [and as such], in the workplace it is characterized by the employees being rather independent from the organization and taking care of themselves"(p.362). Oyserman, Coon and Kemmelmeier (2002) suggested that individualism culture promotes a decontextualized reasoning style. Drost, Frayne, Lowe and Geringer (2002) posited that collectivist cultures focus on group member skills, such that, collectivist individuals perceive training as an organizational benefit which therefore, reinforces the employee's organizational dependence. Furthermore, Claes and Ruiz-Quintanilla (1998) stated that collectivism societies, in regards to training and the use of skills, create a working environment where in-group relationships and dealings prevail over task. Emery and Oertel (2006) suggested that "employees with a lower individualism Index (IDV) have lower career aspirations and tend to have a high emotional dependence and a high moral involvement in the company. Employees with higher individualism scores have a higher career orientation and egoistic working style which often tend to reject follower ideas and initiatives" (p.4-5). 


\section{Masculinity/Femininity}

Hofstede and McCrae (2004) described masculinity versus its opposite, femininity as the "distribution of emotional roles between the sexes", such that "the assertive pole has been called 'masculine' and the modest, caring pole "feminine" (p.63). Hofstede (1980) theorized "masculinity pertains to societies in which social gender roles are clearly distinct, [and] femininity pertains to societies in which social gender roles overlap" (p. 82). Kelly (2008) posited that "masculinity, or femininity, refers to how well defined gender roles are within a culture as well as the level of objectivity used to measure outcomes or performance" (p.13).

Emery and Oertel (2006) delineated the masculinity dimension within a culture was dictated by the importance of "traditional male values, such as assertiveness, performance, ambition, achievement, and materialism, to a society. The opposite end of this continuum has been labeled femininity" (p.6). Kelly (2008) differentiated between the two cultures such that "a more masculine culture would be characterized as one in which men and women have clearly separate jobs, duties, and emotions; [whereas], in a feminine culture, these roles and emotions would be blurred or shared between men and women" (Hofstede, 2001; p.13).

According to Claes and Ruiz-Quintanilla (1998), "masculinity has to do with earnings, recognition, and opportunity for advancement to higher level jobs, challenging work, competition among colleagues, and performance," (p.363) and so, it is assumed that career planning is fundamental to masculine national cultures. In contrast, Claes and Ruiz-Quintanilla (1998) suggested that "femininity has to do with a good working relationship with direct superiors, work with people who cooperate well with one another, living area, employment security, and the quality of working life," (p.363) and so, it is assumed that relationships are significant to feminine national cultures. Whereas masculinity inhibits preemptive career 
behaviors, femininity facilitates career development behaviors such as building relationships and networking activities.

\section{Long-Term/Short-Term Orientation}

Added in the 1980s, the Long-Term Orientation (also referred to as the Confucian Dynamism dimension) dimension was added in order to study long-term versus short-term orientation. This dimension was based on a questionnaire designed by Chinese scholars utilized in a study among students in 23 countries around the world (Hofstede \& Bond, 1988). Hofstede and McCrae (2004) stated that "values associated with long-term orientation are thrift and perseverance; values associated with short-term orientation are respect for tradition, fulfilling social obligations, and protecting one's face" (p.63). As of 2004, this dimension has only been applied based upon the Hofstede and Bond 1988 study.

\section{Country-Level Comparisons}

Chao (2007) simply defined culture - "as implicit and explicit values and understandings shared by a group of people" (p.188). Hofstede (1991) defined national culture as the communal indoctrination that differentiates the members of one nation from another. "Hofstede's operating definition is "the collective programming of the mind that distinguishes one group or category of people from another". This stresses that culture is (a) a collective, not individual, attribute; (b) not directly visible but manifested in behaviors; and (c) common to some but not all people" (Hofstede \& McCrae, 2004, p.58)

Chao and Moon (2005) suggested that aside from general conceptualizations of culture tied to nationality or ethnicity, other cultures may be identified from demographic, geographic, and associative features. Chao (2007) stated associative features of cultural identities designate 
that which an individual deems important, such as an individual's professional identity. Hofstede (1991) believed national culture-level values can affect personal values, attitudes and behaviors. According to Claes and Ruiz-Quintanilla (1998), "in addition to politics and socioeconomics, national culture will influence individuals' careers at a macrosocial level” (p.357).

According to Emery and Oertel (2006), one must understand how an employee processes his or her environment to make choices, and as such, a “person's perception is thought to be a major contributor to one's motivation and an excellent predictor of behavior" (Henle, 2005; p.3). Emery and Oertel (2006) suggested that "the perception construct is based on an individual's attitude, personality, values, beliefs and norms" (Allport, 1955; Freud, 1963; p.3).

Per se, understanding of one's cultural-based perception might be a strong predictor of motivation and behavior. According to Hofstede and McCrae (2004), "the study of personality and culture is no longer a matter of documenting how culture shapes personality; instead, it asks how personality traits and culture interact to shape the behavior of individuals and social groups" (McCrae, 2000; p.57). When we compare individuals, we study personality; when we compare societies, we study culture. "Hofstede does not claim that trait levels are completely determined by cultural influences, and McCrae does not suppose that cultural values are merely a reflection of personality" (p.69-70). In the international data, according to Sadler and Hofstede (1972), "there is considerable difference from country to country and from job to job and preferences and perceptions may be influenced by: (1) the cultural environment in which an employee is brought up, depending on his country, social class and, maybe, sex" (p.56).

According to Clutterbuck (2007), "very little comparative study has been carried out regarding cultural differences (Barham \& Conway, 1998, is one exception), but the principle issues reported by multinational companies in our program design activities seem to be captured in the theories of Hofstede (1991) and Trompenaars (1993) concerning the primary components 
of cultural difference" (p.646). Sadler and Hofstede (1972) suggested that "the greatest differentials are between preferences in the countries with the strongest differences in cultural backgrounds, like U.K., Japan, and Brazil” (p.60).

Wever (1995) suggested that there are many national and organizational, cultural and structural variables at play. According to Oyserman, Cook and Kemmelmeier (2002), countrylevel comparisons necessitate vast resources of groups, thus enabling the researcher to sample a sufficient number of distinct groups for quantitative analyses; moreover, for generalizability, the resources also allow for these groups to be representative of the society overall. In 1980, Hofstede's conducted his groundbreaking survey of IBM employees in 39 nations, from which, he generated country-level indicators, conceptualizing four cultural constructs as a function of workplace values. According to Oyserman, Cook and Kemmelmeier (2002), "Hofstede (1980) reviewed possible antecedents and implications of these job-relevant values for societies, [and his] model was important because it organized cultural differences into overarching patterns" (p.3). Hofstede $(1980,1994)$ and numerous other researchers consider his constructs and their associated findings still relevant today.

\section{Cultural Values [Diversity] and Mentoring}

To date, according to McKeen and Bujaki (2007), "relatively little is known about race and mentoring (Blake-Beard, Murrell, \& Thomas, Ch. 9, this volume; Kalbfleisch \& Davies, 1991), and less is known about the interaction of gender and race in mentoring relationships" (p.203). Blake-Beard, Murrell, and Thomas (2007) posited that race is a socially embedded phenomenon that touches all aspects of our lives, and as such provides a valuable lens with which to examine the mentoring literature (Alderfer \& Thomas, 1988). Wever (1995) suggested 
that with the realities of international competition, organizations not incorporating a multinational strategy will struggle.

Ragins (1997) believed "a link between diversity and mentorship can also help expand and define the newly emerging field of mentorship in organizations" (p.483). Multinational companies introducing mentoring programs around the world have attempted a variety of strategies. Noe, Greenberger, and Wang suggested that "most of the research on diversified mentoring relationships has considered diversity from the perspective of age, gender, race, and nationality" (p.147). According to Ragins (1997), "diversified mentoring relationships are comprised of mentors and protégés who differ in group membership associated with power differences in organizations (e.g. race, ethnicity, gender, class, disability, sexual orientation)" (Ragins, 1995; p.482).

"Research on cross-race relationships reveals distinct and important differences in mentoring processes and outcomes (Atkinson, Neville, \& Casas, 1991; Burke, McKeen, \& Mckenna, 1993; Thomas, 1990, 1993)" (p.482). Little is known about the types of benefits and barriers that mentors and protégés experience in mentoring relationships that are cross-cultural.

Ragins (1997) stated "it is important to consider the degree of diversity within mentoring relationships" (p.490). Within mentoring research, Blake-Beard, Murrell, and Thomas (2007) suggested, "it is inappropriate to assume that the experiences of one group adequately and accurately capture the experiences of other groups" (p.235). According to McKeen and Bujaki (2007), "Wilson and Elman (1990) noted that the increasing diversity of the workforce adds another dimension to the mentor-protégé matching problem: difficulties inherent in the establishment of relationships between people who do not closely identify with one another" (p.202). According to McKimm, Jollie and Hatter (1999, 2003, 2007) suggested organizations should prepare for "cultural mismatch; race mismatch" (p.13). 
Ibarra (1995) suggested that social networks are impacted by race, which in turn, impacts the outcomes of mentoring relationships. "A key challenge for future research on mentoring is to move beyond the faulty assumption that the experience of race within organizations does not shape, alter, and drive the mentoring relationship" (Blake-Beard, Murrell, \& Thomas, 2007, p.235). Scholars advance that a challenging area ripe for research concerns the fundamental issue of how we define mentoring within the context of race. "Dickens and Dickens (1982) argued that people of color (specifically African Americans) are often not able to reap the benefits of mentoring relationships because the issue of race and the dynamics of race relationships act as critical obstacles" (Blake-Beard, Murrell, \& Thomas, 2007, p.231).

The changing organizational environment, with regard to race and culture dynamics, create an absence in the mentoring literature, as most highly regarded models, theories, and empirical studies either exclude race as a factor or include samples that lack diversity such that race is relegated to 'unexplained variance' (Blake-Beard, Murrell, \& Thomas, 2007). Lankau and Scandura (2007) suggested that "protégés in diversified relationships (e.g. cross-gender and cross-race) do not experience the same levels of vocational and psychosocial support for protégés in homogeneous relationships report" (p.112).

Moreover, according to Ehrich, Hansford and Tennent (2001), "several studies within the business database reported that misunderstandings or incompatibility associated with gender or race hampered the success of the mentoring relationship" (p.10). One of the studies that investigated the influence of nationality on mentoring found that differences in nationality of the mentor and protégé can have an influence on the amount of mentoring the protégé receives, namely, they found that it would be less likely for the protégé to receive mentoring if their nationalities were dissimilar to the mentors (Feldman, Folks, \& Turnley, 1999). According to Parise and Forret (2008), "there is a better chance to form a relationship when personalities mesh 
(Kram \& Hall, 1996) and when two people see their own similarities and reasons why they may get along; a poor match [mismatch] can cause the mentor to resent his or her involvement (Kram \& Hall, 1996) and ultimately provide fewer mentoring functions for the protégé (Allen, Eby, \& Lentz, 2006)" (p.228)

According to Turner (1982), "culture can be a source of social identity and a catalyst for two people to initiate a relationship" (Chao, 2007, p.188). Egan and Song (2008) posited that "the success of the mentor-protégé relationship is dependent on early interpersonal connections (perhaps influenced by perceived similarity; Allen et al., 2004; Lee, Dougherty, \& Turban, 2002)" (p.353). Ozkalp, Kirel, Sungur, and Ozdemir (2008) found that a major obstacle in mentor and protégé interaction was not having the same cultural background with their counterpart. If individuals come from different backgrounds, cultures and even, generations, they may not understand or empathize with each other. Ensher and Murphy (1997) suggested similarity is an important consideration in dyad pairing. Thus, the degree of similarity between the mentor and the protégé, either actual or perceived, could affect the quality of the mentoring relationship (Turban \& Jones, 1988; Ensher \& Murphy, 1997, p.463).

Mentoring becomes even more complicated when the mentoring programs match people across cultures, especially if they are living in different countries. According to Matuszek, Self and Schraeder (2008):

"An additional issue relates to the psychological nature of the mentoring relationship. Individuals process and interpret information in different ways, based on our personalities, our life experiences, national culture, and educational level. Often, in the traditional organizational environment, the mentor and protégé share, by virtue of being in the same location, certain similarities that are the result of absorbing local values and norms, even if the mentor and 
protégé come from very different backgrounds. When the mentor and protégé are thousands of miles apart, even though they may work for the same corporation, there is no monolithic culture. Rather, there is a series of subcultures. Thus, if these barriers of differing values and norms hamper effective feedback, the likelihood of confusion or misinterpretation of information is increased" (p.19).

According to Lankau and Scandura (2007), "diversified mentoring relationships may result in specific learning outcomes, such as knowledge about minority group members that dispel stereotypes; increased understanding about the unique career needs of minorities and barriers to advancement; and the development of empathy and communication to bridge cultural, ethnic, and gender gaps" (p.117). Though it is intuitive to think that in multinational corporations having a mentor who is of the host-country nationalilty may help the protégé (i.e. expatriate) who is of different a nationality understand the culture better, Feldman et al (1999) showed through their study that it may be more beneficial if the mentor and the protégé are of the same nationality, because sharing the same culture background facilitates effective communication.

\section{Mentoring}

According to Betts and Pepe (2006), "Mentoring is a sustained relationship between a younger, less experienced individual (protégé) and an older, more experienced individual (mentor) dedicated to achieving long term success and fulfillment" (Burke, 1984; Kram, 1985; p.105). Hagenow and McCrea (1994) focused on the time-honored concept of mentoring and suggested that "mentoring is first and foremost a teacher/student relationship" (p.42, a.c. Armstrong, Allinson, \& Hayes, 2002, p.1112). "Mentoring is one of the oldest forms of human 
development" (Shea, 1994, p.13). Gibbons (2000) defined mentoring as "a protected relationship in which learning and experimentation can occur, potential skills can be developed, and in which results can be measured in terms of competence gained rather than curricular territory covered" (p.18). Godshalk and Sosik (2007) suggested that "mentoring involves a long-term role model relationship that is professionally and personally development oriented" (p.155); moreover, “traditional definitions of mentoring assume mentor and protégé exist within the same organization" (p.160). "Traditionally, mentoring has been defined as an intense interpersonal exchange between a senior experienced colleague (mentor) and a less experienced junior colleague (protégé) in which the mentor provides support, direction, and feedback regarding career plans and personal development" (Russell \& Adams, 1997, p.12)

Megginson and Clutterbuck, (1995) defined mentoring "as help by one person to another in making significant transitions in knowledge, work or thinking" (p.3). Mullen (1994) described mentoring as a reciprocal exchange of information in which both the mentor and the protégé solicit knowledge from one another. According to Ozkalp, Kirel, Sungur, and Ozdemir (2008), "Mentoring is therefore crucial for both individual and organizational development."(p.67). According to Eby, Allen, Evans, Ng, and DuBois (2007), "when a more experienced or senior individual (the mentor) takes an interest in and encourages a less experienced or disadvantaged individual (the protégé), the protégé will benefit" (Jacobi, 1991; Kram, 1985; Rhodes, 2005; p. 255).

Baugh and Fagenson-Eland (2007) posited that "given all of the potential benefits of mentoring, it is not surprising that organizations have begun to develop mentoring programs in order to ensure that such advantageous relationships are developed and maintained, rather than relying on happenstance" (Barbian, 2002a; p.249). In fact, according to Rosser (2004), more than one third of large companies in the United States use mentoring to develop their employees, 
as organizations realize that mentoring advances the development of social capital (Lankau \& Scandura, 2007). According to Bozionelos (2002), "mentoring has been established as a human resource practice and as an individual strategy for career success" (p.25)

According to Gentry, Weber and Sadri (2008), "Mentoring is a relationship that focuses on collaboration, development, and feedback" (p.249). Mentoring is a substantial practice that creates a relationship between mentors and mentees that fosters learning and growth. Wanberg et al. (2003) posited that "mentoring is the most intense and powerful one-on-one developmental relationship, entailing the most influence, identification, and emotional involvement" (p.5). Clutterbuck and Megginson (1999) concurred and stated that mentoring "can evoke strong feelings both for the people involved and those looking on from the outside" (p.162). According to Gentry, Weber and Sadri (2008), "Mentoring is not an 'all or nothing' experience and frequently has a select number of career-related functions at a given time (Bozionelos, 2004; Kram, 1985; Ragins, Cotton \& Miller, 2000)" (p. 246). "Mentoring is a powerful intervention, but it is not magic (Clutterbuck \& Megginson, 1999, p.160). In order for mentoring to work, the parties involved need to spend abundant time in developing a relationship, establishing goals and discussing issues to be explored and topics to be learned.

According to Eby, Allen, Evans, Ng, and DuBois (2007), "workplace mentoring occurs in an organizational setting and the purpose is the personal and professional growth of the protégé" (Kram, 1985, p.255). Eby (1997) suggested that a mentor may be a supervisor, an individual within the organization but outside the protégés work hierarchy, or an individual in another organization. Ozkalp, Kirel, Sungur, and Ozdemir (2008) posited that "mentoring is a learning centered process for both the mentees and the mentor and has been defined as a one to one, non-judgmental relationship in which an individual voluntarily and professionally gives time to support and encourage another" (p.75). According to Eby, Allen, Evans, and DuBois 
(2007), "workplace mentoring aims to enhance employees' personal and career development (Kram, 1985; p.257)."

Kram (1985) suggested that "mentoring can significantly benefit both individuals and organizations. For individuals in early career, mentoring can reduce the shock of organizational entry and help prepare for advancement (Levinson, et al., 1978; Dalton, et al., 1977). For individuals at midcareer and beyond, mentoring can help them meet generative needs, stay in touch with their youthful sides, stay abreast of technological advances, and attain confirmation by passing on wisdom and experience" (Levinson, et al., 1978; Hall \& Kram, 1981; Dalton et al., 1977; p.159). Scandura and Siegel (1995) found mentoring could improve a new employees' adjustment to organizational change by aiding employees in acquiring skills associated with adaptability. Moreover, mentoring relationships provide both mentors and protégés with an opportunity to gain a new understanding of their identities, values, and self-worth (Kram, 1985).

\section{Definition of Mentor}

McKimm, Jolie and Hatter $(1999,2003,2007)$ affirmed mentoring is a very old concept in a new guise. According to Ensher and Murphy (1997), "Mentoring is an age-old developmental tool" (p.460). Mentoring can be traced back to Greek mythology. The origin of the word 'mentor' can be traced back to the Greek meaning 'enduring' and was first used in Homer's epic, The Odyssey, written around 700 BC. In Homer's story, Odysseus, the King of Ithaca entrusted his son Telemachus to the Goddess Athena, who disguised herself in human form as Mentor. Mentor was the friend and servant of Odysseus, entrusted with the education of his son Telemachus during the Trojan War (Hamilton, 1942). Mentor's function - according to Homer - was to act as a wise counselor and helper to Telemachus. According to Ehrich, Hansford, and Tennent (2001), since 700 BC, and particularly during the last thirty years or so of 
the last century, much has been written about mentoring and its potential for enhancing workplace learning and developing an organization's human resources" (p.3).

Recounted by Ehrich, Hansford, and Tennent (2001), "The generic meaning of a mentor is a 'father' figure who guides and instructs a younger person" (p.3). In ancient Greece, it was a common practice for wise men to counsel younger men, and Mentor became the 'counselor, guide, tutor, coach, sponsor, and mentor for his protégé' (Hunt \& Michael, 1983).

Ragins (1997) articulated that "traditionally mentors are defined as individuals with advanced experience and knowledge who are committed to providing upward mobility and support to their protégés' careers" (Hunt \& Michael, 1983; Kram, 1985; p.484). Allen, Poteet, Russell and Dobbins (1997), posited that "mentors are persons usually considered as more experienced, who support, train, 'teach the ropes to' or sponsor others as they pursue their career goals" (p.9). Rosser (2004) utilized Merriam-Webster's Collegiate Dictionary (1993) and defined a mentor as "a trusted counselor or guide" (p.726; p.3). Definitions of the term 'mentor' typically contain phrases like: "a seasoned executive" (Olian, Carroll, Giannantonio, \& Feren, 1988); "a trusted and experienced guide" (Johnson, 1980); "more powerful executive" (Bowen, 1982); "a person committed to providing upward career mobility" (Dougherty \& Dreher, 2007); 'A mentor is 'a trusted counselor or guide' (Locurcio \& Mitvalsky, 2002). Fagenson (1989) described a mentor as "someone in a position of power who looks out for [the protégé], or gives [the protégé] advice, or brings [the protégé's] accomplishments to the attention of other people who have power in the company" (p.312).

Donaldson, Ensher, and Grant-Vallone (2000), defined a mentor as “a more experienced employee who advises, counsels, or otherwise enhances the personal development of a less experienced employee” (p.238). Ragins, Cotton and Miller (2000) advocate Kram's 1985 definition in that "mentors are generally defined as individuals with advanced experience and 
knowledge who are committed to providing upward mobility and career support to their protégés" (p.1178). Moreover, Ragins, Cotton and Miller (2000) used an established definition of mentor (Ragins, 1989): 'A mentor is generally defined as a higher-ranking, influential individual in your work environment who has advanced experience and knowledge and is committed to providing upward mobility and support to your career" (p.1182).

Perhaps a more appropriate definition of a mentor is: anyone who provides guidance, support, knowledge, and opportunities for an individual who possesses less knowledge and needs direction in order to reach their goals. Burlew (1991) suggested "a mentoring relationship exists because one person is helping another person progress through life" (p.214). As set forth by McKimm, Jolie and Hatter (1999, 2003, 2007), mentors bring experience, knowledge and objectivity into the mentoring relationship as they offer a wider perspective of the organization for the individual. According to Donaldson, Ensher, and Grant-Vallone (2000), "mentors often offer good relationships as protégés' colleagues or supervisors, provide feedback, and help socialize protégés to an organization's norms” (p.235). McKimm, Jolie and Hatter (1999, 2003, 2007), suggested mentors should help the protégé reach their goals and mentors "should also be willing to share their expertise, should not feel threatened by the mentee's potential for equaling or surpassing them nor by the protégé detecting their weaknesses and shortcomings" (p.4).

\section{Definition of a Mentoring Program}

Researchers throughout mentoring literature employ many definitions of mentoring. According to Ensher and Murphy (1997), “descriptions range from somewhat simple dyadic relationship in which an older individual coaches, guides, and helps a protégé (Hunt \& Michael, 1983 ) to a relationship between two individuals whose nature changes over time (Kram, 1985)" (p.460-461). The Mentoring Program Handbook (2003) suggested that "the success of the 
mentoring relationship depends greatly on how well the mentoring relationship is defined." Ragins and Kram (2007) posited that "although the definition of mentoring has been refined over the years, a core feature that defines mentoring relationships and distinguishes [it] from other types of personal relationships is that mentoring is a developmental relationship that is embedded within the career context" (p.5). Thomas (1990) suggested the term "development relationship is one that provides needed support for the enhancement of an individual's career development and organizational experience" (p.480). Scandura and Williams (2001) submit "a mentor can generally be defined as an influential individual in your work environment who has advanced work experience and knowledge and who is committed to providing upward mobility and support to your career" (p. 349).

Noe, Greenberger and Wang (2002) advocate "mentoring has been defined as an intense personal exchange between a more senior, experienced, and knowledgeable employee (i.e. the mentor) who provides advice, counsel, feedback, and support related to career and personal development and less experienced employees" (i.e. the protégés) (Hunt \& Michael, 1983; Kram, 1985; Noe, 1998a; Russell \& Adams, 1997; p.130). Furthermore, Locurcio and Mitvalsky (2002), defined mentoring as "a relationship whereby a person with greater experience and wisdom guides another person to develop both personally and professionally" (p.32). According to Mattei (2001), "Mentoring in an organization is a private relationship between two individuals based on a mutual desire for development toward an organizational objective" (p. 44).

According to Ragins and Cotton (1999), formal and informal mentoring relationships differ with regard to the structure of the relationship. Rosser (2004) described informal mentoring as a "spontaneous and voluntary" relationship that is "loosely structured" (p.31). A formal mentoring partnership contains a comprehensive strategy which includes a facilitated matching process, formal training and clear goals for measuring success. Rosser (2004) 
described that formal mentoring relationships form through the organization, are "developed through a third party", and are designed with "identifiable and specific outcomes"; moreover, the relationship has a "set time frame and often comes with extrinsic rewards" (p.31-32). This study exclusively examined mentoring that occurs within the context of a formal program and, therefore, the following definition by Bowen (1985) is used: Mentoring occurs when a senior person (the mentor) in terms of age and experience undertakes to provide information, advice, and emotional support to a junior person (the protégé) in a relationship which is set formally by the constraints of the program and lasts for a limited period of time" (p.31).

\section{Formal Mentoring}

According to the Mentoring Program Handbook (2003), formal mentoring is appealing to most organizations because it involves employees, not only in their own growth and development, but also in the growth and development of the organization. Tyler (1998) identified formal mentoring as an evolving movement in HRD programs. As such, Bragg (1989) posited a third of the nation's major companies utilize formal mentoring programs and Noe, Greenberger and Wang (2002) concured, stating "Douglas and McCauley (1999) "found that $20 \%$ of organizations had a least one formal program in place" (p.153). According to Ragins, Cotton and Miller (2000), "formal mentoring relationships develop with organizational assistance or intervention, which is usually in the form of matching mentors and protégés" (p.1177).

Formalized mentoring programs are a focused initiatives utilized by organizations to aid employees with personal job endeavors, yet are flexible enough to be tailored to each individuals own developmental needs. "The formality of the plan will depend on the work atmosphere, but it should be divided into four parts: the business environment, technical responsibilities, business 
practices, and training requirements. It is important that this plan be developed jointly by the mentor and the person being mentored and include discussions with or approval by the employee's supervisor" (Locurcio \& Mitvalsky, 2002, p.31-32).

According to the Mentoring Program Handbook (2003), "A formal mentoring program is not a one-shot training event." In fact, throughout the literature, a formal mentoring program is referred to as a "facilitated process", created by organizations to occasionally orient new employees (Murray, 1991), but formal mentoring mainly seeks to utilize the intellectual capital within the organization, in addition to encouraging professional development amongst employees that coincides with the strategic goals of the organization. Scandura and Schriesheim (1994) conceptualized supervisory mentoring "as a transformational activity involving a mutual commitment by mentor and protégé to the latter's long-term development, as a personal, extraorganizational investment in the protégé by the mentor, and as the changing of the protégé by the mentor, accomplished by the sharing of values, knowledge, experience, and so forth (Hunt \& Michael, 1983; Kram, 1985)”(p.1589).

Wanberg, Kammeyer-Mueller, and Marchese (2006), suggested that formal mentoring is an organized program that pairs participants (mentor/protégé) as part of an employee development practice; moreover, they advocated that formal mentoring relationships are “arranged for a specified duration (e.g. nine months to a year), and protégés are generally prompted to have developmental goals in mind" (p.411). According to Murray (1991), Zey (1985), and Eby and Lockwood (2004), formal mentorships often have contracted goals and a specific timeline. Ragins, Cotton and Miller (2000) suggested the assignment of participant dyads usually occurs through the organization (i.e. a third party), and in some cases "the mentor and protégé have not even met before the match is made (Murray, 1991)" (p.1179). 
Eddy, Tannenbaum, Alliger, D'Abate, and Givens, (2001) postulated that goals of formal mentoring include talent development, improvement of employee knowledge, skills, and abilities, employee retention, and diversity enhancements. Furthermore, McKimm, Jolie and Hatter $(1999,2003,2007)$ suggested formal mentoring programs are utilized by organizations for a multiplicity of motives which may include the following (Jones \& Jowett, 1997):

to identify potential more effectively; to induct new staff more quickly; to improve the retention of staff; to encourage and support high flyers; to encourage and support ethnic minority and disadvantaged groups; to encourage and support women to break through the glass ceiling; to support selfdevelopment and work-based learning programs, continuous professional development, graduate or company training programs; to support organizational change; to encourage personal development; to help individuals cope with transitions such as moving into a new job or role" (p.2).

According to Raabe and Beehr (2003), "the use of formal mentoring programs is likely to increase (Tyler, 1998) because of presumed positive impact on the [protégé]" (p.271).

\section{Mentoring and Engineering}

In engineering, according to Russell and Nelson (2009), "once the new graduate engineer is on the job, mentoring is the method most often applied to help develop young professionals" (p.40). However, Russell and Nelson (2009) do not believe that mentoring is being utilized adequately by engineering organizations in order to nurture the next generation of engineers. Russell (2006) believed "mentoring and its time-honored approach of one-on-one advice and consultation can broaden the reach, effectiveness, and appeal of engineering" (p.34). 
Furthermore, Russell (2006) stated that the effective use of mentoring in engineering organizations adds to the organization's' essence and momentum. Russell (2006) posited that in mentoring a young professional, it is important for engineering organizations to provide opportunities for the young professional (i.e., protégé) who is full of energy and new ideas, to take part in team building, brainstorming and planning sessions, as they have a thirst for information and a willingness to learn; moreover, it is imperative for the young professional to build relationships throughout the organization as mentoring is a two-way street, and the learning experiences and opportunities are of significant benefit to the mentor as well.

According to DeLong, Gabarro and Lees (2008), "professional service firms, [like oilfield engineering service firms] live and die by their intellectual capital" (p.121). Russell (2006) stated that "mentoring is perhaps the best way to ensure that one's intellectual and experiential legacy can be tapped, improved upon, and be of continual benefit to the organization" (p.35). "If [organizations] fail to nurture this talent, [they] will lose the heart and soul of [their] firm, as well as the very people [they] recruited to give [them] an edge in a hypercompetitive world" (DeLong, Gabarro \& Lees, 2008, p.121). Russell (2006) suggested engineering organizations and engineering service firms must revive formal mentoring programs, as engineering professionals need continuous learning opportunities commencing with their formal collegiate education and continuing with organizational initiatives through HRD programs, like mentoring.

Locurcio and Mitvalsky (2002) suggested that "mentoring should be divided into two phases: an orientation phase, which occurs when the young professional joins the firm, and the development and retention phase, which occurs when the person is seeking to achieve his or her career goals through personal development" (p.31). DeLong, Gabarro and Lees (2008) posited that young professionals want tangible, pro-active advice from a senior professional who has a 
personal interest in the young professional's career. Moreover, DeLong, Gabarro and Lees (2008) stated that organizations and their leaders must listen, inquire, and show interest in young professionals; moreover, the senior professionals (i.e. mentors), need to "ask an associate what kind of work s/he wants to do, where he/r passions lie, [and] what skills s/he wants to develop" (p.118).

Russell (2006) stated that "an official mentoring program can help ensure that new professionals become involved in the workplace - and become productive - as quickly as possible; however, a formal mentoring program will ensure that they [young professionals] are fully integrated into the company in a shorter period of time than if there were no such program" (p.31). According to DeLong, Gabarro and Lees (2008), all the professionals, be they young or seasoned, in an organization need individualized and customized mentoring, especially when assignment-based learning opportunities are limited; yet, most organizations only mentor those employees who offer the greatest return. Organizations should note that a homogenous mentoring system will not solve all problems; however, according to Russell (2006), "mentoring and its time-honored approach of one-on-one advice and consultation, can broaden the reach, effectiveness, and appeal of engineering" (p.34).

\section{LEAD Mentoring Program}

The LEAD (Learn, Excel, Achieve, Develop) Program is an in-depth training program including both theoretical and hands-on learning. The LEAD Program assigns each newly hired engineer a mentor that not only supports and directs the mentee through program curriculum, but also works closely with the mentee's manager and training personnel to ensure a comprehensive learning experience. One of the important components of LEAD is the Engineering Development Program (EDP). EDP comprises three distinct courses - EDP1, EDP2, and EDP3. 
EDP1 is a two-week course informing new hires about the organization as well as an overall understanding of the oil and gas industry. EDP1 is started within a few months of initial employment. EDP2 and EDP3 are five-day courses intended to develop leadership and management skills among new hires. These courses focus on learning different product line areas and allow new hires the opportunity to build a network of expert contacts.

Throughout the training period, each employee must complete the sequence of courses, structured on-job tasks, and activities designed to develop competency - from the initial understanding of the basic technology to the advanced knowledge and skill required to deliver effective services, the learning opportunities keep pace with the rapid increase in an employees' job responsibilities. Each mentor supports and directs the mentee through the program curriculum, assists the mentee in preparing for yearly assessments and final qualification and, ultimately, enables the mentee to become a mentor to less experienced engineers

\section{Mentoring Benefits: Perceived Organizational Support}

According to Raabe and Beehr (2003), mentoring is often described as consisting of three behaviors making up relationships between mentors and mentees: career development, social support, and role modeling. Researchers have suggested that mentors help mentees in at least two domains - social (e.g., Thomas, 1993) and career (e.g., Fagenson, 1989; Kram, 1985) and thus should make the employees more valuable to the organization as well as helping the employees' careers" (p.272). Rosser (2004) suggested that the career development functions emerge first, "and as the interpersonal bond strengthens with time, the psychosocial functions emerge" (p.24). "Kammeyer-Mueller and Judge (2008), theorized that "career functions include actions such as providing the protégés with human capital enhancement opportunities and links to powerful individuals in the organization, [and] psychosocial functions include counseling the 
protégé about anxieties and uncertainty, providing friendship and acceptance, and role modeling” (p.270). Rosser (2004) stated that career and psychosocial functions are "determined based on the needs of the protégé and by the roles the mentor can provide" (p.45).

\section{Career Oriented Functions}

Turban and Lee (2007), posited that "career functions enhance the likelihood of the junior colleague becoming successful" (p.23). Moreover, Wanberg, et al. (2003), suggested that career-oriented functions are designed to benefit the organization as well as the individual's career. In fact, career functions are particularly important to the protégés' future experiences and serve to assist the protégé in the progression through the organizational hierarchy.

Kram (1985) believed that career functions occurred due to the mentor's experience, organizational position, and influence within the organizational. "It is this structural role relationship that enables [the mentor] to provide sponsorship, coaching, and exposure-andvisibility to help a junior colleague [protégé] navigate effectively in the organizational world" (Kram, 1985, p.23).

Sponsorship is the most recurrently perceived career function, according to the literature. Kram (1985) posited that "during the early stage of an organizational career, sponsorship helps a newcomer build a reputation, become known, and obtain job opportunities that prepare him or her for higher level positions" (p.26). Sponsorship involves the mentor recommending and/or nominating the protégé for desirable advancement in the organization. It is suggested that "without sponsorship, an individual is likely to be overlooked for promotions regardless of his or her competence and performance" (Kram, 1985, p.25). "Sponsorship means actively helping the individual to get job experiences and promotions" (Ozkalp, Kirel, Sungur \& Ozdemir, 2008, p. 68). Kram proposed "the sponsorship function creates opportunities for advancement for the 
junior person [protégé], and, at the same time, it is a measure of the senior person's [mentor] credibility and organizational clout" (p.26-27).

Protégés learn how to effectively navigate the corporate world through coaching. Kram (1985) states that coaching "involves sharing a senior person's understanding of the important players - who can be trusted, who has the power, and who is likely to support or attack in a particular situation" (p.28-29). Coaching involves a critique and/or evaluation of a protégé's employment efforts and/or working style. "Coaching involves providing advice in both career and job performance" (Ozkalp, Kirel, Sungur \& Ozdemir, 2008, p. 68). Kram proposed "like sponsorship, successful coaching gains respect from one's peers and superiors" (p.29).

Protection is a career function that buffers or guards the protégé from possibly destructive contact with members of the organization that can damage the protégés reputation. Ozkalp, Kirel, Sungur and Ozdemir (2008), posited that protection means "shielding the mentee from potentially damaging experiences provides protection" (p. 68). "Protection involves taking credit and blame in controversial situations, as well as intervening in situations where the junior colleague is ill-equipped to achieve satisfactory resolution" (Kram, 1985, p. 29). Kram (1985) suggested protection either provides support or it can suffocate a protégé. Protection helps foster career advancement by "reducing unnecessary risks that can threaten an emerging reputation as a potential manager; it smothers the individual when it prevents exposure-and-visibility in high risk situations that can enhance the junior manager's [protégé's] reputation" (Kram, 1985, p. 30).

Lastly, the career function of exposure-and-visibility creates an opportunity for the protégé to associate and develop relationships with prominent department heads in the organization who authorize the further advancement of the protégé. "Facilitating exposure and visibility means providing opportunities for the mentee to develop relationships with key figures in the organization in order to advance" (Ozkalp, Kirel, Sungur \& Ozdemir, 2008, p.68). 
According to Kram (1985), exposure-and-visibility helps prepare protégés for "positions of greater responsibility and authority"; moreover it familiarizes the protégé with organizational decision-makers, thus making the protégé "a viable candidate" (p.27). "Kram (1985) suggested that "the exposure-and-visibility function not only makes an individual visible to others who may influence his organizational fate, but it also exposes the individual to future opportunities" (p.27).

\section{Psychosocial Functions}

Wanberg, et al (2003) suggested that psychosocial functions "are more personal, relying on an emotional bond between the mentor and protégé" (p.411). Turban and Lee (2007), posited that "psychosocial functions enhance the junior colleague's sense of competence and professional identity" (p.23). Lankau and Scandura (2007), believed that through psychosocial functions, "protégés increase their sense of competence, effectiveness, and self-worth" (p.98). Rosser (2004) utilized Kram's 1988 ideals regarding psychosocial functions and suggested that via psychosocial functions, protégés augment their sense of competency, cultivate their identity, and endorse their value in their employment role. Ragins and Cotton (1991) posited that psychosocial functions enhance a protégé's sense of ability, character, and effectiveness in a role.

Counseling is a psychosocial function that allows protégés to discover and share personal issues that could interfere and/or inhibit the protégé's self-esteem within the organization. Counseling allows and encourages the protégé "to talk openly about anxieties and fears" (Ozkalp, Kirel, Sungur \& Ozdemir, 2008, p.68); thus coping with personal concerns more effectively (Kram, 1985, p.36). Kram (1985) posited that "the more experienced senior colleague [mentor] provides a sounding board for this self-exploration, offers personal experience as an 
alternative perspective, and helps resolve problems through feedback and active listening” ( $\mathrm{p}$. 36).

Role modeling is the most frequently reported psychosocial function, as reported by literature. Kram (1985) and Ozkalp, Kirel, Sungur and Ozdemir (2008) suggested that the mentor's attitudes, values, and behavior provide an example for the mentor to imitate, i.e. role model. "Role modeling involves the senior person [mentor] setting a desirable example, and the junior person [protégé] identifying with it” (Kram, 1985, p.33). According to Kram (1985), role modeling is both a "conscious and unconscious process"; moreover, it "succeeds due to the emotional attachment formed" between the mentor and the protégé (p.33).

Friendship is the most informal psychosocial function. In fact, Ozkalp, Kirel, Sungur and Ozdemir (2008) defined friendship as "interaction informally with the mentee [protégé] at work" (p.68). Kram (1985) furthered this idea with the notion that the friendship function is

"characterized by social interaction that results in mutual liking and understanding and enjoyable informal exchanges about work and outside work experiences" (p.38).

The last and perhaps, most beneficial psychosocial function to the protégé is acceptanceand-confirmation, as both the mentor and the protégé develop their self-worth (in regards to their relationship) from the positive esteem exchanged. Ozkalp, Kirel, Sungur and Ozdemir (2008) defined acceptance and confirmation as the "conveying unconditional positive regard" (p.68). Kram (1985) believed that as the protégé developed and became more competent in the organization, the mentor's acceptance-and-confirmation provide the protégé with much-needed reinforcement and encouragement. Kram (1985) suggested that the acceptance-and-confirmation allows the protégé to experience independence and individuality. Without this function, the protégé is more likely to conform, and "in such instances, the protégé spends more energy trying to please and win acceptance and less energy exploring who s/he wants to become in the 
organizational world" (p.35). Ozkalp, Kirel, Sungur and Ozdemir (2008) suggested that all of "these functions enhance the protégés' sense of competence, identity, and work role effectiveness" (p.68). Bouquillion, Sosik, and Lee (2005) posited crucial requirements of psychosocial support are trust and commitment in the mentor/protégé relationship.

According to Parise and Forret (2007), there are numerous benefits derived from mentoring for both the protégé and the mentor. First, the personal satisfaction mentors receive from observing and participating in the success of their protégés (Allen et al., 1997; Eby \& Lockwood, 2005; Kram, 1985) can result in satisfying experiences for the mentor and reinforce their sense of competence and feelings of accomplishment (Kram \& Hall, 1989). Johnson (2007), posited that mentors can heighten protégé self-confidence or self-esteem. Ramaswami and Dreher (2007), suggested that by aiding protégés via mentoring, protégés create attainable goals and realize significant outcomes, thus, mentoring may enhance employee motivation. Moreover, Bearman, Blake-Bear, Hunt, and Crosby (2007), proposed that protégés stay attentive to relevant tasks as mentors help steer them away from unnecessary activities.

While protégés receive the guidance and assistance in the organization, protégés help mentors' job performance via fresh, new perspectives and perhaps, more technical innovate knowledge (Eby \& Lockwood, 2005; Kram \& Hall, 1989; Mullen \& Noe, 1999). Lastly, mentoring relationship may promote career success. Mentors can impart specific knowledge and expertise which contributes to protégé learning and skill development (Kram, 1985). By providing influential support as well as challenging assignments, protégés benefit from the mentor's sponsorship and protection (Gibb \& Megginson, 1993; Noe, 1988)."

Third, Allen and Eby, (2003) posited that individuals possess a common and fundamental "need to belong" and as such, this need may be an important driver of affective, cognitive, and behavioral outcomes for protégés. Protégés can become trusted allies (Kram \& 
Hall, 1996; Lankau \& Scandura, 2002) and form a trustworthy base of support for their mentors, particularly as the protégés advance in the organization. By introducing protégés to significant individuals within organizational situations, mentors facilitate professional networking (Kram, 1985). "These important career contacts can in turn lead to career success in terms of salary, promotions, and job offers" (Allen and Eby, 2007, p.256).

Fourth, if a protégé is developed into an imperative individual within the organization, the mentor may gain credit among peers and superiors for the protégé's development (Allen et al., 1997; Kram, 1985; Ragins \& Scandura, 1999). "Zey (1984) outlined the following benefits: (a) career enhancement for the mentor, who can build a reputation of effectively developing talent; (b) access to a communication conduit since the protégé can gather information and lobby on behalf of the mentor among other departments; and (c) intrapsychic rewards from making a contribution to the protégé as an individual and to the organization as a whole" (a.c. Ensher, \& Murphy, 1997, p. 461). Fifth, according to Godshalk and Sosik (2007), when protégés'succeed within the organization, the mentor may gain prestige or experience feelings of generativity or immortality (Kram, 1985; Ragins \& Scandura, 1999).

According to Baumeister and Leary (1995), mentoring is significantly associated with a variety of positive protégé and mentor outcomes, which is consistent with conventional understanding that close relationships are important for individuals regardless of their role. According to Egan and Song (2008), "formal mentoring may bring significant benefits to participants: protégés of the mentoring program cannot only benefit psychologically, including increased job satisfaction, higher commitment to their organization and perceived better fit with their organization, but also demonstrate higher job performance immediately after the program" (p.358-359). According to Ehrich, Hansford, and Tennent (2001), "networking, career satisfaction, improved skills, increased pride and assistance were the most frequently cited 
benefits for mentors and career satisfaction, coaching ideas, challenging assignments, and access to resources were the most frequently cited benefits for protégés" (p.12). Seibert (1999) found formally mentored employees reported greater job satisfaction than non-mentored employees.

Positive outcomes for the mentor and protégé also create positive outcomes for the organization. According to Ehrich, Hansford and Tennent (2001), "Positive outcomes for the organization in business settings included improved productivity, retention of staff, promotion of loyalty, an improved workplace, and that mentoring facilitates change" (p.12). In fact, Ensher and Murphy (1997) suggested that "in addition to the benefits received by the protégé, the mentor and the organization derive positive outcomes from the mentoring relationship" (Halatin, 1981; Hunt \& Michael, 1983; Zey, 1984; p.461).

According to Raabe and Beehr (2003), positive outcomes for employees included career self-reliance, enhanced career development, and individual career growth; whereas positive outcomes for the organization "included retention of valued employees, increased productivity, and acceleration in employee development" (p.277). According to Ensher and Murphy (1997), "benefits that are said to accrue to the organization include: (a) the integration or socialization of individuals into the operating norms and informal power structure; (b) increased organizational communication as mentors and protégé's form alliances across levels and departments; (c) management development and succession planning information; and (d) increased productivity and decreased turnover" (Wilson \& Elman, 1990; Zey, 1984; p.461). "It is important to mention the findings of Hegstad and Wentling (2004) and Egan and Rosser (2004), which established that companies enjoy improved performance, increased motivation, higher job satisfaction, and increased organizational commitment as benefits of mentoring relationships" (Rosser, 2004, p.50). 
Affective Reaction: Job Satisfaction

"Job satisfaction is one of the most widely researched yet least understood phenomena in organizations today" (Rowden, 2002, p.407). According to Lankau and Scandura (2002), "Job satisfaction is an affective attitudinal response to the work environment" (Weiss, Dawis, England, \& Lofquist, 1967; p.781). Egan and Song (2008) used Cranny, Smith, and Stone's, (1992) definition of job satisfaction and defined job satisfaction "as an employee's affective reactions to a job based on comparing desired outcomes with actual outcomes (p.353)." Rowden described job satisfaction as "how people feel about their jobs and different aspects of their jobs" (2002, p.412).

In regards to organizational productivity, Wright and Davis (2003) believed job satisfaction has important implications on efficiency. "It is assumed that the benefits [job satisfaction] that employees receive from their organization influence the effort, skill, and creativity that employees are willing to provide their employer" (Wright and Davis, 2003, p.70). Researchers suggested satisfaction is a good predictor of employee behavior. Lam, Pine and Baum (2003) suggested that "Individuals are more likely to intend to perform a behavior if they have a positive attitude towards it" (p.163).

Job Satisfaction and Mentoring

According to Egan and Song (2008), "There appear to be relatively few studies on job satisfaction associated with mentoring. In their meta-analysis focusing on protégé outcomes, Allen et al. (2004) identified 10 mentoring studies that included job satisfaction as a dependent variable."(p.353), and Egan and Rosser (2004) suggested these studies identified by Allen et al. (2004) showed "positive relationships between mentoring and job satisfaction" (p. 228). Wang, Tolson, Chiang, and Huang (2010) suggested that although job satisfaction is and has been one 
of the most widely studied subjects over the last four decades of organizational research, they concurred with Rowden and Conine (2005) in that it is the "least understood phenomenon in organizations today" (215). According to Ragins, Cotton, and Miller (2000), "Existing theory predicts that effective mentoring should be associated with positive career and job attitudes" (Kram, 1985; p.1178).

Noe, Greenberger and Wang (2002) agreed with Ragins, Cotton, and Miller (2000) in that protégés in mentoring relationships had "greater job satisfaction, organizational commitment, satisfaction with opportunities for promotion, career commitment, organizationally-based self-esteem, perceptions of justice, and lower intentions to quit" (p.131). According to Egan and Rosser (2004), "individuals with mentors often have more positive subjective outcomes including career satisfaction, job satisfaction, career commitment, and intention to stay at their organization" (p. 229). According to Burlew (1991), "Mentored employees tend to be more satisfied with their jobs, get faster promotions, and make higher salaries (Farylo \& Paludi, 1985; Johnson, 1980; Olian, Carroll, Giannantonio, \& Feren, 1988; Roche, 1979; p.213)." Eby, Durley, Evans, and Ragins (2006) uncovered the protégé's perspective and found a positive relationship between mentoring received by protégés and affective reaction: job satisfaction (Allen, et.al, 2004) and organizational commitment (Aryee \& Chay, 1994)" (p.437).

Appelbaum, Ritchie, and Shapiro (1994) posited that "mentoring affects many aspects of organizational behavior including: leadership, organizational culture, job satisfaction and performance" (p.8). In fact, with support from their study, Egan and Rosser (2004) stated that "the conclusion that the positive effects of mentoring on protégé job satisfaction, organizational commitment and performance is likely a characteristic outcome of formal mentoring" (p.232). According to Wang, Tolson, Chiang, and Huang (2010), "Overall, job satisfaction is a reflection 
of 'an employee's attitudes of overall acceptance, contentment, and enjoyment in their work" (Lee-Kelley, Blackman, \& Hurst, 2007, 206; p.149-150). Hackman and Oldham (1980) suggested that employees who experienced relevance in their work displayed increased satisfaction and motivation as well as reduced turnover.

Furthermore, according to Bowling and Hammond (2008), "the potential situation and dispositional causes of job satisfaction had been examined as a potential cause of important work related behaviors, such as job performance, absenteeism, and turnover" (p.63). Researchers have found that job satisfaction is theoretically and empirically linked to several withdrawal behaviors (i.e. turnover intentions). According to Lok and Crawford (2003), "when employees are dissatisfied at work, they are less committed and will look for other opportunities to quit. If opportunities are unavailable, they may emotionally or mentally 'withdraw' from the organizations" (p.321). Bowling and Hammond (2008) concurred and suggested "that withdrawal behaviors are a strategy used by workers to avoid unpleasant or dissatisfying work" (p.67).

Ensher and Murphy (1997) "found that employees who received mentoring (a) experienced more promotions, (b) had higher incomes, and are more satisfied with their pay and benefits" (Dreher \& Ash, 1990; Fagenson, 1989; Turban \& Dougherty, 1994; p.461). According to Ragins, Cotton and Miller (2000), "Individuals in highly satisfying mentoring relationships reported more positive attitudes than non-mentored individuals; however, Ragins, Cotton and Miller (2000) theorized that "the presence of a mentor alone does not automatically lead to positive work outcomes; the outcomes may depend on the quality of the mentoring relationship." (p.1190). In fact, Egan and Rosser (2004) utilized Porter and Steers (1973) argument such that “employees anticipate that their jobs will provide a blend of aspects (e.g., pay, promotion, or autonomy) to which the employee assigns preferences. The ranking of these preferences differ 
across individuals, but if expectations go unmet for an extended period, satisfaction is diminished and the probability for withdrawal behavior increases (Pearson, 1991)" (p.228).

\section{Affective Reaction: Organizational Commitment}

Organizational commitment has long been a concern of organizations, and therefore of researchers who examine industry human-resources concerns. According to Egan and Song (2008), "organizational commitment has often been framed as the psychological bond that ties the employee to his/her organization. Organizational commitment represents a positive feeling of congruent identity with one's organization that may include a sense of attachment or affiliation (Meyer \& Allen, 1997; p.353)." According to Donaldson, Ensher, and Grant-Vallone (2000), "organizational commitment refers to the strength of an individual's identification with, and involvement in, a particular organization" (Porter, Steers, Mowday \& Boulian, 1974; p. 235).

Meyer and Allen (1997) defined employee organizational commitment as the symbiotic process in which the goals and the attitude of the organization converge with the goals and the identity of the employee such that the employee perceives rewards connected with continued involvement with the organization. Mowday, Steers and Porter (1979) characterized organizational commitment as related to three factors: "(1) a strong belief in and acceptance of the organization's goals and values; (2) a willingness to exert considerable effort on behalf of the organization; and (3) a strong desire to maintain membership in the organization" (p.226). Wayne, Shore, and Liden (1993) suggested a pattern of reciprocity develops over time between an employee and their employing organization. Those employees who perceive low support may be more prone to leave the organization. In fact, Lam, Zhang, and Baum (2001) advocated, "most of the literature on employee turnover suggests that labor turnover is a "hidden" cost for 
most organizations. Additional recruitment and training costs must be incurred as well as a resulting decrease in productivity" (Lam, Zhang, \& Baum, 2001, p.157).

Most organizations underestimate the costs associated with turnover. Ward and Davis (1995) postulated that organization commitment deserves management attention as the concerns of low commitment can be costly to an organization. Hsu, Jiang, Klein, and Tang, (2002) suggested that "HR managers have tried numerous approaches to reduce the turnover rate. They generally focus on methods related to pay structure, including higher than average salaries and staying bonuses” (Hsu, Jiang, Klein, \& Tang, 2002, p.361). Simmons and Hinkin (2001) believed companies may systematically survey employee attitudes and reward managers for the extent to which they can foster a satisfied and committed workforce. Yet rewarding management does not solve the increasing rate of employee turnover within the oil-field services industry. Allen et al. (2000) discovered "most organizations probably believe their reward systems are relatively fair; however, many employees would not agree” (p.103). Moreover, Hartmann and Bambacas (2000) suggested that "many organizations are perfectly willing to accept low commitment as a consequence of low salary and overhead expenses" (p.90).

The influence of human resource development programs on the turnover process has important implications for organizations; however, Allen, Poteet, and Russell (2000) reminded managers that not all of the implemented human resource practices will directly affect turnover. Hinkin and Tracey (2000) discovered that turnover is more likely to be associated with current job dissatisfaction rather than from an attraction to other job opportunities. In fact, employee commitment and job satisfaction are found to be negatively related to intentions to quit (Allen, et al., 2000).

“Turnover is a symptom of underlying problems... caused primarily by poor supervision, a poor work environment, and inadequate compensation" (Hinkin \& Tracey, 2000, 
p.21). Gouillart and Kelly (1995) suggested that organizations with environments that encourage self-development may increase organizational commitment, such that an individuals' desire to seek employment elsewhere ceases, especially if the employee is acquiring new skills and competencies that allow them to increase their self-efficacy. According to Mattei (2001), "In the opinion of Russell Campanello, an executive with Nervewire, Inc., "The number one reason why people leave their jobs is to pursue development - the chance to learn something new" (p.44). Locurcio and Mitvalsky (2002) concurred and suggested that "it is important that young professionals feel they are an integral part of the company's success and that the company is committed to the realization of their professional goals" (p.33).

The three predominant foundations in the organizational commitment literature, according to Egan and Rosser (2004) are "a moral obligation to remain with the organization, commitment reflecting an affective identification with the organization, and recognition of costs associated with leaving the organization" (p.229). According to Mowday, Steers and Porter (1979), commitment is a key component in understanding employee work behavior. Meyer, Allen, and Gellatly (1990) delineated turnover and organizational commitment from an intent to leave "as intentions about leaving indicate a response to the employee's current situation, which is not necessarily tempered by the reality of felt obligation, opportunities and costs (Hartmann \& Bambacas, 2000, p.95). Hartmann and Bambacas (2000) suggested that regardless of the type of commitment an employee may feel, and or even the employees' satisfaction with the organization, "some employees continually think about leaving an organization and promoting their careers" (p.95-96).

Researchers suggested several different aspects of commitment - attitudinal, affective, continuance, and normative. According to Sheldon (1971), attitudinal commitment exists when 'the identity of the person (is linked) to the organization" (p. 143) and Hall, Schneider, and 
Nygren. (1970) suggested that attitudinal commitment is present when 'the goals of the organization and those of the individual become increasingly integrated or congruent" (p. 176). Mowday, Steers and Porter (1979) further reflected that "attitudinal commitment thus represents a state in which an individual identifies with a particular organization and its goals and wishes to maintain membership in order to facilitate these goals" (p.225).

Affective commitment, according to Mowday, Steers and Porter (1979), refers to feelings of belonging and an attachment, or connection to the organization. Affective commitment is linked to individual characteristics in relation to organizational structures and work experiences, namely, compensation, management, role clarity, and skill variety. Continuance commitment, according to Mowday, Steers and Porter (1979), relates to supposed costs of separation, both financial and non-financial as well as an alleged absence of employment alternatives.

Normative commitment, according to Mowday, Steers and Porter (1979), is “concerned with the obligation employees feel to remain with an organization and builds upon what Wiener (1982) described as generalized cultural expectations that "a man" should not change his job too often or "he" may be labeled untrustworthy and erratic" (p.93). Meyer and Allen (1997) posited that an employee's "intent to remain or quit the organization is one important consequence that is expected to vary with each aspect of commitment and has been consistently correlated with affective and normative commitment but slightly less so to continuance commitment" (Hartmann \& Bambacas, 2000, p.95).

According to Mowday, Steers, and Porter (1979), “day-to-day events in the work place may affect an employee's level of job satisfaction; [however], such transitory events should not cause an employee to seriously reevaluate his or her attachment to the overall organization" (p.226). As such, organizational commitment is a widely recognized construct for predicting 
employee workplace performance and organizational success (Bateman \& Strasser, 1984; Brooks, 2002; Samad, 2005, 2006).

\section{Organizational Commitment and Mentoring}

Researchers elucidated that an individuals involvement in mentoring, albeit the protégé or the mentor, positively correlates with organizational commitment. Heimann \& Pittenger (1996) established that in formal mentoring programs, mentoring effects on protégé' organizational commitment were positive. Ragins and Cotton $(1999 ; 2000)$ found evidence of increased organizational commitment associated with mentoring. Furthermore, according to results from Egan and Song's (2008) study, Egan and Song concluded "that the positive effects of mentoring on protégé's job satisfaction and organizational commitment is likely a characteristic outcome of formal mentoring" (p.358).

Noe, Greenberger, and Wang (2002) posited "mentoring is also associated with lower turnover intentions (Scandura \& Viator, 1994) and decreased work alienation (Koberg, Boss, Chappell \& Ringer, 1994)" (p.130). Turban and Lee (2007), concurred with Allen et al. (2004), and stated that "mentored versus non-mentored individuals reported higher compensation, more promotions and greater career satisfaction, career commitment, and job satisfaction" (p.21). Eby, et al. (2006), found "immediate proximal benefits reported by mentors were positively associated with both their job satisfaction and their organizational commitment” (p.437). Allen's 2007 findings coincided with Lentz and Allen (2005) and state "that those with experience as mentors reported greater job satisfaction, greater affective organizational commitment, and fewer intentions to turnover than did those with no experience as mentors.

Some researchers dictated the level of commitment is based upon the quality of the mentoring relationship. For instance, Donaldson, Ensher, and Grant-Vallone, (2000) posited high 
quality mentoring relationship correlate with organizational commitment; in fact, "the quality of one's mentoring relationship, in addition to whether or not a protégé has a mentor (e.g. Chao G. , 1997), can have immediate and somewhat longer term effects on important organizational behaviors such as organizational commitment" (p.243).

\section{Affective Reaction: Job Satisfaction and Organizational Commitment}

Researchers suggested that job satisfaction yields positive relationships with other job attitudes, such as organizational commitment (Mathieu \& Zajac, 1990; Meyer, Stanley, Herscovitch, \& Topolnytsky, 2002). According to Wang, Tolson, Chiang, and Huang (2010), "Researchers have consistently reported a positive relationship between workplace learning and job satisfaction (Rowden \& Conine, Jr., 2005; Schmidt, 2007) and between job satisfaction and organizational commitment (Balfour \& Wechsler, 1990; 1991; McFarlin \& Sweeney, 1992; Mowday, Steers \& Porter, 1979)" (p.150). Moreover, Iverson and Roy (1994) posited that overall job satisfaction leads to higher employee commitment to organizations.

Organizational commitment varied from the concept of job satisfaction in numerous constructs. Mowday, Steers and Porter (1979), suggested "commitment as a construct is more global, reflecting a general affective response to the organization as a whole. Job satisfaction, on the other hand, reflects one's response either to one's job or to certain aspects of one's job" (p.226). As such, organizational commitment highlights an attachment the employee feels to the employing organization, while job satisfaction encompasses the employee's specific job and/or employment situation. In fact, researchers seek to associate job satisfaction with commitment. According to Agho, Mueller, \& Price (1993), "Some who study job satisfaction focus on its influence on employee commitment, absenteeism, intentions to quit, and actual turnover" (ac. Egan and Rosser, 2004, p.228). 
Lok and Crawford (2003), stated that "organizational commitment and job satisfaction are important attitudes in assessing employees' intention to quit and the overall contribution of the employee to the organization" (p.321-322). Wang, Tolson, Chiang, and Huang (2010), suggested that even though correlations have been found among job satisfaction and organizational commitment, the investigation of these constructs is still in infancy.

\section{Summary}

The intention of this literature review was to give an in-depth assessment of the literature and the theoretical foundations that support cultural differences, organizational support (i.e., formal mentoring relationships), and affective reaction. A review of culture provided an understanding of how diversity and culture impact perceived organizational support (i.e., formal mentoring relationships). Hofstede's seminal work detailed the different cultural factors affecting organizations and their HR practices. The current review of literature provided a foundation to the current study of understanding the extent to which cultural values in an oilfield services corporation differ among employees. The current review of literature also detailed organizational support as defined by Kram (1985) - career and psychosocial support - within the oilfield services industry.

Kram's seminal work detailed mentoring functions, which explain how mentoring relationships in terms of career and psychosocial functions benefit an organization, not to mention, the additional benefits of affective reaction. Job satisfaction and organizational commitment provide a context for how mentoring benefits all parties involved, including the organization. By understanding the foundations, a strong basis was set for the importance of formal mentoring in developing employees. 


\section{CHAPTER III}

\section{METHODOLOGY}

The methodology section includes a brief description of the study design, the population of the study, sample of the study and demographic composition, the procedures used for data collection, the instruments utilized to collect data, and finally the details of the methods and techniques used for analysis of the data.

\section{Study Design}

The purpose of this study was to address the research question and the supporting hypotheses about cultural differences and values that may affect job satisfaction, organizational commitment, and participation in formal mentoring programs of Field Engineers 1 within an oilfield services corporation. An electronic questionnaire was utilized to collect self-reported data. A pilot test was conducted with 18 respondents to test the clarity, simplicity, and accessibility of the questionnaire. The pilot test respondents were all Field Engineers 1 from one geographical location within the oilfield services organization. Cronbach's alpha was used to estimate reliability for the three factors of cultural values, perceived organizational support, and affective reaction. Principal Component Analysis (PCA) was conducted to analyze the factor structure of all the constructs involved in the study. A correlation coefficient (r) analysis was conducted to examine if the variables correlated with each other as hypothesized. A regression analysis, both linear and ordinal, was conducted to examine if the independent variable(s) in the study predicted the dependent variables as hypothesized. A path analysis was conducted to test the four models hypothesized in the study and to test the goodness of fit of the models. Mediation tests were conducted to examine if there were mediating effects. 


\section{Population}

The population of this study included 892 Field Engineers 1 (FE1s), globally, at an oilfield services corporation. Since the pilot test was conducted at one geographic location, the pilot test respondents were not included in the study sample.

\section{Study Sample}

Although 387 responded to the survey, the final sample size was 341, representing a population of 892 (from the total Field Engineers 1 (FE1s) who received the questionnaire; specific sampling details provided below). Forty-six respondents were removed because of either incomplete responses or they fell outside the range for the time employed in their position, which was any period greater than five (5) years. The respondents included Field Engineers 1 (FE1s) from both hemispheres, of the 341 respondents, 231 were from Eastern Hemisphere and 110 were from Western Hemisphere. The respondents were further separated into the following nine geographical locations by the oilfield services organization: Africa, Asia Pacific, Canada, Europe, Gulf of Mexico, Latin America, Middle East, Russia \& Caspian, and US Land. Due to conducting the Pilot Study with Canada specific respondents, Canada was removed from the final results. Due to only four responses from the Gulf of Mexico, the respondents from the Gulf of Mexico were also removed from the final results. Within the seven geographical locations (as set by the oilfield services organization) the following responses were received: 46 were from Africa, 70 were from Asia Pacific, 26 were from Europe, 52 were from Latin America, 50 were from Middle East, 39 were from Russia \& Caspian, and 58 were from US Land.

In order to further stratify the sample, the respondents were separated by country and responses were received from forty-two countries. A breakdown of responses per present country of citizenship is in Table 1 . 
Table 1 Number of Respondents per Present Country of Citizenship

\begin{tabular}{|c|c|c|c|}
\hline Country & $\begin{array}{c}\text { Usable } \\
\text { Responses }\end{array}$ & Country & $\begin{array}{c}\text { Usable } \\
\text { Responses }\end{array}$ \\
\hline Algeria & 3 & Kazakhstan & 7 \\
\hline Angola & 2 & Kuwait & 5 \\
\hline Australia & 21 & Malaysia & 13 \\
\hline Azerbaijan & 7 & Mexico & 16 \\
\hline Brazil & 20 & Norway & 5 \\
\hline Brunei & 5 & Oman & 6 \\
\hline Chad & 3 & Pakistan & 3 \\
\hline China & 10 & Peru & 1 \\
\hline Colombia & 12 & Qatar & 3 \\
\hline Congo & 11 & Romania & 3 \\
\hline Denmark & 1 & Russia & 24 \\
\hline Ecuador & 1 & Saudi Arabia & 9 \\
\hline Egypt & 12 & Spain & 1 \\
\hline Equatorial Guinea & 5 & Thailand & 4 \\
\hline Gabon & 9 & Turkmenistan & 1 \\
\hline Germany & 3 & Uganda & 1 \\
\hline Ghana & 12 & $\begin{array}{l}\text { United Arab } \\
\text { Emirates }\end{array}$ & 8 \\
\hline India & 5 & United Kingdom & 11 \\
\hline Indonesia & 7 & $\begin{array}{l}\text { United States of } \\
\text { America }\end{array}$ & 58 \\
\hline Iraq & 4 & Venezuela & 2 \\
\hline Italy & 2 & Vietnam & 5 \\
\hline
\end{tabular}

In terms of other demographics, number of years employed with the oilfield services corporation, as well as the number of years of experience in the current position. Of the 341 respondents, 37 had been employed with the oilfield services company for less than six (6) months. Of the 341 respondents, 140 had been employed for more than six (6) months but less than one (1) year. Of the 341 respondents, 109 of the respondents had been employed one (1) year to less than three (3) years. Of the 341 respondents, 38 of the respondents had been 
employed three (3) years to less than five (5) years. Of the 341 respondents, 3 of the respondents had been employed five (5) years to less than eight (8) years. Of the 341 respondents, 38 of the respondents had been employed eight (8) years to less than ten (10) years, and none of the respondents had been employed more than ten (10) years. The oilfield services organization insisted that Field Engineers 1 (FE1s) should only hold the FE1 position during the first years of employment; as such, respondents in the range period of over five years employed with the organization were removed from the respondent total. An analysis of variance (ANOVA) was conducted to determine if there were any differences among groups based on their years of experience on the job. The respondents were categorized into seven groups, $0-6$ months, 6 months-1 year, 1-3 years, 3-5 years, 5-8 years, 8-10 years, $10+$ years. The p-value for the obtained ANOVA are shown in Table 2.

Table 2 Summary of ANOVA for Cultural Indices across Years Employed

\begin{tabular}{|l|c|c|c|c|c|}
\hline & IDV & PDI & MAS & LTO & UAI \\
\hline Entire sample & 0.071 & 0.980 & 0.041 & 0.902 & 0.027 \\
\hline Eastern Hemisphere & 0.037 & 0.754 & 0.038 & 0.419 & 0.095 \\
\hline $\begin{array}{l}\text { Western } \\
\text { Hemisphere }\end{array}$ & 0.950 & 0.261 & 0.203 & 0.329 & 0.054 \\
\hline Africa & 0.996 & 0.059 & 0.423 & 0.936 & 0.394 \\
\hline Asia Pacific & 0.301 & 0.681 & 0.177 & 0.334 & 0.334 \\
\hline Europe & 0.315 & 0.526 & 0.607 & 0.385 & 0.631 \\
\hline Latin America & 0.978 & 0.189 & 0.789 & 0.539 & 0.026 \\
\hline
\end{tabular}


Table 2 continued.

\begin{tabular}{|l|c|c|c|c|c|}
\hline & IDV & PDI & MAS & LTO & UAI \\
\hline Middle East & 0.020 & 0.767 & 0.462 & 0.267 & 0.336 \\
\hline North America & 0.826 & 0.668 & 0.060 & 0.348 & 0.175 \\
\hline Russia \& Caspian & 0.616 & 0.798 & 0.465 & 0.951 & 0.489 \\
\hline
\end{tabular}

A further explanation for Table 2 can be found in Appendix 5.

In terms of the number of years of employed in the current position, of the 341

respondents, 58 had experience in the current position with the oilfield services company for less than six (6) months. Of the 341 respondents, 138 were employed in the current position for more than six (6) months but less than one (1) year. Of the 341 respondents, 111 of the respondents were employed in the current position one (1) year to less than three (3) years. Of the 341 respondents, 23 of the respondents were employed in the current position three (3) years to less than five (5) years. Eleven respondents did not specify time employed in current position. An analysis of variance (ANOVA) was conducted to determine if there were any differences among groups based on their years of experience on the job. The respondents were categorized into seven groups, 0-6months, 6 months-1 year, 1-3 years, 3-5 years, 5-8 years, 8-10 years, $10+$ years. The results of the ANOVA are shown in Table 3.

Table 3 Summary of ANOVA for Cultural Indices across Years Employed in Current Position

\begin{tabular}{|l|c|c|c|c|c|}
\hline & IDV & PDI & MAS & LTO & UAI \\
\hline Entire sample & 0.435 & 0.530 & 0.051 & 0.350 & 0.001 \\
\hline Eastern Hemisphere & 0.259 & 0.913 & 0.028 & 0.436 & 0.061 \\
\hline Western Hemisphere & 0.998 & 0.316 & 0.252 & 0.218 & 0.018 \\
\hline Africa & 0.611 & 0.593 & 0.824 & 0.984 & 0.781 \\
\hline Asia Pacific & 0.488 & 0.604 & 0.018 & 0.150 & 0.502 \\
\hline
\end{tabular}


Table 3 continued.

\begin{tabular}{|l|c|c|c|c|c|}
\hline & IDV & PDI & MAS & LTO & UAI \\
\hline Europe & 0.010 & 0.490 & 0.532 & 0.011 & 0.513 \\
\hline Latin America & 0.682 & 0.534 & 0.545 & 0.832 & 0.013 \\
\hline Middle East & 0.451 & 0.665 & 0.294 & 0.586 & 0.266 \\
\hline North America & 0.866 & 0.399 & 0.030 & 0.148 & 0.285 \\
\hline Russia \& Caspian & 0.559 & 0.999 & 0.870 & 0.981 & 0.007 \\
\hline
\end{tabular}

A further explanation for Table 3 can be found in Appendix 6.

In terms of gender, of the 341 respondents, 271 were male and 60 were female. The sample seems to be evenly distributed in terms of years, experience, and location. This apparent distribution was not tested as the sample sizes were too small when using the test criteria $n \pi \geq 10$ and $n(1-\pi) \geq 10$ where $n$ is the sample size and $\pi$ is the proportion.

\section{Procedure}

Electronic questionnaires using Qualtrics Survey Software were sent to collect data from the selected participants. The Human Resources Talent Management Director and the Human Resources Training Development Manager for Global Training were involved in the design process because of their familiarity with the sample. Both assisted the researcher with 'organization-specific' verbiage for the electronic questionnaire. An email with a cover letter embedded into the body of the email introduced the study and the researcher, and contained contact information of the researcher's advisor as well as contact information of the Institution Review Board at Texas A\&M University. Also included in the email, and in the questionnaire, were the ethical guidelines that would be followed by the researcher, which included the terms of consent and the assurance of confidentiality.

The electronic questionnaire thus had an introductory email that included the items mentioned above, as well as a log-in link that had instructions for entering the questionnaire, 
contact information of the researcher in case the respondent had problems accessing the questionnaire or had questions about the study, and information sheet, an informed consent page that contained a "Yes, I agree to participate" button that upon clicking, entered the respondent into the first set of questions, or allowed the respondent to exit, the thirty-five (35) item questionnaire, and the demographics page (see Appendix regarding the questionnaire and related correspondences).

The questionnaires were sent using an internal distribution link via Qualtrics Survey Software with the 'reply-to’ email address from the researcher (hannahayes@neo.tamu.edu). The Human Resources department personnel suggested that an 'outside of the organization' email address would be more beneficial and would aid in creating a less-biased response per the ability of the respondent's answers to remain confidential; moreover, their employees had been receiving questionnaires internally and hence, an internally emailed questionnaire might be overlooked. The e-mail subject heading was labeled Questionnaire to FE-1's on Mentoring thus detailing the chosen participants and the topic of the email questionnaire.

Considering that for a population of 900 , the recommended sample is 169 (Krejcie \& Morgan, 1970), this sample size well exceeded the required number of respondents for the population under study (total accessible population). The expected response rate (per the organizations standard internal questionnaire response rate) was forty percent (40\%). The researcher sent the first reminder email after a week of sending the questionnaire, and an additional follow up email one week later. The entire data collection process was completed between May 3, 2011 and May 23, 2011. The response rate was thirty-nine (39\%). The response rate was in-line with the average response rate garnered by the oilfield services organization when conducting their own questionnaires; however, the researcher had hoped for a greater response rate. The researcher suggested that the response rate could have been higher than the 
average rate generated via the organization's internal questionnaire method; however, in-line result was due to participant disinterest, lack of motivation to complete the twenty-minute questionnaire, and/or lack of participant time to complete the twenty-minute questionnaire. In fact, the researcher received emails from several participants on company-related jobs citing the lack of time due to hectic work schedules for their lack of ability to respond.

In order to handle non-response error, the researcher utilized Dooley and Linder's (2003) non-response error Method 4, thus comparing the respondents to the non-respondents based on characteristics. "Comparisons of respondents and non-respondents on characteristics known a priori [were] made to describe similarities and differences between respondents and nonrespondents" (Dooley \& Linder, 2003, p. 109). See Appendix A-11 for further explanation on respondents vs. non-respondents.

A reliability estimate for the total instrument and each of the item sets was conducted for the data. Cronbach's alpha reliability estimate for the entire instrument was 0.865 , mentoring (MFQ) 0.818 , job satisfaction (OJSS) 0.829; organizational commitment (OCQ) 0.693, and finally for cultural values 0.905 . A reliability estimate was also run for each cultural index: Power Distance (PDI) 0.515, Individualism (IDV) 0.924, Masculinity (MAS) 0.567, Uncertainty Avoidance (UAI) -0.098 [a negative covariance exists between the terms], and Long-Term Orientation (LTO) 0.917.

\section{Instrumentation}

The instrument used for the current study utilized four exiting surveys for measurement: mentoring functions (Castro \& Scandura, 2004), overall job satisfaction (Cammann, Fichman, Jenkins, \& Klesh 1983), organizational commitment (Marsden, Kalleberg, \& Cook, 1993), and cultural values (Hofstede, 1980, 1994). This instrument had 44 items and consists of five 
sections. The five sections comprised a collection of complete research instruments that have been utilized in other research studies. The instruments were chosen keeping two criteria in mind: (1) that the instruments met reasonable validity and reliability standards, and (2) that the instruments were short and practical to administer in terms of the amount of time to complete them. Except for Hofstede's Cultural Values instrument (which was 20 questions in length), and the additional mentoring questions, all other instruments met the above two criteria. The value of the additional mentoring questions had not been established.

The instrument used in this study was found to take less than twenty minutes to complete. Although reliability and validity of the four scales of measures, mentoring, job satisfaction, organizational commitment, and cultural values have been established in previous studies, the researcher estimated the reliability and explored cross-validation for all four scales using the current study's sample.

\section{Measuring Mentoring}

The Measure of Mentoring Functions, developed by Scandura (1992) contained an eighteen (18) item survey designed to assess vocational support, psychosocial support and role modeling. It can and has been used to assess the amount of mentoring in virtually every type of mentoring program; however, for the needs of this study, the researcher chose to use the reduced instrument created and validated by Castro and Scandura (2004). The Mentoring Functions Questionnaire (MFQ-9) measures nine (9) items and gives evidence supporting three constructs (vocational support, psychosocial support and role modeling, three items for each dimension) of its structure and the convergent and discriminant validity of MFQ scores (Castro \& Scandura, 2004). 
For purposes of this research, which so heavily focused on Kram's (1985) mentoring theoretical foundation findings of career support and psychosocial support, only the vocational support and psychosocial support factors were utilized, and as such, only six (6) items were measured. As such, section one of the questionnaire contained six (6) items from the Mentoring Functions Questionnaire (MFQ-9) created by Castro and Scandura (2004), which includes a scale from 1-7, with 1 being strongly disagree and 7 being strongly agree. Factor analysis was used to re-examine the construct validity of the items. Cronbach's alpha was used to estimate reliability using the current study's sample.

\section{Measuring Intent to Participate in Mentoring}

In addition to the MFQ, the oilfield services organization requested specific questions pertaining to the oilfield services formal mentoring program, including (1) if the respondent was currently involved in a mentoring program (M1), (2) if the respondent felt that the mentoring program was of benefit to the respondent, (3) if not involved in the mentoring program, if the respondent would be interested in participating in formal mentoring (M2), and (4) if the respondent had any concerns about being involved in the formal mentoring program. Factor analysis was used to examine the construct validity of the items. Cronbach's alpha was used to estimate reliability using the current study's sample.

\section{Measuring Job Satisfaction}

Section two of the questionnaire contained the three (3) item Overall Job Satisfaction Survey, as developed by Cammann, Fichman, Jenkins, and Klesh (1983). The Overall Job Satisfaction Survey used three-items to assess an "employee's subjective response to working in his or her job and organization" (Fields, 1983, p.5). Each item is measured using a 7-point Liker- 
type scale ranging from "strongly disagree" to "strongly agree." Items are written in both directions, with one reverse scored. According to Fields (1983), the "coefficient alpha values ranged from 0.67 to 0.95 (p.5). Factor analysis was used to re-examine the construct validity of the items. Cronbach's alpha was used to estimate reliability using the current study's sample.

\section{Measuring Organizational Commitment}

The third section of the questionnaire contained the six (6) item Organizational Commitment questionnaire, validated by Marsden, Kalleberg, and Cook (1993), which includes a scale from 1-4, with 1 being strongly disagree and 4 being strongly agree. The internal consistency of the instrument has been tested by Marsden, Kalleberg, and Cook (1993) and the coefficient $\alpha$ was 0.78 (a.c. Fields, 1983, p.56). According to Fields (1983), this questionnaire was "developed for and used in the 1991 General Social Survey", and meets the needs of largesample surveys (p.56). Factor analysis was used to re-examine the construct validity of the item. Cronbach's alpha was used to estimate reliability using the current study's sample.

\section{Measuring Cultural Values}

The fourth section of the questionnaire contained the twenty (20) item Values Survey Module 1994 (VSM 94) which includes a multitude of scales. Values Survey Module 1994 (VSM 94), is a questionnaire that was used in a study conducted by Hofstede and Hofstede in 1994. The Values Survey Module 1994 (VSM 94) was developed for comparing culturally influenced tenets of similar respondents from two or more regions within countries. It allows scores to be computed on five dimensions of national culture on the basis of four questions per dimension. Factor analysis was used to re-examine the construct validity of the items. Cronbach's alpha was used to estimate reliability using the current study's sample. 
The five dimensions measured (Hofstede, 2001; Hofstede \& Hofstede, 2005) focused on key issues in national societies, known from social anthropology and cross-cultural research. The earliest public version of the Values Survey Module (VSM 82) resulted from a comparison of subsidiaries of the IBM Corporation in 40 countries. Hofstede's research, and subsequent usage of his instrument, has shown that the answers to the 20 content questions are influenced by the nationality of the respondents. Hofstede and Hofstede, 1994 suggested that:

This is not to say that every respondent of nationality A gives one answer and every respondent of a nationality B another, but one can expect systematic differences between the average answer from a sample with nationality A and a comparable sample from nationality B (in statistical terms, an analysis of variance on the answer scores shows a significant country effect) (p.3, paragraph $3)$.

The content questions attributed to a dimension were selected because in comparisons of matched samples from ten or more countries, the mean country scores on the four questions belonging to the same dimension usually vary together (if one is high, the other is high, or low if it is a negatively formulated question; if one is low, the other is low, etc.). In statistical terms, the mean country scores are significantly correlated. The mean country scores on questions belonging to different dimensions usually do not vary together (are uncorrelated). Therefore, the 20 questions form 5 clusters of 4 questions each.

The twenty content questions allow index scores to be calculated on five dimensions of national value systems as components of national cultures: Power Distance (large vs. small), Individualism vs. Collectivism, Masculinity vs. Femininity, Uncertainty Avoidance (strong vs. weak), and Long- vs. Short-Term Orientation. All content questions are scored on five-point 
scales (1-2-3-4-5). Index scores are derived from the mean scores on the questions for national samples of respondents as explained below.

\section{Power Distance Index (PDI)}

Power Distance is defined as the extent to which the less powerful members of institutions and organizations within a society expect and accept that power is distributed unequally. The index formula is

$$
\text { PDI }=-35 m(03)+35 m(06)+25 m(14)-20 m(17)-20
$$

in which $\mathrm{m}(03)$ is the mean score for question 03 , etc. The index normally has a value between 0 (small Power Distance) and 100 (large Power Distance), but values below 0 and above 100 are technically possible.

\section{Individualism Index (IDV)}

Individualism is the opposite of Collectivism. Individualism stands for a society in which the ties between individuals are loose: a person is expected to look after himself or herself and his or her immediate family only. Collectivism stands for a society in which people from birth onwards are integrated into strong, cohesive in-groups, which continue to protect them throughout their lifetime in exchange for unquestioning loyalty. The index formula is

$$
\text { IDV }=-50 m(01)+30 m(02)+20 m(04)-25 m(08)+130
$$

in which $\mathrm{m}(01)$ is the mean score for question 01 , etc. The index normally has a value between 0 (strongly collectivist) and 100 (strongly individualist), but values below 0 and above 100 are technically possible. 


\section{Masculinity Index (MAS)}

Masculinity is the opposite of Femininity. Masculinity stands for a society in which emotional gender roles are clearly distinct: men are supposed to be assertive, tough, and focused on material success; women are supposed to be more modest, tender, and concerned with the

quality of life. Femininity stands for a society in which emotional gender roles overlap: both men and women are supposed to be modest, tender, and concerned with the quality of life. The index formula is

$$
\text { MAS }=+60 m(05)-20 m(07)+20 m(15)-70 m(20)+100
$$

in which $\mathrm{m}(05)$ is the mean score for question 05 , etc. The index normally has a value between 0 (strongly feminine) and 100 (strongly masculine), but values below 0 and above 100 are technically possible.

\section{Uncertainty Avoidance Index (UAI)}

Uncertainty Avoidance is defined as the extent to which the members of institutions and organizations within a society feel threatened by uncertain, unknown, ambiguous, or unstructured situations. The index formula is

$$
\mathrm{UAI}=+25 \mathrm{~m}(13)+20 \mathrm{~m}(16)-50 \mathrm{~m}(18)-15 \mathrm{~m}(19)+120
$$

in which $\mathrm{m}(13)$ is the mean score for question 13 , etc. The index normally has a value between 0 (weak Uncertainty Avoidance) and 100 (strong Uncertainty Avoidance), but values below 0 and above 100 are technically possible. 


\section{Long-term Orientation Index (LTO)}

Long-term Orientation is the opposite of Short-term Orientation. Long-term Orientation stands for a society that fosters virtues oriented towards future rewards, in particular perseverance and thrift. Short-term orientation stands for a society that fosters virtues related to the past and present, in particular respect for tradition, preservation of "face", and fulfilling social obligations. The index formula is

$$
\text { LTO }=-20 m(10)+20 m(12)+40
$$

in which $\mathrm{m}(10$ is the mean score for question 10 , etc. The index normally has a value between 0 (very short-term oriented) and 100 (very long-term oriented), but values below 0 and above 100 are technically possible.

\section{Data Analysis}

The data analyses included descriptive statistics, reliability estimation, factor analyses, correlation analysis, regression analysis, and path analysis using AMOS. The path analytic approach was utilized to examine the correlation between the variables in four hypothesized models. The details of the analyses and the statistical techniques utilized to analyze and report the data are described in the following sections. SPSS 16 was used for descriptive statistics, reliability analysis, factor analysis, correlation analysis, and regression analysis. AMOS was used to conduct the path analyses.

\section{Descriptive Statistics}

Descriptive statistics included the number of participants who took part in the survey, the range of scores, and the means, medians, modes, standard deviations, skewness, and respondent ratio for all the items. 
Reliability Analysis

According to Carmines and Zeller (1979), a "tendency towards consistency found in repeated measurements of the same phenomenon is referred to as reliability" (p. 12). Cronbach's alpha or alpha coefficient is the most often used technique in estimating internal-consistency reliability. In the current study, the reliability of the study as a whole as well as for the three factors: cultural values, perceived organizational support, and affective reaction, were estimated using Cronbach's alpha technique. The alpha coefficient for the intent to participate in formal mentoring was not determined as it consisted of yes/no questions.

\section{Factor Analysis}

Although the four instruments involved in the current study that focused on mentoring, cultural values, perceived organizational support, and affective reaction utilized item sets that have already been tested for validity, it was decided to explore cross-validation of the scales for all the variables utilizing the current study's sample. Factor analysis was used to verify the validity of the items. Factor analysis is a valuable tool used to study the internal structure of a set of items or indicators. This data reduction technique, according to Coolidge (2006), "reduces a set of variables to a smaller set by removing redundant variables" (p.365). Besides deriving a small set of variables from a large group of correlated variables, factor analysis also helps to identify underlying constructs.

Factor loading is an estimate of the validity of the items used to gauge a given construct because it signifies the relationship between each indicator and the factors being extracted. Factor loadings aid in determining which of the items correlated with the derived factor. The higher the factor loading, the greater the relationship of the indicator to the construct and the more valid the indicator or the item is with regards to the factor (Carmines \& Zeller, 1979). For 
the purpose of this study, it was decided to consider only factor loadings $\geq 0.3$. According to Tabachnick and Fidell (2001) the correlation (r), as found in the Correlation Matrix, should be .3 or higher in order to be considered in the same factor if the following two conditions are met: the Kaiser-Meyer-Olkin Measure of Sampling Adequacy (KMO) should be 0.6 or above and the Bartlett's Test of Sphericity should be significant (in this case 0.1 or smaller).The Principal Component Analyses (PCA) method was used for extraction of factors because, according to Voelz (2006), PCA is a data reduction technique useful for large, multi-dimensional data sets (p.1).

\section{Correlation Analysis}

In this study, the researcher used the correlation coefficient (r) to determine if there were positive or negative associations between the variables considered. Correlation analysis is utilized to examine if there is an association between two variables and/or whether there is an observed covariance between the two variables of interest (Coolidge, 2006). The range of the correlation coefficient or $r$ can be from -1 to +1 . While correlation coefficient of $r=+1$ suggests a perfect positive correlation, an $r=-1$ suggests a perfect negative correlation; an $r=0$ suggests that there is no relationship between the two variables of interest.

The correlation analysis was utilized, in many cases, in conjunction with the path analysis. This was done as a key requirement, normality, hadn't been met. Correlation was used to determine whether the relationship between various terms was positive or negative with the magnitude of that relationship left to the path analysis.

In this study the researcher hypothesized that there would be a significant correlation between perceived organizational support and affection. It is also hypothesized that there would be a significant positive correlation between perceived organizational support and affection and 
intent to participate in a formal mentoring program. All hypothesized relationships were unidirectional hence are defined as one-tailed.

\section{Regression Analysis}

Regression analysis was used in the current study to examine if the independent variables predicted the dependent variables. According to Coolidge (2006), "Regression analysis is a statistical procedure that measures the strength of a relationship between an independent and dependent variable" (p. 191). Regression analysis could also be dubbed prediction analysis as it measures the degree of the relationship between the predictor variable and the criterion variable. In this study the researcher hypothesized that cultural values would predict perceived organizational support. Similarly the researcher hypothesized that perceived organizational support and affective reaction would predict the intent to participate in formal mentoring programs. A correlation range of $\sim 0.3$ or above was used as the criterion to decide if the degree of prediction was significant. Later a utility of regression test was used to ensure that prediction was useful.

In this study, two methods were used accomplish the regression analysis: simple linear regression and path analysis. Two methods were used due the nature of the data; simple linear regression (discussed below) was not entirely applicable as the data was not normal; however, simple linear regression is commonly used. Initially, the requirements for the path analysis (discussed later) were also unmet, but the alternative requirements of Tabachnick and Fidell (2001) were met. In which case, the path coefficients found were applicable to the regression analysis. 


\section{Simple Linear Regression}

Simple linear regression is used to predict one (dependent) variable based upon one predictor. The relationship takes the following form:

$y=\beta_{o}+\beta_{1} x+\epsilon$ where $\mathrm{y}$ is the dependent variable, $\mathrm{x}$ is the predictor, $\beta_{o}$ is the $\mathrm{y}$ intercept, $\beta_{1}$ is the slope, and $\epsilon$ is the error.

Based on the sample data, $\beta_{1}$ is found by summing the square of the differences between the individual values of $\mathrm{x}$ and $\mathrm{y}$ while minimizing the error. $\beta_{o}$ is then found based on the linear form. Linear regression assumes that the following is true: 1) $\mathrm{x}$ and $\mathrm{y}$ are pairwise independent, 2) sample data is typical, and 3) the error is normal. Many relationships examined in this study were initially assumed to be linear.

The sum-of-squares (SS) is the sum of the square of the distance between the sample data points and the predicted line. This is done both horizontally (in the $\mathrm{x}$ direction) and vertically (in the y direction). The shortest distance between the observed data point and the predicted line is known as the residual or error (it is in the xy direction). Simple linear regression works by trying to minimize the sum-of-squares in terms of $\mathrm{x}, \mathrm{y}$, and $\mathrm{xy}$. It is expressed in the square of the units used for the Y values.

The value sy.x is the standard deviation of the vertical distances of the points from the line. Since the distances of the points from the line are called residuals, sy.x is the standard deviation of the residuals.

The value $\mathrm{r}^{2}$ quantifies goodness of fit. It is a fraction between 0.0 and 1.0, and has no units. Higher values indicate that the model fits the data better. A high $\mathrm{r}^{2}$ means that the line is very close to the observed values. That doesn't mean the fit is "good" in other ways. The best-fit values of the parameters may have values that make no sense (for example, negative rate constants) or the confidence intervals may be very wide. 
When $r^{2}$ equals 0.0 , the best-fit curve fits the data no better than a horizontal line going through the mean of all Y values. In this case, knowing X does not help you predict $Y$. When $r^{2}=1.0$, all points lie exactly on the line with no scatter; meaning that if $\mathrm{X}$ is known, then $\mathrm{Y}$ can be found.

$r^{2}$ is the fraction of the total variance of $Y$ that is explained by the model (equation). It is found using the sum of the squares of the distances of the points from the best-fit curve determined by linear regression. This sum-of-squares value is called Sy.x., which is in the units of the $\mathrm{Y}$-axis squared. To turn $\mathrm{r}^{2}$ into a fraction, the results are normalized using the sum of the square of the distances of the points from a horizontal line through the mean of all Y values.

\section{Utility of Regression}

Utility of regression is used to evaluate the predictive power of a regression, a linear regression in the case of this study. This is done by comparing the slope found during linear regression $\beta_{1}$ to a composite error term (both explainable and unexplainable). As long as this term falls within the range (i.e. the margin of error) of the expected value for the actual $\beta_{o}$ (for the population), regression is deemed useful.

\section{Ordinal Regression}

Ordinal regression is much like linear regression in concept. Rather than linking one predictor variable to one dependent variable, multiple predictor variables are linked to an ordinal dependent variable. For example, in this study, job satisfaction, consists of three variables to the ordinal result for intent to participate in formal mentoring (a yes/no response).

A resultant rank correlation $\left(\mathrm{R}^{2}\right)$ is then used to determine if there is a significant relationship. This rank correlation ranges from 0 (indicating that there is no relationship) to 1 
(indicating a perfect relationship). As this term is always positive, the results of the ordinal regression are often interpreted in combination with the results of either linear regression or a path analysis. This is done to determine whether the relationships were positive or negative. In some cases, a simple linear model was assumed to facilitate the collation of the differing resultants.

ANOVA

According to Tolson (2010), the ANOVA, the analysis of variance is a tool that "establishes the ratio of the differences between groups (means) to the differences within groups (error)" (p.135). Pallant (2005), suggested that ANOVA compares the "variance (variability in scores) between the different groups (believed to be due to the independent variable) within the variability with each of the groups (believed to be due to chance)" (p. 186). The comparison of one characteristic (e.g. years employed) yields a ratio (F) of the variability between the examined groups to chance. If this ratio, F, is large, then variability between groups is greater than that explained by chance, i.e. the groups appear to be the same. In which case, this ratio will be associated with a $p$ value which will be small compared to the study significance level. Use of the ANOVA test requires: a) the samples are independent, b) the standard deviations for all samples are homogeneous, and c) the samples are normal.

\section{Power of an ANOVA}

According to Zar (1999), the Power of ANOVA test indicates the probability that if an effect exists, it can be found. This test was not used in this study as normality requirements for the ANOVA had not been met. Additionally a negative covariance existed between Hofstede's 
UAI component variables; together this caused the Power to yield results that included the imaginary.

\section{MANOVA}

MANOVA is an extension of ANOVA, but rather than looking at one characteristic (e.g. years employed), more than one characteristic is examined (e.g. years employed and gender). These characteristics should be related or, as in the case of this study, have a hypothetical relationship. According to Pallant (2005), MANOVA compares groups in order to determine whether the mean differences between the combined groupings of the dependent variables are due to chance. A summary variable based upon the aspects being examined is created and is then compared to a linear combination of the aspects. This comparison takes the form of an ANOVA test. MANOVA can be used to specify if there is a significant difference between the constructed summary and the aspects both as a whole and individually.

While the MANOVA results could be accomplished using a series of ANOVA tests, it has the advantage of minimizing for a Type I error (concluding that the aspects are the same when they are actually different). In order to use MANOVA, the following guidelines must be met: 1) a minimum count of 3 for each aspect or a count of at least the number of aspects being examined; 2) multivariate normality - in practice this means a reasonably robust normality, which, according to Tabachnick and Fidell (2001), is accomplished by each variable having at least 20 counts. Otherwise this can be accomplished by checking univariate and multivariate normality and eliminating outliers; 3 ) the aspects being examined should be linearly related (i.e. a linear regression can be performed using them); 4) the correlation between the examined aspects should be low. Aspects with a high correlation should be removed. 
Multicollinearity is the undesirable situation in which the correlations between independent variables are strong. It increases the standard errors for the coefficients found during regression; coefficients found for independent variables may be found not to be significant, leading to erroneous conclusions.

Multicollinearity could lead to situations in which the model may fit the data well (such as a high factor loading during the Path Analysis), yet none of the $\mathrm{X}$ variables are statistically significant in explaining Y. This occurs when the variables convey essentially the same information. In SPSS, the multicolinearity for the model was be measured using the Variance Inflation Factor (VIF).

\section{Skewness}

Skewness describes how symmetric the normalized/standardized values (using the distributions mean and variance) are with regard to the mean. A negative skewness indicates that a negative bias is present, while a positive skewness indicates a positive bias is present. If the skewness is 0 , then there is no bias. Skewness can be determined by examining the distributions box plots. The skewness statistic is found from:

$\operatorname{skew}(X)=E\left(\frac{(X-E(X)}{\sqrt{\operatorname{Var}(X)}}\right)$ where $\mathrm{x}$ is the data point, $\mathrm{E}(\mathrm{)})$ is the expected value, and $\operatorname{Var}()$ is the variance.

\section{Welch Tests}

Welch's test (a variation of Student's t-test) is used with two samples having possibly unequal variances. Welch's Test is used when the data is non-normal and the variances are unequal. Welch's test, derived from Welch's 1938 and 1947 studies, is simply the un-pooled variances $t$-test also known as Satterwaite's test or the Welch-Satterthwaite test, after Satterwaite 
(1946), or the Smith/Welch/Satterwaite test, acknowledging the work from Smith, 1936 (Ruxton, 2006).

Path Analysis

A path analytic approach was used to depict the correlation matrices hypothesized in the study and to test the hypothesized causal paths between variables. Path Analysis is a subset of Structural Equation Modeling (SEM), a multivariate procedure which according to Ullman (1996), "allows examination of a set of relationships between one or more independent variables, either continuous or discrete, and one or more dependent variables, either continuous or discrete" (p.709). Unlike SEM, which considers both observed and unobserved variables, Path Analysis focuses on the observed variables. Unobserved variables are handled via the Maximum Likelihood estimate.

The Maximum Likelihood (ML) estimation method, in AMOS, was used to estimate the path coefficients and model fit. In ML, estimation of all the parameters in the model are computed at the same time and are iterative (estimates are repetitively calculated). Furthermore, in ML disturbances or error terms for the unobserved exogenous variables are accounted for. ML estimation is among the most widely used model-fitting estimation method. The AMOS program was used to test the path models because it includes the ML estimation method and also provides goodness of fit indices. Goodness of fit index is discussed later.

The paths for this study were hypothesized based on the results of the research garnered during the literature review, and the researchers' initial findings, suggesting a causal relationship between cultural values and perceived organizational support. A Structural Equation Model was used to depict the hypothesized relationships. A goodness of fit test was conducted between the hypothesized model/structural model and the identified model. 
Bartlett's Test

Bartlett's test is used to determine if the samples tested are from populations with equal variance. This is important to other tests, such as the ANOVA, as equal population variance is assumed. During the analysis, Bartlett's test in conjunction with the KMO were used satisfy the conditional requirements for the Path Analysis.

Kaiser-Meyer-Olkin Measure of Sampling Adequacy (KMO)

The Kaiser-Meyer-Olkin (KMO) is used to help determine the appropriateness of the sample tested for factor analysis. High values (between 0.5 and 1.0) indicate factor analysis is appropriate.

\section{Chi Square}

Chi-squared is used in one of two ways: for Goodness of Fit or Association. The chisquared test is non-parametric and has two requirements: 1) the expected or model frequencies are one or more, and 2) at most, $20 \%$ of the data has a frequency of less than 5 . In this study, the majority of the measurements were categorical in nature, making a non-parametric method necessary. The chi-squared distribution is a skewed distribution, like the ANOVA, so it can only dictate whether or not all aspects of the model or relationship match. If not, one or more aspects are different.

\section{Goodness of Fit}

According to Kaltenbach (2012), the Goodness of Fit (GIF) test is used to find out whether or not the study data is in agreement with the predicted values from a model. If they are in agreement, then the model could explain the study data. However, just because the model 
could explain the data doesn't mean that other models could not as well or better. This is important as the goodness of fit is used, in this study, to determine whether or not the models built during the path analysis are useful in explaining the study data.

\section{Ethical Considerations}

Institutional Review Board (IRB) rules and ethical regulations, as outlined in the Belmont Report, were followed. The researcher completed training for ethical conduct of research. All IRB approval was obtained before starting data collection. The respondents' identity was not revealed; moreover, there is no written mention in any public document of the name and or other indicators that identify the respondents. Only general demographic information was collected. Furthermore, no harm was done to the respondent physically, emotionally or in any other way, shape or form.

\section{Summary}

In Chapter III, the population of the study and the details of the sample were discussed. The procedure used for data collection and the different instruments utilized for data collection was also explained. Further, a detailed description of the analyses conducted by the researcher to test the hypotheses of the study was provided. In Chapter IV, that follows, the results of the analyses conducted by the researcher will be presented. 


\section{CHAPTER IV}

\section{RESULTS AND FINDINGS}

In this chapter, results from descriptive statistics, reliability analyses, factor analyses, regression analyses, path analysis are reported. SPSS 16.00 and AMOS were used to analyze the data.

Table 4 Question Legend

\begin{tabular}{|c|c|c|c|c|}
\hline Label & Section & Question & Description & Foundation \\
\hline CS1 & One & 1 & Career Support & $\begin{array}{c}\text { Perceived Organizational } \\
\text { Support }\end{array}$ \\
\hline $\mathrm{CS} 2$ & One & 2 & Career Support & $\begin{array}{c}\text { Perceived Organizational } \\
\text { Support }\end{array}$ \\
\hline $\mathrm{CS} 3$ & One & 3 & Career Support & $\begin{array}{c}\text { Perceived Organizational } \\
\text { Support }\end{array}$ \\
\hline PS1 & One & 4 & Psychosocial Support & $\begin{array}{c}\text { Perceived Organizational } \\
\text { Support }\end{array}$ \\
\hline PS2 & One & 5 & Psychosocial Support & $\begin{array}{c}\text { Perceived Organizational } \\
\text { Support }\end{array}$ \\
\hline PS3 & One & 6 & Psychosocial Support & $\begin{array}{c}\text { Perceived Organizational } \\
\text { Support }\end{array}$ \\
\hline IM1 & One & M1 & Intent to Mentor & Mentoring \\
\hline IM2 & One & M2 & Intent to Mentor & Mentoring \\
\hline JS1 & Two & 7 & Job Satisfaction & Affective Reaction \\
\hline JS2 & Two & 8 & Job Satisfaction & Affective Reaction \\
\hline JS3 & Two & 9 & Job Satisfaction & Affective Reaction \\
\hline OC1 & Three & 10 & $\begin{array}{c}\text { Organizational } \\
\text { Commitment }\end{array}$ & Affective Reaction \\
\hline $\mathrm{OC} 2$ & Three & 11 & $\begin{array}{c}\text { Organizational } \\
\text { Commitment }\end{array}$ & Affective Reaction \\
\hline OC3 & Three & 12 & $\begin{array}{l}\text { Organizational } \\
\text { Commitment }\end{array}$ & Affective Reaction \\
\hline OC4 & Three & 13 & $\begin{array}{c}\text { Organizational } \\
\text { Commitment }\end{array}$ & Affective Reaction \\
\hline
\end{tabular}


Table 4 continued.

\begin{tabular}{|c|c|c|c|c|}
\hline Label & Section & Question & Description & Foundation \\
\hline OC5 & Three & 14 & $\begin{array}{c}\text { Organizational } \\
\text { Commitment }\end{array}$ & Affective Reaction \\
\hline OC6 & Three & 15 & $\begin{array}{c}\text { Organizational } \\
\text { Commitment }\end{array}$ & Affective Reaction \\
\hline CV01 & Four & 16 & Individualism & Cultural Values \\
\hline CV02 & Four & 17 & Individualism & Cultural Values \\
\hline CV03 & Four & 18 & Power Distance & Cultural Values \\
\hline CV04 & Four & 19 & Individualism & Cultural Values \\
\hline CV05 & Four & 20 & Masculinity & Cultural Values \\
\hline CV06 & Four & 21 & Power Distance & Cultural Values \\
\hline CV07 & Four & 22 & Masculinity & Cultural Values \\
\hline CV08 & Four & 23 & Individualism & Cultural Values \\
\hline CV09 & Four & 24 & $\begin{array}{l}\text { Long Term } \\
\text { Orientation }\end{array}$ & Cultural Values \\
\hline CV10 & Four & 25 & $\begin{array}{l}\text { Long Term } \\
\text { Orientation }\end{array}$ & Cultural Values \\
\hline CV11 & Four & 26 & $\begin{array}{l}\text { Long Term } \\
\text { Orientation }\end{array}$ & Cultural Values \\
\hline CV12 & Four & 27 & $\begin{array}{l}\text { Long Term } \\
\text { Orientation }\end{array}$ & Cultural Values \\
\hline CV13 & Four & 28 & $\begin{array}{l}\text { Uncertainty } \\
\text { Avoidance }\end{array}$ & Cultural Values \\
\hline CV14 & Four & 29 & Power Distance & Cultural Values \\
\hline CV15 & Four & 30 & Masculinity & Cultural Values \\
\hline CV16 & Four & 31 & $\begin{array}{l}\text { Uncertainty } \\
\text { Avoidance }\end{array}$ & Cultural Values \\
\hline CV17 & Four & 32 & Power Distance & Cultural Values \\
\hline CV18 & Four & 33 & $\begin{array}{l}\text { Uncertainty } \\
\text { Avoidance }\end{array}$ & Cultural Values \\
\hline CV19 & Four & 34 & $\begin{array}{l}\text { Uncertainty } \\
\text { Avoidance }\end{array}$ & Cultural Values \\
\hline CV20 & Four & 35 & Masculinity & Cultural Values \\
\hline
\end{tabular}




\section{Descriptive Statistics}

SPSS was used to compute descriptive statistics for all 35 items. The descriptive statistics are shown in Table 5.

Table 5 Descriptive Statistics

\begin{tabular}{|c|c|c|c|c|c|c|c|c|}
\hline & N & Min & Max & Mean & S.D. & Skewness & $\begin{array}{c}\text { Respondent } \\
\text { Ratio }\end{array}$ \\
\hline CS1 & $\begin{array}{c}\text { Perceived } \\
\text { Organizational } \\
\text { Support }\end{array}$ & 341 & 1 & 7 & 4.43 & 1.9 & -0.462 & 0.997 \\
\hline CS2 & $\begin{array}{c}\text { Perceived } \\
\text { Organizational } \\
\text { Support }\end{array}$ & 341 & 1 & 7 & 4.58 & 1.87 & -0.524 & 0.991 \\
\hline CS3 & $\begin{array}{c}\text { Perceived } \\
\text { Organizational } \\
\text { Support }\end{array}$ & 341 & 1 & 7 & 4.09 & 1.88 & -0.241 & 0.982 \\
\hline PS1 & $\begin{array}{c}\text { Perceived } \\
\text { Organizational } \\
\text { Support }\end{array}$ & 341 & 1 & 7 & 3.73 & 1.9 & -0.009 & 0.985 \\
\hline PS2 & $\begin{array}{c}\text { Perceived } \\
\text { Organizational } \\
\text { Support }\end{array}$ & 341 & 1 & 7 & 3.96 & 1.88 & -0.217 & 0.991 \\
\hline PS3 & $\begin{array}{c}\text { Perceived } \\
\text { Organizational } \\
\text { Support }\end{array}$ & 341 & 1 & 7 & 4.51 & 1.85 & -0.48 & 0.991 \\
\hline IM1 & $\begin{array}{c}\text { Mentoring } \\
\text { JM2 }\end{array}$ & 341 & 1 & 2 & 1.36 & 0.45 & 0.603 & 0.997 \\
\hline $\begin{array}{c}\text { Affective Reaction } \\
\text { Affective Reaction }\end{array}$ & 341 & 1 & 7 & 5.18 & 1.68 & -0.995 & 0.997 \\
\hline $\begin{array}{c}\text { Affective Reaction } \\
\text { Mentoring }\end{array}$ & 341 & 1 & 7 & 5.69 & 1.49 & -1.471 & 12 \\
\hline
\end{tabular}


Table 5 continued.

\begin{tabular}{|c|c|c|c|c|c|c|c|c|}
\hline & & $\mathrm{N}$ & Min & Max & Mean & S.D. & Skewness & $\begin{array}{c}\text { Respondent } \\
\text { Ratio }\end{array}$ \\
\hline $\mathrm{OC} 1$ & Affective Reaction & 341 & 1 & 4 & 3.57 & 0.7 & -1.85 & 0.994 \\
\hline $\mathrm{OC} 2$ & Affective Reaction & 341 & 1 & 4 & 3.2 & 0.97 & -0.974 & 0.988 \\
\hline OC3 & Affective Reaction & 341 & 1 & 4 & 2.52 & 1 & -0.088 & 0.991 \\
\hline OC4 & Affective Reaction & 341 & 1 & 4 & 3.12 & 0.81 & -0.818 & 0.985 \\
\hline OC5 & Affective Reaction & 341 & 1 & 4 & 3.5 & 0.73 & -1.513 & 0.994 \\
\hline OC6 & Affective Reaction & 341 & 1 & 4 & 2.57 & 0.95 & -0.144 & 0.988 \\
\hline CV01 & Cultural Values & 341 & 1 & 5 & 2.56 & 1.37 & 0.528 & 0.997 \\
\hline CV02 & Cultural Values & 341 & 1 & 5 & 2.65 & 1.45 & 0.347 & 0.997 \\
\hline CV03 & Cultural Values & 341 & 1 & 5 & 2.58 & 1.55 & 0.467 & 0.997 \\
\hline CV04 & Cultural Values & 341 & 1 & 5 & 2.48 & 1.56 & 0.59 & 0.991 \\
\hline CV05 & Cultural Values & 341 & 1 & 5 & 2.55 & 1.54 & 0.558 & 0.994 \\
\hline CV06 & Cultural Values & 341 & 1 & 5 & 2.65 & 1.23 & 0.423 & 0.991 \\
\hline CV07 & Cultural Values & 341 & 1 & 5 & 2.45 & 1.7 & 0.606 & 0.997 \\
\hline
\end{tabular}


Table 5 continued.

\begin{tabular}{|c|c|c|c|c|c|c|c|c|}
\hline CV08 & Cultural Values & 341 & 1 & 5 & 2.63 & 1.5 & 0.424 & 0.997 \\
\hline CV09 & Cultural Values & 341 & 1 & 5 & 2.52 & 1.43 & 0.579 & 0.988 \\
\hline CV10 & Cultural Values & 341 & 1 & 5 & 2.67 & 1.2 & 0.422 & 0.988 \\
\hline CV11 & Cultural Values & 341 & 1 & 5 & 2.52 & 1.4 & 0.546 & 0.988 \\
\hline CV12 & Cultural Values & 341 & 1 & 5 & 2.69 & 1.26 & 0.349 & 0.991 \\
\hline CV13 & Cultural Values & 341 & 1 & 5 & 2.67 & 0.78 & 0.049 & 0.988 \\
\hline CV14 & Cultural Values & 341 & 1 & 5 & 3.07 & 1.08 & -0.078 & 0.988 \\
\hline CV15 & Cultural Values & 341 & 1 & 5 & 2.93 & 1.04 & -0.044 & 0.968 \\
\hline CV16 & Cultural Values & 341 & 1 & 5 & 3.28 & 1.25 & -0.249 & 0.971 \\
\hline CV17 & Cultural Values & 341 & 1 & 5 & 2.64 & 1.09 & 0.217 & 0.971 \\
\hline CV18 & Cultural Values & 341 & 1 & 5 & 2.99 & 1.16 & -0.098 & 0.968 \\
\hline CV19 & Cultural Values & 341 & 1 & 5 & 2.39 & 1.27 & 0.593 & 0.971 \\
\hline CV20 & Cultural Values & 341 & 1 & 5 & 2.89 & 1.19 & 0.145 & 0.974 \\
\hline & Time employed & 341 & & & & & & 0.971 \\
\hline
\end{tabular}


As the results in Table 5 indicate, the sample in this study included 341 respondents. The means and standard deviations for each item are shown in the above table. The means for career support, psychosocial support, job satisfaction, organizational commitment and cultural values were $4.366,4.063,5.508,3.079$, and 2.69 respectively.

\section{Results of Factor Analysis}

According to Tabachnick and Fidell (2001), the correlation (r), as found in the correlation matrix, should be .3 or higher in order to be considered in the same factor. Moreover, according to Tabachnick and Fidell (2001), Kaiser-Meyer-Olkin Measure of Sampling Adequacy (KMO) should be 0.6 or above and the Bartlett's Test of Sphericity should be significant (in this case 0.1 or smaller). All of the tests listed below in Table 6 satisfy these conditions. Therefore correlations of 0.3 or higher can be grouped together.

Table 6 Summary of Sampling Adequacy for Factors

\begin{tabular}{|l|c|c|}
\hline & KMO & Bartlett's Test Significance \\
\hline Career Support & 0.714 & 0.00 \\
\hline Psychosocial Support & 0.609 & 0.00 \\
\hline $\begin{array}{l}\text { Perceived Organizational } \\
\text { Support }\end{array}$ & 0.758 & 0.00 \\
\hline Job Satisfaction & 0.725 & 0.00 \\
\hline Organizational Commitment & 0.777 & 0.00 \\
\hline Affective Reaction & 0.872 & 0.00 \\
\hline Cultural Values & 0.951 & 0.00 \\
\hline
\end{tabular}


In the following Tables 7-20, the Principal Component Analysis extraction method was used.

Table 7 Total Variance Explained for Career Support

\begin{tabular}{|c|c|c|c|c|}
\hline & \multirow{2}{*}{ Component } & \multicolumn{3}{|c|}{ Initial Eigenvalues } \\
\hline & & Total & $\begin{array}{c}\text { \% of } \\
\text { Variance }\end{array}$ & $\begin{array}{c}\text { Cumulative } \\
\%\end{array}$ \\
\hline CS1 & $\begin{array}{l}\text {... someone at work who takes a personal interest } \\
\text { in my career. }\end{array}$ & 2.399 & 79.951 & 79.951 \\
\hline CS2 & ...helps me coordinate professional goals. & 0.402 & 13.393 & 93.344 \\
\hline CS3 & $\begin{array}{l}\text {...devotes special time and consideration to my } \\
\text { career. }\end{array}$ & 0.2 & 6.656 & 100 \\
\hline
\end{tabular}

Table 8 Component Matrix for Career Support

\begin{tabular}{|l|l|c|}
\hline & \multicolumn{1}{|c|}{ Variable } & Component \\
\hline CS1 & ...someone at work who takes a personal interest in my career. & 0.848 \\
\hline CS2 & $\ldots$ helps me coordinate professional goals. & 0.919 \\
\hline CS3 & $\ldots$. devotes special time and consideration to my career. & 0.914 \\
\hline
\end{tabular}

Table 9 Total Variance Explained for Psychosocial Support

\begin{tabular}{|c|c|c|c|c|}
\hline & \multirow{2}{*}{ Component } & \multicolumn{3}{|c|}{ Initial Eigenvalues } \\
\hline & & Total & $\begin{array}{c}\text { \% of } \\
\text { Variance }\end{array}$ & $\begin{array}{c}\text { Cumulative } \\
\%\end{array}$ \\
\hline PS1 & $\begin{array}{l}\text {...I share my personal problems with someone at } \\
\text { work. }\end{array}$ & 1.987 & 66.242 & 66.242 \\
\hline PS2 & $\begin{array}{l}\text {..exchange confidences with someone in a } \\
\text { senior position at work. }\end{array}$ & 0.686 & 22.865 & 89.107 \\
\hline PS3 & ...someone in a senior position to be a friend. & 0.327 & 10.893 & 100 \\
\hline
\end{tabular}


Table 10 Component Matrix for Psychosocial Support

\begin{tabular}{|l|l|c|}
\hline & \multicolumn{1}{|c|}{ Variable } & Component \\
\hline PS1 & $\ldots$ I share my personal problems with someone at work. & 0.719 \\
\hline PS2 & $\begin{array}{l}\text { f.exchange confidences with someone in a senior position at } \\
\text { work. }\end{array}$ & 0.892 \\
\hline PS3 & $\ldots$ someone in a senior position to be a friend. & 0.821 \\
\hline
\end{tabular}

Table 11 Total Variance Explained for Perceived Organizational Support

\begin{tabular}{|c|c|c|c|c|}
\hline & \multirow{2}{*}{ Component } & \multicolumn{3}{|c|}{ Initial Eigenvalues } \\
\hline & & Total & $\begin{array}{c}\text { \% of } \\
\text { Variance }\end{array}$ & $\underset{\%}{\text { Cumulative }}$ \\
\hline CS1 & $\begin{array}{l}\text {... someone at work who takes a personal interest } \\
\text { in my career. }\end{array}$ & 3.176 & 52.94 & 52.94 \\
\hline CS2 & ...helps me coordinate professional goals. & 1.226 & 20.432 & 73.372 \\
\hline CS3 & $\begin{array}{l}\text {...devotes special time and consideration to my } \\
\text { career. }\end{array}$ & 0.673 & 11.221 & 84.592 \\
\hline PS1 & $\begin{array}{l}\text {...I share my personal problems with someone at } \\
\text { work. }\end{array}$ & 0.41 & 6.84 & 91.432 \\
\hline PS2 & $\begin{array}{l}\text {...exchange confidences with someone in a } \\
\text { senior position at work. }\end{array}$ & 0.317 & 5.291 & 96.723 \\
\hline PS3 & ...someone in a senior position to be a friend. & 0.197 & 3.277 & 100 \\
\hline
\end{tabular}

Table 12 Component Matrix for Perceived Organizational Support

\begin{tabular}{|l|l|c|}
\hline & \multicolumn{1}{|c|}{ Variable } & Component \\
\hline CS1 & $\ldots$.. someone at work who takes a personal interest in my career. & 0.761 \\
\hline CS2 & $\ldots$.helps me coordinate professional goals. & 0.824 \\
\hline CS3 & $\ldots$. devotes special time and consideration to my career. & 0.816 \\
\hline PS1 & $\ldots$.. share my personal problems with someone at work. & 0.516 \\
\hline
\end{tabular}


Table 12 Continued.

\begin{tabular}{|c|c|c|}
\hline & Variable & Component \\
\hline PS2 & $\begin{array}{l}\text {...exchange confidences with someone in a senior position at } \\
\text { work. }\end{array}$ & 0.706 \\
\hline PS3 & ...someone in a senior position to be a friend. & 0.699 \\
\hline
\end{tabular}

Table 13 Total Variance Explained for Job Satisfaction

\begin{tabular}{|c|c|c|c|c|}
\hline & \multirow{2}{*}{ Component } & \multicolumn{3}{|c|}{ Initial Eigenvalues } \\
\hline & & Total & $\begin{array}{c}\text { \% of } \\
\text { Variance }\end{array}$ & $\begin{array}{c}\text { Cumulative } \\
\%\end{array}$ \\
\hline JS1 & ...satisfied with my job. & 2.253 & 75.084 & 75.084 \\
\hline JS2 & ...I like my job. & 0.389 & 12.958 & 88.042 \\
\hline JS3 & ...I like working here. & 0.359 & 11.958 & 100 \\
\hline
\end{tabular}

Table 14 Component Matrix for Job Satisfaction

\begin{tabular}{|l|l|c|}
\hline & \multicolumn{1}{|c|}{ Variable } & Component \\
\hline JS1 & ...satisfied with my job. & 0.871 \\
\hline JS2 & $\ldots$ I like my job. & 0.869 \\
\hline JS3 & $\ldots$ I like working here. & 0.86 \\
\hline
\end{tabular}

Table 15 Total Variance Explained for Organizational Commitment

\begin{tabular}{|c|c|c|c|c|}
\hline & \multirow{2}{*}{ Component } & \multicolumn{3}{|c|}{ Initial Eigenvalues } \\
\hline & & Total & $\begin{array}{c}\text { \% of } \\
\text { Variance }\end{array}$ & $\underset{\%}{\text { Cumulative }}$ \\
\hline OC1 & $\begin{array}{l}\ldots \text { work harder than I have to in order to help } \\
\text { this organization succeed. }\end{array}$ & 2.542 & 42.363 & 42.363 \\
\hline OC2 & ... I feel loyal to this organization. & 1.012 & 16.873 & 59.235 \\
\hline OC3 & $\begin{array}{l}\text {...take almost any job to keep working for this } \\
\text { organization. }\end{array}$ & 0.804 & 13.406 & 72.641 \\
\hline
\end{tabular}


Table 15 Continued.

\begin{tabular}{|c|c|c|c|c|}
\hline & \multirow{2}{*}{ Component } & \multicolumn{3}{|c|}{ Initial Eigenvalues } \\
\hline & & Total & $\begin{array}{c}\text { \% of } \\
\text { Variance }\end{array}$ & $\begin{array}{c}\text { Cumulative } \\
\%\end{array}$ \\
\hline OC4 & $\begin{array}{l}\ldots \text { my values and the agency's values are very } \\
\text { similar. }\end{array}$ & 0.664 & 11.066 & 83.707 \\
\hline OC5 & ... proud to be working for this organization. & 0.539 & 8.982 & 92.689 \\
\hline OC6 & $\begin{array}{l}\text {.. turn down another job for more pay in order } \\
\text { to stay with this organization. }\end{array}$ & 0.439 & 7.311 & 100 \\
\hline
\end{tabular}

Table 16 Component Matrix for Organizational Commitment

\begin{tabular}{|l|l|c|}
\hline & \multicolumn{1}{|c|}{ Variable } & Component \\
\hline OC1 & $\begin{array}{l}\text { f. work harder than I have to in order to help this organization } \\
\text { succeed. }\end{array}$ & 0.678 \\
\hline OC2 & $\ldots$ I feel loyal to this organization. & 0.452 \\
\hline OC3 & $\ldots$ take almost any job to keep working for this organization. & 0.506 \\
\hline OC4 & $\ldots$ my values and the agency's values are very similar. & 0.77 \\
\hline OC5 & $\ldots$ proud to be working for this organization. & 0.804 \\
\hline OC6 & $\begin{array}{l}\text {.. turn down another job for more pay in order to stay with this } \\
\text { organization. }\end{array}$ & 0.617 \\
\hline
\end{tabular}

Table 17 Total Variance Explained for Affective Reaction

\begin{tabular}{|c|c|c|c|c|}
\hline & \multirow{2}{*}{ Component } & \multicolumn{3}{|c|}{ Initial Eigenvalues } \\
\hline & & Total & $\begin{array}{c}\text { \% of } \\
\text { Variance } \\
\end{array}$ & $\begin{array}{c}\text { Cumulative } \\
\%\end{array}$ \\
\hline JS1 & ...satisfied with my job. & 3.921 & 43.572 & 43.572 \\
\hline JS2 & ...I like my job. & 1.036 & 11.509 & 55.08 \\
\hline JS3 & ...I like working here. & 0.929 & 10.326 & 65.406 \\
\hline
\end{tabular}


Table 17 Continued.

\begin{tabular}{|c|c|c|c|c|}
\hline & \multirow{2}{*}{ Component } & \multicolumn{3}{|c|}{ Initial Eigenvalues } \\
\hline & & Total & $\begin{array}{c}\text { \% of } \\
\text { Variance }\end{array}$ & $\begin{array}{c}\text { Cumulative } \\
\%\end{array}$ \\
\hline OC1 & $\begin{array}{l}\ldots \text { work harder than I have to in order to help } \\
\text { this organization succeed. }\end{array}$ & 0.764 & 8.492 & 73.898 \\
\hline OC2 & ... I feel loyal to this organization. & 0.663 & 7.372 & 81.27 \\
\hline OC3 & $\begin{array}{l}\text {...take almost any job to keep working for } \\
\text { this organization. }\end{array}$ & 0.545 & 6.052 & 87.322 \\
\hline OC4 & $\begin{array}{l}\ldots \text { my values and the agency's values are very } \\
\text { similar. }\end{array}$ & 0.444 & 4.929 & 92.251 \\
\hline OC5 & ... proud to be working for this organization. & 0.357 & 3.964 & 96.215 \\
\hline OC6 & $\begin{array}{l}\ldots \text { turn down another job for more pay in } \\
\text { order to stay with this organization. }\end{array}$ & 0.341 & 3.785 & 100 \\
\hline
\end{tabular}

Table 18 Component Matrix for Perceived Organizational Support

\begin{tabular}{|c|c|c|}
\hline & Variable & Component \\
\hline JS1 & ...satisfied with my job. & 0.747 \\
\hline JS2 & ...I like my job. & 0.754 \\
\hline JS3 & ...I like working here. & 0.806 \\
\hline OC1 & $\begin{array}{l}\text {... work harder than I have to in order to help this organization } \\
\text { succeed. }\end{array}$ & 0.662 \\
\hline OC2 & ... I feel loyal to this organization. & 0.421 \\
\hline OC3 & ...take almost any job to keep working for this organization. & 0.473 \\
\hline OC4 & ... my values and the agency's values are very similar. & 0.682 \\
\hline OC5 & ... proud to be working for this organization. & 0.756 \\
\hline OC6 & $\begin{array}{l}\text {.. turn down another job for more pay in order to stay with this } \\
\text { organization. }\end{array}$ & 0.519 \\
\hline
\end{tabular}


Table 19 Total Variance Explained for Cultural Values

\begin{tabular}{|c|c|c|c|c|}
\hline & \multirow{2}{*}{ Component } & \multicolumn{3}{|c|}{ Initial Eigenvalues } \\
\hline & & Total & $\begin{array}{c}\% \text { of } \\
\text { Variance }\end{array}$ & $\underset{\%}{\text { Cumulative }}$ \\
\hline CV01 & ... sufficient time for my personal or family life. & 8.933 & 44.666 & 44.666 \\
\hline CV02 & $\begin{array}{l}\text {... good physical working conditions (good } \\
\text { ventilation and lighting, adequate work space, } \\
\text { etc.). }\end{array}$ & 1.4 & 6.998 & 51.664 \\
\hline CV03 & $\begin{array}{l}\ldots \text { good working relationship with my direct } \\
\text { supervisor. }\end{array}$ & 1.304 & 6.518 & 58.182 \\
\hline CV04 & ...security of employment. & 1.16 & 5.801 & 63.983 \\
\hline CV05 & ... people who cooperate well with one another. & 1.109 & 5.545 & 69.528 \\
\hline CV06 & $\begin{array}{l}\text {.. consulted by my direct superior in his/her } \\
\text { decisions. }\end{array}$ & 0.962 & 4.811 & 74.339 \\
\hline CV07 & $\begin{array}{l}\text {.. opportunity for advancement to higher level } \\
\text { jobs. }\end{array}$ & 0.831 & 4.153 & 78.492 \\
\hline CV08 & ... element of variety and adventure in the job. & 0.765 & 3.826 & 82.318 \\
\hline CV09 & Personal steadiness and stability & 0.697 & 3.483 & 85.801 \\
\hline CV10 & Thrift (frugal, economically conscious lifestyle) & 0.667 & 3.337 & 89.137 \\
\hline CV11 & Persistence (perseverance) & 0.4 & 2.001 & 91.138 \\
\hline CV12 & Respect for Tradition & 0.34 & 1.701 & 92.839 \\
\hline CV13 & How often do you feel nervous or tense at work? & 0.277 & 1.384 & 94.223 \\
\hline
\end{tabular}


Table 19 continued.

\begin{tabular}{|c|c|c|c|c|}
\hline & \multirow{2}{*}{ Component } & \multicolumn{3}{|c|}{ Initial Eigenvalues } \\
\hline & & Total & $\begin{array}{c}\text { \% of } \\
\text { Variance }\end{array}$ & $\underset{\%}{\text { Cumulative }}$ \\
\hline CV14 & $\begin{array}{l}\text {... subordinates afraid to express disagreement } \\
\text { with their super... }\end{array}$ & 0.266 & 1.329 & 95.552 \\
\hline CV15 & Most people can be trusted. & 0.199 & 0.997 & 96.549 \\
\hline CV16 & $\begin{array}{l}\text {... without having precise answers to most } \\
\text { questions that subordinates may raise about their } \\
\text { work. }\end{array}$ & 0.174 & 0.87 & 97.419 \\
\hline CV17 & ... two bosses should be avoided at all costs. & 0.169 & 0.844 & 98.263 \\
\hline CV18 & $\begin{array}{l}\text { Competition between employees usually does } \\
\text { more harm than good. }\end{array}$ & 0.146 & 0.73 & 98.993 \\
\hline CV19 & $\begin{array}{l}\text {... rules should not be broken- not even when the } \\
\text { employee thinks it is in the company's best } \\
\text { interest. }\end{array}$ & 0.109 & 0.543 & 99.536 \\
\hline CV20 & $\begin{array}{l}\text { When people have failed in life it is often their } \\
\text { own fault. }\end{array}$ & 0.093 & 0.464 & 100 \\
\hline
\end{tabular}

Table 20 Component Matrix for Cultural Values

\begin{tabular}{|c|c|c|c|c|}
\hline & Variable & $\begin{array}{c}\text { Component } \\
1\end{array}$ & $\begin{array}{c}\text { Component } \\
2\end{array}$ & $\begin{array}{c}\text { Component } \\
3\end{array}$ \\
\hline CV01 & $\begin{array}{l}\text {.. sufficient time for my personal or } \\
\text { family life. }\end{array}$ & 0.804 & & \\
\hline CV02 & $\begin{array}{l}\text {... good physical working conditions (good } \\
\text { ventilation and lighting, adequate work } \\
\text { space, etc.). }\end{array}$ & 0.861 & & \\
\hline CV03 & $\begin{array}{l}\text {... good working relationship with my } \\
\text { direct supervisor. }\end{array}$ & 0.922 & & \\
\hline
\end{tabular}


Table 20 Continued.

\begin{tabular}{|c|c|c|c|c|}
\hline & Variable & $\begin{array}{c}\text { Component } \\
1\end{array}$ & $\begin{array}{c}\text { Component } \\
2\end{array}$ & $\begin{array}{l}\text { Component } \\
3\end{array}$ \\
\hline CV04 & ...security of employment. & 0.911 & & \\
\hline CV05 & $\begin{array}{l}\text {.. people who cooperate well with one } \\
\text { another. }\end{array}$ & 0.916 & & \\
\hline CV06 & $\begin{array}{l}\text {... consulted by my direct superior in } \\
\text { his/her decisions. }\end{array}$ & 0.815 & & \\
\hline CV07 & $\begin{array}{l}\text {... opportunity for advancement to higher } \\
\text { level jobs. }\end{array}$ & 0.913 & & \\
\hline CV08 & $\begin{array}{l}\text {... element of variety and adventure in the } \\
\text { job. }\end{array}$ & 0.876 & & \\
\hline CV09 & Personal steadiness and stability & 0.852 & & \\
\hline CV10 & $\begin{array}{l}\text { Thrift (frugal, economically conscious } \\
\text { lifestyle) }\end{array}$ & 0.746 & & \\
\hline CV11 & Persistence (perseverance) & 0.844 & & \\
\hline CV12 & Respect for Tradition & 0.71 & & \\
\hline CV13 & $\begin{array}{l}\text { How often do you feel nervous or tense at } \\
\text { work? }\end{array}$ & & & 0.522 \\
\hline CV14 & $\begin{array}{l}\ldots \text { subordinates afraid to express } \\
\text { disagreement with their super... }\end{array}$ & & & 0.343 \\
\hline CV15 & Most people can be trusted. & & & 0.738 \\
\hline CV16 & $\begin{array}{l}\ldots \text { without having precise answers to most } \\
\text { questions that subordinates may raise about } \\
\text { their work. }\end{array}$ & & & 0.38 \\
\hline
\end{tabular}


Table 20 Continued.

\begin{tabular}{|c|c|c|c|c|}
\hline & Variable & $\begin{array}{c}\text { Component } \\
1\end{array}$ & $\begin{array}{c}\text { Component } \\
2\end{array}$ & $\begin{array}{c}\text { Component } \\
3\end{array}$ \\
\hline CV17 & $\begin{array}{l}\text {... two bosses should be avoided at all } \\
\text { costs. }\end{array}$ & 0.302 & 0.477 & \\
\hline CV18 & $\begin{array}{l}\text { Competition between employees usually } \\
\text { does more harm than good. }\end{array}$ & & 0.331 & \\
\hline CV19 & $\begin{array}{l}\text {... rules should not be broken- not even } \\
\text { when the employee thinks it is in the } \\
\text { company's best interest. }\end{array}$ & 0.34 & 0.324 & \\
\hline CV20 & $\begin{array}{l}\text { When people have failed in life it is often } \\
\text { their own fault. }\end{array}$ & 0.078 & 0.099 & 0.202 \\
\hline
\end{tabular}

The factors for cultural values fall into three groups, the first group consists of the majority of the cultural values as would be expected. The other two groups consist primarily of the elements MAS and UAI indices. These dimensions were problematic in terms of both reliability and skewness (See Table 5).

\section{Estimates of Reliability}

The reliability estimates for mentoring, job satisfaction, organizational commitment, perceived organizational support, affective reaction and cultural values were computed using the Cronbach's alpha technique. The results of the analysis are provided in Table 21.

Table 21 Reliability Estimates

\begin{tabular}{|l|c|c|}
\hline \multicolumn{1}{|c|}{ Variable } & $\begin{array}{c}\text { Cronbach's } \\
\text { Alpha }\end{array}$ & Note \\
\hline All study items & 0.865 & \\
\hline
\end{tabular}


Table 21 Continued.

\begin{tabular}{|l|c|l|}
\hline \multicolumn{1}{|c|}{ Variable } & $\begin{array}{c}\text { Cronbach's } \\
\text { Alpha }\end{array}$ & Note \\
\hline Career Support & 0.874 & \\
\hline Psychosocial Support & 0.740 & \\
\hline Perceived Organizational Support & 0.812 & \\
\hline Job Satisfaction (6.1-6.3) & 0.829 & \\
\hline Organizational Commitment (7.1-7.6) & 0.693 & \\
\hline Affective Reaction & 0.812 & \\
\hline Cultural Values & 0.905 & \\
\hline IDV elements & 0.924 & \\
\hline PDI elements & 0.515 & \\
\hline MAS elements & 0.567 & \\
\hline LTO elements & 0.917 & \\
\hline UAI elements & -0.098 & Neg covariance between terms \\
\hline
\end{tabular}

The reliability (Cronbach's Alpha) of the PDI, MAS and particularly the UAI elements do not meet the expected minimum of 0.8 . This is due to extreme cultural shifts, especially in the case of the UAI elements, among the various countries and regions. These shifts are evident in the skewness in the Descriptive Statistics in Table 5.

A reliability estimate for the total instrument and each of the item sets was conducted using the data. Cronbach's alpha reliability estimate for the whole instrument was 0.865 , career support 0.874 , psychosocial support 0.740 , perceived organizational support 0.812 (MFQ), job satisfaction (OJSS) 0.829; organizational commitment (OCQ) 0.693, affective reaction (0.812), 
and finally for cultural values 0.905 . A reliability estimate was also run for each cultural index: Power Distance (PDI) 0.515, Individualism (IDV) 0.924, Masculinity (MAS) 0.567, Uncertainty Avoidance (UAI) -0.098 [a negative covariance exists between the terms), and Long-Term Orientation (LTO) 0.917.

\section{Results by Hypothesis}

\section{Research Hypothesis 1}

Employees' cultural values will significantly differ among employees in an oilfield services corporation - across Hofstede's (1980) five cultural dimensions. The researcher found neither the component variable nor Hofstede's indices to be normal, see normality tests in Appendix A-5. Moreover, a review of the literature did not provide a consensus as to whether the ANOVA is an appropriate test for Hofstede's indices; as such, both the ANOVA and Welch tests were used.

Table 22 Summary of p-value - ANOVA Significance - for Hofstede's indices

\begin{tabular}{|l|c|c|c|c|c|}
\hline & IDV & PDI & MAS & LTO & UAI \\
\hline Region & 0.461 & 0.453 & 0.009 & 0.428 & 0.236 \\
\hline Hemisphere & 0.249 & 0.357 & 0.998 & 0.102 & 0.024 \\
\hline
\end{tabular}

The only indices that have a significant difference (below 0.1) are Masculinity (MAS) by region and Uncertainty Avoidance (UAI) by hemisphere. Based on the ANOVA test, Hypothesis 1 is supported only in the case of MAS across regions and UAI across hemispheres. 
Table 23 Summary of p-value - Welch Significance - for Hofstede's indices

\begin{tabular}{|l|c|c|c|c|c|}
\hline & IDV & PDI & MAS & LTO & UAI \\
\hline Region & 0.478 & 0.476 & 0.006 & 0.329 & 0.249 \\
\hline Hemisphere & 0.259 & 0.362 & 0.998 & 0.095 & 0.023 \\
\hline
\end{tabular}

The Welch test showed significant difference for Long-Term Orientation (LTO) by hemisphere ( $\mathrm{p}$ value of $0.095<0.1$ ) and Uncertainty Avoidance (UAI) by hemisphere ( $\mathrm{p}$ value of $0.023<$ 0.1). Neither the ANOVA nor Welch test supports Hypothesis 1.

\section{Research Hypothesis 2}

Employees' individual cultural values - as framed by Hofstede (1980) - will be unrelated to their perceptions of support from their organization. Analysis by region and hemisphere will follow in the second portion of Hypothesis 2. Organizational Support and Interest in Mentoring is shown in Figure 2.

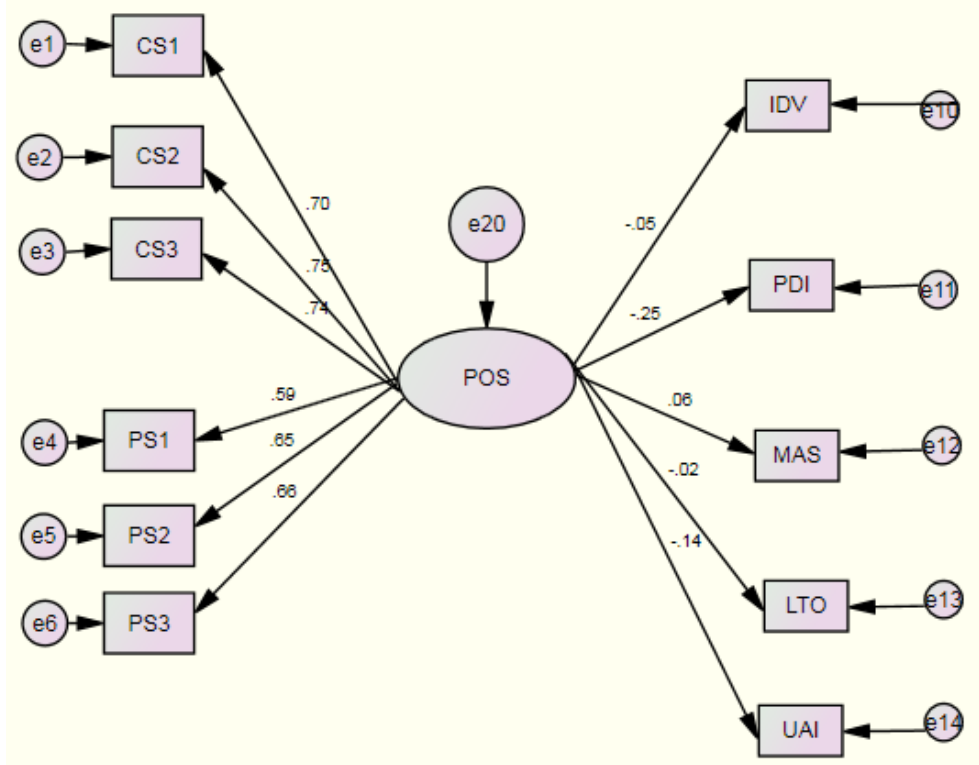

Figure 2 Structural Model of Organizational Support and Interest in Mentoring 
Table 24 Model Fit Indices of Structural Model of Organizational Support Cultural Values

\begin{tabular}{|l|c|c|c|}
\hline Model & GFI & NFI & CFI \\
\hline Default model & 0.8 & 0.621 & 0.65 \\
\hline Saturated model & 1 & 1 & 1 \\
\hline Independence model & 0.608 & 0 & 0 \\
\hline
\end{tabular}

AGFI of 0.8 is lower than that required by Tabachnick \& Fidell (2001) for the model to be a good fit. The NFI is 0.621 , lower than the 0.9 suggested by Bentler and Bonett (1980) for the model to be a good fit. A CFI of 0.65 is not close to 1 as suggested by Arbuckle (2010) for the model to be good fit. As none of these criteria have been met, the model tested is not a good fit.

Table 25 Summary Table for Perceived Organizational Support and Cultural Values

\begin{tabular}{|l|c|}
\hline & r \\
\hline Perceived Organizational Support and Individual Index (IDV) - 2a & 0.05 \\
\hline Perceived Organizational Support and Power Index (PDI) - 2b & 0.25 \\
\hline Perceived Organizational Support and Uncertainty Avoidance Index (UAI) - 2c & 0.14 \\
\hline Perceived Organizational Support and Masculinity Index (MAS) - 2d & 0.06 \\
\hline Perceived Organizational Support and Long-term Orientation Index (LTO) - 2e & 0.02 \\
\hline
\end{tabular}

The highest correlation was 0.25 , there is not a significant relationship between perceived organizational support and the individual cultural values; therefore, Hypothesis 2 is supported. Perceived Organizational Support and Cultural Values are shown in Figure 3 and Table 26. 


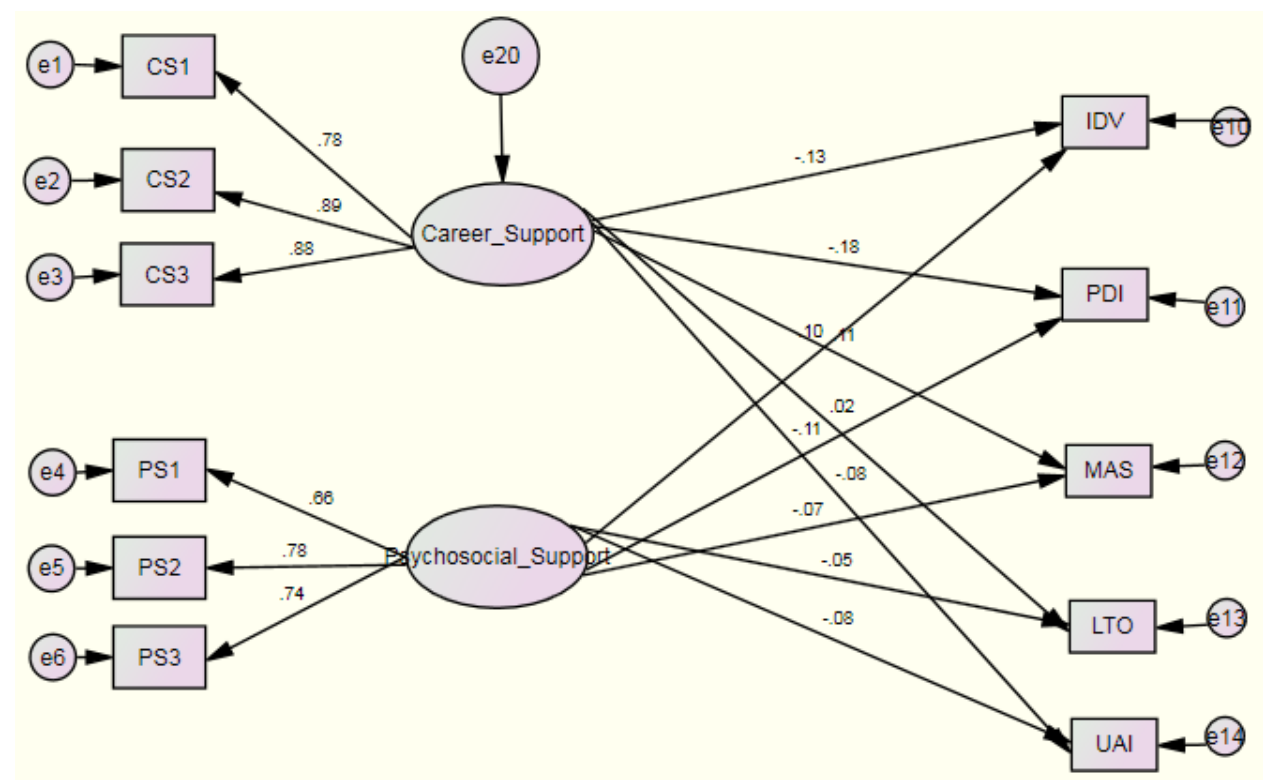

Figure 3 Structural Model of Perceived Organizational Support and Cultural Values

With a GFI of 0.914 , a NFI of 0.818 , and a CFI of 0.852 ; two of three criteria have been met; as such, the model tested should be a good fit.

Table 26 Model Fit Indices of Structural Model of Career and Psychosocial Support Cultural Values

\begin{tabular}{|l|c|c|c|}
\hline \multicolumn{1}{|c|}{ Model } & GFI & NFI & CFI \\
\hline Default model & 0.914 & 0.818 & 0.852 \\
\hline Saturated model & 1 & 1 & 1 \\
\hline Independence model & 0.608 & 0 & 0 \\
\hline
\end{tabular}

The Structural Model of Perceived Organizational Support, broken up by Career and Psychosocial Support, and Cultural Values are shown in Table 27 and Table 28. 
Table 27 Summary Table for Career Support and Cultural Values

\begin{tabular}{|l|c|}
\hline & $\mathrm{r}$ \\
\hline Career Support and Individual Index (IDV) - 2a & -0.13 \\
\hline Career Support and Power Index (PDI) - 2b & -0.18 \\
\hline Career Support and Uncertainty Avoidance Index (UAI) - 2c & -0.11 \\
\hline Career Support and Masculinity Index (MAS) - 2d & 0.1 \\
\hline Career Support and Long-term Orientation Index (LTO) - 2e & 0.02 \\
\hline
\end{tabular}

Table 28 Summary Table for Psychosocial Support and Cultural Values

\begin{tabular}{|l|c|}
\hline & $\mathrm{r}$ \\
\hline Psychosocial Support and Individual Index (IDV) - 2a & 0.11 \\
\hline Psychosocial Support and Power Index (PDI) - 2b & -0.11 \\
\hline Psychosocial Support and Uncertainty Avoidance Index (UAI) - 2c & 0.08 \\
\hline Psychosocial Support and Masculinity Index (MAS) - 2d & -0.07 \\
\hline Psychosocial Support and Long-term Orientation Index (LTO) - 2e & 0.05 \\
\hline
\end{tabular}

The highest correlation between career support and the individual cultural values was 0.18 ; as such, there is not a significant relationship between career support and the individual cultural values. The highest correlation between psychosocial support and the individual cultural values was 0.11 ; as such, there is not a significant relationship between psychosocial support and the individual values. This supports Hypothesis 2a-e.

Breakdown by Region and Hemisphere - Research Hypothesis 2

Employees' individual cultural values (studied by Region) - as framed by Hofstede (1980) - will be unrelated to their perceptions of support from their organization. 
Due to properties of the smaller data set, estimated means and intercepts were used which negates the GFI test statistic. Organizational Support and Cultural Values for Africa are shown in Figure 4 and Table 29 and Table 30.

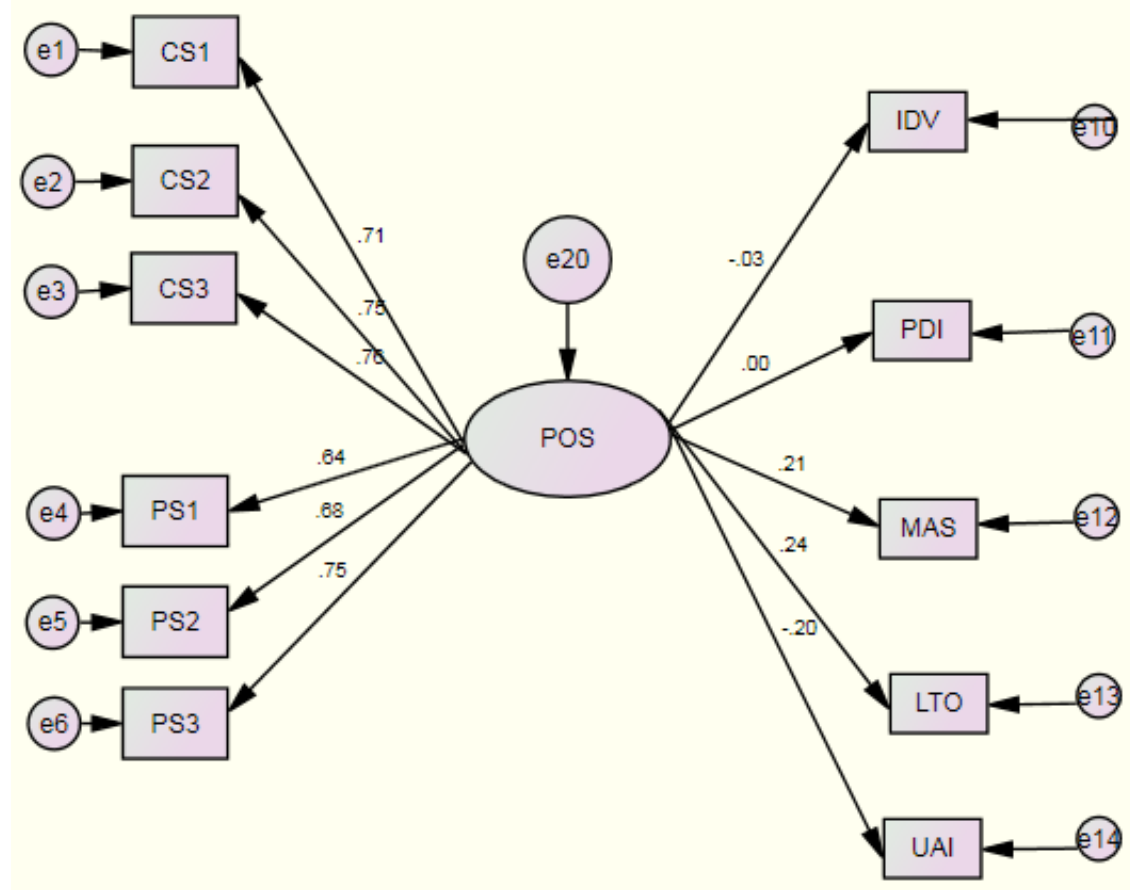

Figure 4 Structural Model of Perceived Organizational Support and Cultural Values for Africa

Table 29 Model Fit Indices of Structural Model of Perceived Organizational Support and Cultural Values for Africa

\begin{tabular}{|l|c|c|c|}
\hline \multicolumn{1}{|c|}{ Model } & GFI & NFI & CFI \\
\hline Default model & NA & 0.555 & 0.695 \\
\hline Saturated model & & 1 & 1 \\
\hline Independence model & & 0 & 0 \\
\hline
\end{tabular}

Neither the NFI nor the CFI are at a level to suggest that the model is a good fit. 
Table 30 Summary Table for Perceived Organizational Support and Cultural Values - Africa

\begin{tabular}{|l|c|}
\hline & $\mathrm{r}$ \\
\hline Perceived Organizational Support and Individual Index (IDV) - 2a & -0.03 \\
\hline Perceived Organizational Support and Power Index (PDI) - 2b & 0 \\
\hline Perceived Organizational Support and Uncertainty Avoidance Index (UAI) - 2c & -0.2 \\
\hline Perceived Organizational Support and Masculinity Index (MAS) - 2d & 0.21 \\
\hline Perceived Organizational Support and Long-term Orientation Index (LTO) - 2e & 0.24 \\
\hline
\end{tabular}

The highest correlation was 0.24 ; as such, there is not a significant relationship between perceived organizational support and the individual cultural values. Therefore, Hypothesis 2 is supported. While the model used is not a good fit, the results for the Africa region support Hypothesis 2.

Organizational Support and Cultural Values for Asia Pacific are shown in Figure 5 and Table 31 and Table 32.

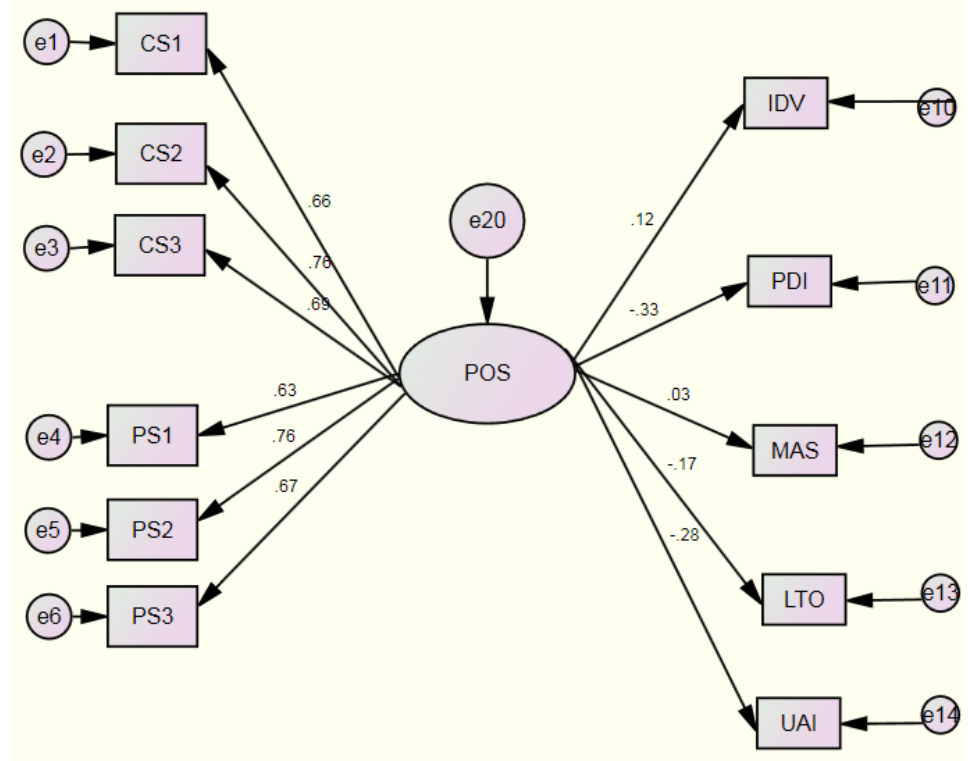

Figure 5 Structural Model of Perceived Organizational Support and Cultural Values Asia Pacific 
Table 31 Model Fit Indices of Structural Model of Perceived Organizational Support and Cultural Values -Asia Pacific

\begin{tabular}{|l|c|c|c|}
\hline \multicolumn{1}{|c|}{ Model } & GFI & NFI & CFI \\
\hline Default model & NA & 0.564 & 0.68 \\
\hline Saturated model & & 1 & 1 \\
\hline Independence model & & 0 & 0 \\
\hline
\end{tabular}

Neither the NFI nor the CFI are at a level to suggest that the model is a good fit.

Table 32 Summary Table for Perceived Organizational Support and Cultural Values - Asia Pacific

\begin{tabular}{|l|c|}
\hline & $\mathrm{r}$ \\
\hline Perceived Organizational Support and Individual Index (IDV) - 2a & 0.12 \\
\hline Perceived Organizational Support and Power Index (PDI) - 2b & -0.33 \\
\hline Perceived Organizational Support and Uncertainty Avoidance Index (UAI) - 2c & -0.28 \\
\hline Perceived Organizational Support and Masculinity Index (MAS) - 2d & 0.03 \\
\hline Perceived Organizational Support and Long-term Orientation Index (LTO) - 2e & -0.17 \\
\hline
\end{tabular}

There appears to be a significant negative relationship between perceived organizational support and the power distance (PDI) /uncertainty avoidance (UAI) indices. This does not support hypothesis $2 \mathrm{~b}$ and $2 \mathrm{c}$. As the other correlations are not significant, Hypothesis $2 \mathrm{a}, 2 \mathrm{~d}$, and $2 \mathrm{e}$ are supported. The model used is not a good fit; the results for the Asia Pacific region do not support Hypothesis 2.

Organizational Support and Cultural Values for Europe are shown in Figure 6 and Table 33 and Table 34. 


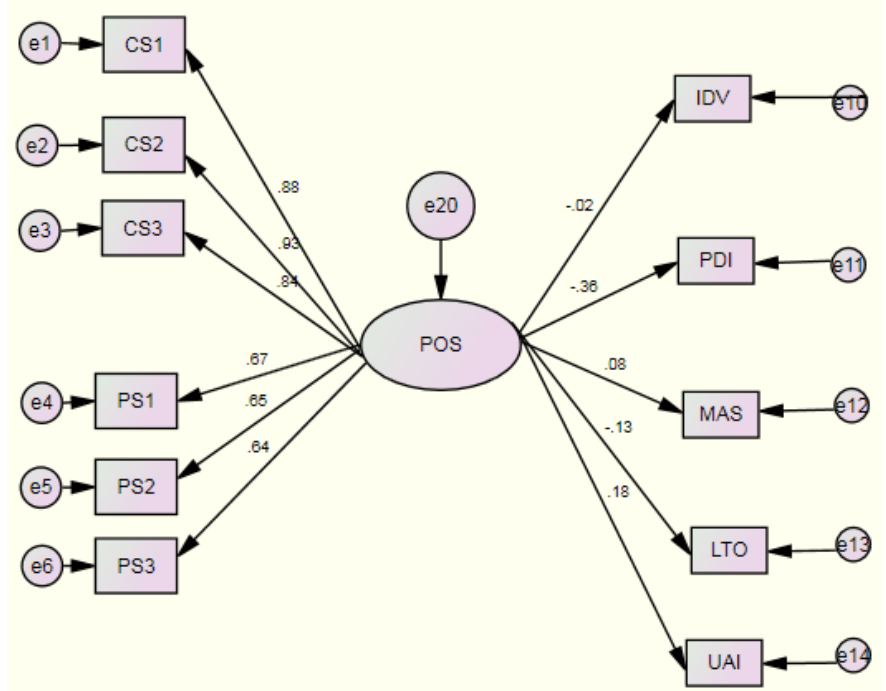

Figure 6 Structural Model of Perceived Organizational Support and Cultural Values - Europe

Table 33 Model Fit Indices of Structural Model of Perceived Organizational and Support Cultural Values - Europe

\begin{tabular}{|l|c|c|c|}
\hline \multicolumn{1}{|c|}{ Model } & GFI & NFI & CFI \\
\hline Default model & 0.656 & 0.448 & 0.622 \\
\hline Saturated model & 1 & 1 & 1 \\
\hline Independence model & 0.487 & 0 & 0 \\
\hline
\end{tabular}

None of the three criteria (GFI, NFI and CFI) are at a level to suggest that the model is a good fit. As none of these criteria have been met, the model tested is not a good fit.

Table 34 Summary Table for Organizational Support and Cultural Values - Europe

\begin{tabular}{|l|c|}
\hline & $\mathrm{r}$ \\
\hline Perceived Organizational Support and Individual Index (IDV) - 2a & -0.02 \\
\hline Perceived Organizational Support and Power Index (PDI) - 2b & -0.36 \\
\hline Perceived Organizational Support and Uncertainty Avoidance Index (UAI) - 2c & 0.18 \\
\hline Perceived Organizational Support and Masculinity Index (MAS) - 2d & 0.08 \\
\hline Perceived Organizational Support and Long-term Orientation Index (LTO) - 2e & -0.13 \\
\hline
\end{tabular}


There appears to be a significant negative relationship between perceived organizational support and the power distance (PDI) index. This does not support Hypothesis 2b. As the other correlations are not significant, Hypothesis $2 \mathrm{a}, 2 \mathrm{c}, 2 \mathrm{~d}$ and $2 \mathrm{e}$ are supported. The model used is not a good fit; the results for the Europe region do not support Hypothesis 2.

Organizational Support and Cultural Values for Latin America are shown in Figure 7 and Table 35 and Table 36.

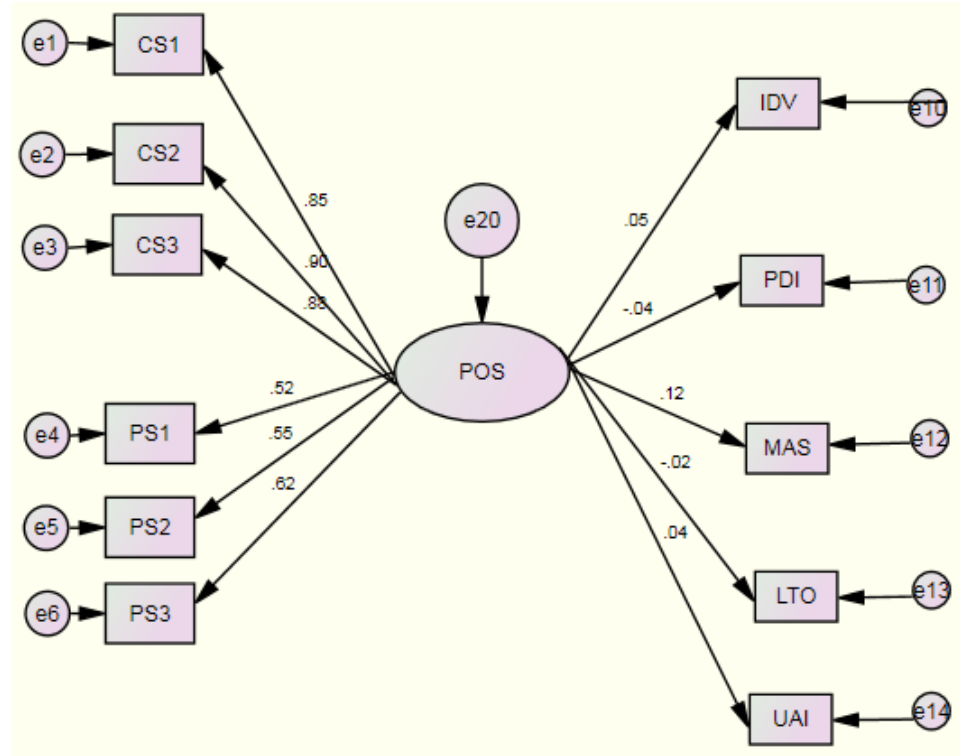

Figure 7 Structural Model of Perceived Organizational Support and Cultural Values - Latin America

Table 35 Model Fit Indices of Structural Model of Perceived Organizational Support and Cultural Values -Latin America

\begin{tabular}{|l|c|c|c|}
\hline \multicolumn{1}{|c|}{ Model } & GFI & NFI & CFI \\
\hline Default model & NA & 0.338 & 0.406 \\
\hline Saturated model & & 1 & 1 \\
\hline Independence model & & 0 & 0 \\
\hline
\end{tabular}


None of the criteria (NFI and CFI) are at a level to suggest that the model is a good fit.

Table 36 Summary Table for Perceived Organizational Support and Cultural Values - Latin America

\begin{tabular}{|l|c|}
\hline & r \\
\hline Perceived Organizational Support and Individual Index (IDV) - 2a & 0.05 \\
\hline Perceived Organizational Support and Power Index (PDI) - 2b & -0.04 \\
\hline Perceived Organizational Support and Uncertainty Avoidance Index (UAI) - 2c & 0.04 \\
\hline Perceived Organizational Support and Masculinity Index (MAS) - 2d & 0.12 \\
\hline Perceived Organizational Support and Long-term Orientation Index (LTO) - 2e & -0.02 \\
\hline
\end{tabular}

A significant relationship between perceived organizational support and the indices IDV, PDI, UAI, MAS, and LTO is not apparent. This supports Hypothesis $2 \mathrm{a}, 2 \mathrm{~b}, 2 \mathrm{c}, 2 \mathrm{~d}$ and $2 \mathrm{e}$. The highest correlation was 0.12 ; as such, there is not a significant relationship between perceived organizational support and the individual cultural values. Therefore, Hypothesis 2 is supported for Latin America.

Organizational Support and Cultural Values for Middle East are shown in Figure 8 and Table 37 and Table 38. 


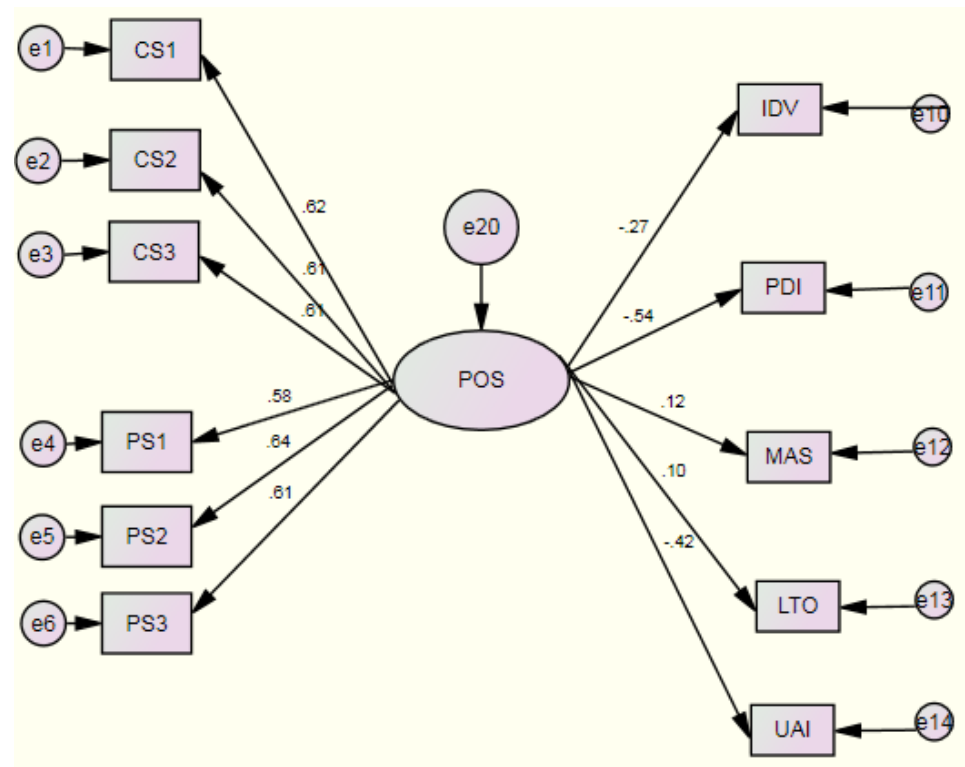

Figure 8 Structural Model of Perceived Organizational Support and Cultural Values - Middle East

Table 37 Model Fit Indices of Structural Model of Perceived Organizational Support and Cultural Values -Middle East

\begin{tabular}{|l|c|c|c|}
\hline \multicolumn{1}{|c|}{ Model } & GFI & NFI & CFI \\
\hline Default model & NA & 0.451 & 0.594 \\
\hline Saturated model & & 1 & 1 \\
\hline Independence model & & 0 & 0 \\
\hline
\end{tabular}

None of the criteria (NFI and CFI) are at a level to suggest that the model is a good fit

Table 38 Summary Table for Perceived Organizational Support and Cultural Values - Middle East

\begin{tabular}{|l|c|}
\hline & r \\
\hline Perceived Organizational Support and Individual Index (IDV) - 2a & -0.27 \\
\hline Perceived Organizational Support and Power Index (PDI) - 2b & -0.54 \\
\hline Perceived Organizational Support and Uncertainty Avoidance Index (UAI) - 2c & -0.42 \\
\hline Perceived Organizational Support and Masculinity Index (MAS) - 2d & 0.12 \\
\hline Perceived Organizational Support and Long-term Orientation Index (LTO) - 2e & 0.1 \\
\hline
\end{tabular}


There appears to be a significant negative relationship between perceived organizational support and the power distance (PDI) /uncertainty avoidance (UAI) indices. This does not support Hypothesis $2 \mathrm{~b}$ and $2 \mathrm{c}$. As the other correlations are not significant, Hypothesis $2 \mathrm{a}, 2 \mathrm{~d}$, and 2e are supported. The model used is not a good fit; the results for the Middle East region do not support Hypothesis 2.

Organizational Support and Cultural Values for North America are shown in Figure 9 and Table 39 and Table 40.

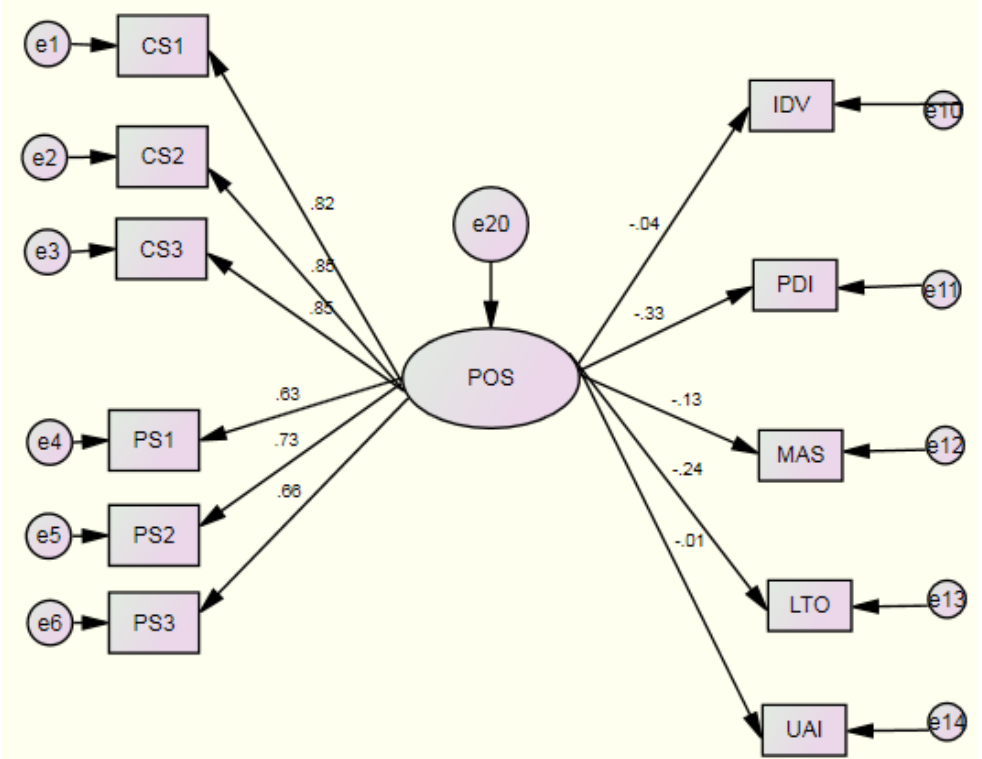

Figure 9 Structural Model of Perceived Organizational Support and Interest in Mentoring North America

Table 39 Model Fit Indices of Structural Model of Perceived Organizational Support and Cultural Values - North America

\begin{tabular}{|l|c|c|c|}
\hline \multicolumn{1}{|c|}{ Model } & GFI & NFI & CFI \\
\hline Default model & NA & 0.626 & 0.763 \\
\hline Saturated model & & 1 & 1 \\
\hline Independence model & & 0 & 0 \\
\hline
\end{tabular}


None of the criteria (NFI and CFI) are at a level to suggest that the model is a good fit.

Table 40 Summary Table for Perceived Organizational Support and Cultural Values - North America

\begin{tabular}{|l|c|}
\hline & r \\
\hline Perceived Organizational Support and Individual Index (IDV) - 2a & -0.04 \\
\hline Perceived Organizational Support and Power Index (PDI) - 2b & -0.33 \\
\hline Perceived Organizational Support and Uncertainty Avoidance Index (UAI) -2c & -0.01 \\
\hline Perceived Organizational Support and Masculinity Index (MAS) - 2d & -0.13 \\
\hline Perceived Organizational Support and Long-term Orientation Index (LTO) - 2e & -0.24 \\
\hline
\end{tabular}

There appears to be a significant negative relationship between perceived organizational support and the power distance (PDI) indices. This does not support Hypothesis 2b. As the other correlations are not significant, Hypothesis $2 \mathrm{a}, 2 \mathrm{c}, 2 \mathrm{~d}$, and $2 \mathrm{e}$ are supported. The model used is not a good fit; the results for the North America region do not support Hypothesis 2.

Organizational Support and Cultural Values for Russia \& Caspian are shown in Figure 10 and Table 41 and Table 42.

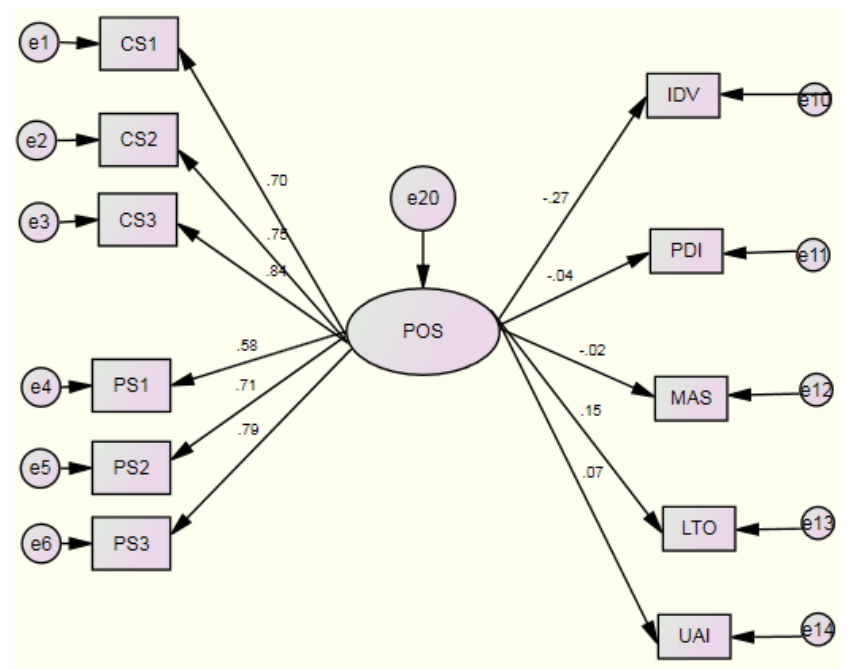

Figure 10 Structural Model of Perceived Organizational Support and Cultural Values - Russia \& Caspian 
Table 41 Model Fit Indices of Structural Model of Perceived Organizational Support and Cultural Values - Russia \& Caspian

\begin{tabular}{|l|c|c|c|}
\hline Model & GFI & NFI & CFI \\
\hline Default model & NA & 0.531 & 0.731 \\
\hline Saturated model & & 1 & 1 \\
\hline Independence model & & 0 & 0 \\
\hline
\end{tabular}

None of the criteria (NFI and CFI) are at a level to suggest that the model is a good fit.

Table 42 Summary Table for Perceived Organizational Support and Cultural Values - Russia \& Caspian

\begin{tabular}{|l|c|}
\hline & r \\
\hline Perceived Organizational Support and Individual Index (IDV) - 2a & -0.27 \\
\hline Perceived Organizational Support and Power Index (PDI) - 2b & -0.04 \\
\hline Perceived Organizational Support and Uncertainty Avoidance Index (UAI) - 2c & 0.07 \\
\hline Perceived Organizational Support and Masculinity Index (MAS) - 2d & -0.02 \\
\hline Perceived Organizational Support and Long-term Orientation Index (LTO) - 2e & 0.15 \\
\hline
\end{tabular}

There does not appear to be a significant relationship between perceived organizational support and the indices (highest being 0.27 ). Therefore hypothesis $2 \mathrm{a}, 2 \mathrm{~b}, 2 \mathrm{c}, 2 \mathrm{~d}$, and $2 \mathrm{e}$ are supported.

The model used is not a good fit; the results for the Russia \& Caspian region support Hypothesis 2.

Organizational Support and Cultural Values for Eastern Hemisphere are shown in Figure 11 and Table 43 and Table 44. 


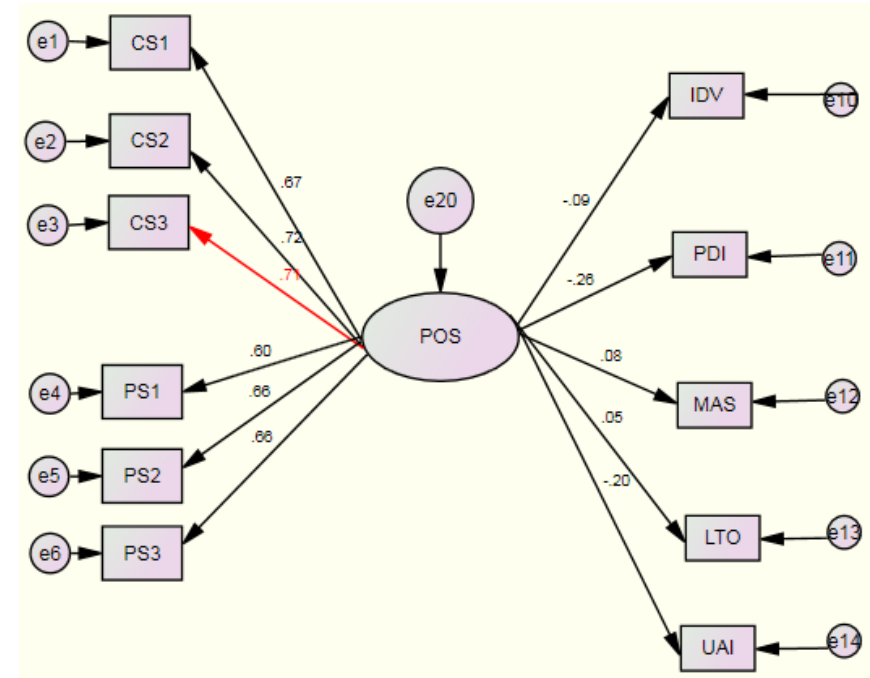

Figure 11 Structural Model of Perceived Organizational Support and Cultural Values Indices Eastern Hemisphere

Table 43 Model Fit Indices of Structural Model of Perceived Organizational Support and Cultural Values - Eastern Hemisphere

\begin{tabular}{|l|c|c|c|}
\hline Model & GFI & NFI & CFI \\
\hline Default model & NA & 0.621 & 0.666 \\
\hline Saturated model & & 1 & 1 \\
\hline Independence model & & 0 & 0 \\
\hline
\end{tabular}

None of the criteria (NFI and CFI) are at a level to suggest that the model is a good fit.

Table 44 Summary Table for Perceived Organizational Support and Cultural Values - Eastern Hemisphere

\begin{tabular}{|l|c|}
\hline & r \\
\hline Perceived Organizational Support and Individual Index (IDV) - 2a & -0.09 \\
\hline Perceived Organizational Support and Power Index (PDI) - 2b & -0.26 \\
\hline Perceived Organizational Support and Uncertainty Avoidance Index (UAI) - 2c & -0.2 \\
\hline Perceived Organizational Support and Masculinity Index (MAS) - 2d & 0.08 \\
\hline Perceived Organizational Support and Long-term Orientation Index (LTO) - 2e & 0.05 \\
\hline
\end{tabular}


There does not appear to be a significant relationship between perceived organizational support and the indices (highest being 0.26 ). Therefore hypothesis $2 \mathrm{a}, 2 \mathrm{~b}, 2 \mathrm{c}, 2 \mathrm{~d}$, and $2 \mathrm{e}$ are supported. The model used is not a good fit; the results for the Eastern Hemisphere support Hypothesis 2. Organizational Support and Cultural Values for Western Hemisphere are shown in Figure 12 and Table 45 and Table 46.

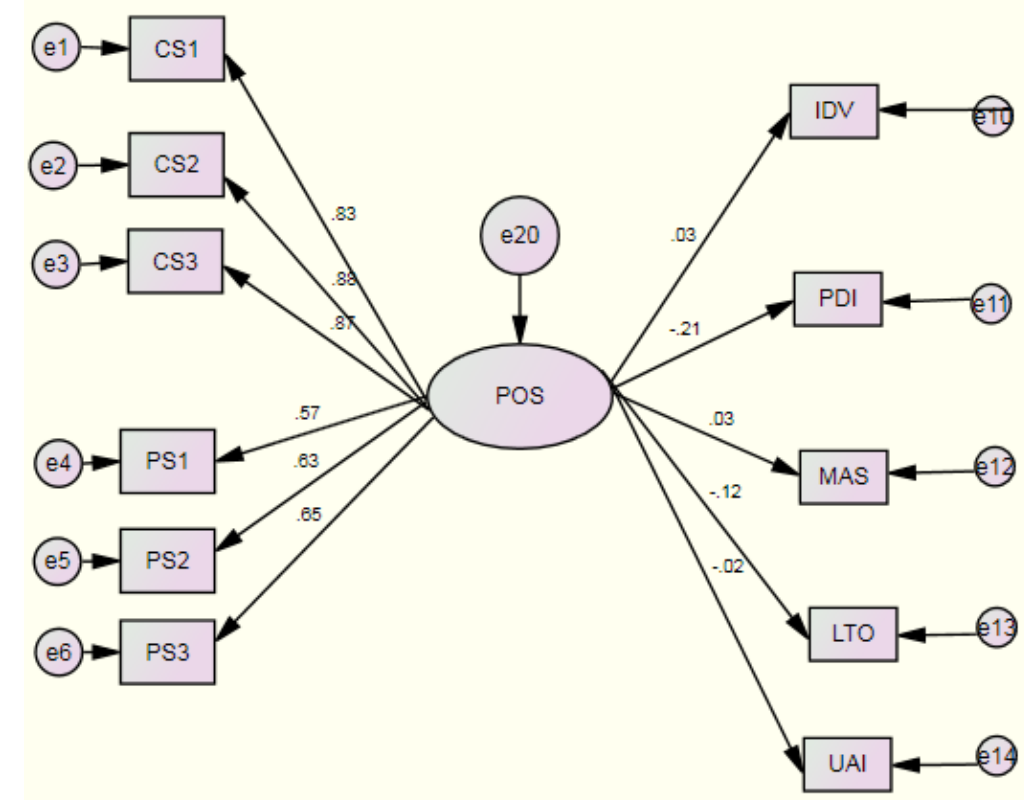

Figure 12 Structural Model of Perceived Organizational Support and Cultural Values Western Hemisphere

Table 45 Model Fit Indices of Structural Model of Perceived Organizational Support and Cultural Values - Western Hemisphere

\begin{tabular}{|l|c|c|c|}
\hline Model & GFI & NFI & CFI \\
\hline Default model & NA & 0.524 & 0.582 \\
\hline Saturated model & & 1 & 1 \\
\hline Independence model & & 0 & 0 \\
\hline
\end{tabular}


None of the criteria (NFI and CFI) are at a level to suggest that the model is a good fit.

Table 46 Summary Table for Perceived Organizational Support and Cultural Values Western Hemisphere

\begin{tabular}{|l|c|}
\hline & r \\
\hline Perceived Organizational Support and Individual Index (IDV) - 2a & 0.03 \\
\hline Perceived Organizational Support and Power Index (PDI) - 2b & -0.21 \\
\hline Perceived Organizational Support and Uncertainty Avoidance Index (UAI) - 2c & -0.02 \\
\hline Perceived Organizational Support and Masculinity Index (MAS) - 2d & 0.03 \\
\hline Perceived Organizational Support and Long-term Orientation Index (LTO) - 2e & -0.12 \\
\hline
\end{tabular}

There does not appear to be a significant relationship between perceived organizational support and the indices (highest being 0.21). Therefore Hypothesis $2 \mathrm{a}, 2 \mathrm{~b}, 2 \mathrm{c}, 2 \mathrm{~d}$, and 2e are supported. The model used is not a good fit; the results for the Western Hemisphere support Hypothesis 2.

\section{Research Hypothesis 3}

Employee perceived support can be used to accurately predict interest/ non interest in formal mentoring program participation. The mentoring questions are ordinal (yes/no) questions; as such, the coefficients were found using Path Analysis and Ordinal Regression for which a simple model was assumed. While the correlation found using Path Analysis is generally acceptable, not all publications accept Path Analysis when ordinals are involved; therefore, both tests have been included. Perceived Organizational Support and Intent to Participate in Mentoring are shown in Figures 13, 14, and 15 and Tables 47, 48, 49 and 50. 


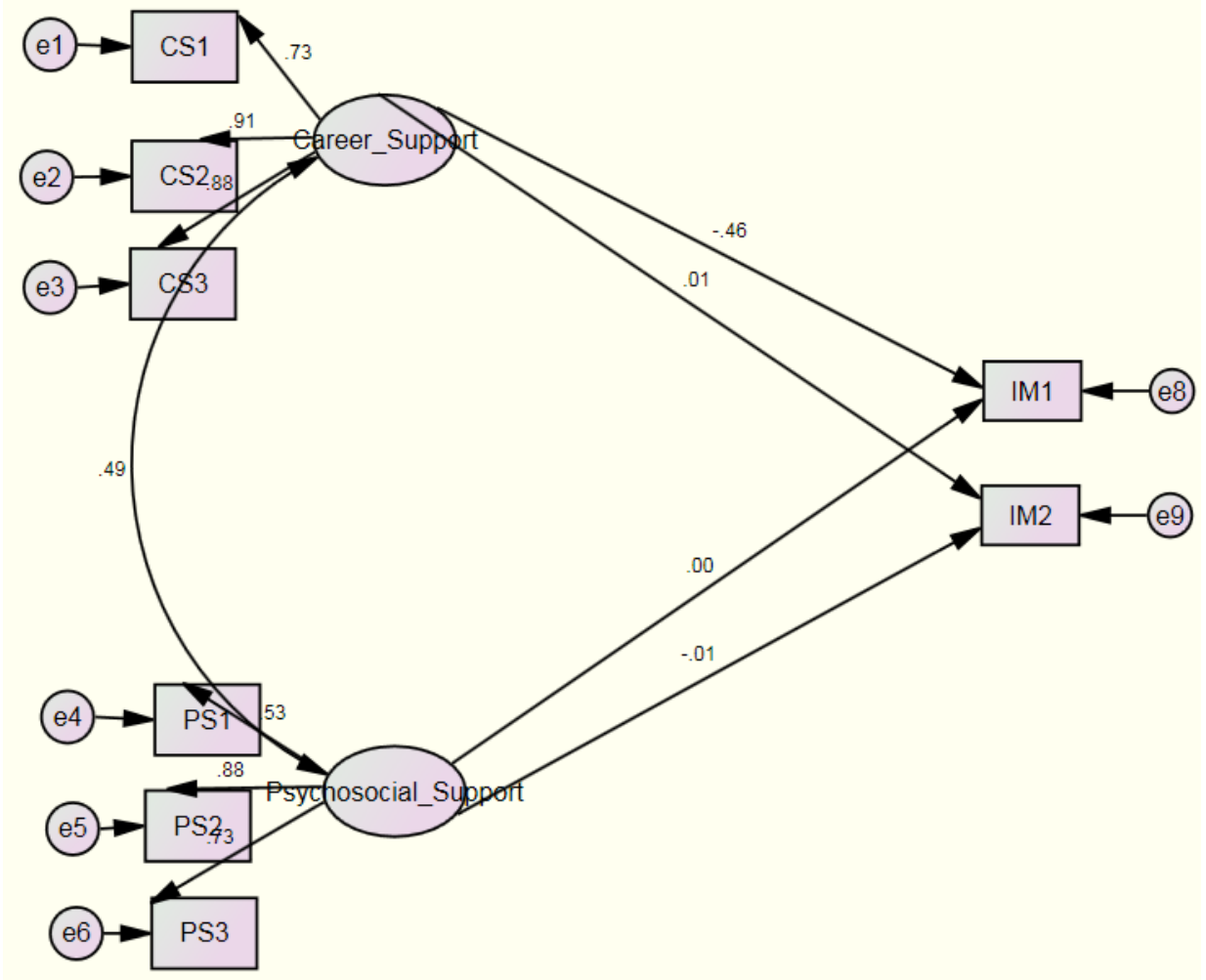

Figure 13 Structural Model of Perceived Organizational Support and Intent to Participate in Mentoring

Table 47 Model Fit Indices of Structural Model of Perceived Organizational Support and Intent to Participate in Mentoring

\begin{tabular}{|l|c|c|c|}
\hline \multicolumn{1}{|c|}{ Model } & GFI & NFI & CFI \\
\hline Default model & 0.973 & 0.961 & 0.977 \\
\hline Saturated model & 1 & 1 & 1 \\
\hline Independence model & 0.518 & 0 & 0 \\
\hline
\end{tabular}

The model has a GFI of 0.973 , a NFI of 0.961 and a CFI of 0.977 ; thus satisfying all three conditions; the model tested is a good fit. 
Table 48 Summary Table for Perceived Organizational Support and Intent to Participate in Mentoring

\begin{tabular}{|l|c|c|c|c|c|c|}
\hline & \multicolumn{2}{|c|}{$\begin{array}{c}\text { Path } \\
\text { Analysis }\end{array}$} & \multicolumn{2}{c|}{$\begin{array}{c}\text { Ordinal } \\
\text { Regression }\end{array}$} & \multicolumn{2}{c|}{$\begin{array}{c}\text { High Levels } \\
\text { Ordinal }\end{array}$} \\
\cline { 2 - 7 } & $\mathrm{R}$ & $\mathrm{r}^{2}$ & $\begin{array}{c}\text { Nagelkerke } \\
|\mathrm{r}|\end{array}$ & $\begin{array}{c}\text { Nagelkerke } \\
\mathrm{r}^{2}\end{array}$ & $\begin{array}{c}\text { Nagelkerke } \\
|\mathrm{r}|\end{array}$ & $\begin{array}{c}\text { Nagelkerke } \\
\mathrm{r}^{2}\end{array}$ \\
\hline $\begin{array}{l}\text { Career Support } \\
\text { and Interest in } \\
\text { Mentoring 1 -3a }\end{array}$ & -0.46 & 0.212 & 0.52 & 0.27 & 0.25 & 0.062 \\
\hline $\begin{array}{l}\text { Career Support } \\
\text { and Interest in } \\
\text { Mentoring 2-3c }\end{array}$ & 0.01 & 0.0001 & 0.369 & 0.136 & 0.167 & 0.028 \\
\hline $\begin{array}{l}\text { Psychosocial } \\
\text { Support and } \\
\begin{array}{l}\text { Interest in } \\
\text { Mentoring 1-3b }\end{array}\end{array}$ & 0 & 0 & 0.385 & 0.148 & 0.349 & 0.122 \\
\hline $\begin{array}{l}\text { Psychosocial } \\
\text { Support and } \\
\text { Interest in } \\
\text { Mentoring 2-3d }\end{array}$ & -0.01 & 0.0001 & 0.361 & 0.13 & 0.084 & 0.007 \\
\hline
\end{tabular}

The correlation between career support and interest in formal mentoring 1 was both significant (from Nagelkerke $|\mathrm{R}|$ ) and negative (from the path analysis), as predicted in Hypothesis 3a. It was also significant in regards to interest in formal mentoring 2 and positive. However, Hypothesis 3a predicted a negative relationship; as such, it is not supported in regards to interest in formal mentoring 2. In terms of high levels of career support the correlation to both interest in formal mentoring 1 and 2, the correlation wasn't significant (the highest correlation being 0.250). This supports Hypothesis $3 \mathrm{c}$.

The correlation between psychosocial support and interest in formal mentoring 1 is significant; however, whether it is positive or negative is undetermined. Therefore, in regards to interest in formal mentoring 1 , Hypothesis $3 b$ is not supported. In terms of interest in mentoring 2, which is both significant and negative, Hypothesis $3 \mathrm{~b}$ is supported. 
High level of psychosocial support actually had a significant correlation to interest in mentoring 1 . This was contrary to Hypothesis $3 \mathrm{~d}$. Therefore Hypothesis $3 \mathrm{~d}$ is not supported in regards to interest in mentoring 1 . In regards it interest in mentoring 2 , the correlation was not significant which supports Hypothesis $3 \mathrm{~d}$.

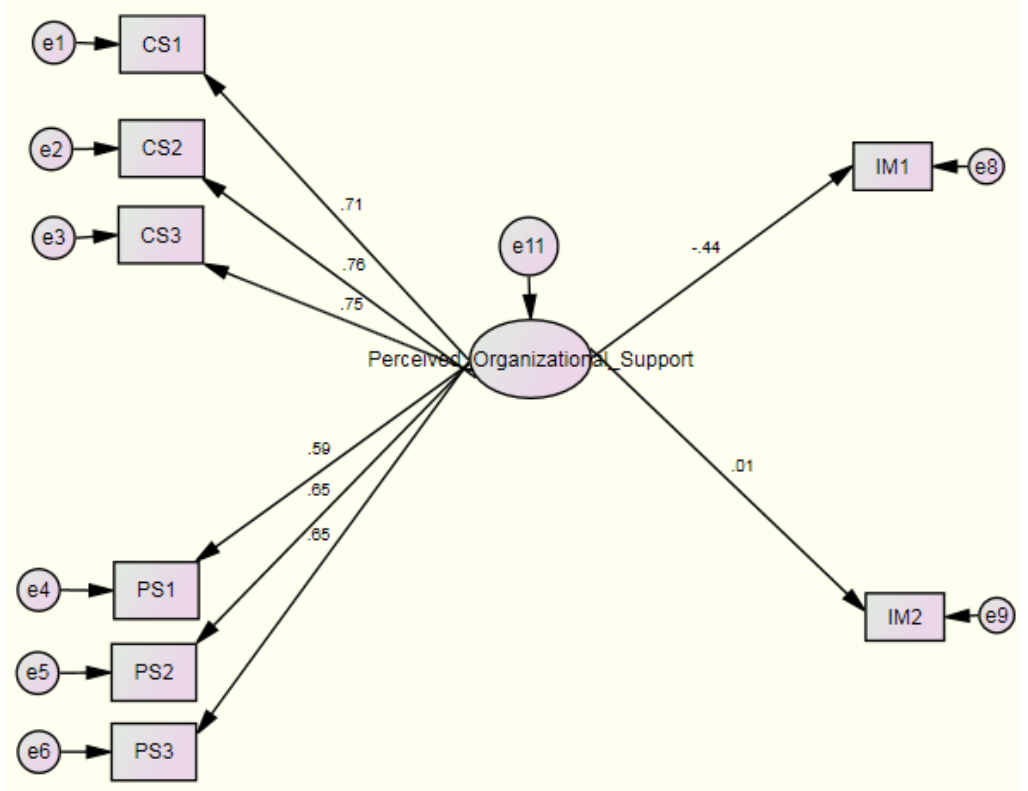

Figure 14 Structural Model of Perceived Organizational Support and Intent to Participate in Mentoring

Table 49 Model Fit Indices of Structural Model of Perceived Organizational Support and Intent to Participate in Mentoring

\begin{tabular}{|l|c|c|c|}
\hline \multicolumn{1}{|c|}{ Model } & GFI & NFI & CFI \\
\hline Default model & 0.754 & 0.653 & 0.668 \\
\hline Saturated model & 1 & 1 & 1 \\
\hline Independence model & 0.518 & 0 & 0 \\
\hline
\end{tabular}


Based upon Figure 14, the Structural Model of Perceived Organizational Support and Interest in Mentoring, Hypothesis 3, that perceived organizational support can be used to predict interest/non-interest in formal mentoring, is not completely supported. In the case of interest in formal mentoring 1 , there is support as there is a significant negative correlation. For interest in formal mentoring 2, there is not a significant relationship. Therefore, Hypothesis 3 is supported for interest in formal mentoring 1 but not for interest in formal mentoring 2 . However this model is weak, as evidenced by GFI, NFI, and CFI all being below 0.9 .

The conclusion that Hypothesis 3 lacks support is substantiated by perceived organizational support as a predictor to be supported; both career support and psychosocial would have to be useful predictors. This is unfortunately not the case.

\section{Research Hypothesis 4}

Employee perceived support is positively related to affective reactions.

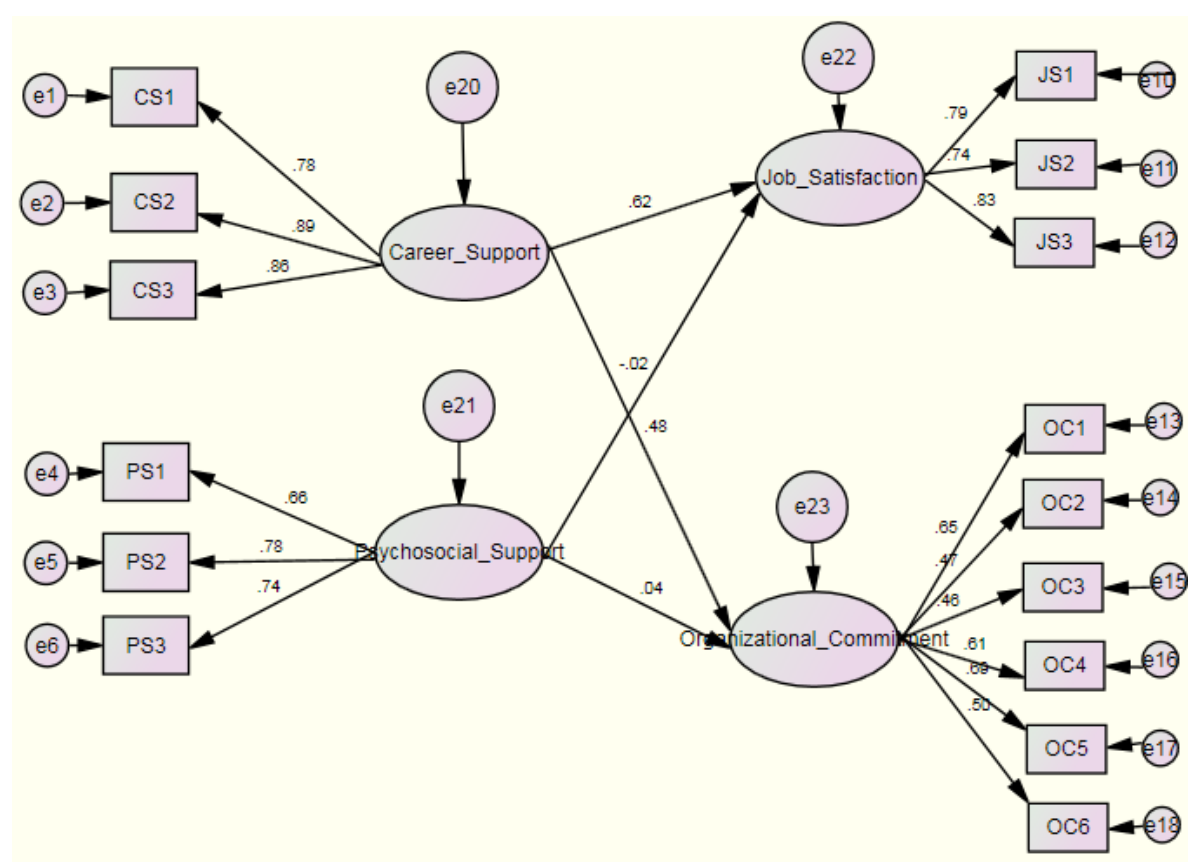

Figure 15 Structural Model of Job Satisfaction and Organizational Commitment to Interest in Mentoring 
Table 50 Model Fit Indices of Structural Model of Job Satisfaction and Organizational Commitment and Intent to Participate in Mentoring

\begin{tabular}{|l|c|c|c|}
\hline \multicolumn{1}{|c|}{ Model } & GFI & NFI & CFI \\
\hline Default model & 0.865 & 0.798 & 0.836 \\
\hline Saturated model & 1 & 1 & 1 \\
\hline Independence model & 0.407 & 0 & 0 \\
\hline
\end{tabular}

A GFI of 0.865 , a NFI is 0.798 , and a CFI of 0.836 , thus satisfying only one of three conditions suggests the model tested is a not a good fit.

Table 51 Summary Table for Career Support and Psychosocial Support to Job Satisfaction and Organizational Commitment

\begin{tabular}{|l|c|}
\hline & r \\
\hline Career Support and Job Satisfaction $-4 \mathrm{a}$ & 0.62 \\
\hline Career Support and Organizational Commitment $-4 \mathrm{c}$ & 0.48 \\
\hline Psychosocial Support and Job Satisfaction $-4 \mathrm{~b}$ & -0.02 \\
\hline Psychosocial Support and Organizational Commitment $-4 \mathrm{~d}$ & 0.04 \\
\hline
\end{tabular}

A significant positive relationship is evident between career support and job satisfaction with correlations of 0.62 and 0.48 . This supports Hypothesis $4 \mathrm{a}$ and $4 \mathrm{c}$. The relationship of psychosocial support to job satisfaction and organizational commitment is not significant as the correlations were -0.02 and 0.04 . This does not support Hypothesis $4 \mathrm{~b}$ and $4 \mathrm{~d}$. Perceived Organizational Support and Affective Reaction are shown in Figure 16 and Table 52 and Table 53. 


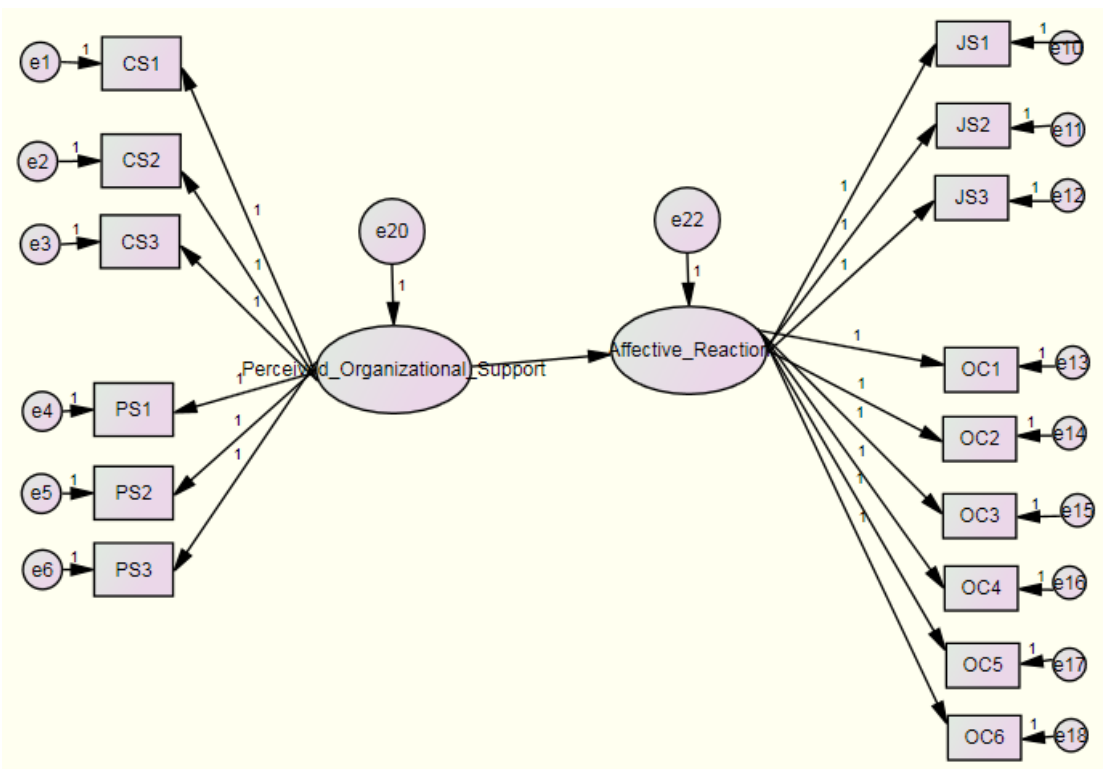

Figure 16 Structural Model of Perceived Organizational Support and Affective Reaction

Table 52 Model Fit Indices of Structural Model of Perceived Organizational Support and Affective Reaction

\begin{tabular}{|l|c|c|c|}
\hline \multicolumn{1}{|c|}{ Model } & GFI & NFI & CFI \\
\hline Default model & 0.705 & 0.612 & 0.643 \\
\hline Saturated model & 1 & 1 & 1 \\
\hline Independence model & 0.407 & 0 & 0 \\
\hline
\end{tabular}

With a GFI of 0.705 , a NFI of 0.612 , and a CFI of 0.643 , none of the three suggested conditions are satisfied suggesting the model tested is a not a good fit.

Table 53 Summary Table for Perceived Organizational Support to Affective Reaction

\begin{tabular}{|c|c|}
\hline & $\mathrm{r}$ \\
\hline Perceived Organizational Support and Affective Reaction & 0.49 \\
\hline
\end{tabular}


The model to test for a relationship between perceived organizational support and affective reaction is not a good model. The model does support Hypothesis 4 - perceived support is positively related to affective reactions. Results of Hypothesis 4 analyzed by region and hemisphere are in Appendix A-8 and A-9.

\section{Research Hypothesis 5}

Affective reactions of oilfield services employees can be used to accurately predict participation in a formal mentoring program. Job Satisfaction and Organizational Commitment and Intent to Participate in Mentoring are shown Figure 17 and Table 54.

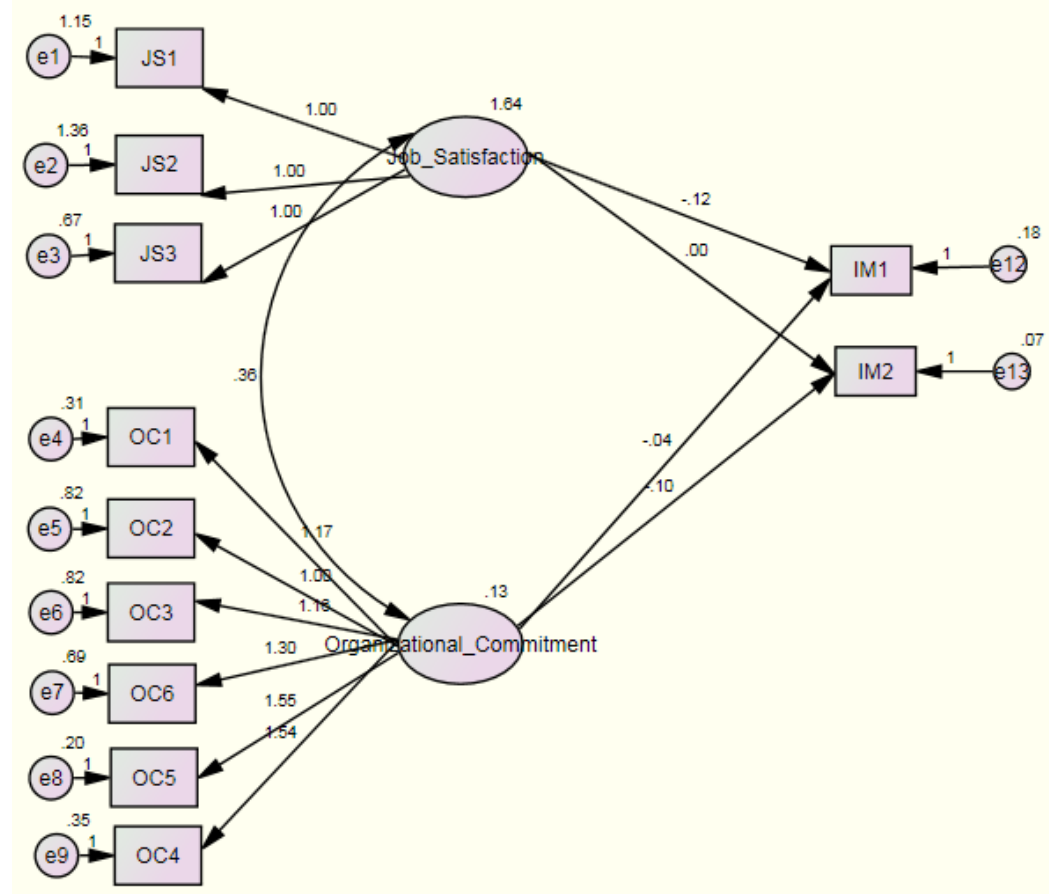

Figure 17 Structural Model of Job Satisfaction and Organizational Commitment to Intent to Participate in Mentoring 
Table 54 Model Fit Indices of Structural Model of Job Satisfaction and Organizational Commitment and Intent to Participate in Mentoring

\begin{tabular}{|l|c|c|c|}
\hline \multicolumn{1}{|c|}{ Model } & GFI & NFI & CFI \\
\hline Default model & 0.954 & 0.915 & 0.953 \\
\hline Saturated model & 1 & 1 & 1 \\
\hline Independence model & 0.498 & 0 & 0 \\
\hline
\end{tabular}

Satisfying all three conditions with a GFI of 0.954 , a NFI of 0.915 , and a CFI of 0.953 suggests the model tested is a good fit.

Affective Reaction and Intent to Participate in Mentoring are shown Figure 18 and Table

55.

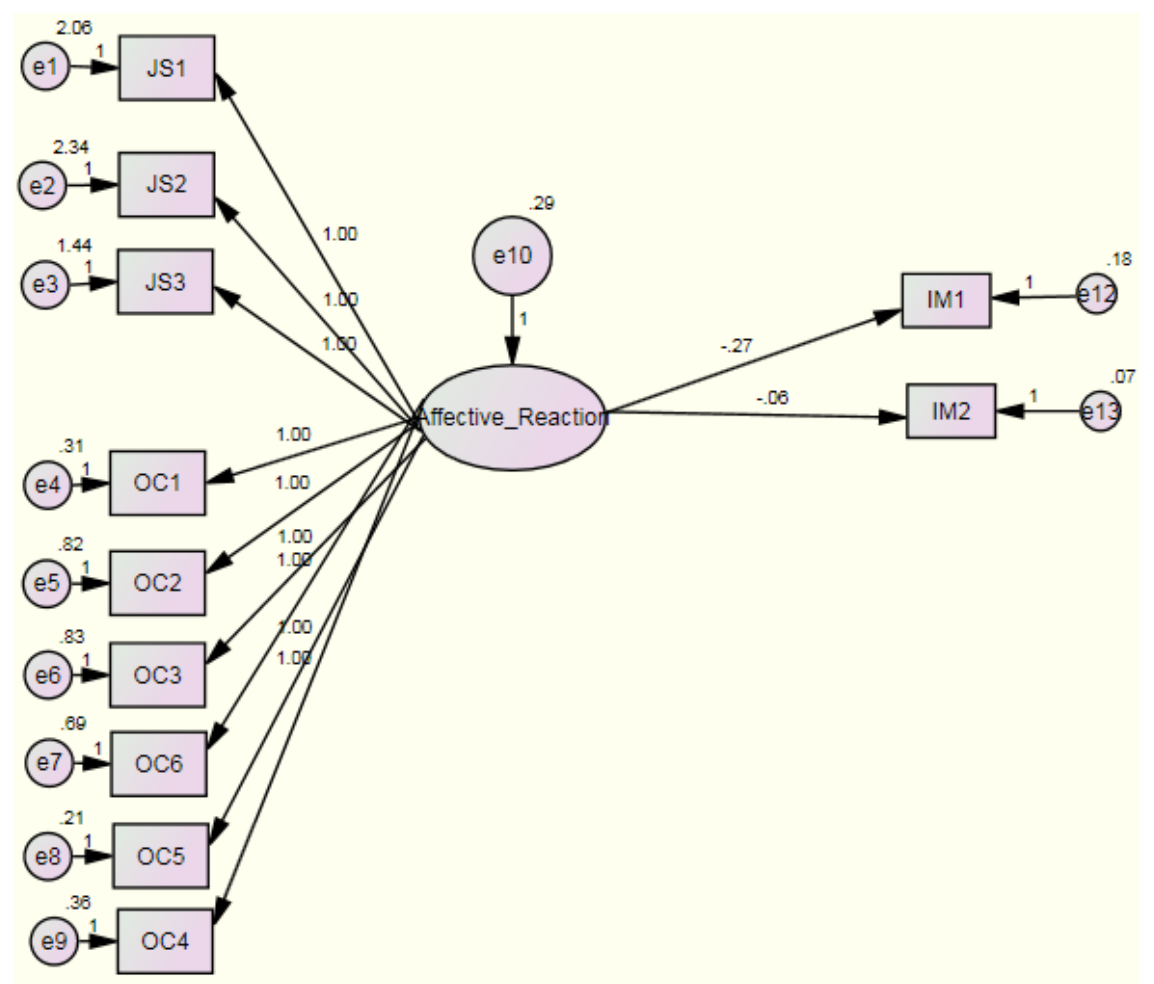

Figure 18 Structural Model of Affective Reaction and Intent to Participate in Mentoring 
Table 55 Model Fit Indices of Structural Model of Affective Reaction and Intent to Participate in Mentoring

\begin{tabular}{|l|c|c|c|}
\hline \multicolumn{1}{|c|}{ Model } & GFI & NFI & CFI \\
\hline Default model & 0.803 & 0.62 & 0.651 \\
\hline Saturated model & 1 & 1 & 1 \\
\hline Independence model & 0.498 & 0 & 0 \\
\hline
\end{tabular}

With a GFI of 0.803 , a NFI of 0.62 , and a CFI of 0.651 , none of the three conditions have been met, meaning the model tested is not a good fit.

Table 56 Summary Table for Affective Reaction and Intent to Participate in Mentoring

\begin{tabular}{|l|c|c|c|c|c|c|}
\hline & \multicolumn{2}{|c|}{$\begin{array}{c}\text { Path } \\
\text { Analysis }\end{array}$} & \multicolumn{2}{c|}{$\begin{array}{c}\text { Ordinal } \\
\text { Regression }\end{array}$} & \multicolumn{2}{c|}{$\begin{array}{c}\text { High Levels } \\
\text { Ordinal }\end{array}$} \\
\cline { 2 - 7 } & $\mathrm{r}$ & $\mathrm{r}^{2}$ & Nagelkerke & Nagelkerke & Nagelkerke & Nagelkerke \\
\cline { 3 - 7 } & -0.12 & 0.014 & 0.421 & 0.177 & 0.349 & 0.122 \\
\hline $\begin{array}{l}\text { Job Satisfaction and } \\
\text { Interest in } \\
\text { Mentoring 1 -5a }\end{array}$ & & 0 & 0.327 & 0.107 & 0.894 & 0.8 \\
\hline $\begin{array}{l}\text { Job Satisfaction and } \\
\text { Interest in } \\
\text { Mentoring 2 -5c }\end{array}$ & 0 & & & & & $\mathrm{r}^{2}$ \\
\hline $\begin{array}{l}\text { Organizational } \\
\text { Commitment and } \\
\text { Interest in } \\
\text { Mentoring 1 - 5b }\end{array}$ & -0.04 & 0.002 & 0.411 & 0.169 & 0.365 & 0.133 \\
\hline $\begin{array}{l}\text { Organizational } \\
\text { Commitment and } \\
\text { Interest in } \\
\text { Mentoring 1 - 5d }\end{array}$ & -0.1 & 0.01 & 0.391 & 0.153 & 0.415 & 0.172 \\
\hline $\begin{array}{l}\text { Affective Reaction } \\
\text { and Interest in } \\
\text { Mentoring 1 }\end{array}$ & -0.27 & 0.073 & 0.519 & 0.269 & 0.459 & 0.211 \\
\hline $\begin{array}{l}\text { Affective Reaction } \\
\text { and Interest in } \\
\text { Mentoring 2 }\end{array}$ & -0.06 & 0.004 & 0.49 & 0.24 & 0.449 & 0.202 \\
\hline
\end{tabular}

With Nagelkerke correlations ranging from 0.327 to 0.519 , a significant correlation is evident between job satisfaction, organizational commitment, and affective reaction and an interest in 
formal mentoring. Based upon the path analysis, this correlation appears to be uniformly negative. With the exception of job satisfaction to an interest in mentoring 2 which is undetermined.

As such, Hypothesis $5 \mathrm{a}$ and $5 \mathrm{~b}$ are unsupported, as they predicted a positive relationship with interest in formal mentoring. Hypothesis $5 \mathrm{c}$ and $5 \mathrm{~d}$ are supported, as they predicted a negative relationship with interest in formal mentoring. A possible exception may be job satisfaction to interest in mentoring 2; however, with a correlation of 0 from the path analysis, it was difficult to determine if the relationship was negative or not. Hypothesis 5 - affective reaction could be used to predict an interest in formal mentoring - is supported, as the Nagelkerke correlations were 0.519 and 0.490 .

\section{Summary}

The results from the factor analysis, correlation analysis, regression analysis, and path analysis provide some very useful insights about the relationships among the variables involved in the study. A more detailed discussion of the results, the implications for HRD research and practice, and recommendations for future research will be presented in Chapter $\mathrm{V}$ that follows. 


\section{CHAPTER V}

\section{DISCUSSION, CONCLUSIONS AND RECOMMENDATIONS}

There are four major sections in this chapter. In the first section, the research hypotheses and related findings are discussed. In the second section, the conclusion and limitations of the study are provided. In the third section, the implication of the current study for HRD research and practice is discussed. In the fourth and final section, recommendations and directions for future research are provided.

\section{Discussion}

The main research question of this study was concerned with relationships among cultural values, perceived organizational support (career and psychosocial), affective reaction (job satisfaction and organizational commitment) and the intent of Field Engineers - Level 1 (FE1s) to participate in a formal mentoring program in an oilfield services corporation. This research question was described by twenty-two research hypotheses. In the following sections, results concerning the research question and each of the hypotheses will be discussed.

Generally, employees' cultural values did not differ significantly across Hofstede's (1980) five cultural dimensions. Moreover, the relationship between cultural values and perceived organizational support was unrelated, that is, the correlation between cultural values and perceived organizational support was weak; however, when the relationship between the individual cultural values and perceived organization support was analyzed by region, Asia Pacific, Europe, Middle East, North America, and Russia \& Caspian show significant relationships. Furthermore, when analyzed by Hemisphere, results for the Eastern Hemisphere show no relationship, whereas, the Western Hemisphere show significant relationships. 
In examining the relationship between perceived organizational support and interest in formal mentoring, a significant relationship could not be found; however, the relationship between career support and interest in formal mentoring was significant as was psychosocial support. In examining the relationship between affective reaction and interest in formal mentoring, a significant relationship was found; moreover, when analyzed separately, both job satisfaction and organizational commitment correlated negatively with interest in mentoring.

In examining the relationship between perceived organizational support and affective reaction, a significant positive relationship was found. Although, a significant positive relationship is evident between career support and both job satisfaction and organizational commitment, no significant correlation could be found between psychosocial support and either job satisfaction or organizational commitment.

In the following sub-sections, the hypotheses of the study and results pertaining to each of the hypothesis are discussed. It is important to note several of the sub-hypotheses did not support the hypothesized relationships.

\section{Hypothesis 1}

According to Hypothesis 1, employees' cultural values will significantly differ among employees in an oilfield services corporation - across Hofstede's (1980) five cultural dimensions was not supported. In contrast to Hofstede's findings regarding cultural differences in his research, the data set analyzed in this study via ANOVA tests showed the only indices that had a significant difference are MAS by region and UAI by hemisphere. Furthermore, the data set analyzed using the Welch tests only showed a significant difference for LTO and UAI by hemisphere. Although many reasons could be attributed to the lack of significant difference, first the researcher would like to point out that neither the component variable, nor Hofstede's indices 
are normally distributed. Second, although the respondents were from forty-two different countries, all of the respondents were of similarity with regard to employee level, education level, and of course, all employees were hired and worked for the same organization. Lastly, and most importantly, the lack of numerous responses per country (only 1 respondent in some cases) introduced an un-equal bias in the responses as can be seen in Table 2. Additionally, the small sample size per country and, and in some cases, per region did not meet the minimal cases required for clustering data sets in order to run the more informative tests.

\section{Hypothesis 2}

According to Hypothesis 2, employees' individual cultural values - as framed by Hofstede (1980) - will be unrelated to their perceptions of support from their organization. The highest correlation was 0.25 ; as such, Hypothesis 2 was supported as a non-significant relationship between perceived organizational support and the individual cultural values was obtained. The same is true when Hypothesis 2 - analyzed by Hemisphere - both Eastern and Western Hemispheres did not show a significant relationship between perceived organizational support and the individual cultural values. When analyzed by region, Africa, Europe, Middle East and Russia \& Caspian support Hypothesis 2, as a non-significant relationship between perceived organizational support and the individual cultural values was obtained. Needless to say, Asia Pacific, Latin America and North America do not support Hypothesis 2, as a significant relationship between perceived organizational support and the individual cultural values was found. The relationship between the perceived organizational support factors (career support and psychosocial support) were analyzed and were found to support Hypothesis $2 \mathrm{a}-\mathrm{e}$. In fact, the highest correlation between career support and the individual cultural values was 0.18 (PDI) and the highest correlation between psychosocial support and the individual cultural 
values was 0.11 (IDV). The data was further analyzed by individual cultural value to perceived organizational support by Hemisphere and Region in Hypothesis 2a, 2b, 2c, 2d, and 2e. This was not expected. Per Hofstede's cultural studies, the cultural values would differ significantly in the study. In fact, the findings of this study are in direct opposition to Gerhart and Fang (2005) suggestion that culture due to region would be greater than organizational culture. This result is useful because the organizational culture seems to override the individual cultural values; thus speaking highly of the organization's ability to hire employees with like mind-sets, and further train and cultivate employees such that the employees have similar perceptions.

\section{Hypothesis $2 a$}

According to Hypothesis 2a, there will be no significant relationship between employee individualism and their perception of support from their organization. Eastern Hemisphere correlation was 0.09 ; Western Hemisphere correlation was 0.03 ; as such, Hypothesis 2a analyzed by hemisphere is supported - no relationship exists between employee individualism (IDV) and their perception of support from their organization. Analyzed by region, the following correlations between employee individualism and their perception of support from their organization were found: Africa (0.03), Asia Pacific (0.12), Europe (0.02), Latin America (0.05), Middle East (0.27), North America (0.04), and Russia \& Caspian (0.27). Although Hypothesis 2a is supported for each region (no significant relationship), the Middle East does show the most difference between the regions with regard to employee individualism and their perception of support from their organization. The result was not expected and can be the result of the organizational culture climate and/or the data set. 


\section{Hypothesis $2 b$}

According to Hypothesis $2 \mathrm{~b}$, there will be no significant relationship between employee power distance and their perception of support from their organization. Eastern Hemisphere correlation was 0.26 ; Western Hemisphere correlation was 0.21 ; as such, Hypothesis $2 b$ analyzed by hemisphere is supported - no relationship exists between power distance (PDI) and their perception of support from their organization. Analyzed by region, the following correlations between power distance and their perception of support from their organization were found: Africa (0.00), Asia Pacific (0.28), Europe (0.18), Latin America (0.04), Middle East (0.54), North America (0.33), and Russia \& Caspian (0.04). Hypothesis $2 \mathrm{~b}$ is supported for Africa, Europe, Latin America, and Russia \& Caspian (no significant relationship). The result was not expected and can be the result of the organizational culture climate and/or the data set. Hypothesis $2 b$ is not supported for Asia Pacific, Middle East, and North America (a significant relationship exists); furthermore, the Middle East shows the most difference between the regions with regard to power distance and their perception of support from their organization. This result was expected, especially regarding power distance (the acceptance that power is unequally distributed within an organization).

Hypothesis $2 c$

According to Hypothesis 2c, there will be no significant relationship between employee uncertainty avoidance and their perception of support from their organization. Eastern Hemisphere correlation was 0.02 ; Western Hemisphere correlation was 0.20 ; as such, Hypothesis $2 \mathrm{c}$ analyzed by hemisphere is supported - no relationship exists between uncertainty avoidance (UAI) and their perception of support from their organization. The researcher would like to point out the strength of the difference between the Eastern and Western hemispheres. 
The uncertainty avoidance cultural value shows the strongest difference between the two hemispheres. Analyzed by region, the following correlations between employee uncertainty avoidance and their perception of support from their organization were found: Africa (0.02), Asia Pacific (0.28), Europe (0.18), Latin America (0.04), Middle East (0.42), North America (0.01), and Russia \& Caspian (0.07). Hypothesis 2c is supported for Africa, Europe, Latin America, North America, and Russia \& Caspian (no significant relationship). The result was not expected and can be the result of the organizational culture climate and/or the data set.

Hypothesis $2 \mathrm{~b}$ is not supported for Asia Pacific and the Middle East (a significant relationship exists); furthermore, the Middle East shows the most difference between the regions with regard to uncertainty avoidance and their perception of support from their organization. This result was expected, especially regarding uncertainty avoidance (the level to which individuals feel at risk in uncertain situations).

\section{Hypothesis $2 d$}

According to Hypothesis $2 \mathrm{~d}$, there will be no significant relationship between employee masculinity and their perception of support from their organization. Eastern Hemisphere correlation was 0.08; Western Hemisphere correlation was 0.03; as such, Hypothesis $2 \mathrm{~d}$ analyzed by hemisphere is supported - no relationship exists between masculinity (MAS) and their perception of support from their organization. Analyzed by region, the following correlations between masculinity and their perception of support from their organization were found: Africa (0.21), Asia Pacific (0.03), Europe (0.08), Latin America (0.12), Middle East (0.12), North America (0.13), and Russia \& Caspian (0.02). Although Hypothesis $2 \mathrm{~d}$ is supported for each region (no significant relationship), Africa does show the most difference between the regions with regard to masculinity and their perception of support from their 
organization. The result was not expected and can be the result of the organizational culture climate and/or the data set.

\section{Hypothesis $2 e$}

According to Hypothesis 2e, there will be no significant relationship between employee long term orientation and their perception of support from their organization. Eastern Hemisphere correlation was 0.05 ; Western Hemisphere correlation was 0.12 ; as such, Hypothesis 2e analyzed by hemisphere is supported - no relationship exists between long term orientation (LTO) and their perception of support from their organization. Analyzed by region, the following correlations between long term orientation and their perception of support from their organization were found: Africa (0.24), Asia Pacific (0.17), Europe (0.13), Latin America (0.02), Middle East (0.10), North America (0.24), and Russia \& Caspian (0.15). Although Hypothesis 2e is supported for each region (no significant relationship), Africa and North America show the most difference between the regions with regard to long term orientation and their perception of support from their organization. The result was not expected and can be the result of the organizational culture climate and/or the data set.

\section{Hypothesis 3}

According to Hypothesis 3, employee perceived support can be used to accurately predict interest/ non interest in formal mentoring program participation. Hypothesis 3 is both supported and unsupported. Results indicate support for interest in formal mentoring 1, but not for interest in formal mentoring 2 . So, respondents with perceived organizational support were more likely to be presently (IM1) participating in the formal mentoring program and less likely 
to have the intent to participate in a future formal mentoring program (IM2). These results were expected as literature links mentoring benefits with organizational support.

The researcher posits that the results for Hypothesis 3 and $3 \mathrm{a}-3 \mathrm{~d}$, are confusing as the questions posed to the respondents, although validated and estimated to be reliable, may have been confusing to the respondents. Furthermore, the researcher was informed that all respondents were currently involved in the formal mentoring program, and so, all answers to Are you currently participating in a mentoring relationship through the LEAD Program (i.e. have you been placed with a Training Engineer)? (IM1) should have been 'Yes'. The pilot study reflected all 'Yes' answers, and so, confusion was thought to be minimal and/or non-existent. The fact that several respondents chose 'No' indicated a problem with the verbiage in the questions and how the question was read and understood within the different countries.

In order to further clarify the type of support felt, perceived organizational support was analyzed further by constructs career support and psychosocial support.

\section{Hypothesis $3 a$}

According to Hypothesis 3a, there will be a significant negative relationship between employee perceived career support and interest in formal mentoring program participation. The correlation between career support and interest in formal mentoring 1 and formal mentoring 2 was significant. This was expected as the researcher assumed those employees with perceived career support would already be presently in the formal mentoring program and would show negative interest in future participation in the formal mentoring program as their career support needs were currently being met. 
Hypothesis $3 b$

According to Hypothesis $3 b$, there will be a significant negative relationship between employee perceived psychosocial support and interest in formal mentoring program participation. The correlation between perceived psychosocial support and interest in formal mentoring 1 was significant, but not significant regarding interest in formal mentoring 2 . This both supports and is contrary to Hypothesis $3 b$. The not significant result was expected as the researcher assumed those employees with perceived psychosocial support would show no interest in future formal mentoring as their psychosocial support needs were currently being met. With regard to interest in mentoring 1, the researcher suggests that the respondents did not understand the question; and therefore, those with perceived psychosocial support were currently involved in the formal mentoring program, thus answering yes to IM1. Whether the correlation was positive or negative could not be determined.

\section{Hypothesis $3 c$}

According to Hypothesis 3c, employees with high levels of perceived career support will show no interest in formal mentoring program participation. The correlation between high levels of perceived career support and interest in formal mentoring 1 and formal mentoring 2 was not significant, which supports Hypothesis 3c. This was expected as the researcher assumed those employees with a high level of perceived career support would show no interest as their career support needs were currently being met.

\section{Hypothesis $3 d$}

According to Hypothesis 3d, employees with high levels of perceived psychosocial support will show no interest in formal mentoring program participation. The correlation 
between high levels of perceived psychosocial support and interest in formal mentoring 1 was significant, but not significant regarding interest in formal mentoring 2 . This both supports and is contrary to Hypothesis $3 \mathrm{~d}$. The not significant result was expected as the researcher assumed those employees with a high level of perceived psychosocial support would show now interest as their psychosocial support needs were currently being met. With regard to interest in mentoring 1, the researcher suggests that the respondents did not understand the question; and therefore, those with high levels of psychosocial support were currently involved in the formal mentoring program, thus answering yes to IM1.

\section{Hypothesis 4}

According to Hypothesis 4, employee perceived support is positively related to affective reactions. Hypothesis 4 is supported as was expected, such that literature suggests that job satisfaction leads to organizational commitment, and that employees who feel supported (either career or psychosocial support benefits from involvement in mentoring) from their organization, have higher job satisfaction and organizational commitment (Noe, Greemberger \& Wang (2002); Ragins, Cotton \& Miller, 2000).

\section{Hypothesis $4 a$}

According to Hypothesis $4 \mathrm{a}$, there will be a significant positive relationship between employee perceived career support and employee job satisfaction. Hypothesis 4a is supported with a strong correlation of 0.62. This is expected as Burlew (1991) states "mentored employees tend to be more satisfied with their jobs, get faster promotions, and make higher salaries" (p. 213) - all of which are career support functions within mentoring. 
Hypothesis $4 b$

According to Hypothesis $4 \mathrm{~b}$, there will be a significant positive relationship between employee perceived psychosocial support and employee job satisfaction. Hypothesis $4 \mathrm{~b}$ is not supported. With a correlation of 0.02 , no relationship exists between perceived psychosocial support and employee job satisfaction. This was not expected as psychosocial support is a benefit of mentoring and literature suggests mentoring leads to greater employee job satisfaction (Ragins, Cotton, \& Miller, 2000).

\section{Hypothesis $4 c$}

According to Hypothesis 4c, there will be a significant positive relationship between employee perceived career support and employee organizational commitment. Hypothesis $4 \mathrm{c}$ is supported with a correlation of 0.48 . This is expected and corresponds with Godshalk and Sosik (2007), who advocated individuals with mentors (protégés) have "higher expectations for advancement, career and job satisfaction, career commitment, and intention to stay at their organizations" (p.163).

Hypothesis $4 d$

According to Hypothesis $4 \mathrm{~d}$, there will be a significant positive relationship between employee perceived psychosocial support and employee organizational commitment. Hypothesis $4 \mathrm{c}$ is not supported. With a correlation of 0.04 , no relationship exists between perceived psychosocial support and employee organizational commitment. This was not expected as psychosocial support is a benefit of mentoring and literature suggests mentoring leads to greater employee organizational commitment (Allen, 2007). 
Hypothesis 5

According to Hypothesis 5, affective reactions of oilfield services employees can be used to accurately predict participation in a formal mentoring program. Hypothesis 5 is supported - affective reactions can be used to predict participation. A significant correlation exists regarding affective reaction and interest in mentoring 1 and interest in mentoring 2; however, based upon the path analysis, the relationship appears negative. This is expected as respondents with high levels of affective reaction would feel their current needs being met; therefore, would have no interest in participation in a formal mentoring program, either at present (IM1) or in the future (IM2). The researcher posits that the results for Hypothesis 5 and $5 \mathrm{a}-5 \mathrm{~d}$, are confusing as the questions posed to the respondents, although validated and estimated to be reliable, may have been confusing to the respondents. Furthermore, the researcher was informed, prior to the study, that all respondents were currently involved in the formal mentoring program, and so, all answers to Are you currently participating in a mentoring relationship through the LEAD Program (i.e. have you been placed with a Training Engineer)? (IM1) should have been 'Yes'. The pilot study reflected all 'Yes' answers, and so, confusion was thought to be minimal and/or non-existent. The fact that several respondents chose 'No' indicated a problem with the verbiage in the questions and how the question was read and understood within the different countries.

\section{Hypothesis 5 a}

According to Hypothesis 5a, there will be a significant positive relationship between employee job satisfaction and interest in formal mentoring program participation. Hypothesis 5a is unsupported, such that, even though there is a significant relationship between employee job satisfaction and interest in formal mentoring program participation, the relationship is negative. 
This was expected as satisfied employees would be less likely to enter an event that they might feel would not add to their satisfaction.

\section{Hypothesis $5 b$}

According to Hypothesis $5 \mathrm{~b}$, there will be a significant positive relationship between employee organizational commitment and interest in formal mentoring program participation. Hypothesis $5 \mathrm{~b}$ is unsupported, such that, even though there is a significant relationship between employee organizational commitment and interest in formal mentoring program participation, the relationship is negative. This was expected as committed employees would be less likely to enter an event that they might feel would not add to their commitment.

\section{Hypothesis $5 c$}

According to Hypothesis 5c, employees with high levels of job satisfaction will show no interest in formal mentoring program participation. Hypothesis $5 \mathrm{c}$ is supported, such that, the relationship between job satisfaction and interest in formal mentoring program participation (future) is significant, but with a correlation of 0 , it is difficult to determine if the employee did or did not have interest. The significant relationship was expected and the correlation of 0 could be due to the respondents not understanding the wording of questions IM1 or IM2.

\section{Hypothesis $5 d$}

According to Hypothesis 5d, employees with high levels of organizational commitment will show no interest in formal mentoring program participation. Hypothesis $5 \mathrm{~d}$ is supported, such that, a negative relationship between organizational commitment and interest in formal mentoring program participation (future) exists. This was expected. 


\section{Models of the Study}

The study had hypothesized and tested 17 path models. While some of the specific paths in these models, such as the one between perceived organizational support and affective reaction were supported from theory and previous empirical research findings, some of the paths were not. For instance, there were no specific theories or empirical studies that supported the hypothesized relationship between cultural values and perceived organizational support, or between perceived organizational support and intent to participate in formal mentoring, or between affective reaction and intent to participate in formal mentoring. As such, while part of the model was confirmatory model testing, part of it was exploratory model testing. The results of the path analysis show that the models analyzing affective reaction to intent to participate informal mentoring as well as the models analyzing affective reaction to intent to participate informal mentoring are a good fit.

The results of the study indicate that intent to participate in formal mentoring can be predicted by an individuals' perceived organizational support and/or their affective reaction. Moreover, the models analyzing cultural values (from both a region and hemisphere perspective) and perceived organizational support are a good fit. The results of the study indicate that the cultural values differ in certain values, in certain regions, as was to be expected based upon prior empirical findings. The fact that the differences were not vast, or even shown in certain cases, could be due to the number of responses garnered per country/region/hemisphere. Moreover, the similarity of the respondents, regardless of cultural value, could also be influenced by the fact that all respondents were of the same employee status and level within the oilfield organization thus extremely similar within an organizational culture component. 


\section{Conclusions and Limitations}

The researcher examined the relationships between cultural values, perceived organizational support (career and psychosocial support), affective reaction (job satisfaction and organizational commitment), and the intent to participate in a formal mentoring program in an oilfield services company, using several different analysis techniques. This was done to crossexamine the relationships and to enhance the accuracy of the results. Both the direct and indirect relationships among the variables involved in the study were examined.

While some of these relationships, such as affective reaction and perceived organizational support, have been researched in the past, some other relationships, such as perceived organizational support and affective reaction being used to predict intent to participate in a formal mentoring program, have not been researched sufficiently. Moreover, the cultural values relationship to perceived organizational support have not been researched sufficiently; more importantly, cultural values indirect effect intent to participate in a formal mentoring program have not been researched at all. In this context, the study's findings are significant.

The study also had several theoretical and methodological strengths. The study used a large literature base for its theoretical framework. More than 220 articles were reviewed, with the researcher choosing only 81 of the most prominent and reputed articles in academic journals in the fields of HRD, Psychology, Management, Engineering, and Organizational Behavior to be used in this study. Methodologically, the study had several strengths. First, it was a field study done in an actual industry-setting. Second, the sample size was much larger than the required sample for the given population. Third, the respondents were very diverse in terms of their cultural make-up.

The study also had some limitations. The researcher used self-reported data which depends on perceptions of respondents. But this limitation is usually accepted because self- 
reported surveys are considered the most practical method of collecting data representing individual attitudes and behaviors. Secondly, aspects such as cultural values, perceived organizational support (career and psychosocial), and affective reactions (job satisfaction and organizational commitment) are hard to observe; hence collecting objective data may not be possible. Most researchers consider these attributes as those most easily obtained by asking the individuals themselves. The self-reported data added non-response bias, which, according to Gall, Borg, and Gall (1996), unaddressed non-response error can threaten "the external validity of a study, a researcher's ability to draw conclusions, generalize results, and make inferences to broader audiences is weekend" (a.c. Dooley \& Linder, 2003, p. 107). As such with the findings in this study, the researcher is unable to generalize the research findings to another population making this research a case study for the oilfield services organization involved.

Another limitation of the study is that the researcher failed to authenticate the respondents' true intent to participate in a formal mentoring program. The mentoring-specific questions created by the organization were difficult to understand and possibly introduced bias into the response due to the respondent not understanding the true nature of the question. Furthermore, the respondents in this study should have all been current formal mentoring participants within the company, and so, the question Are you currently participating in a mentoring relationship through the LEAD Program (i.e. have you been placed with a Training Engineer)? (IM1) need not have been asked - thus leading to some of the respondent confusion. Furthermore, although the respondents were diverse in terms of origin or current country of citizenship, the respondents were not diverse in terms of their employment. All respondents were from a specified position within the company - Field Engineer 1 (FE1), and so their number of years of experience on the job, the industry they worked in, and or, the different aspects of their 
job were almost identical. This could possibly have led to an overarching organizational cultural component that was not measured in this study.

\section{Implications for HRD Research and Practice}

Perceived organizational support (career and psychosocial support) and affective reaction (job satisfaction and organizational commitment) are among the most explored areas in organizational research. Although cultural values is a highly researched area in organizational and management research, further research is needed on how this important factor affects HRD activities; moreover, how cultural values relate specifically to perceived organizational support, and future researchers need to re-examine both, the direct and indirect impact cultural values and perceived organizational support have on an individuals' intent to participate in mentoring programs. The model tested in this study included cultural values, perceived organizational support (career and psychosocial support), affective reaction (job satisfaction and organizational commitment), and intent [interest/non-interest] to participate in formal mentoring programs. It might be useful to field-test this model with other samples. Multiple samples from all oilfield services employees within this same company, and from the same countries and/or cultures would provide very useful insights on how the other employees within the same organization perceive these relationships.

Furthermore, it might be useful to field-test this model in another industry with and/or without a similar mentoring program in order to better understand other industry and employee motivation on the intent to participate in a mentoring program. Organizations face the increasing impact of globalization, and according to Ozkalp, Kirel, Sungur and Ozdemir (2008), have to adapt and prepare for the new demands of conveying and creating knowledge, per se, "the recognition of mentoring as an important transfer mechanism for knowledge within 
organizations" (p.67). Perspectives from employees in other industries leading to further insights about intent to participate in mentoring programs would be useful.

Lastly, it might be useful to examine the relationship between perceived organizational support and affective reaction from all oilfield services employees within this same company, and from the same countries and/or cultures would provide very useful insights on how the other employees within the same organization perceive these relationships. Although numerous studies have been conducted focusing on the benefits of mentoring to job satisfaction and organizational commitment, the relationship found in the one subset (FE1s) of this organization leaves much to be desired in terms of the breadth of understanding of these factors within the oilfield services organization as a whole.

\section{Recommendations and Directions for Future Research}

According to Collin (2004), both private and public organizations are seeking to improve their performance, effectiveness and competitiveness by implementing new ways of working. Ahmed (2006) suggested there are numerous ways to improve operations in a complex industry such as that of the oilfield service industry, and posits three that are often "quoted in the literature: quantum leaps in using conventional and non-conventional technologies and innovations; better use of capital investments; and improved management of the human resource" (p.188)." Rowden (2002) concurred and stated "developing the human resources of a company would seem to be key to increasing production and closing the gap between the level of worker skill and present and future needs" (p.409-410). One of the key methods organizations use to develop their human resources is mentoring. The amount of prior research on mentoring and the benefits received from mentoring [(perceived organizational support and affective 
reactions (job satisfaction and organizational commitment)], attests these factors hold important outcomes for an organization.

Although measuring employees perceived organizational support on affective reaction and the desire to participate in a formal mentoring program in an oilfield services organization may offer some useful insights about an employees' intent to participate in the formal mentoring program, the researcher found that measuring intent could not be measured in isolation.

Moreover, it is necessary to consider factors in the workplace that could potentially impede or enhance the employee's intent to participate. In fact, there are many individual characteristics or personal factors, namely cultural values, which can affect the employee's intent to participate. This study did not focus specifically on how cultural values affect the employee's intent to participate, but rather attempted to better understand how cultural values had an influence on perceived organizational support as well as, the researcher sought to better understand the differences in cultural values felt by employees of the same organization, completing the same job, with the same job level expectations.

In this study, the researcher examined the correlational effects of an employee's desire to participate in a formal mentoring program in an oilfield services organization based upon their perceived organizational support and their affective reaction. The perceptions of 341 Field Engineer 1s (FE1s) were collected using a 44-item questionnaire. A series of analyses, including descriptive statistics, factor analysis, correlation analysis, regression analysis, path analysis, and structural equation modeling was done to test the hypotheses of the study. The results from the analyses suggested cultural values amongst the FE1s do not differ significantly; moreover, the cultural values do not affect the FE1s perceived organizational support. It was found that FE1's perceived organizational support (career and psychosocial support) and affective reactions (job satisfaction and organizational commitment) predicted the FE1s intent to participate in a formal 
mentoring program in the oilfield services company. The implications of this study to HRD research and practice were discussed. Further, recommendations for future research were made. 


\section{REFERENCES}

Adler, N. (2002). International Dimensions of Organizational Behavior. Cincinnati, OH: SouthWestern.

Agho, A.O., Mueller, C.W., \& Price, J.L. (1993). Determinants of employee job satisfaction: An empirical test of a causal model. Human Relations, 46(8), 1007-1020.

Ahmed, H. (2006). Improved operations through manpower management in the oil sector. Journal of Petroleum Science \& Engineering, 55, 187-199.

Alderfer, C., \& Thomas, D.A. (1988). The significance of race and ethnicity for understanding organizational behavior. In C. Cooper, \& I. T. Robertson, eds. International Review of Industrial and Organizational Psychology (pp. 1-42). London: John Wiley \& Sons.

Allen, D.G., Griffeth, R.W., \& Shore, L.M. (2003). The role of perceived organizational support and supportive human resource practices in the turnover process. Journal of Management, 29: 99-118.

Allen, T.D., Dobbins, G.H., Poteet, M.L., \& Russell, J.E. (1997). A field study of factors related to supervisors' willingness to mentor others. Journal of Vocational Behavior, 50, $1-22$.

Allen, T.D, Maetzke, S.B., \& Russell, J.A. (1997). Formal peer mentoring. Group \& Organization Management, 22(4), 488-507.

Allen, T.D., Poteet, M.L., \& Russell, J.E.A. (2000). Protégé selection by mentors: What makes the difference? Journal of Organizational Behavior, 271-282.

Allen, T.D., \& Eby, L.T. (2003). Relationship effectiveness for mentors: Factors associated with learning and quality. Journal of Management, 29, 469-486.

Allen, T.D., Eby, L.T., Lentz, E., Lima, L., \& Poteet, M.L. (2004). Mentoring benefits: A metaanalysis. Journal of Applied Psychology, 89, 127-136.

Allen, T.D., Eby, L.T., \& Lentz, E. (2006). Mentorship behaviors and mentorship quality associated with formal mentoring programs: Closing the gap between research and practice. Journal of Applied Psychology, 91(3), 567-578.

Allen, T.D. (2007). Mentoring relationships from the perspective of the mentor. In B. Ragins, \& K. E. Kram, eds. The Handbook of Mentoring at Work: Theory, Research, and Practice (pp. 123-147). Los Angeles, CA: Sage Publications.

Allen, T.D., \& Eby, L.T. (2007). Common bonds: An integrative perspective on mentoring. In T. D. Allen, \& L. T. Eby, eds. Blackwell Handbook of Mentoring (pp. 397-419). Oxford: Blackwell. 
Anthias, F. \& Yuval Davis, N. (1992). Racialised Boundaries. London: Routledge.

Arbuckle, J.L. (2010). IBM SPSS Amos 19 User's Guide. Chicago: Amos Development Corporation.

Armstrong, S., Allinson, C.W., \& Hayes, J. (2002). Formal mentoring systems: An examination of the effects of mentor/protege cognitive styles on the mentoring process. Journal of Management Studies, 39(8), 1111-1137.

Aryee, S., \& Chay, Y.W. (1994). An examination of the impact on career-oriented mentoring on work commitment attitudes and career satisfaction among professional and managerial employees: An interactionist approach. British Journal of Management, 5, 241-249.

Atkinson, D., Casas, A., \& Neville, H.(1991). The mentorship of ethnic minorities in professional psychology. Professional Psychology: Research and Practice, 22, 336-338.

Balfour, D.L., \& Wechsler, B. (1990). Organizational commitment: A reconceptualization and empirical test of public-private difference. Public Productivity \& Management Review, $10,355-367$.

Balfour, D.L., \& Wechsler, B. (1991). Commitment, performance, and productivity in public organizations. Public Productivity \& Management Review, 14, 355-367.

Barham, K. \& Conway, C. (1998), Developing business and people internationally: A mentoring approach. Berkhampstead: Ashridge Research.

Barney, J. (1999). Firm resources and sustained competitive advantage. Journal of Management, $17,99-120$.

Bass, B.M. (1990). Bass and Stodgill's handbook of leadership. New York, NY: Free Press.

Bateman, T.S., \& Strasser, S. (1984). A longitudinal analysis of the antecedent of organizational commitment. Academy of Management Journal, 27, 95-112.

Baugh, S., \& Fagenson-Eland, E. A. (2007). Formal mentoring programs. In B. Ragins, eds., The Handbook of Mentoring at Work: Theory, Practice, and Research (pp. 249-271). Los Angeles, CA: Sage Publications.

Baumeister, R.F., \& Leary, M.R. (1995). The need to belong: Desire for interpersonal attachments as fundamental human emotion. Psychological Bulletin, 117, 497-529.

Bearman, S., Blake-Beard, S.D., Crosby, F.J., \& Hunt, L. (2007). Future mentoring research: Cutting across mentoring themes and contexts. In T.D. Allen \& L.T. Eby, eds., Blackwell handbook of mentoring: A multiple perspectives approach. Malden, MA: Blackwell Publishing.

Bentler, P.M. \& Bonett, D.G. (1980). Significance tests and goodness of fit in the analysis of covariance structure. Psychological Bulletin, 88:588-606. 
Betts, S., \& Pepe, L.J. (2006). The perceived value of mentoring: Empirical development of a five-factor framework. Journal of Organizational Culture, Communication and Conflict, $10(2), 105-115$.

Blake-Beard, S., Murrell, A., \& Thomas, D. (2007). Unfinished business. In B.Ragins, eds., The Handbook of Mentoring at Work: Theory, Practice, and Research (pp. 223-247). Los Angeles, CA: Sage Publications.

Blau, P. (1964). Exchange and power in social life. New York, NY: Wiley.

Bouquillion, E.A., Lee, D.Y., \& Sosik,J.J. (2005). It's only a phase: Examining trust, identification, and mentoring functions received across the mentoring phases. Mentoring and Tutoring, 13, 239-258.

Bowen, D. (1982). On considering aspects of the mentoring process. Behavior Today, 13, 4-5.

Bowen, D. (1985). Were men meant to mentor women? Training and Development Journal, 39, 31-34.

Bowling, N., Beehr, T.A., \& Lepisto, L.R. (2006). Beyond job satisfaction: A five-year prospective analysis of the dispositional approach to job attitudes. Journal of Vocational Behavior, 69, 315-330.

Bowling, N., \& Hammond, G.D. (2008). A meta-analytic examination of the construct validity of the michigan organizational assessment questionnaire job satisfaction subscale. Journal of Vocational Behavior, 73, 63-77.

Bozionelos, N. (2002). Mentoring provided: Relation to mentor's career success, personality and mentoring received. Journal of Vocational Behavior, 64, 24-46.

Bragg, A. (1989). Is a mentor program in your future? Sales and Marketing Management, 141 (Sept.), 54-59.

Brooks, G. (2002). Knowledge-based structures and organizational commitment. Management Decision, 40(2), 556-573.

Budhwar, P.S., \& Sparrow, P.R. (2002). An integrative framework for understanding crossnational human resource management practices. Human Resource Management Review, $12,377-403$.

Burke, R. (1984). Mentors in organizations. Group \& Organizational Studies, 9(3), 353-372.

Burke, R., McKeen, C.A., \& Mckenna, C.S. (1993). Correlates of mentoring in organizations: The mentor's perspective. Psychological Reports, 67, 883-896.

Burlew, L. (1991). Multiple mentor model: A conceptual framework. Journal of Career Development, 17(3), 213-219. 
Cammann, C., Fichman, M., Jenkins, D., \& Klesh. (1983). Assessing the attitudes and perceptions of organizational members. In S. Seashore, E. Lawler, P. Mirvis, \& C. Cammann, eds., Assessing organizational change: A guide to methods, measures, and practices. John Wiley \& Sons.

Caproni, P. (2005). Managing Cultural Diversity (2nd ed.). Upper Saddle River, NJ: Pearson Prentice Hall.

Carmines, E.G. \& Zeller, R.A. (1979). Reliability and validity assessment: quantitative applications in the social sciences. Thousand Oaks, CA: Sage Publications, Inc.

Castro, S.L. \& Scandura, T.A. (2004, November 3-6). The tale of two measures: Evaluation and comparison of Scandura's (192) and Ragins and McFarlin's (1990) mentoring measures. Paper presented at the Southern Management Association Meeting. San Antonio, TX.

Chao, G. (1997). Mentoring phases and outcomes. Journal of Vocational Behavior, 51, 15-28.

Chao, G. (2007). Mentoring and organizational socialization. In B.Ragins, eds., The Handbook of Mentoring at Work: Theory, Practice, and Research (pp. 179-196). Los Angeles, CA: Sage Publications.

Chao, G.T., \& Moon, H. (2005). The cultural mosaic: A metatheory for understanding the complexity of culture. Journal of Applied Psychology, 90, 1128-1140.

Chen, Z.X., \& Francesco, A.M. (2000). Employee demography, organizational commitment, and turnover intentions in China: do cultural differences matter? Human Relations, 3(6), 869-887.

Claes, R., \& Ruiz-Quintanilla, S.A. (1998). Influences of early career experiences, occupational group, and national culture on proactive career behavior. Journal of Vocational Behavior, 52, 357-378.

Clutterbuck, D. \& Megginson, D. (1999). Mentoring executives and directors. Boston, MA: Butterworth Heinemann.

Clutterbuck, D. (2007). An international perspective on mentoring. In B.Ragins, eds., The Handbook of Mentoring at Work: Theory, Practice, and Research (pp. 633-655). Los Angeles, CA: Sage Publications.

Collin, K. (2004). The role of experience in work and learning among design engineers. International Journal of Training and Development, 8(2), 111-127.

Coolidge, F.L. (2006). Statistics: A gentle introduction (2ed). Thousand Oaks, CA: Sage Publications, Inc.

Cranny, C.J., Smith, C.P., \& Stone, E.F. (1992). Job Satisfaction: How people feel about their jobs and how it affects their performance. New York, NY: Lexington Books. 
DeLong, T., Gabarro, J.J., \& Lees, R.J. (2008). Why mentoring matters in a hypercompetitive world. Harvard Business Review, 115-121.

Dooley, L.M. \& Linder, J.R. (2003). The handling of nonresponse error. Human Resource Development Quarterly, 14:1, 99-110.

Donaldson, S.I., Ensher, E.A., \& Grant-Vallone, E.J. (2000). Longitudinal examinations of mentoring relationship on organizational commitment and citizenship behavior. Journal of Career Development, 26, 233-249.

Dougherty, T., \& Dreher, G.F. (2007). Mentoring and career outcomes. In B.Ragins, eds., The Handbook of Mentoring at Work: Theory, Practice, and Research (pp. 51-93). Los Angeles, CA: Sage Publications.

Douglas, C.A., \& McCauley, C.D. (1999). Formal developmental relationships: A survey of organizational practices. Human Resource Development Quarterly, 10, 203-220.

Dowling, P., Schuler, R.S., \& Welch, D.E. (1999). International Human Resource Management. Cincinnati, OH: South-Western.

Dreher, G.F., \& Ash, R.A. (1990). A comparative study of mentoring among men and women in managerial, professional, and technical positions. Journal of Organizational Behavior, $75,539-546$.

Drost, E., Frayne, C.A., Geringer, J.M., \& Lowe, K.B. (2002). Benchmarking training and development practices: A multi-country comparative analysis. Human Resource Management, 41(1), 67-86.

Eby, L. T. (1997). Alternative forms of mentoring in changing organizational environments: A Conceptual extension of the mentoring literature. Journal of Vocational Behavior, 51, $125-144$.

Eby, L., \& Lockwood, A. (2004). Proteges and mentors reactions to participating in formal mentoring programs: A qualitative investigation. Journal of Vocational Behavior, 67, 441-458.

Eby, L., Durley, J.R., Evans, S. C., \& Ragins, B.R. (2006). The relationship between short-term mentoring benefits and long-term mentor outcomes. Journal of Vocational Behavior, 69, 424-444.

Eby, L., Allen, T.D., Evans, S.C., DuBois, D.L., \& Ng, T.(2007). Does mentoring matter? A multidisciplinary meta-analysis comparing mentored and non-mentored individuals. Journal of Vocational Behavior, 72, 254-267.

Eddy, E., Alliger, G., D'Abate, C., Givens, S., \& Tannenbaum, S. (2001). Mentoring in industry: The top 10 issues when building and supporting a mentoring program. Technical report 
prepared for the Naval Air Warfare Training Systems Division (Contract No. N6133999-D-0012).

Egan, T., Bartlett, K.R., \& Yang, B. (2004). The effects of organizational learning culture and job satisfaction on motivation to transfer learning and turnover intention. Human Resource Development Quarterly, 15 (3), 279-301.

Egan, T., \& Rosser, M.H. (2004). Do formal mentoring programs matter?: A longitudinal randomized experimental study of women healthcare workers. 226-233. In Egan, T. M. \& Morris, L., eds., Proceedings of the 2004 Academy of Human Resource Development Conference (pp. 226-233). Austin, Texas: Academy of Human Resource Development.

Egan, T., \& Song, Z. (2008). Are facilitated mentoring programs beneficial? A randomized experimental field study. Journal of Vocational Behavior, 72, 351-362.

Ehrich, L.C., Hansford, B., \& Tennent, L. (2001). Closing the divide: Theory and practice in mentoring. ANZAM 2001 Conference, (pp. 5-7). Auckland, New Zealand.

Emery, C.R., \& Oertel, S. (2006). An examination of employee culture-based perceptions as predictor of motivation. Journal of Organizational Culture, Communication and Conflict, 10(2), 13-30.

Ensher, E.A., \& Murphy, S.E. (1997). Effects of race, gender, perceived similarity, and contact on mentor relationships. Journal of Vocational Behavior, 50, 460-481.

Fagenson, E. (1989). The mentor advantage: Perceived career/job expereinces of proteges versus non-proteges. Journal of Organizational Behavior, 10, 309-320.

Farylo, B., \& Paludi, M.A. (1985). Developmental discontinuities in mentor choice by male students. The Journal of Social Psychology, 125, 521-522.

Feldman, D.C., Folks, W.R., \& Turnley, W.H. (1999). Mentor-protege diversity and its impact on international internship experiences. Journal of Organizational Behavior, 20, 597611.

Fields, D.L. (1983). Taking the Measure of Work: A guide to validated scales for organizational research and diagnosis. Thousand Oaks, CA: Sage Publications.

Fullbright, K. (1985, Fall/Winter). The myth of the double advantage: Black female managers. Review of the Black Political Economy, 14(2-3), 33-45.

Gagne, M., \& Deci, E.L. (2005). Self-determination theory and work motivation. Journal of Organizational Behavior, 32, 331-363.

Gall, M. D., Borg, W R., \& Gall, J. P (1996). Educational research: An introduction (6th ed.). White Plains, NY: Longman. 
Gentry, W.A., Sadri, G., \& Weber, T.J.(2008). Examining career-related mentoring and managerial performance across cultures: A multilevel analysis. Journal of Vocational Behavior, 72, 241-253.

Gerhart, B., \& Fang, M. (2005). National culture and human resource management: assumptions and evidence. Interntional Journal of Human Resource Management, 16(6), 971-986.

Gibbons A. (2000). Training Journal, Getting the most from mentoring, p.18-20. Retrieved from: http://www.coachingnetwork.org.uk/resourcecentre/articles/ViewArticle.asp?artId=51

Gibb, S. \& Megginson, D. (1993). Inside corporate mentoring schemes: A new agenda of concerns. Personnel Review, (22:1), 40-54.

Godshalk, V.M., \& Sosik, J.J. (2007). Mentoring and Leadership. In B. Ragins, \& K.E. Kram, eds., The Handbook of Mentoring at Work: Theory, Research, and Practice (pp. 149178). Los Angeles, CA: Sage Publisher.

Gouillart, F. J. \& Kelly, J.N. (1995). Transforming the organization: Reframing corporate direction, restructuring the organization, revitalizing the enterprise, renewing people. New York: McGraw- Hill.

Hackman, J.R. \& Oldham, G.R. (1980). Work redesign. Reading, MA: Addison-Wesley.

Hagenow, N.R. \& McCrea, M.A. (1994). A mentoring relationship. Nursing Management 25(12), 42-43.

Halatin, T. (1981, February). Why be a mentor? Supervisory Management, 36-39.

Hall, D.T., Nygren, H.T., \& Schneider, B. (1970). Personal factors in organizational identification. Administrative Science Quarterly 15(2), 176-190.

Hamilton, E. (1942). Mythology. Boston, MA: Little, Brown \& Co.

Hartmann, L.C., \& Bambacas, M. (2000). Organizational commitment: A multi-method scale analysis and test of effects. The International Journal of Organizational Analysis, 8(1), $89-108$.

Hayes, H.B., Alagaraja, M. \& Dooley, L.M. (2003). Human capital theory: A reflection on retention of employees in the hospitality industry. Academy of Human Resource Development Proceedings, Minnesota.

Hegstad, C.D., \& Wentling, R.M. (2004). The development and maintenance of exemplary formal mentoring programs in Fortune 500 companies. Human Resource Development Quarterly, 15, 421-448.

Heimann, B., \& Pittenger, K.S. (1996). The impact of formal mentorship on socialization and commitment of newcomers. Journal of Management, 8(1), 108-117. 
Henle, C. (2005). Predicting workplace deviance from the interaction between organizational justice and personality. Journal of Managerial Issues, 77(2), 247-264.

Hinkin, T.R., \& Tracey, J.B. (2000). The Cost of Turnover. Putting a Price on the Learning Curve. Cornell Hotel and Restaurant Administration Quarterly, June, 14-22.

Hofstede, G. (1980). Culture's Consequences: International Differences in Work-Related Values. Beverly Hills, CA: Sage Publications.

Hofstede, G. (1980). Motivation, leadership, and organization: Do American theories apply abroad? Organizational Dynamics, 42-102.

Hofstede, G. (1983). The cultural relativity of organizational practices and theories. Journal of International Business Studies, 14(2), 75-89.

Hofstede, G., Neuijen, B., Ohayv, D.D., \& Sanders, G. (1990). Measuring organizational cultures: A qualitative and quantitative study across twenty cases. Administrative Science Quarterly, 35, 286-316.

Hofstede, G. (1994). Cultures and organizations - intercultural cooperation and its importance for survival. London: Harper Collins Business.

Hofstede, G. (1994). VSM 94 - Values Survey Module 1994 Manual. Geert Hofstede BV. Retrieved on April 4, 2011 from: http://www.geert-hofstede.com.

Hofstede, G. \& Bond, M.H. (1988). The Confucius connection: From cultural roots to economic growth. Organizational Dynamics, 16(4), 4-21.

Hofstede, G. (2001). Cultures Consequences: Comparing values, behaviors, institutions, and organizations across nations (2nd ed.). London: Sage Publications.

Hofstede, G., \& McCrae, R.R. (2004). Personality and culture revisited: Linking traits and dimensions of culture. Cross-Cultural Research, 38(1), 52-88.

Hofstede, G. \& Hofstede, G.J. (2005) Cultures and Organizations. Software of the Mind (2nd ed). New York, NY: McGraw-Hill.

Homans, C. (1958). Social behavior as exchange. American Journal of Sociology, 63, 597-606.

Hsu, M.K., Jiang, J.J., Klein, G., \& Tang, Z. (2002). Perceived career incentives and intent to leave. Information \& Management, 40, 361-369.

Hunt, D.M., \& Michael, C. (1983). Mentorship: a career training and development tool. Academy of Management Review, 8(3), 475-485.

Ibarra, H. (1995). Race, opportunity, and diversity of social circles in managerial networks. Academy of Management Journal, 38, 673-703. 
Iverson, R. D., \& Roy, P. (1994). A causal model of behavioral commitment: Evidence from a study of Australian blue-collar employees. Journal of Management, 20, 15-41.

Johnson, M. (1980). Mentors - the key to development and growth. Training and Development Journal, 34(7), 55, 57.

Johnson, B. \& Stevens, J.J. (2001). Confirmatory factor analysis of the school level environment questionnaire (SLEQ). International Journal of Learning Environments Research, 4(3), 325-344.

Johnson, W.B. (2007). Transformational supervision: When supervisors mentor. Professional Psychology: Research and Practice, 38, 259-267.

Jones, C. \& Jowett, V. (1997) Managing Facilities. Oxford: ButterworthHeinemann.

Kagan, T. (2000). History of Education. Selected Moments of the 20 ${ }^{\text {th }}$ Century. 1961: Theodore W. Schultz publishes Investment in Human Capital. Retrieved on September 6, 2003 from http://fcis.oise.utoronto.ca/ daniel_schugurensky/assignment1/1961schultz.html

Kaltenbach, H.M. (2012). A Concise Guide to Statistics. New York, NY: Springer. Page 68.

Kamenou, N. (2007, November). Methodological considerations in conducting research across gender, 'race', ethnicity and culture: a challenge to context specificity in diversity research methods. International Journal of Human Resource Management, 18(11), 1995-2010.

Kammeyer-Mueller, J.D., \& Judge, T.A. (2008). A quantitative review of mentoring research: test of a model. Journal of Vocational Behavior, 72, 269-283.

Kanter, R. (1977). Men and Women of the Corporation. New York, NY: Basic Books.

Kelly, M. (2008, May). Comparison of human resource management practices and perceptions of agri-business employees across three Indonesian subcultures. A Dissertation. College Station, TX, US.

Knowdell,R.L. (1996). Building a Career Development Program. Nine Steps for Effective Implementation. California: Davies-Black Publishing.

Kram, K. (1985). Mentoring at Work. Glenview: Scott, Foreman.

Kram, K. (1988). Mentoring at Work: Developmental Relationships in Organizational Life. Lanham, MD, US: University Press of America, Inc.

Kram, K., \& Hall, D. T. (1989). Mentoring as an antidoe to stress during corporate trauma. Human Resource Management, 28, 493-510. 
Kram, K., \& Hall, D. T. (1996). Mentoring in a context of diversity and turbulence. In E. Kossek, \& S. A. Lobel, eds., Managing diversity: Human resource strategy for transforming the workplace (pp. 108-136). Cambridge, MA: Blackwell.

Lam, T., Baum, T., \& Zhang, H. (2001). An investigation of employees' job satisfaction: the case of hotels in Hong Kong. Tourism Management, 22, 157-165.

Lam, T., Baum, T., \& Pine R. (2003). Subjective norms, effects on job satisfactions. Annals of Tourism Research. 30(1), 160-177.

Lankau, M. J., \& Scandura, T.A. (2002). An investigation of personal learning in mentoring relationships: Content, antecedents, and consequences. Academy of Management Journal, 45(4), 779-790.

Lankau, M., \& Scandura, T.A. (2007). Mentoring as a forum for personal learning in organizations. In B. Ragins, \& K. E. Kram, eds., The Handbook of Mentoring at Work: Theory, Research, and Practice (pp. 95-122). Los Angeles, CA: Sage Publications.

Lee, F.K., Dougherty, T.W., \& Turban, D.B. (2000). The role of personality and work values in mentoring programs. Review of Business, 21, 33-37.

Lee-Kelley, L., Blackman, D.A., \& Hurst, J.P. (2007). An exploratipon of the relationship between learning organizations and the retention of knowledge workers. The Learning Organization, 14(3), 204-221.

Lentz, E., \& Allen, T.D. (2005, April). The link between mentoring and the career plateau: Addressing the empirical gap. Paper presented at the 2005 Annual Conference of the Society for Industrial and Organizational Psychology, Los Angeles, CA.

Locurcio, R.V., \& Mitvalsky, K. (2002, April). Mentoring: A magnet for young engineers. (33, Ed.) Leadership and Management in Engineering, 31.

Lok, P., \& Crawford, J. (2003). The effect of organisational culture and leadership style on job satisfaction and organisational commitment: A cross-national comparison. Journal of Management Development, 23(4), 321-338.

Marsden, P.V., Cook, C.R., \& Kalleberg, A.L. (1993). Gender differences in organizational commitment: Influences of work positions and family roles. Work and Occupations, 20(3), 368-390.

Mathieu, J., \& Zajac, D.M. (1990). A review and meta-analysis of the antecedents, correlates, and consequences of organizational commitment. Psychological bulletin, 108, 171-194.

Mattei, N. (2001, October). Mentoring. Leadership and Management in Engineering, 41-48.

Matuszek, T., Self, D.R., \& Schraeder, M. (2008). Mentoring in an increasingly global workplace: facing the realities and challenges. Development and Learning in Organizations, 22(6), 18-20. 
McCrae, R. (2000). Trait psychology and the revival of personality and culture studies. American Behavioral Scientist, 44, 10-31.

McFarlin, D.B., \& Sweeney, P.D. (1992). Distributive and procedural justice as predictors of satisfaction with personal and organizational outcomes. Academy of Management Journal, 35, 626-637.

McGuire, D., Garavan, T.N., Murphy, J., O'Donnel, D., \& Saha, S.K.(2002). The cultural boundedness of theory and practice in HRD? Cross Cultural Management, 9(2), 25-44.

McKeen, C., \& Bujaki, M. (2007). Gender and mentoring. In B. Ragins, \& K. E. Kram, eds., The Handbook of Mentoring at Work: Theory, Research, and Practice (pp. 197-222). Los Angeles, CA: Sage Publications.

McKimm, J., Jollie, C., \& Hatter, M. (2007). Mentoring: Theory and practice. NHSE/Imperial College, School of Medicine.

McLean, G.N., \& McLean, L. (2001). If we can't define HRD in one country, how can we define it in an international context? Human Resource Development International, 4(3), 313-326.

Megginson, D. \& Clutterbuck, D. (1995) Mentoring in Action: A practical guide for managers. London: Kogan Page.

Mentoring Program Handbook. (2003, May). Retrieved June 10, 2010, from NASA: nasapeople.nasa.gov/training/coachmentor/mentorguide.pdf

Meyer, J.P., Allen, N.J., \& Gellatly, I.R. (1990). Affective and continuance commitment to the organization: Evaluation of measures and analysis of concurrent and time-lagged relations. Journal of Applied Psychology, 75, 710-720.

Meyer, J.P., \& Allen, N.J. (1997). Commitment in the workplace: Theory, research and application. Thousand Oaks: CA.

Meyer, J.P., Stanley, D.J., Herscovitch, L., \& Topolnytsky, L. (2002). Affective, continuance and normative commitment to the organization: A meta-analysis of antecendents, correlates, and consequences. Journal of Vocational Behavior, 61, 20-52.

Mezias, J., \& Scandura, T.A. (2005). A needs-driven approach to expatriate adjustment and career development: A multiple mentoring perspective. Journal of International Business Studies, 36, 519-538.

Miller, R.G. (1986). Beyond ANOVA, Basics of Applied Statistics. New York, NY: John Wiley \& Sons Inc.

Miroshnik, V. (2002). Culture and international management: a review. Journal of Management Development, 21(7), 521-544. 
Morden, T. (1995). International culture and management. Management Decision, 33(2), 16-21.

Mowday, R.T., Steers, R.M., \& Porter, L.W. (1979). The measurement of organizational commitment. Journal of Vocational Behavior, 14, 224-247.

Mullen, E. (1994). Framing the mentoring relationship as an information exchange. Human Resource Management Review, 4, 257-281.

Mullen, E., \& Noe, R.A. (1999). The mentoring information exchange: When do mentors seek information from their proteges? Journal of Organizational Behavior, 20, 233-242.

Murray, M. (1991). Beyond the myths and magic of mentoring. San Francisco, CA: Jossey-Bass.

Murrell, A., \& James, E.H. (2001). Gender and diversity within organizations. Sex Roles, 45, 243-257.

Newman, K., \& Nollen, S. (1996). Culture and congruence: The fit between management practices and nations. Journal of International Business Studies, 27(4), 753-779.

Noe, R. (1988). An investigation of the determinants of successful assigned mentoring relationships. Personnel Psychology, 41, 457-479.

Noe, R. A., Greenberger, D. B., \& Wang, S. (2002). Mentoring: What we know and where we might go. Research in Personnel and Human Resources Management, 21, 129-173.

Olian, J.D., Carroll, S.J., Giannantonio, C.M., \& Feren, D.B. (1988). What do proteges look for in a mentor? Results of three experimental studies. Journal of Vocational Behavior, $33(1), 15-37$.

Oyserman, D., Coon, H.M., \& Kemmelmeier, M. (2002). Rethinking individualsim and collectivism: Evaluation of theoretical assumptions and meta-analyses. Psycological Bulletin, 128(1), 3-72.

Ozkalp, E., Kirel, C., Sungur, Z., \& Ozdemir, A.A. (2008, August). Mentoring relations in the aircraft industry: a case study in Turkey. International Journal of Evidence Based Coaching and Mentoring, 6(2), 67-77.

Pallant, J. (2005). SPSS Survival Manual ( $2^{\text {nd }}$ ed.) United Kingdom. Open University Press.

Parise, M.R., \& Forret, M.L. (2008). Formal mentoring programs: The relationship of program design and support to mentors' perceptions of benefits and costs. Journal of Vocational Behavior, 72, 225-240.

Pearson, C. (1991). An assessment of extrinsic feedback on participation, role, perceptions, motivation, and job satisfaction in a self-managed system for monitoring group achievement. Human Relations, 44(5), 517-537. 
Porter, L.W. \& Steers, R.M. (1973). Organizational, work, and personal factors in employee turnover and absenteeism. Psychological Bulletin, 80, 151-176.

Porter, L.W., Boulian, P.V., Mowday, R.T., \& Steers, R.M. (1974) Organizational commitment, job satisfaction, and turnover among psychiatric technicians. Journal of Applied Psychology, 59, 603-609.

Raabe, B., \& Beehr, T.A. (2003). Formal mentoring versus supervisor and coworker relationships: differences in perceptions and impact. Journal of Organizational Behavior, 24, 271-293.

Ragins, B.R (1989). Barriers to mentoring: The female manager's dilemma. Human Relations, 42(1), 1-22.

Ragins, B.R (1997). Diversified mentoring relationships in organizations: A power perspective. The Academy of Management Journal, 22(2), 482-521.

Ragins, B.R., \& Scandura, T.A. (1999). Burden or blessing? Expected costs and benefits of being a mentor. Journal of Organizational Behavior, 20, 493-509.

Ragins, B.R, \& Cotton, J.L. (1999). Mentor functions and outcomes: A comparison of men and women in formal and informal mentoring relationships. Journal of Applied Psychology, $84,529-550$.

Ragins, B.R., Cotton, J.L., \& Miller, J.S. (2000, December). Marginal mentoring: The effects of type of mentor, quality of relationship, and program design on work and career attitudes. The Academy of Management Journal, 43(6), 1177-1194.

Ragins, B.R., \& Kram, K.A. (2007). The roots and meaning of mentoring, In B. R. Ragins \& K. E. Kram, eds., The handbook of mentoring at work: Theory, research, and practice (pp. 3-20). Thousand Oaks, CA: Sage Publications.

Ramaswami, A., \& Dreher, G.F. (2007). The benefits associated with workplace mentoring relationships. In T. A. Allen and L. Eby. eds., Handbook of mentoring: A multiple perspectives approach (Ch. 13). Blackwell Publishing.

Read, T.R.C \& Cressie, N.A.C. (1988). Goodness-of-Fit statistics for discrete multivariate data. New York: Springer-Verilag.

Rosser, M. (2004, December). Chief Executive Officers: Their mentoring relationships. A Dissertation. College Station, TX, US.

Rowden. (2002). The relationship between workplace learning and jobsatisfaction in U.S. small to midsize businesses. Human Resource Development Quarterly, 13(4), 407-425.

Rowden, R.W., \& Conine, Jr., C.T. (2005). The impact of workplace learning on job satisfaction in small US commercial banks. Journal of Workplace Learning, 17(4), 215-230. 
Russell, J. (2006, January). Mentoring in Engineering. Leadership and Management in Engineering, 34-37.

Russell J.E.A. \& Adams D.M. (1997). The changing nature of mentoring in organizations: An introduction to the special issue on mentoring in organizations. Journal of Vocational Behavior, 51(1), 1-14.

Russell, J.S., \& Nelson, J. (2009, January). Completing the circle of professional development through leadership and mentoring. Leadership and Management in Engineering, 40-42.

Ruxton, G.C. (July/August 2006). The unequal variance $t$-test is an underused alternative to Student's $t$-test and the Mann-Whitney $U$ test. Behavioral Ecology, 17 (4): 688-690.

Sadler, P. J., \& Hofstede, G. (1972). Leadership styles: Preferences and perceptions of employees of an international company in different countries. Leadership Styles, 26, $43-$ 63.

Samad, S. (2005). Unraveling the organizational commitment and job performance relationship: Exploring the moderating effect of job satisfaction. The Business Review, 4(2), 79-84.

Samad, S. (2006). Predicting turnover intentions: The case of Malaysian government doctors. Journal of American Academy of Business, 8(2), 113-120.

Satterwaite, F.E. (1946). An approximate distribution of estimates of variance components. Biometrics Bulletin, 2:110-4.

Scandura, T. A. (1992). Mentorship and career mobility: An empirical investigation. Journal of Organizational Behavior, 13, 169-174.

Scandura, T. A., \& Schriesheim, C. A. (1994). Leader-member exchange and supervisor career mentoring as complementary constructs in leadership research. Academy of Management Journal, 37(6), 1588-1602.

Scandura, T.A. \& Siegel, P.H. (1995). Mentoring as organizational learning during a corporate Merger paper presented at the 1995 National Academy of Management meetings, Vancouver, Canada.

Scandura, T.A., \& Williams, E.A. (2001). An investigation of the moderating effects of gender on the relationships between mentorship initiation and protégé perceptions of mentoring functions. Journal of Vocational Behavior, 59, 342-363.

Schmidt, S. (2007). The relationship between satisfaction with workplace training and overall job satisfaction. Human Resource Development Quarterly, 18(4), 481-498.

Seibert, S. (1999). The effectiveness of facilitated mantoring: A longitudinal quasi-experiment. Journal of Vocational Behavior, 54, 483-502. 
Shea, G.F. (1994). Mentoring: Helping employees reach their full potential. New York: American Management Association.

Schultz, T. W. (1961). Investment in Human Capital. The American Economic Review $(51: 1)$.

Sheldon, M.E. (1971). Investment and involvement as mechanism producing organizational commitment, Administrative Science Quarterly, 16:143-150.

Simmons, T. \& Hinkin, T. (2001). The Effect of Employee Turnover on Hotel Profits. A Test Across Multiple Hotels. Cornell Hotel and Restaurant Administration Quarterly, August, 65-69.

Smith, B., Bond, M., \& Kagitcjbas, C. (2006). Understanding social psychology across cultures: Living and working in a changing world. London: Sage Publications.

Smith, H. (1936). The problem of comparing the results of two experiments with unequal errors. Journal of Scientific and Industrial Research, 9:211-2.

Sparrow, P., Jackson, S., \& Schuler, R.(1994). Convergence or divergence? Human resource practices and policies for competitive advantage world-wide. International Journal of Human Resource Management, 267-299.

Tabachnick, B.G. \& Fidell, L.S. (2001). Using multivariate statistics $\left(4^{\text {th }}\right.$ ed.). New York, NY: Harper Cllins. Chapter 14.

Tayeb, M. (1995). The competitive advantage of nations: the role of HRM and its socio-cultural context. The International Journal of Human Resource Management, 6(3), 588-605.

Taylor, E. (1924). Primitive Culture. Gloucester, MA: Smith.

Thibaut, J.W., \& Kelley, H.H. (1959). The social psychology of groups. New York: Wiley.

Thomas, D. (1990). The impact of race on managers' experiences of developmental relationships (mentoring and sponsorship): An intra-organizational study. Journal of Organizational Behavior, 11, 479-492.

Thomas, D. (1993). Racial dynamics in cross-race developmental relationships. Administrative Science Quarterly, 11, 479-492.

Thomas, D., \& Alderfer, C. (1989). The influence of race on career dynamics theory and reserach on minority career experiences. In M. Arthur, D. T. Hall, \& B. S. Lawrence, eds., Handbook of Career Theory (pp. 133-158). New York, NY: Cambridge University Press.

Tolson, H. (Spring 2010). EDAD/EHRD - 690A - STAT 1. Texas A\&M University Course Packet. 
Trolier, T.K. (1986). Stereotypes and stereotyping: An overview of the cognitive approach. In J. Dovidio \& S. Gaertner, eds., Prejudice, discrimination, and racism (pp. 127-163). Orlando, FL: Academic Press.

Trompenaars, F., \& Hampden-Turner, C. (1998). Riding the Waves of Culture. New York, NY: McGraw Hill.

Turban, D.B., \& Dougherty, T.W. (1994). Role of protege personality in receipt of mentoring and career success. Academy of Management Journal, 37, 688-702.

Turban, D.B., Dougherty, T.W., \& Lee, F.K., (2002). Gender, race, and perceived similarity effects in developmental relationships: The moderating role of relationship duration. Journal of Vocational Behavior, (61), 240-262.

Turban, D.B., \& Jones, A.P. (1988). Supervisor-subordinate similarity: Types, effects and mechanisms. Journal of Applied Psychology, 73, 228-234.

Turban, D.B., \& Lee, F.K. (2007). The role of personality in mentoring relationships. In B. Ragins, \& K.E. Kram, The Handbook of Mentoring at Work: Theory, Research, and Practice (pp. 21-50). Los Angeles, CA: Sage Publications.

Turner, J. (1982). Towards a cognitive redefinition of the social group. In H. Tajfel, Social identity and intergroup relations, In S. Worchel and L. W. Austin, eds, Psychology of Intergroup Relations. Chigago: Nelson-Hall. Cambridge: Cambridge University Press.

Tyler, K. (1998). Mentoring programs link employees and experienced execs. HR Magazine, 43(5), 98-103.

Ullman. J.B. (1996). Structural equation modeling (In: Using Multivariate Statistics, Third Edition, B.G. Tabachnick and L.S. Fidell, eds., New York, NY: HarperCollins College Publishers.

Voelz, V.A. (2006). Principle Component Analysis and other data reduction techniques. Math Bio Boot Camp - 2006. As retrieved August 4, 2011 from: http://www.stanford.edu/ vvoelz/lectures/PCA.pdf.

Vroom, V. (1964). Work and Motivation. San Francisco, CA: Jossey-Bass.

Wanberg, C.R., Kammeyer-Mueller, J., \& Marchese, M. (2006). Mentor and protege predictors and outcomes of mentoring in a formal mentoring program. Journal of Vocational Behavior, 69, 410-423.

Wanberg, C., Welsh, E. T., \& Hezlett, S. A. (2003). Mentoring research: A review and dynamic process model. Research in Personnel and Human Resource Management, 22, 39-124.

Wang, J., Tolson, H., Chiang, T., \& Huang, T. (2010, April). An exploratory factor analysis of workplace learning, job satisfaction, and organizational commitment in small to midsize enterprises in Taiwan. Human Resource Development International, 13(2), 147-163. 
Ward, E.A. \& Davis, E. (1995). The effect of benefit satisfaction on organizational commitment. Compensation and Benefits Management (11), 35-40.

Wayne, S.J., Shore, L.M., \& Liden, R.C. (1993). Perceived organizational support and leadermember exchange: A social exchange perspective. Academy of Management Journal, 40, 82-111.

Weiss, D.J., Dawis, R.V., England, G.W., \& Lofquist, L.H. (1967). Manual for the Minnesota satisfaction questionnaire. Minneapolis, MN: University of Minnesota.

Welch, B.L. (1938). The significance of the difference between two means when the population variances are unequal. Biometrika, 29:350-62.

Welch, B.L. (1947). The generalisation of students problem when several different population variances are involved. Biometrika, 34:23-35.

Wever, K. (1995, September). Human resource management and organizational strategies in German- and US-owned companies. The International Journal of Human Resource Management, 6(3), 606-625.

Wilson, J.A., \& Elman, N.S. (1990). Organizational benefits of mentoring. Academy of Management Executive, 4, 88-94.

Wright, B.E., \& Davis, B.S. (2003). Job Satisfaction in the Public Sector: The Role of the Work Environment. American Review of Public Administration, 33 (1), 70-90.

Wright, P., McMahan, G. C., \& McWilliams, A. (1994). Human resources as a source of competitive advantage: A resource-based perspective. International Journal of Human Resource Management, 5, 299-326.

Yang, B., Wang, Y., \& Drewry, A.W. (2009). Does it matter where to conduct training? Accounting for cultural factors. Human Resource Management Review, 19, 324-333.

Zar, J.H. (1999). Biostatistical Analysis, fourth edition. New Jersey: Prentice Hall.

Zey, M. (1984). The mentor connection. Homewood, IL: Dow Jones - Irwin.

Zey, M. (1985). Mentor programs: Making the right moves. Personnel Journal, 64, 53-57. 


\section{APPENDIX 1}

\section{Information Sheet}

You have been asked to participate in a research study where the researcher will investigate cultural factors that may affect job satisfaction, organizational commitment, and participation in formal mentoring programs. You were selected to be a possible participant because you are an employee within Baker Hughes, Intl. A total of 5700 people have been asked to participate in this study. The purpose of this study is to investigate cultural differences and values that may affect job satisfaction, organizational commitment, and participation in formal mentoring programs.

If you agree to participate in this study, you will be asked to fill out and return a survey questionnaire that will be sent to you via email. This survey will be available to you for three weeks between March 21, 2011 and April 15, 2011. The survey will take between15-20 minutes to complete, from start to finish, if there are no interruptions. The risks associated with this study are discomfort due to the time taken to fill the survey out. The benefits due to the study are that your responses might help the corporation decide to implement a formal mentoring program in order to enhance your job satisfaction within the organization. You understand that you will receive no monetary or other benefits.

This study is confidential. Your email address will not be visible to other participants doing the survey. Your name or any information that identifies you will be kept confidential by the researcher. The records of this study will be kept private and any information linking you to your information will be destroyed by the researcher as soon as the information is recorded. No identifiers linking you to the study will be included in any sort of report that may be published. Your decision whether or not to participate will not affect my current or future relations with Texas A\&M University. If you decide to participate, you will be free to refuse to answer any of the questions that may make you uncomfortable. You can withdraw at any time without your relations with Baker Hughes, the job, management, benefits, etc., being affected. You can contact Hanna Hayes, the researcher, by email at hannahayes@neo.tamu.edu, or by telephone at 828-280-4783, with any questions about this study. You can also contact the researcher's advisor, Dr. Larry Dooley, at 1-dooley@tamu.edu.

This research study has been reviewed by the Institutional Review Board - Human Subjects in Research, Texas A\&M University. For research related problems or questions regarding subjects' rights, you can contact the Institutional Review Board through Ms. Angelia M. Raines, Director of Research Compliance, Office of the Vice President of Research at 979-458-4067, araines@,vprmail.tamu.edu

Please be sure you have read the above information, asked questions and received answers to your satisfaction. You are encouraged to keep a copy of the information sheet for your records. 


\title{
APPENDIX 2
}

\author{
Employee Characteristics Survey
}

\section{Introduction:}

The following questionnaire is an attempt to get an overall understanding of your values and characteristics; moreover, this questionnaire is used to measure your interest in a formal mentoring program. The big question often asked by organizations is whether training and development is helping individuals succeed in their jobs by adding to their occupational knowledge in the workplace? Through your responses, we hope to understand this critical mentoring question often asked in business and industry.

\section{Instructions on completing the questions:}

The instrument has 44 items and consists of five pages. There are five sections. Each section contains a scale from strongly disagree to strongly agree, or a scale of utmost importance to very little or no importance. Please indicate the extent to which you agree or disagree with the statement by marking any one number on the scale. Please respond to all items such that the researcher can have complete information in order to help improve future training efforts. Please be as candid as possible with your answers because the information you provide will help to improve future training efforts that you or your co-workers may attend.

Statement of Confidentiality: The researcher will not reveal your identity to any person or organization.

If you have any questions please feel free to email at hannahayes@neo.tamu.edu. Thank you!

\section{SECTION ONE - MENTORING}

Mentoring, defined by Bowen (1985), "Mentoring occurs when a senior person (the mentor) in terms of age and experience undertakes to provide information, advice, and emotional support to a junior person (the protégé) in a relationship which is set formally by the constraints of the program and lasts for a limited period of time" (p.31).

$7=$ Strongly Agree

$6=$ Moderately Agree

$5=$ Slightly Agree

$4=$ Neither Disagree or Agree

$3=$ Slightly Disagree

$2=$ Moderately Disagree

$1=$ Strongly Disagree

1. I have someone at work who takes a personal interest in my career. 
2. I have someone at work who helps me coordinate professional goals.

3. I have someone at work who devotes special time and consideration to my career.

4. I share my personal problems with someone at work.

5. I exchange confidences with someone in a senior position at work.

6. I consider someone in a senior position to be a friend.

As you may know, the Baker Hughes LEAD Program provides information, encouragement, and support through its mentorships. Each mentee is matched with an experienced mentor (Training Engineer) who provides guidance and support to the mentee through mentoring relationship. Please answer the following questions as clearly as possible.

Are you currently participating in a mentoring relationship through the Baker Hughes LEAD Program (i.e. have you been placed with a Training Engineer)?

Yes $\square$ No $\square$

If you are currently participating in a mentoring relationship (placed with a Training Engineer) though the Baker Hughes LEAD Program, is this relationship of benefit to you?

Yes $\square$ No $\square$

If you are not currently participating in the Baker Hughes LEAD Program mentoring relationship (placed with a Training Engineer), would you like to be assigned to a mentor (Training Engineer)?

Yes $\square$ No $\square$

Do you have concerns about being mentored (placed with a Training Engineer) through the Baker Hughes LEAD Program?

Yes $\square$ No $\square$

\section{SECTION TWO}

$7=$ Strongly Agree

$6=$ Moderately Agree

$5=$ Slightly Agree

$4=$ Neither Disagree or Agree

$3=$ Slightly Disagree

$2=$ Moderately Disagree

$1=$ Strongly Disagree

All in all, I am satisfied with my job.

In general, I don't like my job.

In general, I like working here. 


\section{SECTION THREE}

$4=$ Strongly Agree

$3=$ Moderately Agree

$2=$ Moderately Disagree

$1=$ Strongly Disagree

10. I am willing to work harder than I have to in order to help this organization succeed.

11. I feel very little loyalty to this organization

12. I would take almost any job to keep working for this organization.

13. I find that my values and the agency's values are very similar.

14. I am proud to be working for this organization.

15. I would turn down another job for more pay in order to stay with this organization.

\section{SECTION FOUR - VALUES}

$1=$ of utmost importance

$2=$ very important

$3=$ of moderate importance

$4=$ of little importance

$5=$ of very little or no importance

Please think of an ideal job. In choosing an ideal job, how important would it be to you to:

16. have sufficient time for my personal or family life.

17. have good physical working conditions (good ventilation and lighting, adequate work space, etc.).

18. have a good working relationship with my direct superior.

19. have security of employment.

20. work with people who cooperate well with one another.

21. be consulted by my direct superior in his/her decisions.

22. have an opportunity for advancement to higher level jobs.

23. have an element of variety and adventure in the job.

In your private life, how important is each of the following to you? (please circle one answer in each line across):

24. Personal steadiness and stability

25. Thrift

26. Persistence (perseverance)

27. Respect for tradition

28. How often do you feel nervous or tense at work?

1. never

2. seldom

3. sometimes 


$$
\begin{array}{ll}
\text { 4. } & \text { usually } \\
\text { 5. } & \text { always }
\end{array}
$$

29. How frequently, in your experience, are subordinates afraid to express disagreement with their superiors?

$$
\begin{array}{ll}
\text { 1. } & \text { very seldom } \\
2 . & \text { seldom } \\
3 . & \text { sometimes } \\
\text { 4. } & \text { frequently } \\
\text { 5. } & \text { very frequently }
\end{array}
$$

To what extent do you agree or disagree with each of the following statements?

$1=$ strongly agree

$2=$ agree

$3=$ undecided

$4=$ disagree

$5=$ strongly disagree

30. Most people can be trusted.

31. One can be a good manager without having precise answers to most questions that subordinates may raise about their work.

32. An organization structure in which certain subordinates have two bosses should be avoided at all costs.

33. Competition between employees usually does more harm than good.

34. A company's or organization's rules should not be broken - not even when the employee thinks it is in the company's best interest.

35. When people have failed in life it is often their own fault.

\section{SECTION FIVE}

Some information about yourself (for statistical purposes):

36. Are you:

1. male

2. female

37. How old are you?

1. Under 20

2. 20-24

3. $25-29$

4. 30-34

5. $\quad 35-39$

6. $40-49$

7. $\quad 50-59$

8. 60 or over 
38. How many years of formal school education (or the equivalent) did you complete (starting with primary school)?

$\begin{array}{ll}\text { 1. } & 10 \text { years or less } \\ 2 . & 11 \text { years } \\ 3 . & 12 \text { years } \\ 4 . & 13 \text { years } \\ 5 . & 14 \text { years } \\ 6 . & 15 \text { years } \\ 7 . & 16 \text { years } \\ 8 . & 17 \text { years } \\ 9 . & 18 \text { years or over }\end{array}$

39. What is the highest degree you have completed?

1. 12th grade, no diploma

2. High School Degree/Diploma or equivalent (GED)

3. Some college/university but no degree

4. Diploma or certificate from a vocational/technical, trade or business school

5. Associate degree in college - Occupational/vocational program

6. Associate degree in college - Academic program

7. Bachelor's degree (For example: BA, AB, BS)

8. Master's degree (For example: MA, MS, MEng, MEd, MSW, MBA)

9. Professional School Degree (For example: MD,DDS,DVM,LLB,JD)

10. Doctorate degree (For example: $\mathrm{PhD}, \mathrm{EdD}$ )

40. How long have you been employed with Baker Hughes?

1. Less than six (6) months

2. Six (6) months to less than One (1) year

3. One (1) year to less than Three (3) years

4. Three (3) years to less than Five (5) years

5. Five (5) years to less than Eight (8) years

6. Eight (8) years to less than Ten (10) years

7. More than Ten (10) years

41. How long have you been employed at your current position?

1. Less than six (6) months

2. Six (6) months to less than One (1) year

3. One (1) year to less than Three (3) years

4. Three (3) years to less than Five (5) years

5. Five (5) years to less than Eight (8) years

6 . Eight (8) years to less than Ten (10) years

7. More than Ten (10) years

42. What is your present country of citizenship?

43. What is your original country of citizenship?

44. In what country were you born?

Thank you very much for your cooperation! 


\section{APPENDIX 3}

Responses by Region

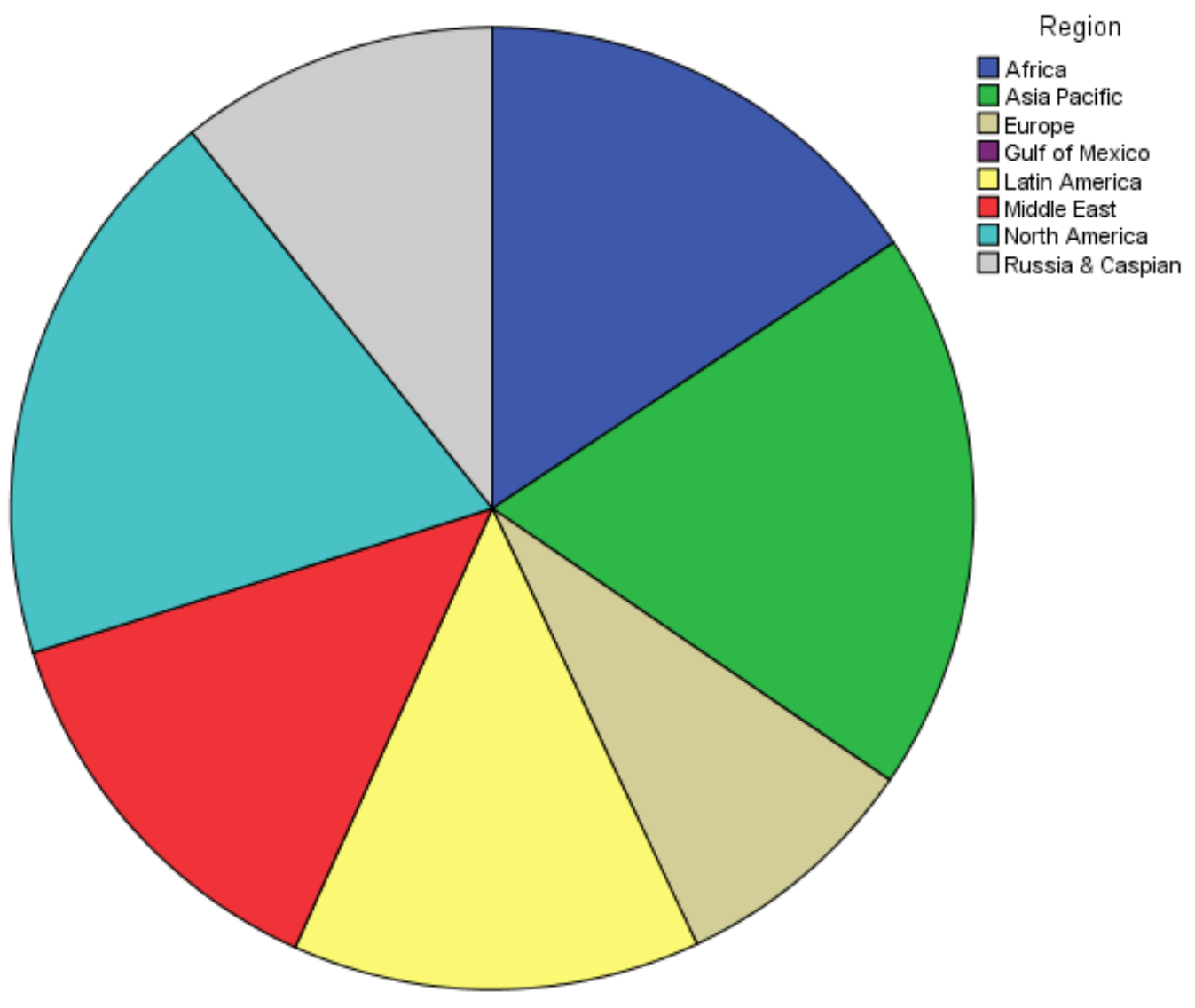




\section{APPENDIX 4}

\section{Responses by Country}

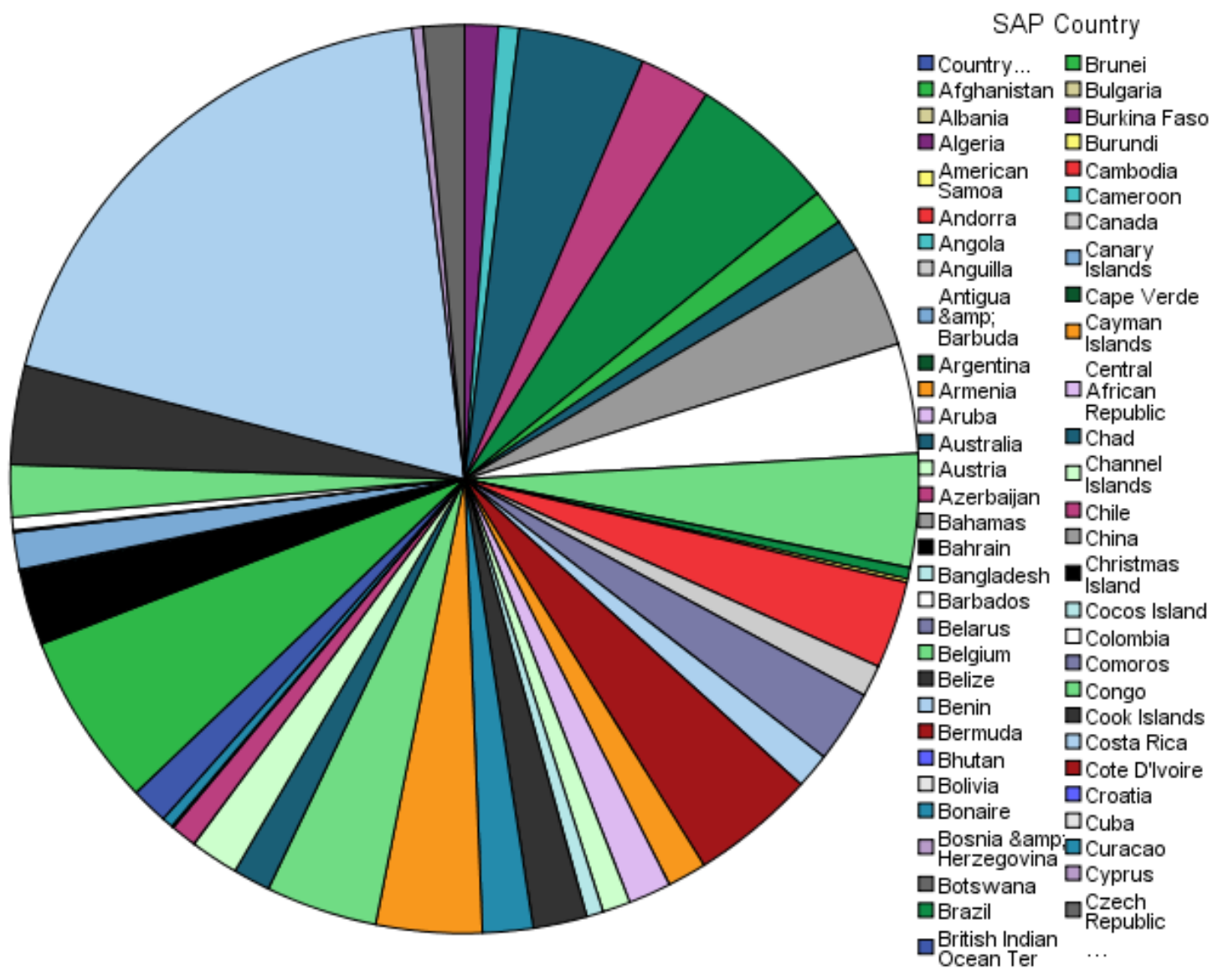




\section{APPENDIX 5}

Summary of ANOVA for Cultural Indices across Years Employed

\begin{tabular}{|l|c|c|c|c|c|}
\hline & IDV & PDI & MAS & LTO & UAI \\
\hline Entire sample & 0.071 & 0.980 & 0.041 & 0.902 & 0.027 \\
\hline $\begin{array}{l}\text { Eastern } \\
\text { Hemisphere }\end{array}$ & 0.037 & 0.754 & 0.038 & 0.419 & 0.095 \\
\hline $\begin{array}{l}\text { Western } \\
\text { Hemisphere }\end{array}$ & 0.950 & 0.261 & 0.203 & 0.329 & 0.054 \\
\hline Africa & 0.996 & 0.059 & 0.423 & 0.936 & 0.394 \\
\hline Asia Pacific & 0.301 & 0.681 & 0.177 & 0.334 & 0.334 \\
\hline Europe & 0.315 & 0.526 & 0.607 & 0.385 & 0.631 \\
\hline Latin America & 0.978 & 0.189 & 0.789 & 0.539 & 0.026 \\
\hline Middle East & 0.020 & 0.767 & 0.462 & 0.267 & 0.336 \\
\hline North America & 0.826 & 0.668 & 0.060 & 0.348 & 0.175 \\
\hline $\begin{array}{l}\text { Russia \& } \\
\text { Caspian }\end{array}$ & 0.616 & 0.798 & 0.465 & 0.951 & 0.489 \\
\hline
\end{tabular}

For individualism, there are significant differences for time employed when viewed from the entirety, the eastern hemisphere, and the Middle East. For power distance, the only significant difference across time employed is in the region of Africa. For masculinity, there are significant differences for time employed when viewed from the entirety, the eastern hemisphere, and the region of North America. For long term orientation, there are no differences across time employed. For uncertainty avoidance, there are significant differences for time employed when viewed from the entirety, the eastern and western hemisphere, and the region of Latin America. 


\section{APPENDIX 6}

Summary of ANOVA for Cultural Indices across Years Employed in Current Position

\begin{tabular}{|l|c|c|c|c|c|}
\hline & IDV & PDI & MAS & LTO & UAI \\
\hline Entire sample & 0.435 & 0.530 & 0.051 & 0.350 & 0.001 \\
\hline $\begin{array}{l}\text { Eastern } \\
\text { Hemisphere }\end{array}$ & 0.259 & 0.913 & 0.028 & 0.436 & 0.061 \\
\hline $\begin{array}{l}\text { Western } \\
\text { Hemisphere }\end{array}$ & 0.998 & 0.316 & 0.252 & 0.218 & 0.018 \\
\hline Africa & 0.611 & 0.593 & 0.824 & 0.984 & 0.781 \\
\hline Asia Pacific & 0.488 & 0.604 & 0.018 & 0.150 & 0.502 \\
\hline Europe & 0.010 & 0.490 & 0.532 & 0.011 & 0.513 \\
\hline Latin America & 0.682 & 0.534 & 0.545 & 0.832 & 0.013 \\
\hline $\begin{array}{l}\text { Middle East } \\
\text { North America }\end{array}$ & 0.451 & 0.665 & 0.294 & 0.586 & 0.266 \\
\hline $\begin{array}{l}\text { Russia \& } \\
\text { Caspian }\end{array}$ & 0.559 & 0.999 & 0.870 & 0.981 & 0.007 \\
\hline
\end{tabular}

For individualism, there is a significant difference for time employed in current position when viewed from Europe. For power distance, there are no differences across time employed in current position. For masculinity, there are significant differences for time employed in current position when viewed from the entirety, the eastern hemisphere, and the regions of Asia Pacific and North America. For long term orientation, there are significant differences for time employed in current position when viewed from Europe. For uncertainty avoidance, there are significant differences for time employed when viewed from the entirety, the eastern and western hemisphere, and the regions of Latin America and Russia \& Caspian. 


\section{APPENDIX 7}

Normality Plots of Variables Used

For a distribution to be normal, the points should fall on the line or be symmetric about the line
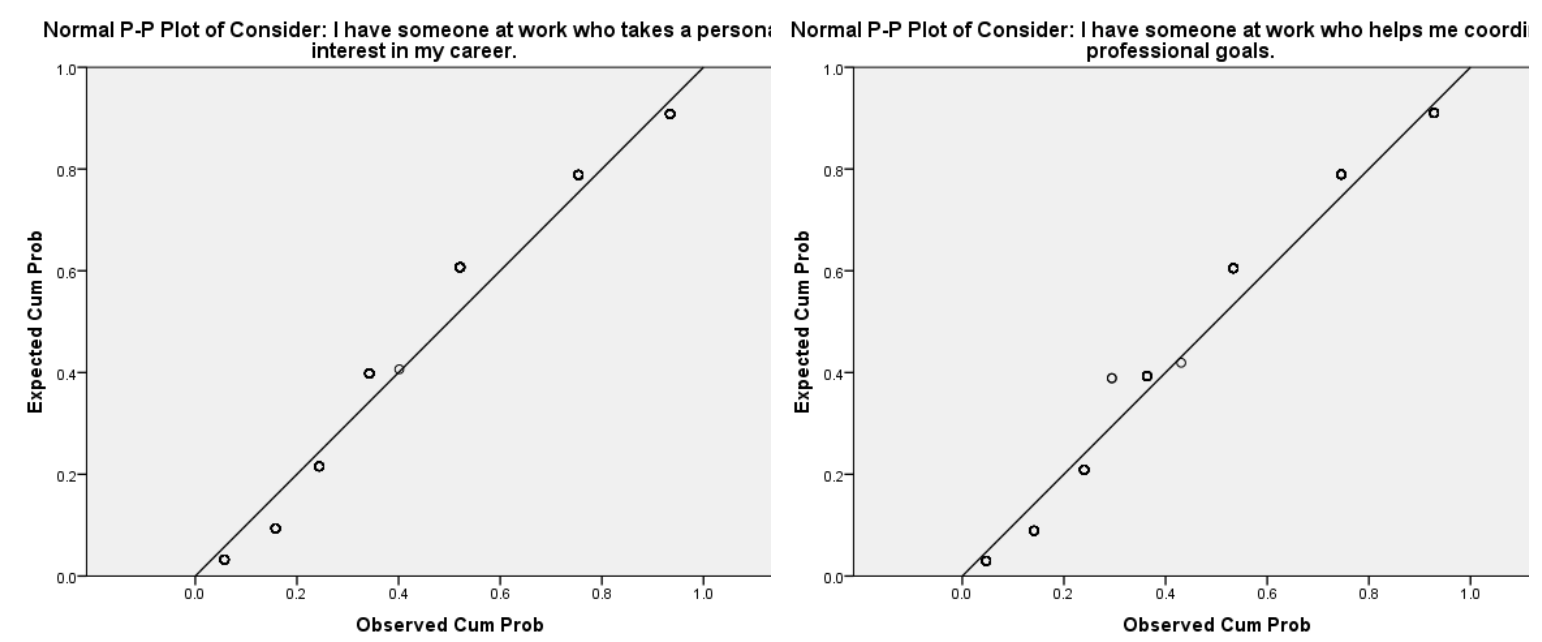

CS1

CS2

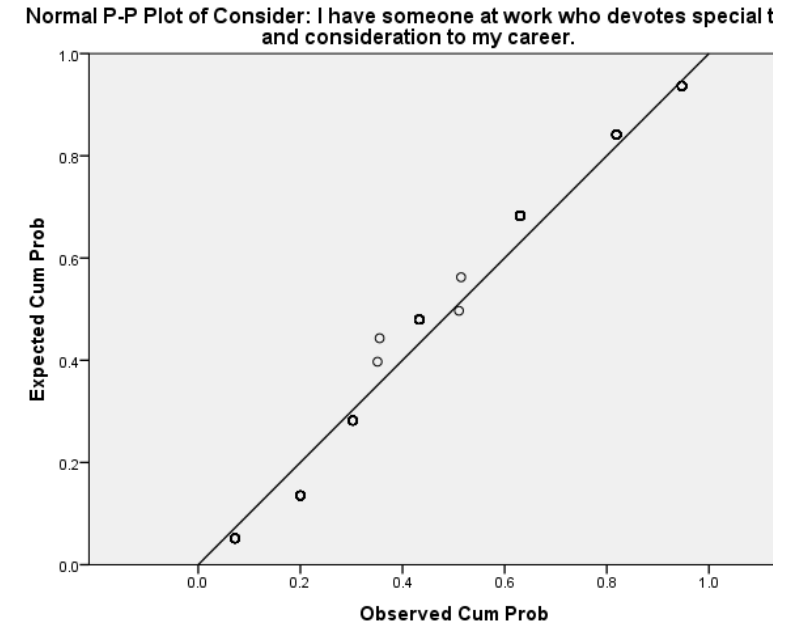

Normal P-P Plot of Consider: I share my personal problems with someon

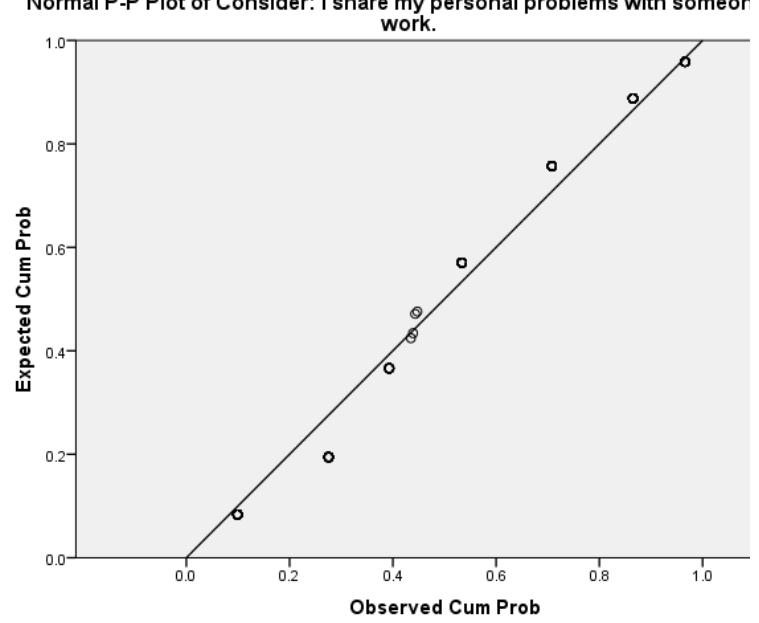

CS3

JS1 
Normal P-P Plot of Consider: I exchange confidences with someone in a senic

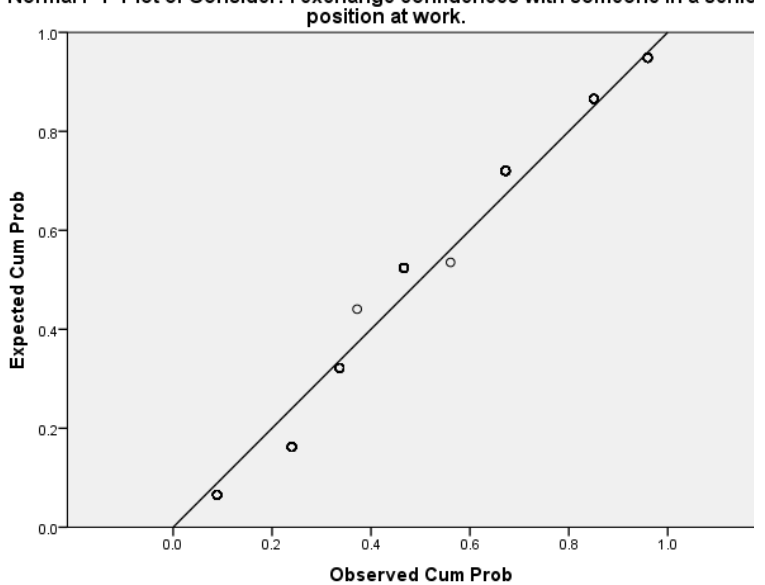

JS2

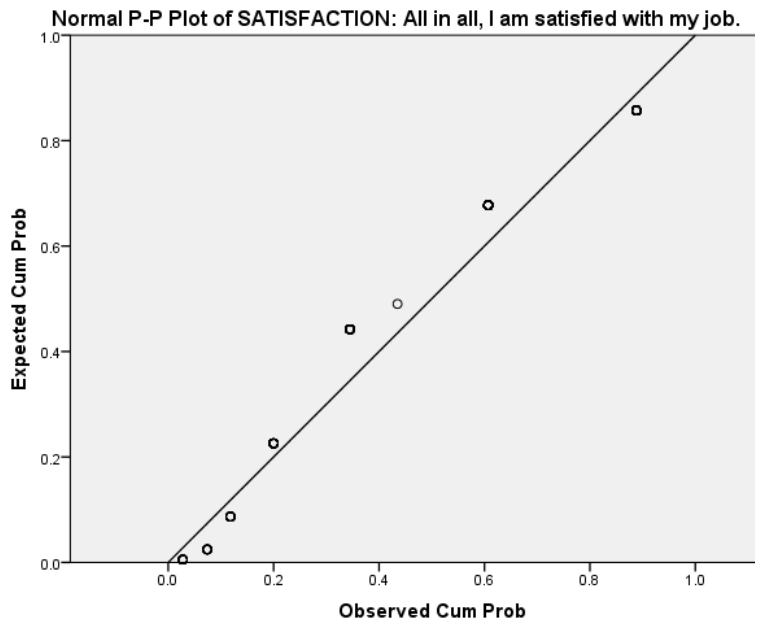

ES1

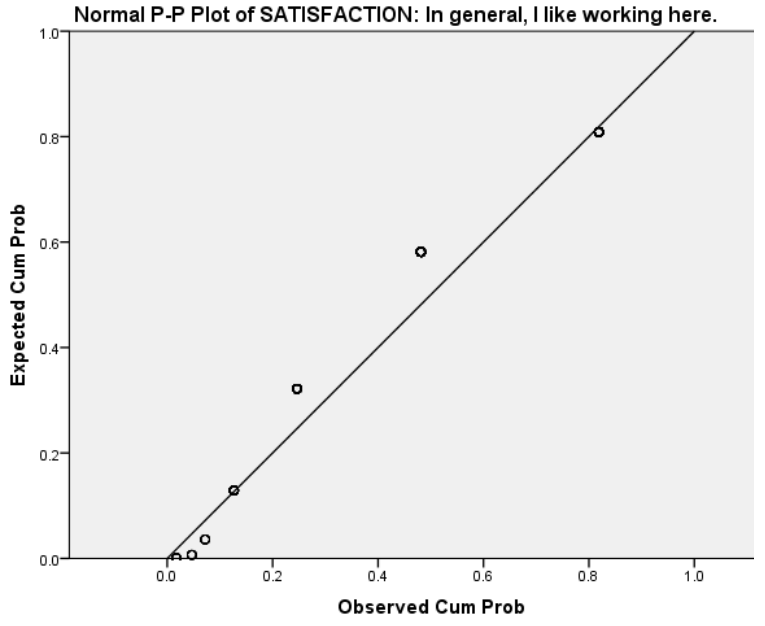

ES3
Normal P-P Plot of Consider: I consider someone in a senior position to be :

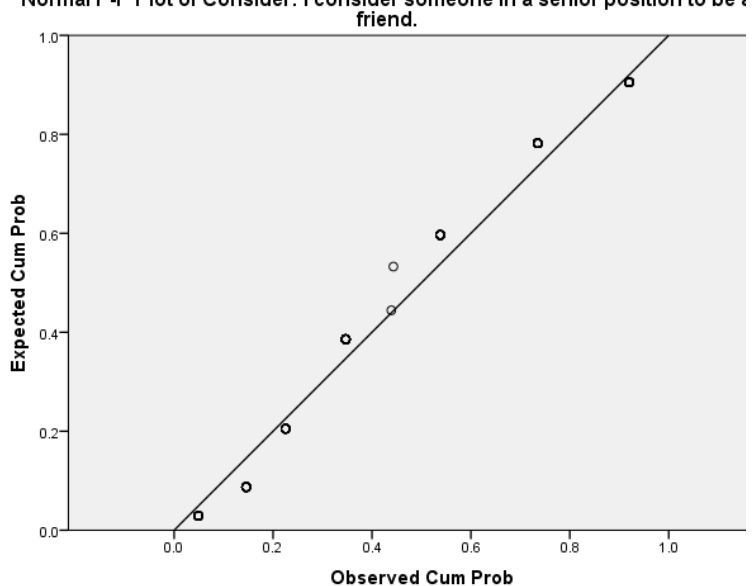

JS3

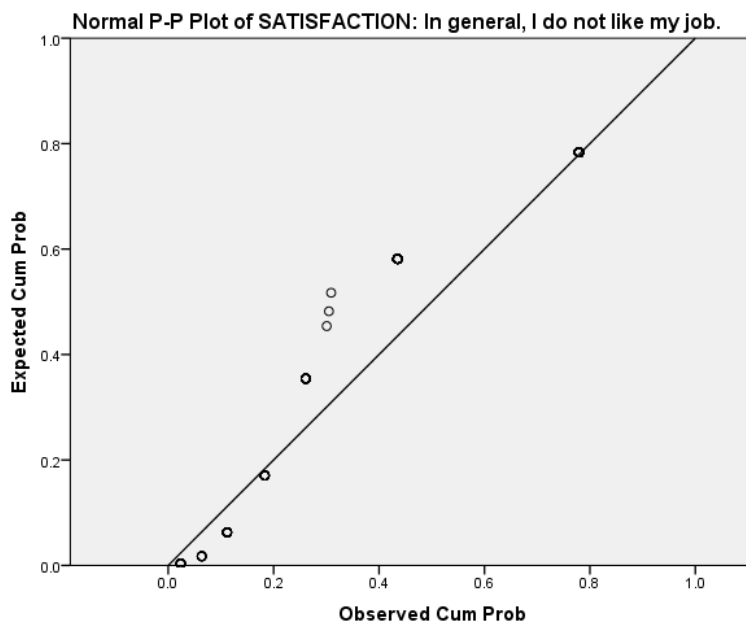

ES2

Normal P-P Plot of ENVIRONMENT: I am willing to work harder than I have

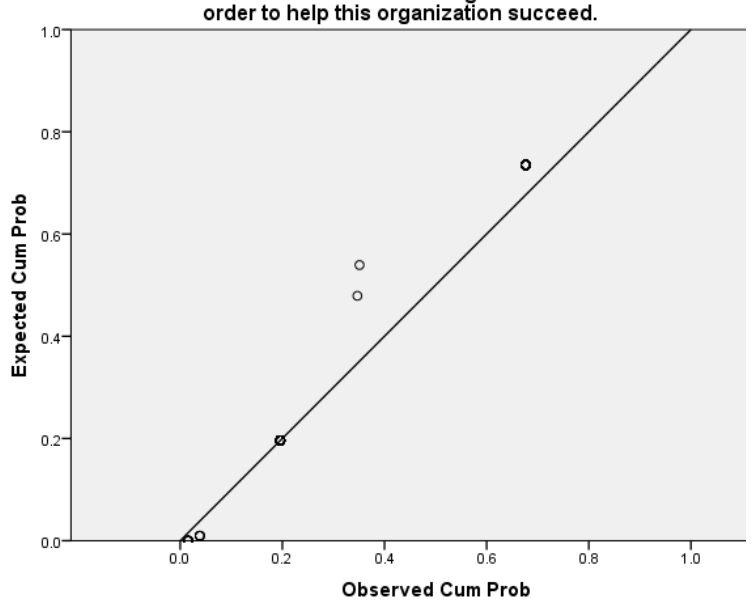

OC1 

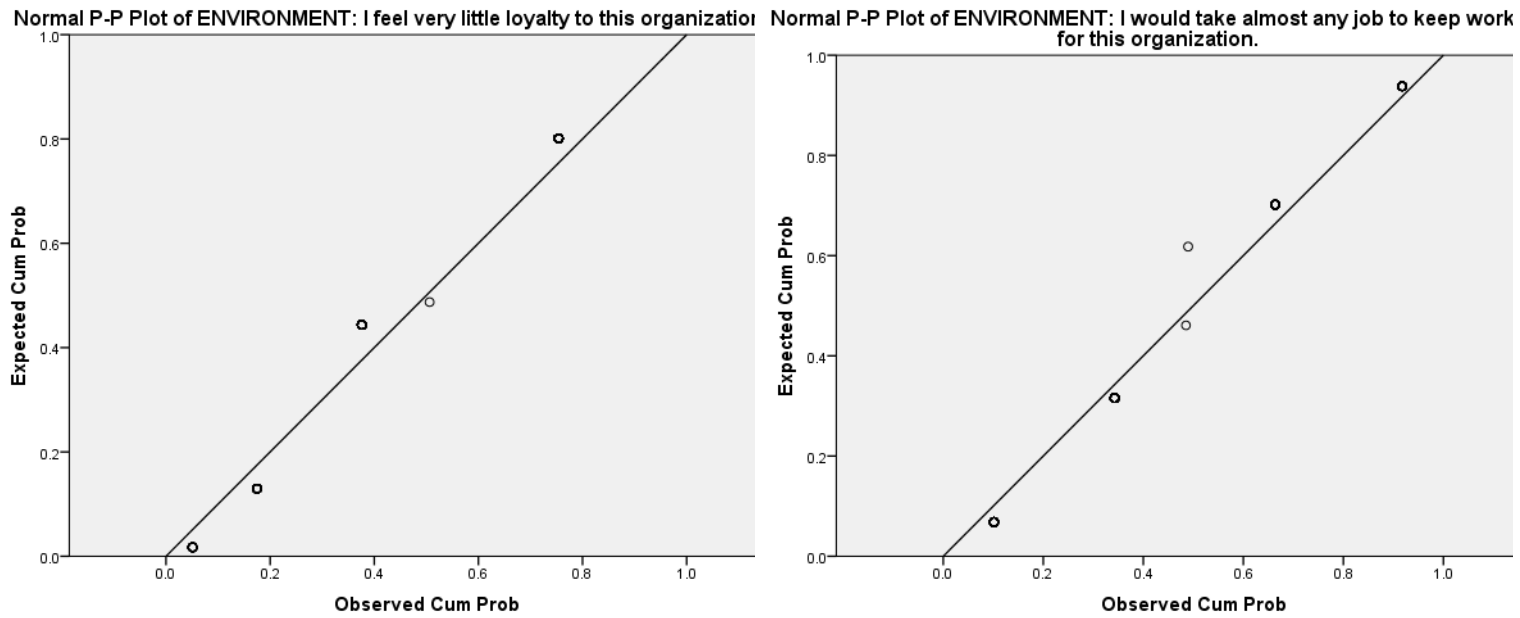

OC2

\section{$\mathrm{OC} 3$}

Normal P-P Plot of ENVIRONMENT:I find that my values and the agency's

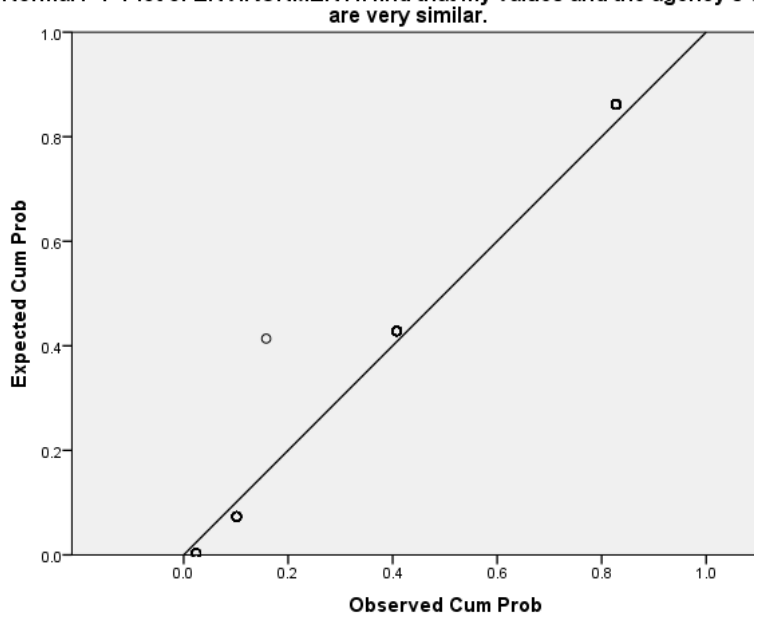

Normal P.P Plot of ENVIRONMENT: I am proud to be working for this

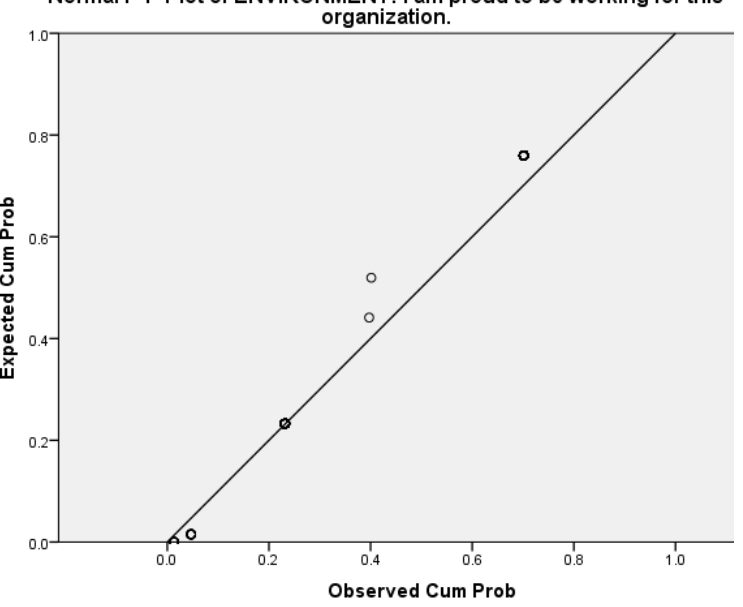

\section{OC4}

\section{OC5}

Normal P-P Plot of ENVIRONMENT: I would turn down another job for more |
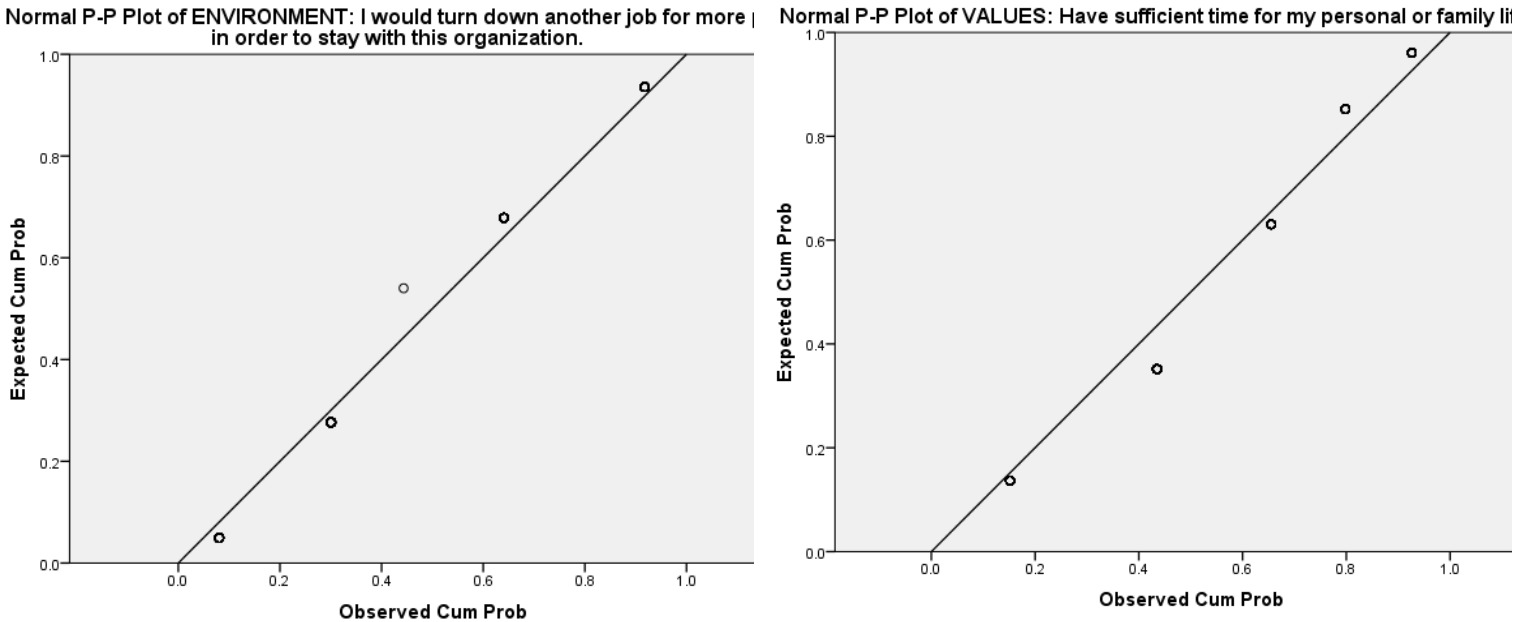

OC6

CV1 
Normal P-P Plot of VALUES: Have good physical working conditions (goo

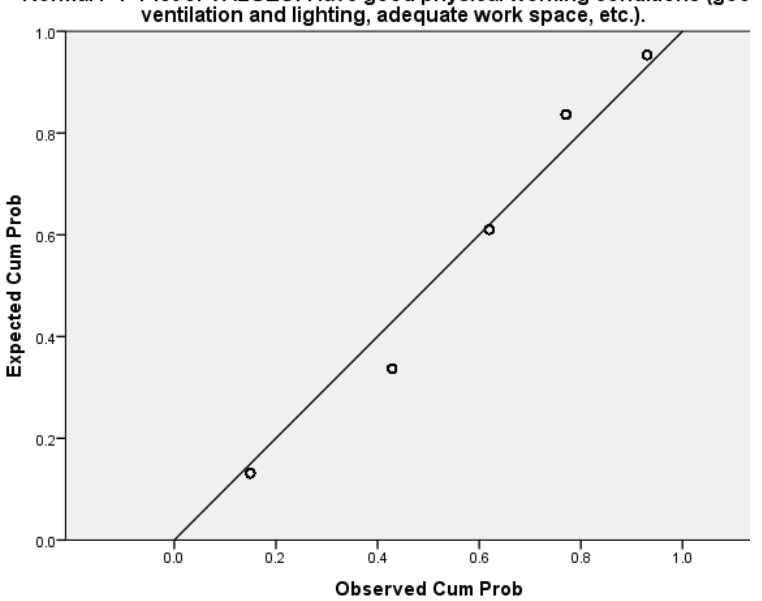

CV2

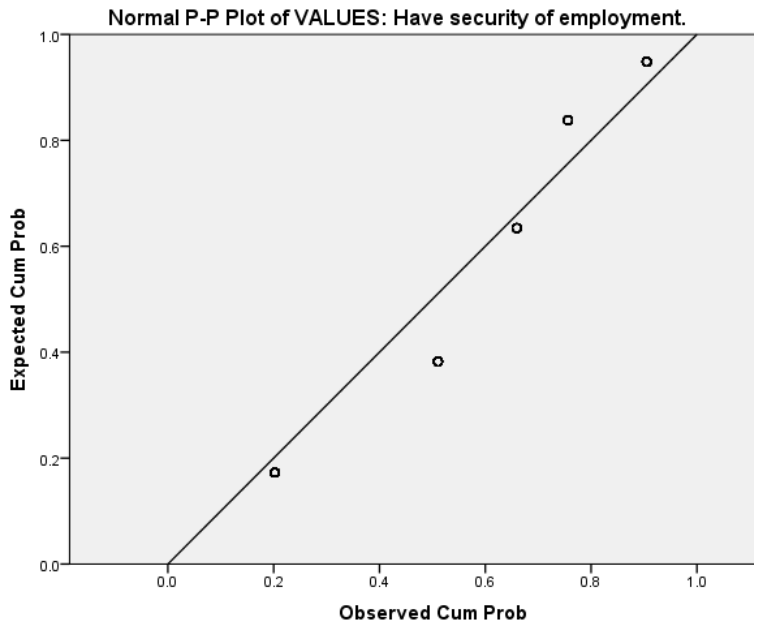

CV4

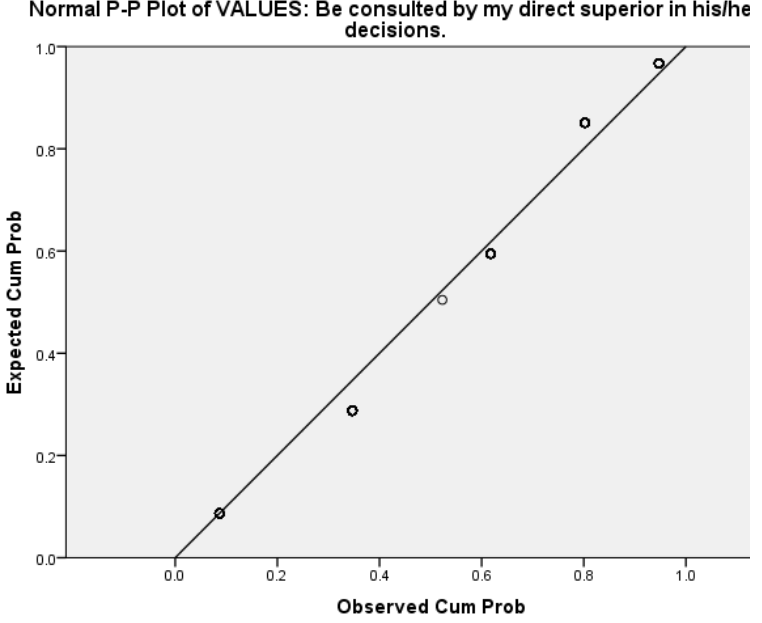

CV6

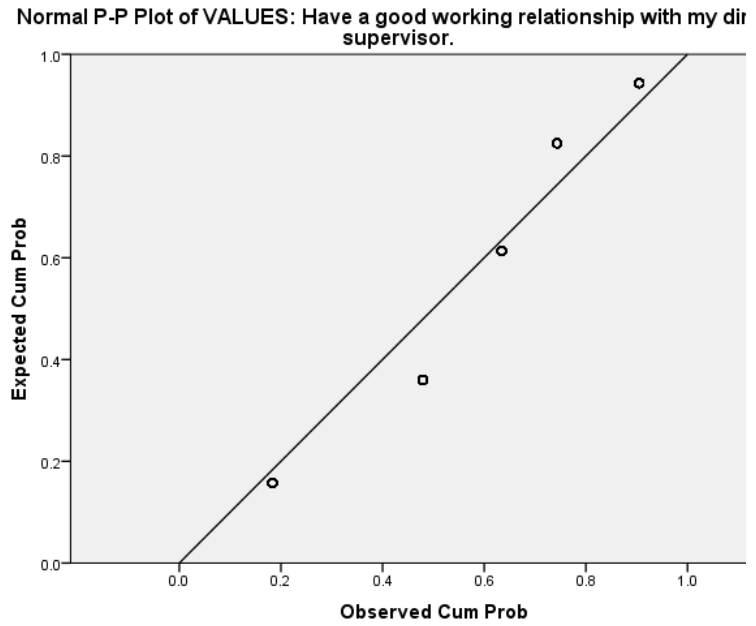

CV3

Normal P-P Plot of VALUES: Work with people who cooperate well with o

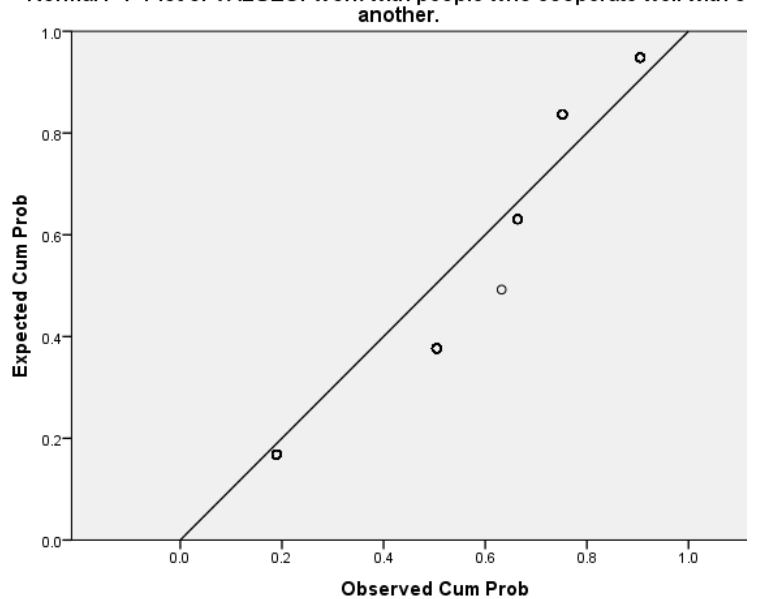

CV5

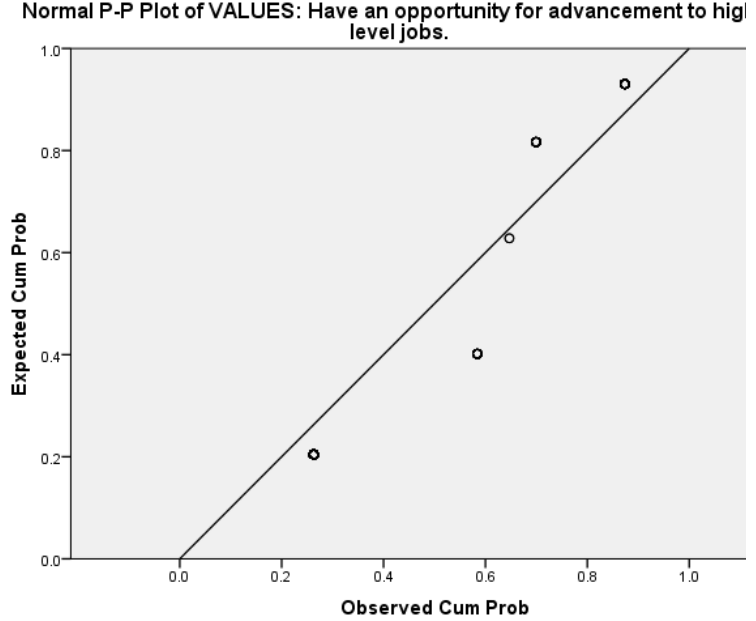

CV7 

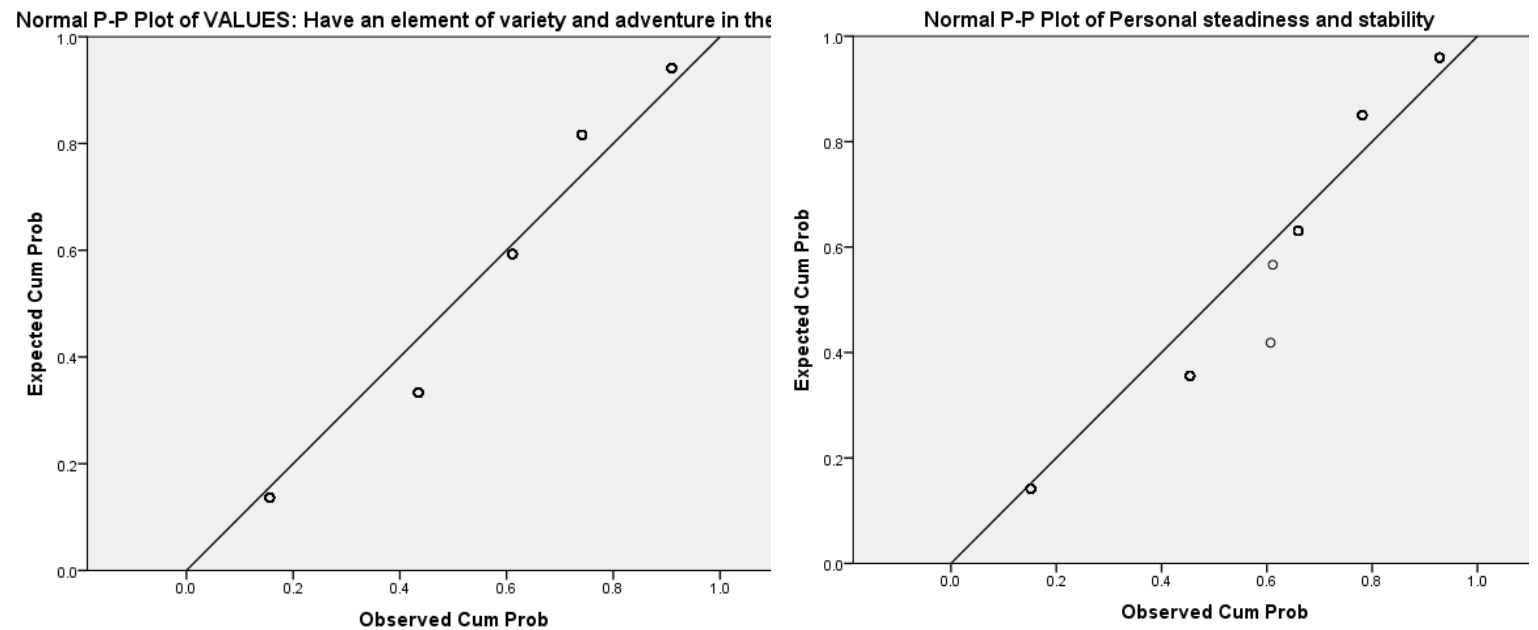

\section{CV8}

\section{CV9}
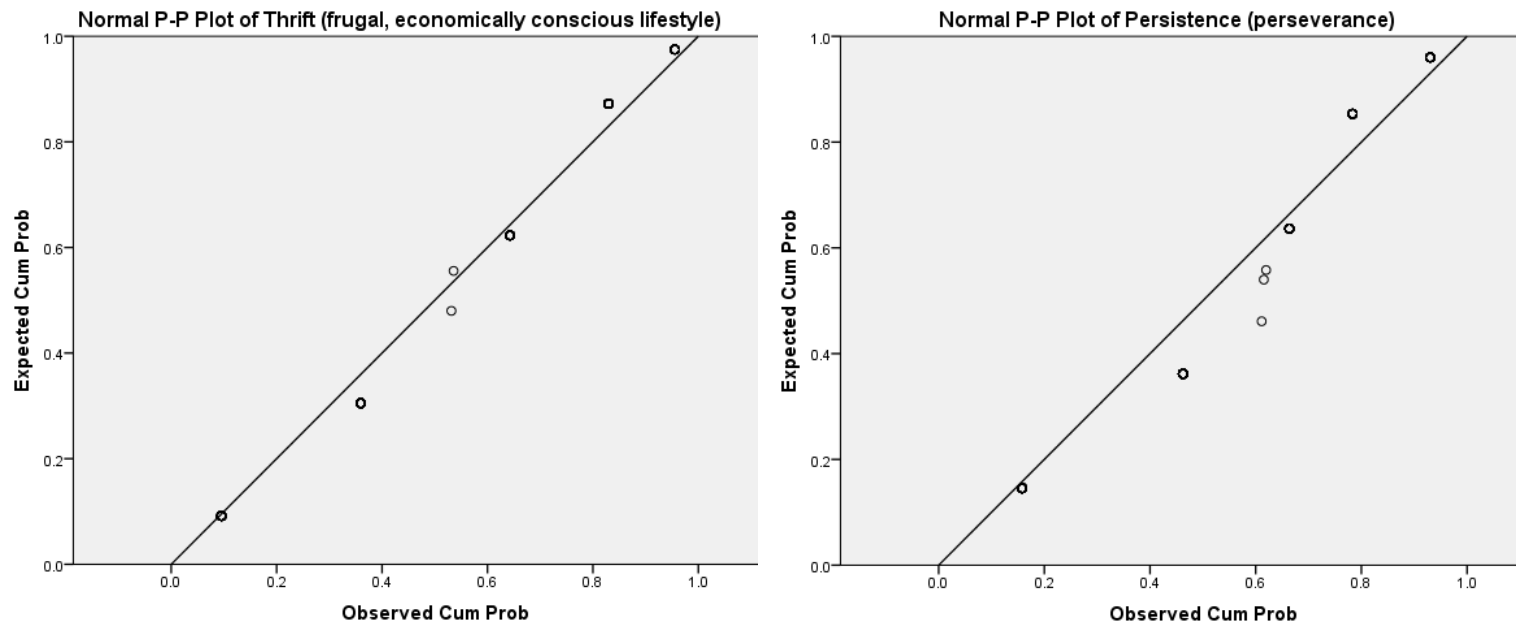

\section{CV10}

CV11
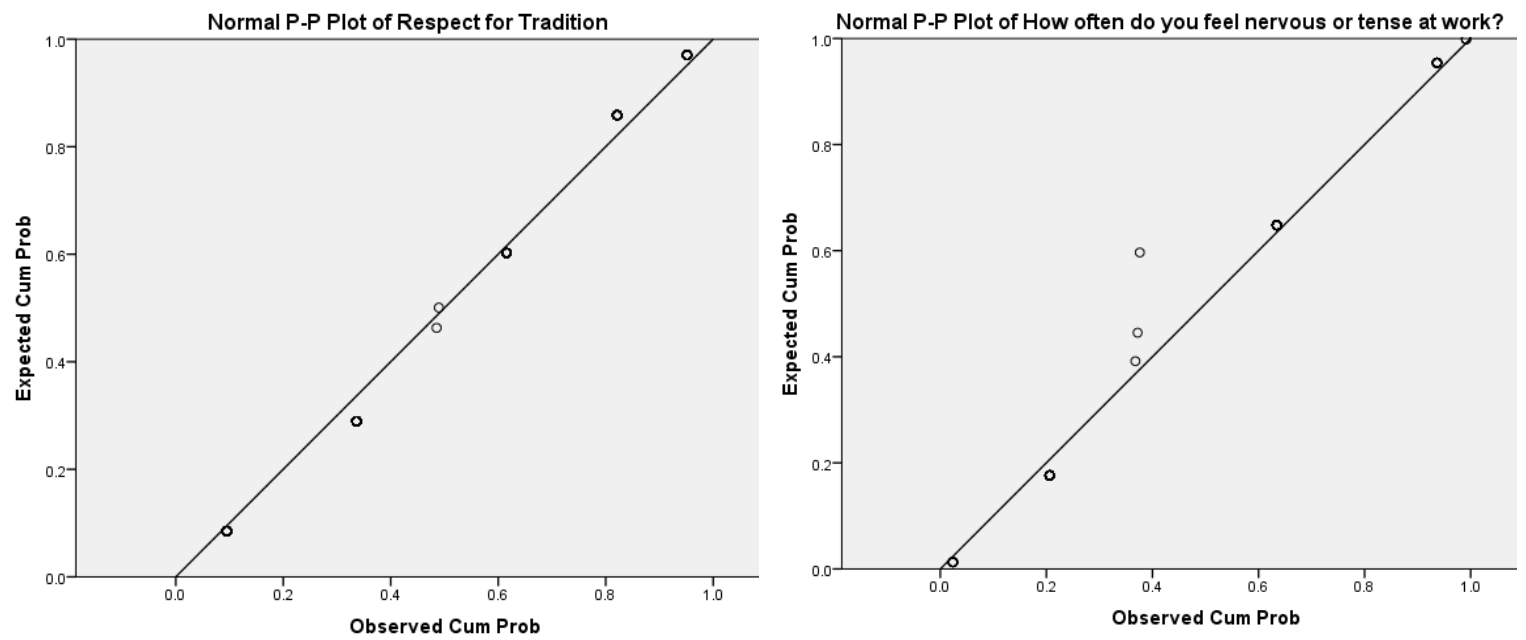

CV12

\section{CV13}


Normal P-P Plot of How frequently, in your experience, are subordinates afrai
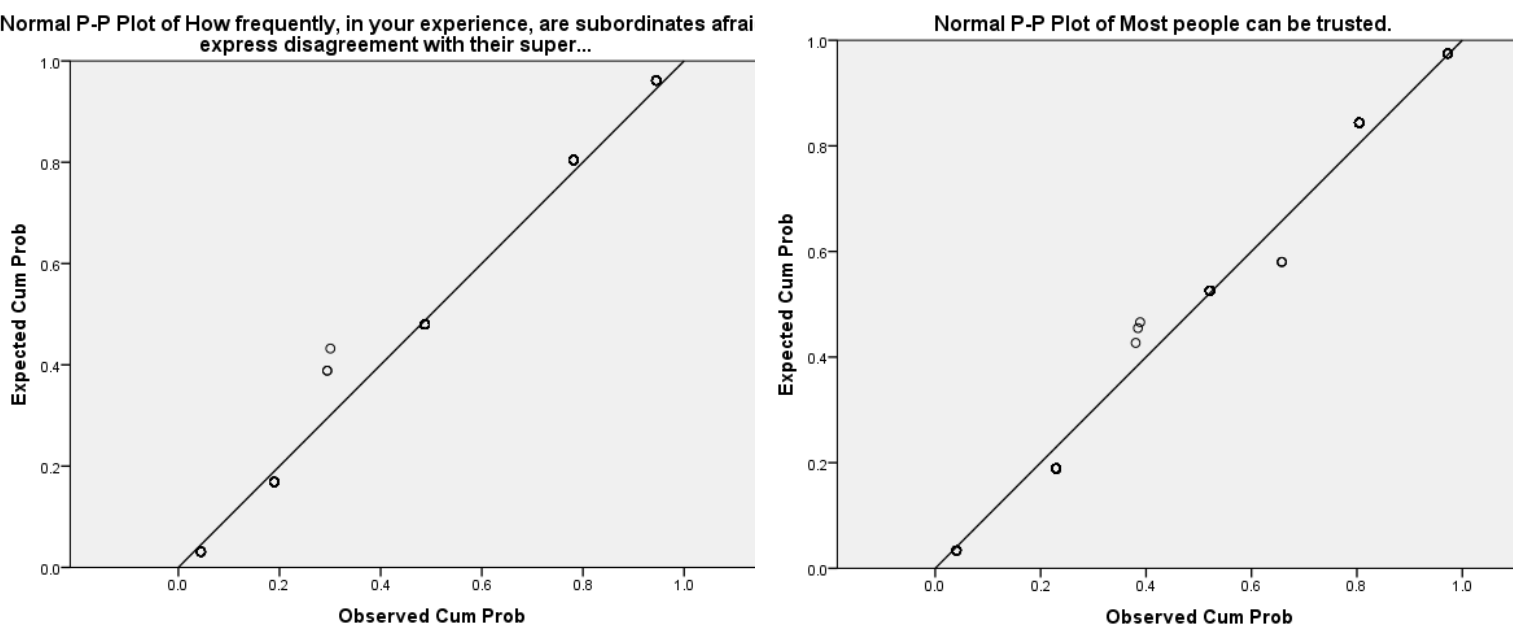

\section{CV14}

\section{CV15}
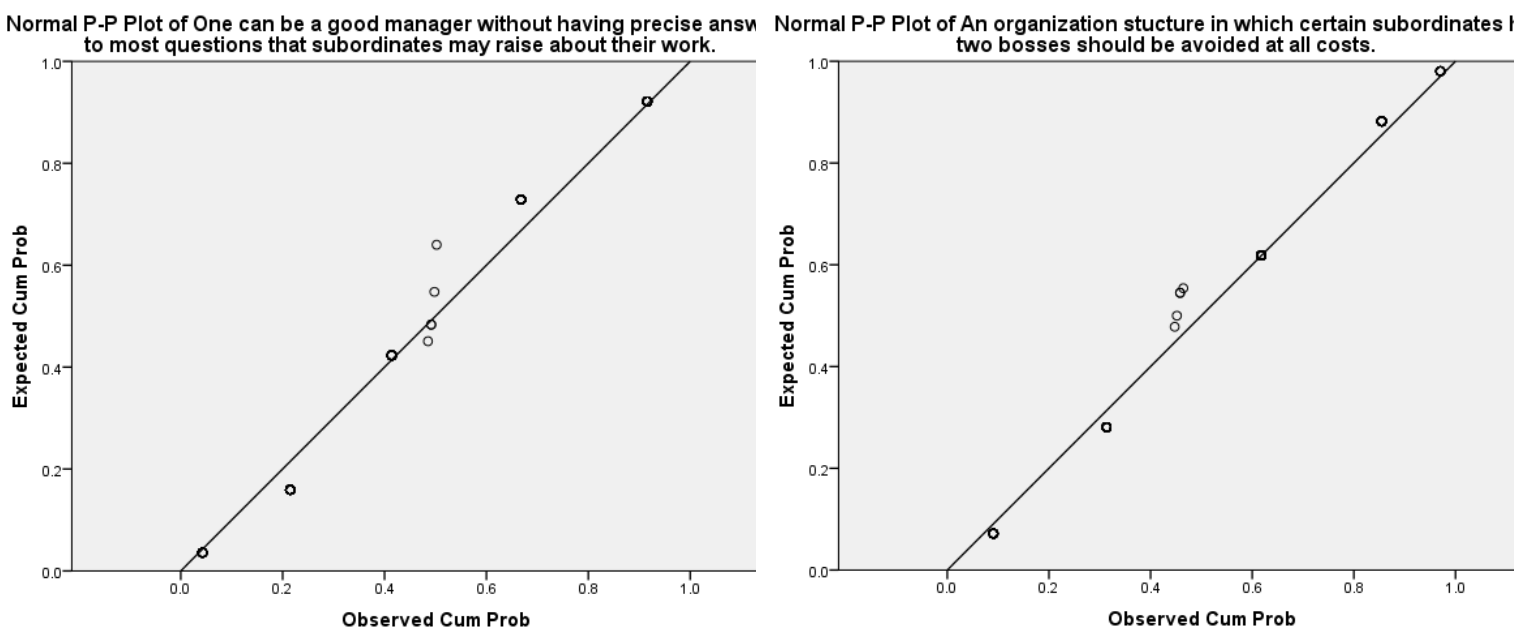

CV16

CV17

Normal P.P Plot of Competition between empoyees usually does more harm good.
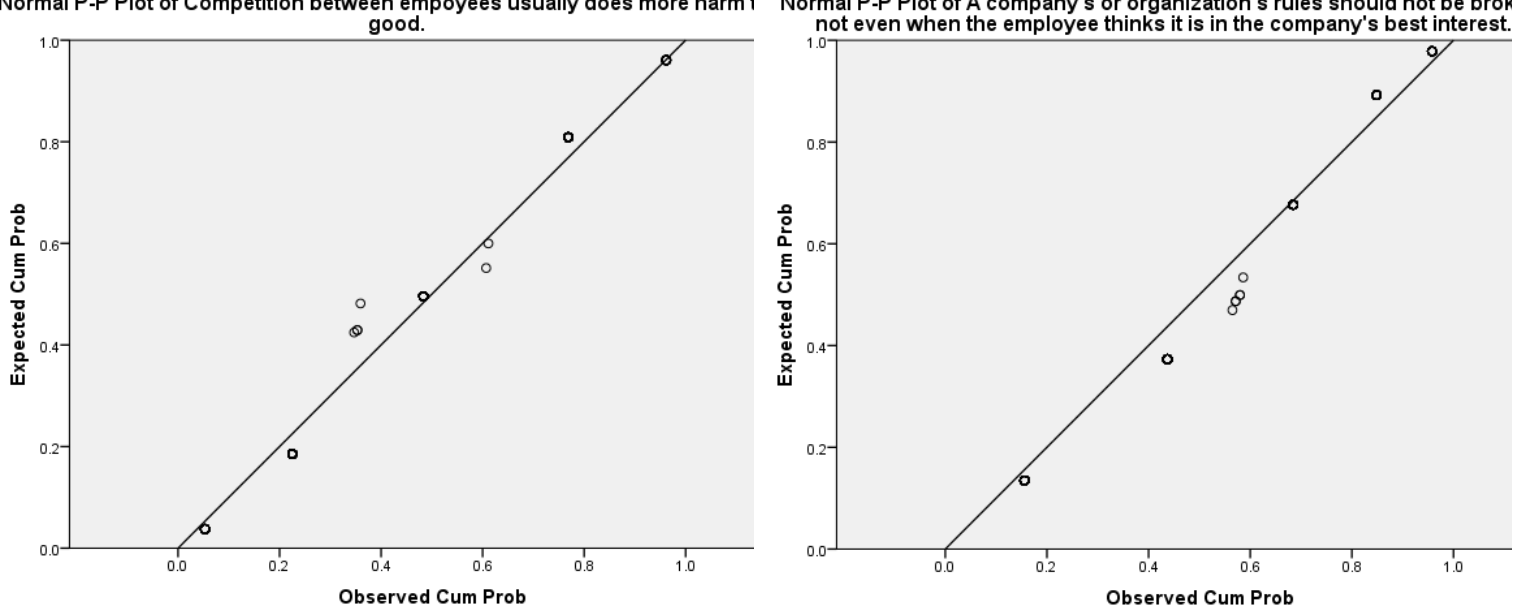

CV18

CV19 
Normal P-P Plot of When people have failed in life it is often their own faul

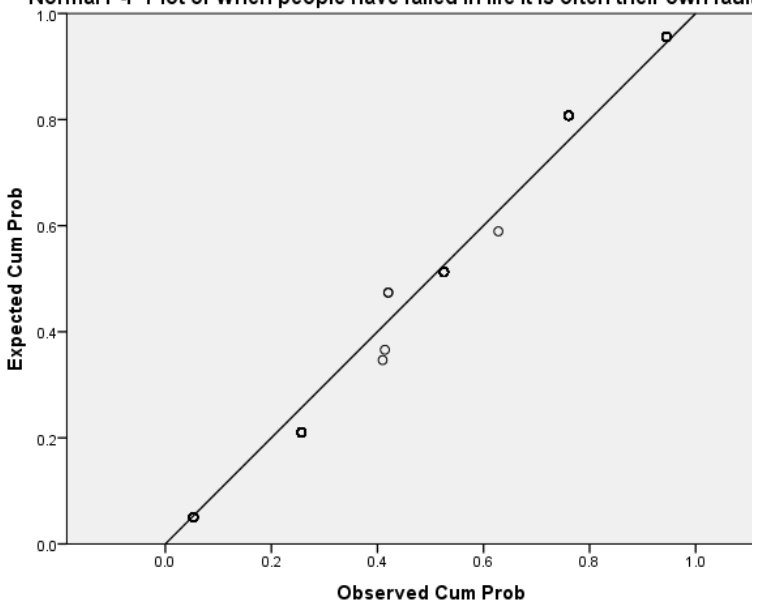

CV20 


\section{APPENDIX 8}

\section{Consolidated Results - Histograms and Box Plots by Hemisphere}

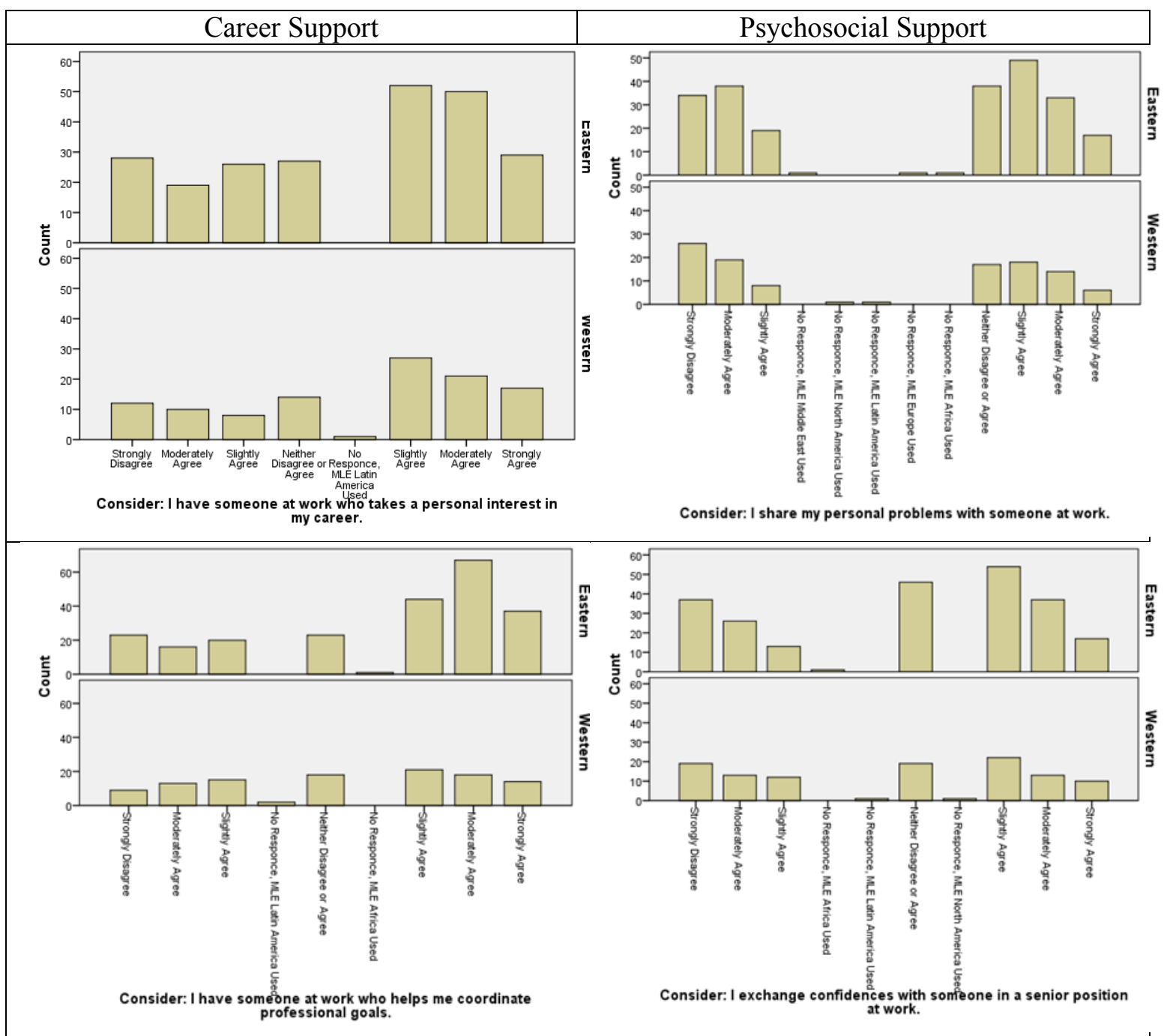



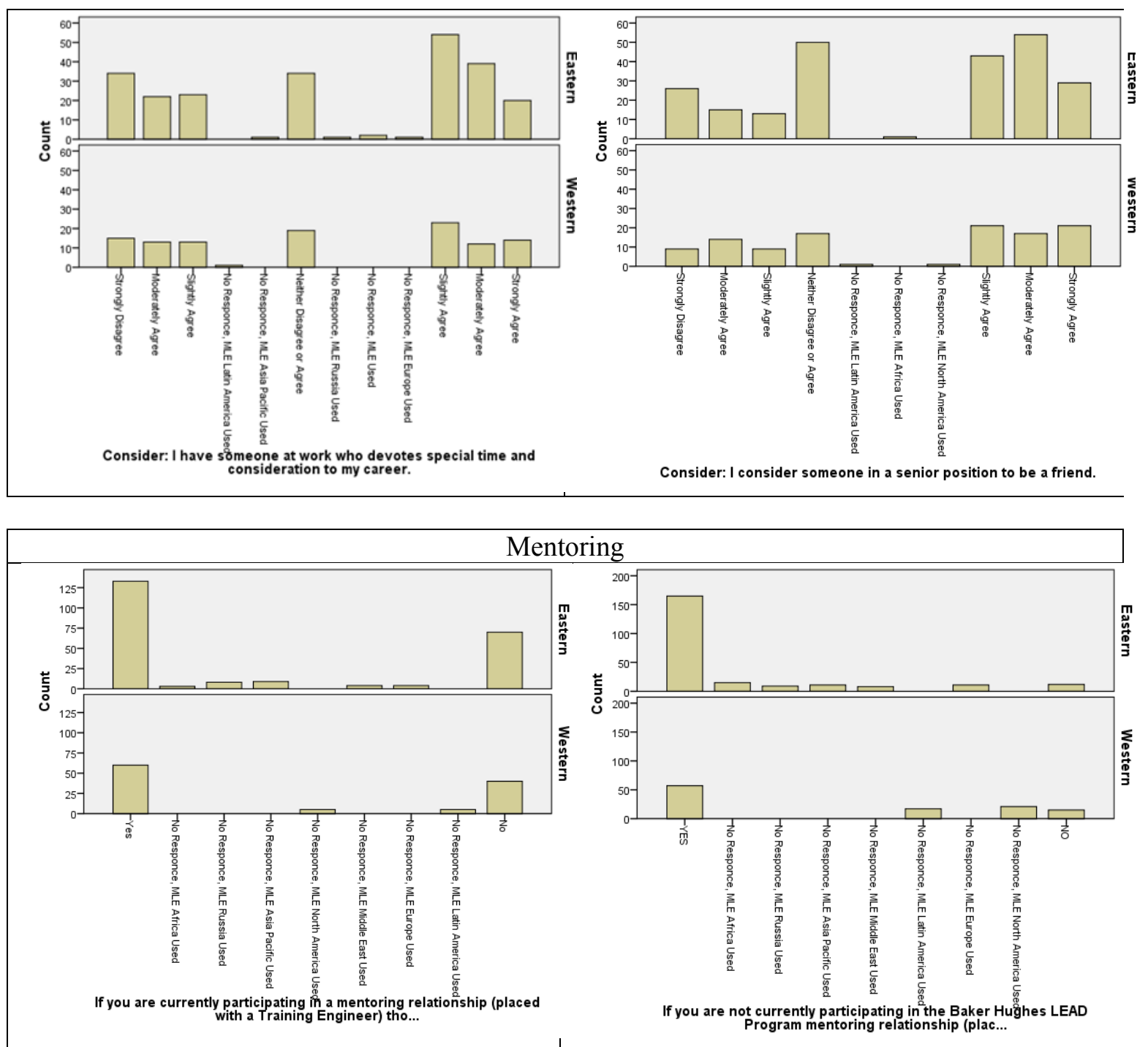


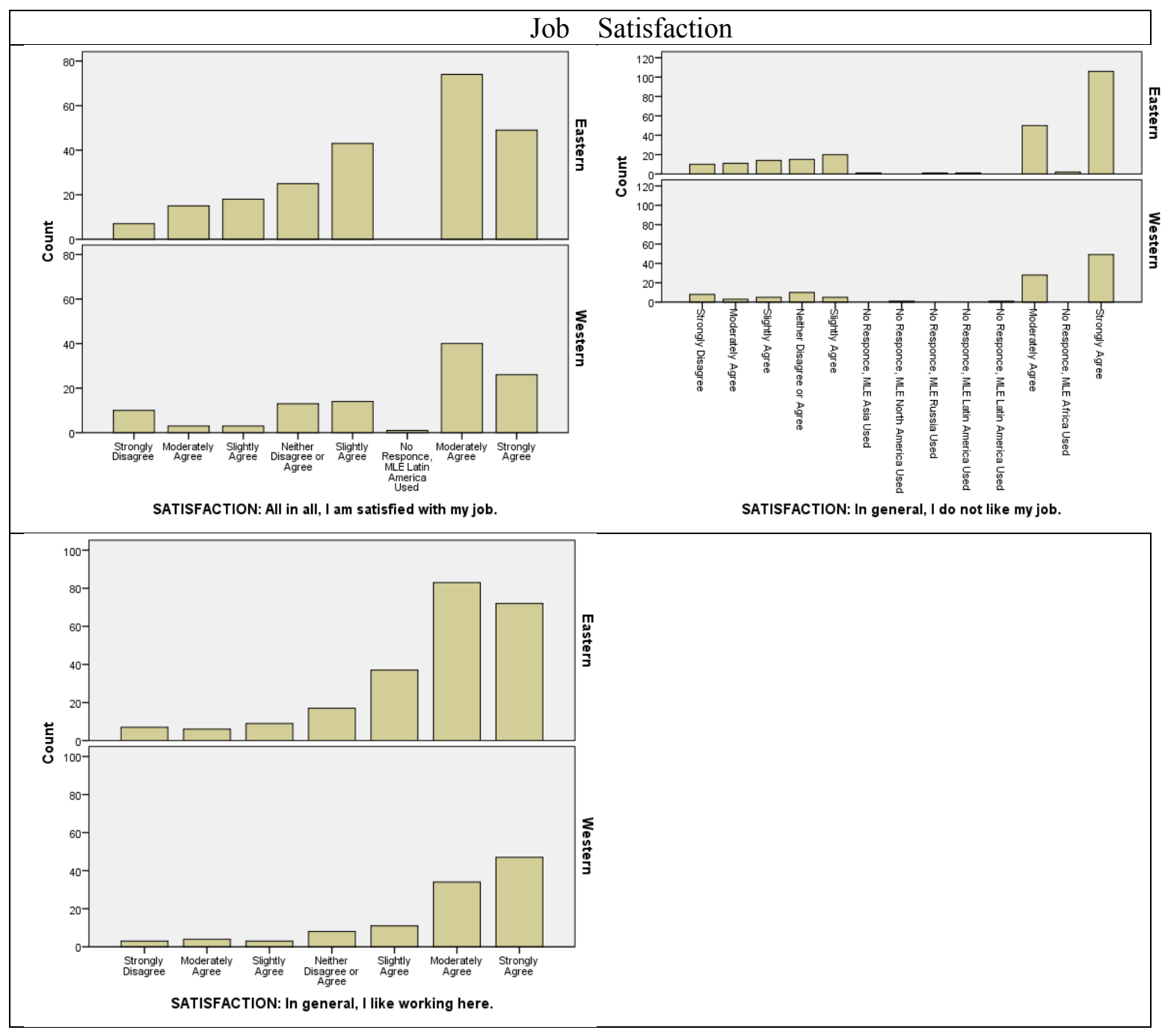




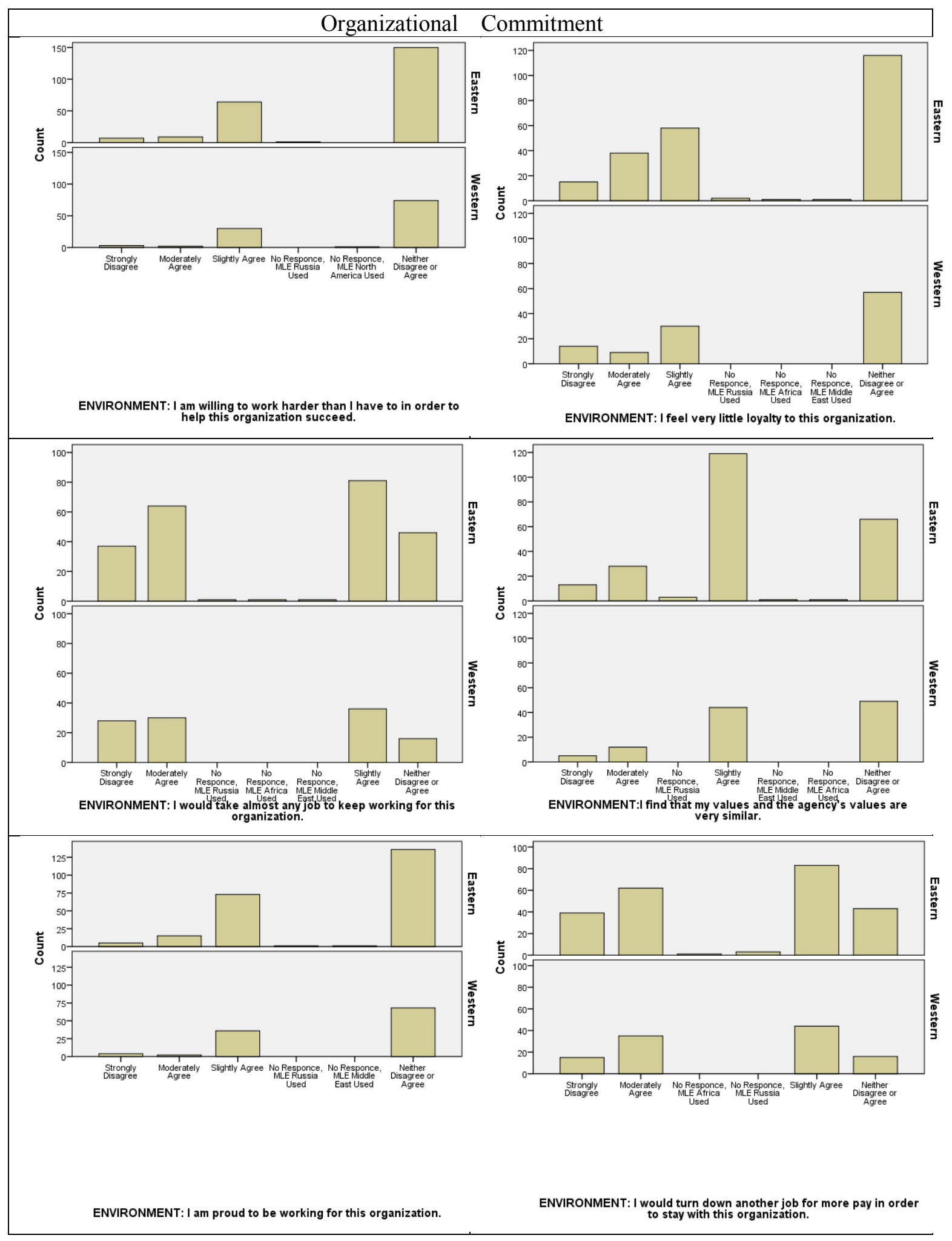




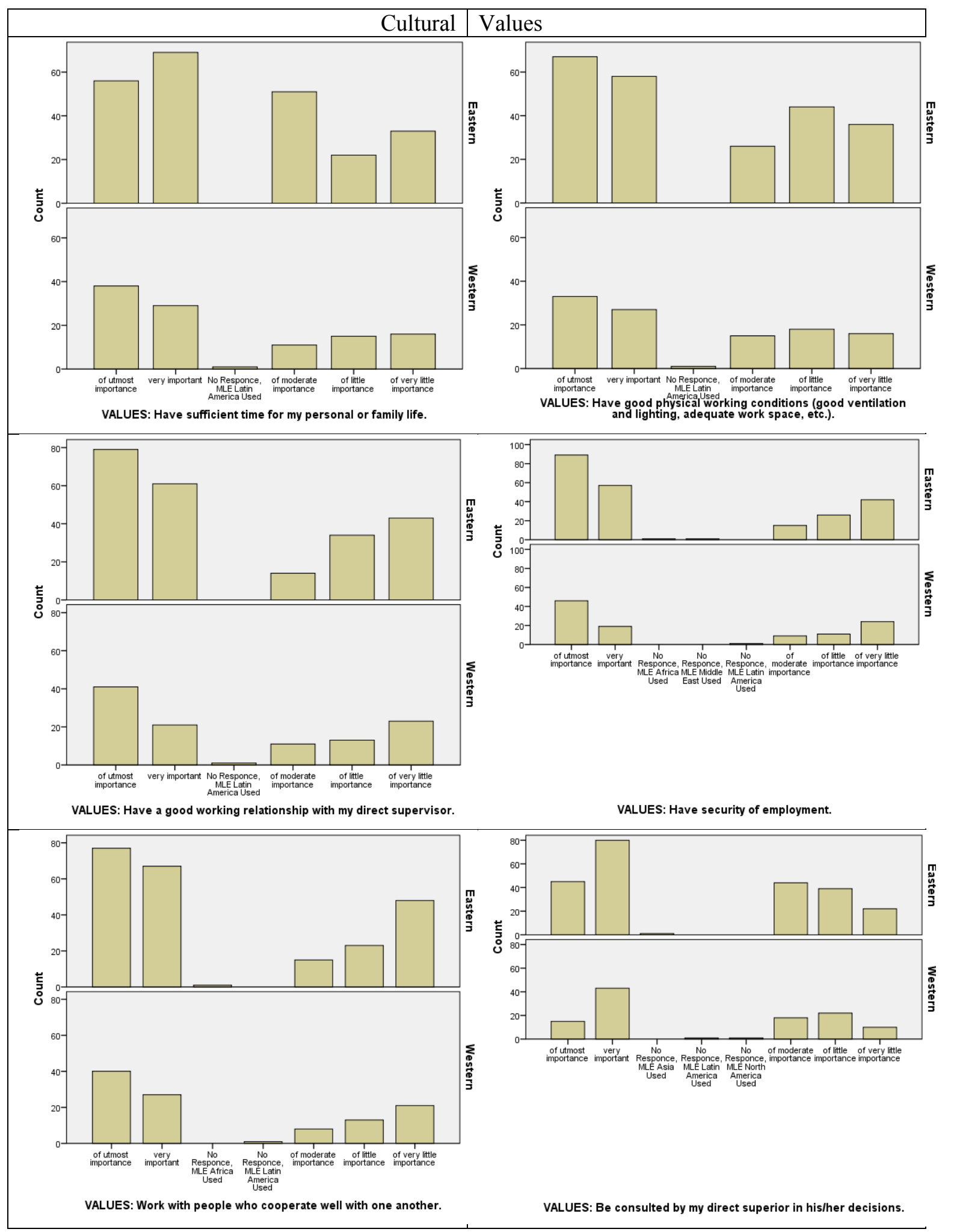




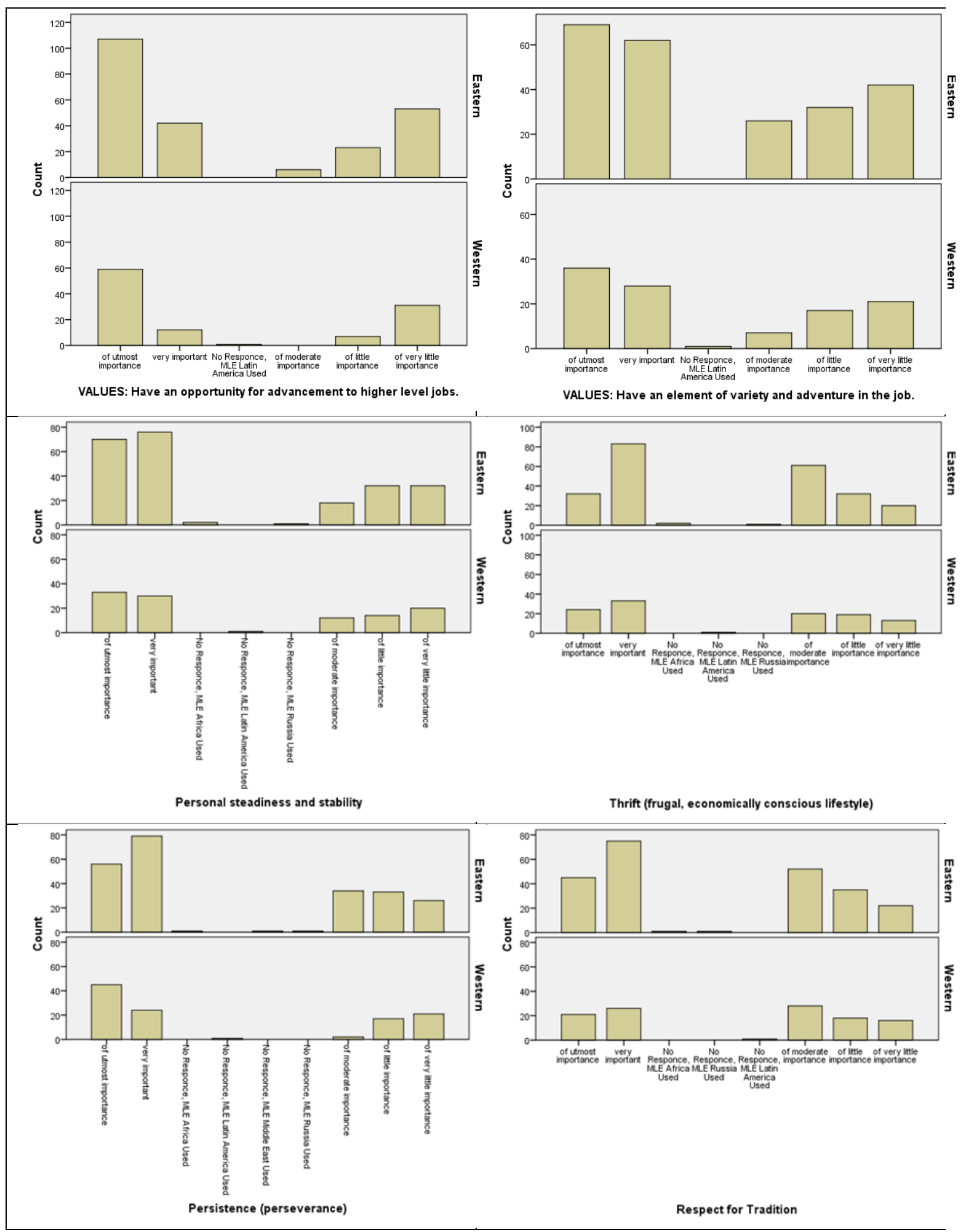




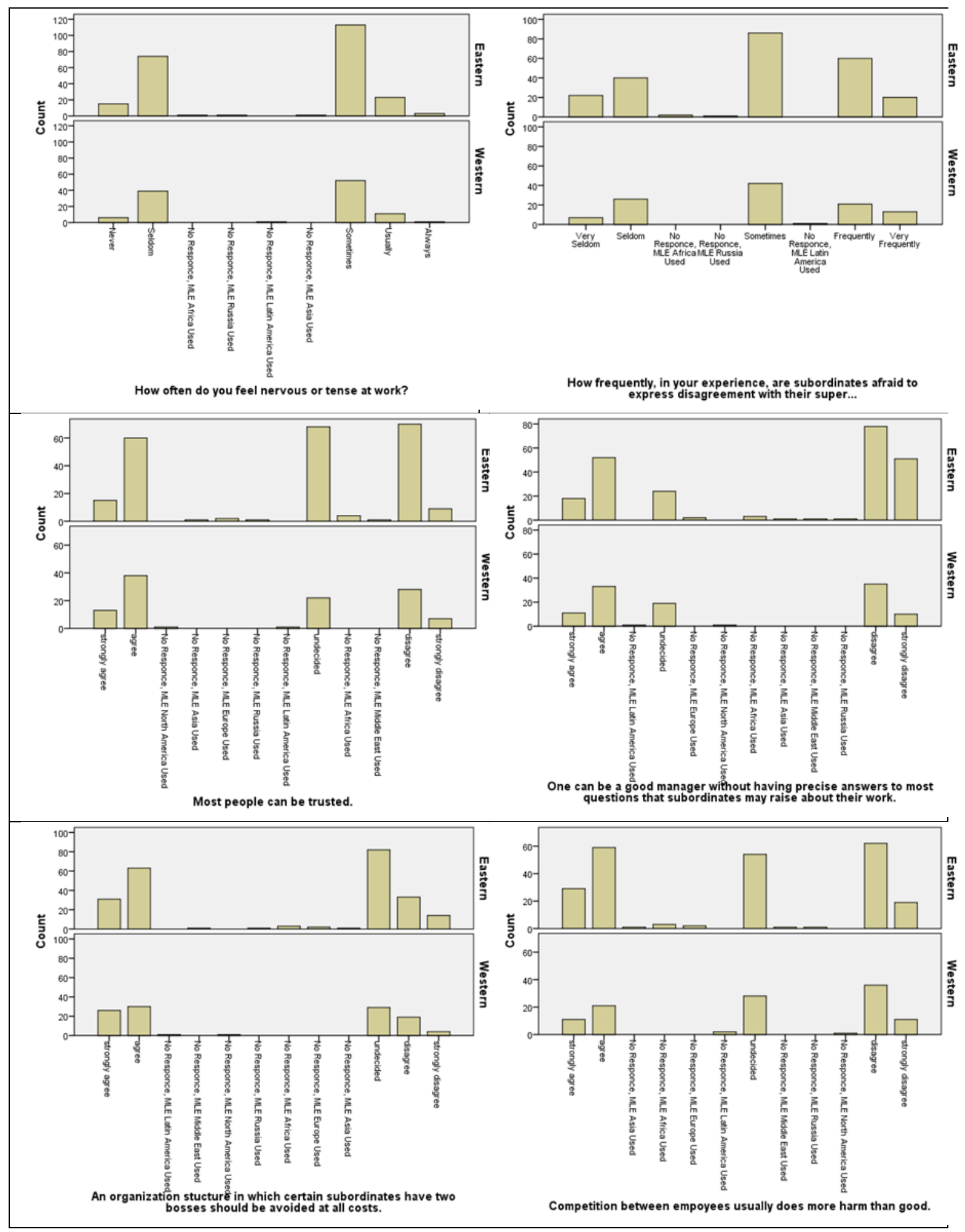




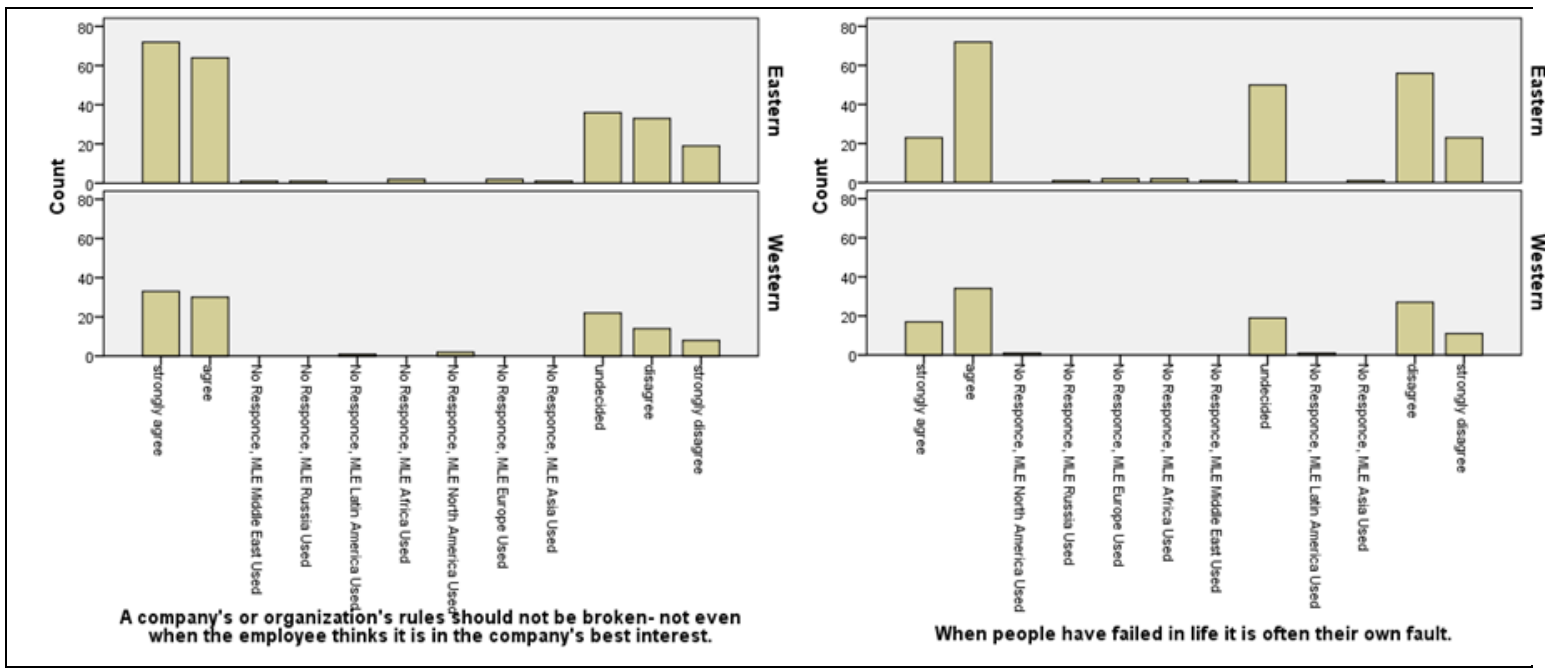




\section{APPENDIX 9}

Consolidated Results - Histograms and Boxplots by Region

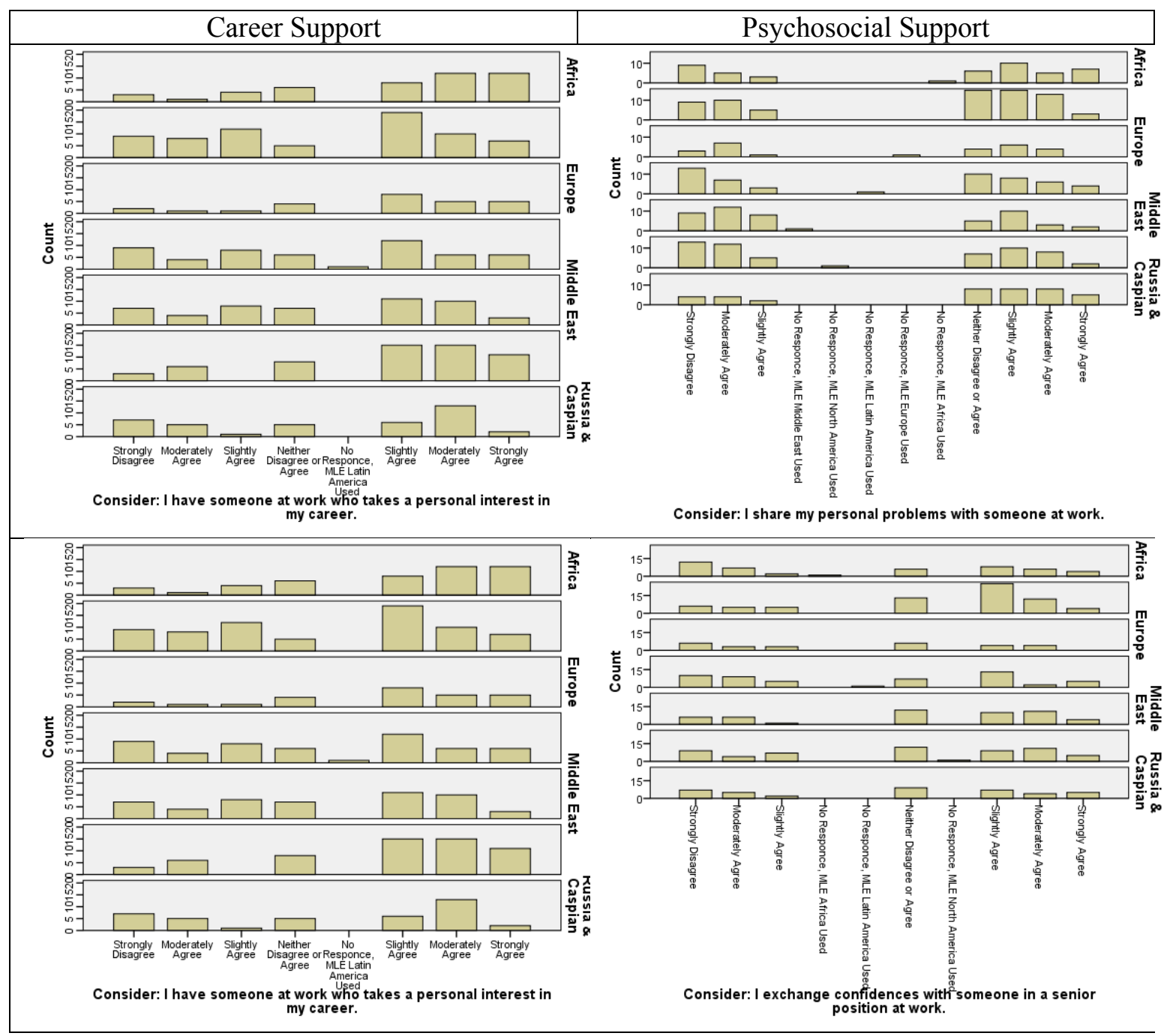



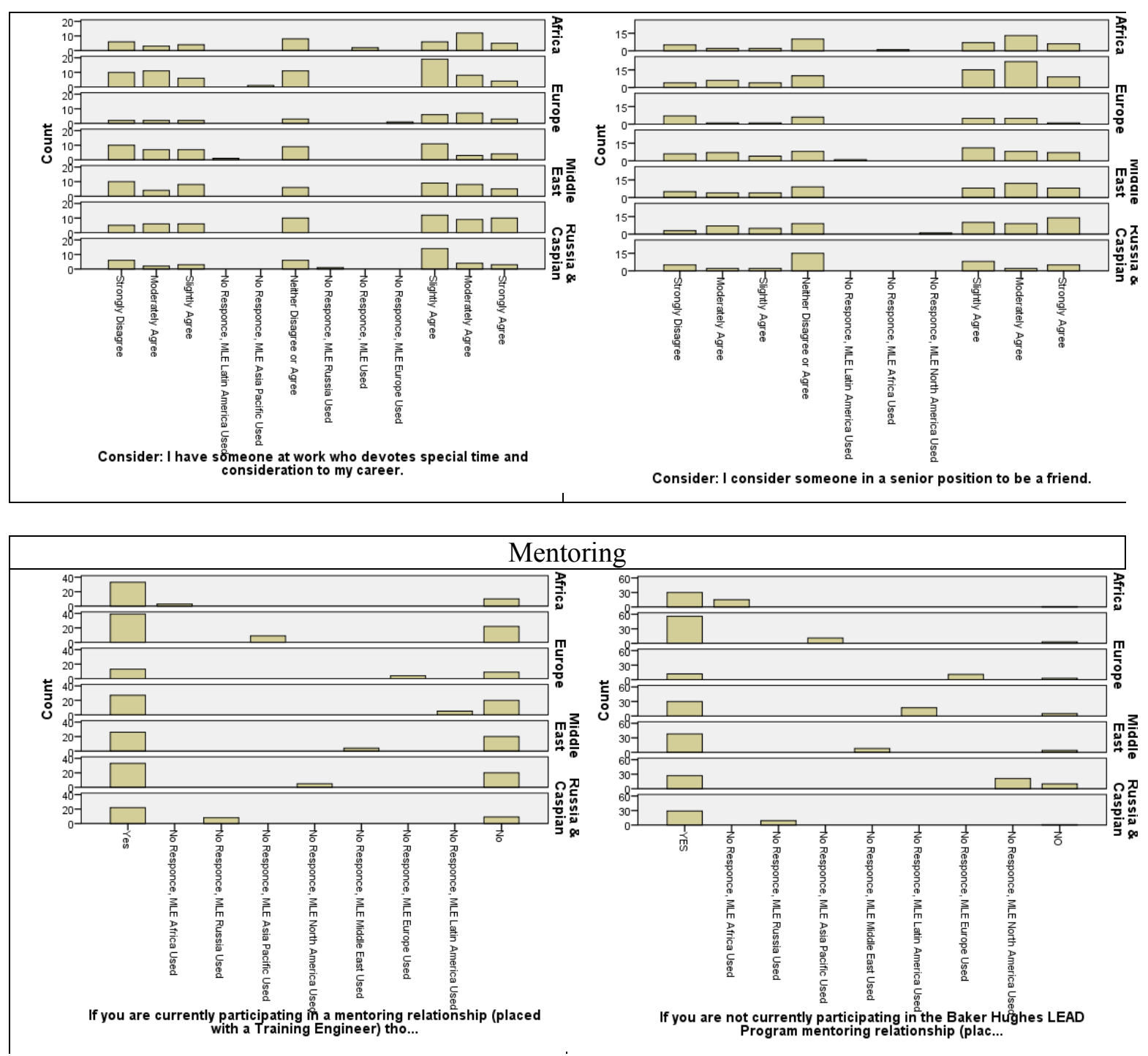


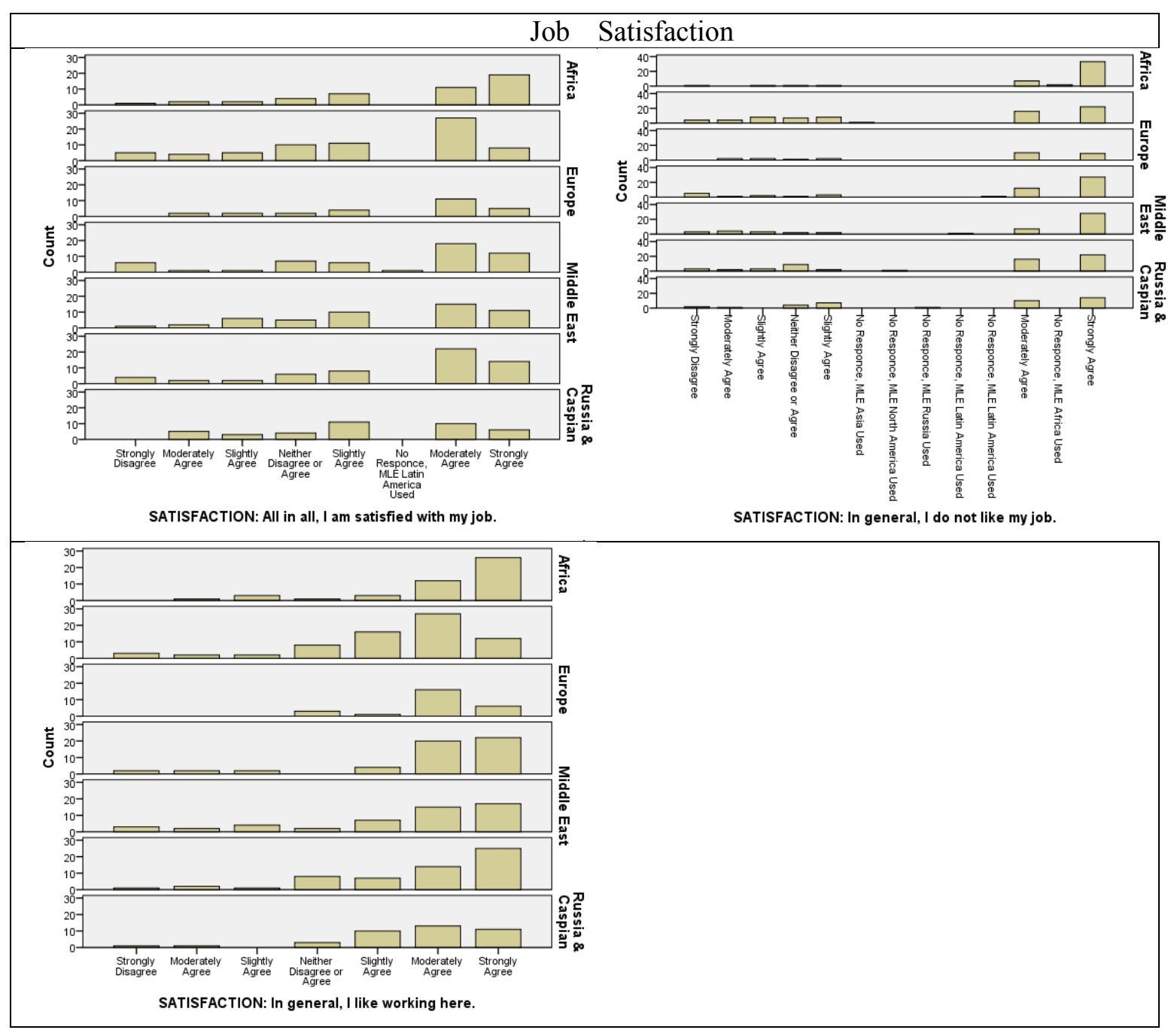




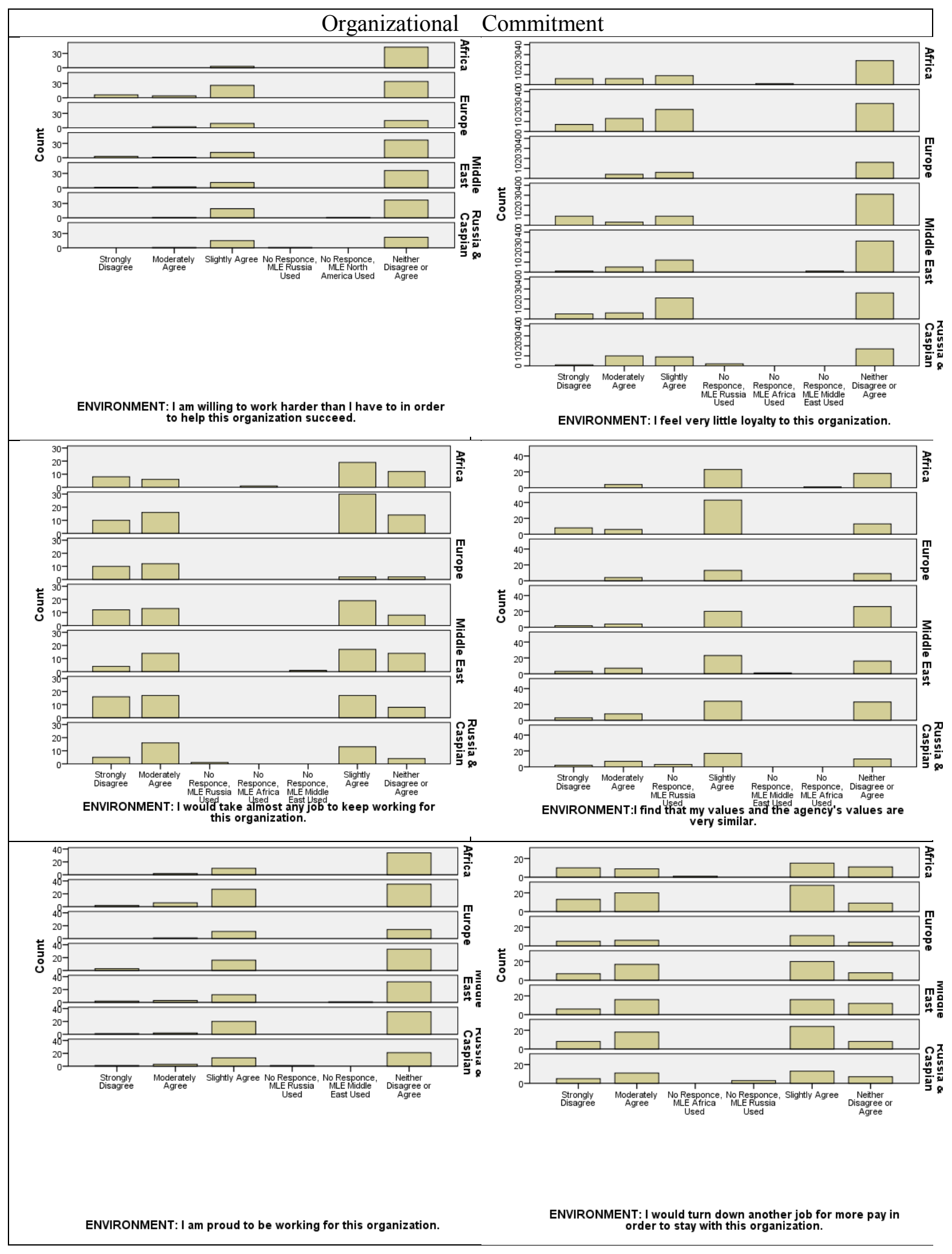




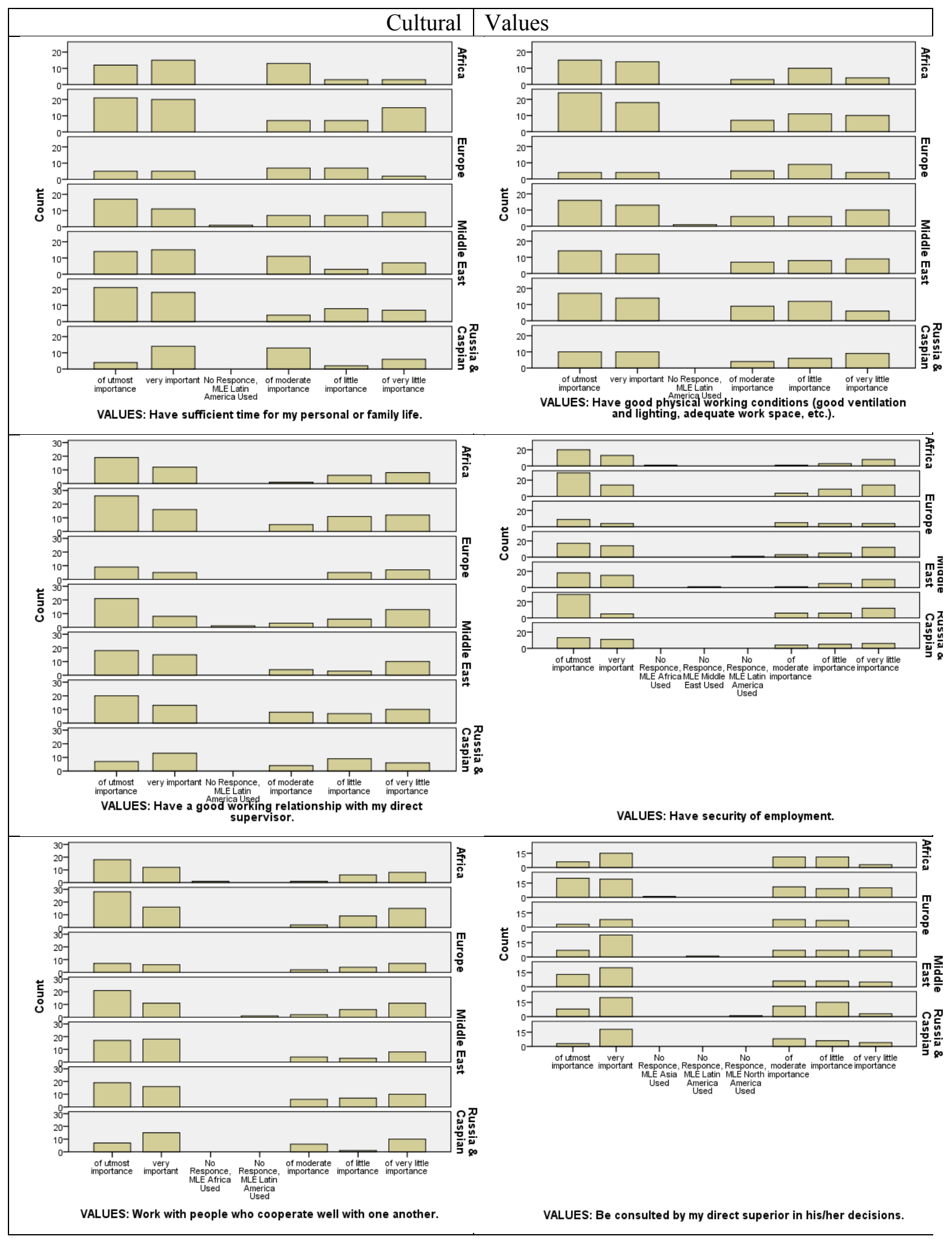




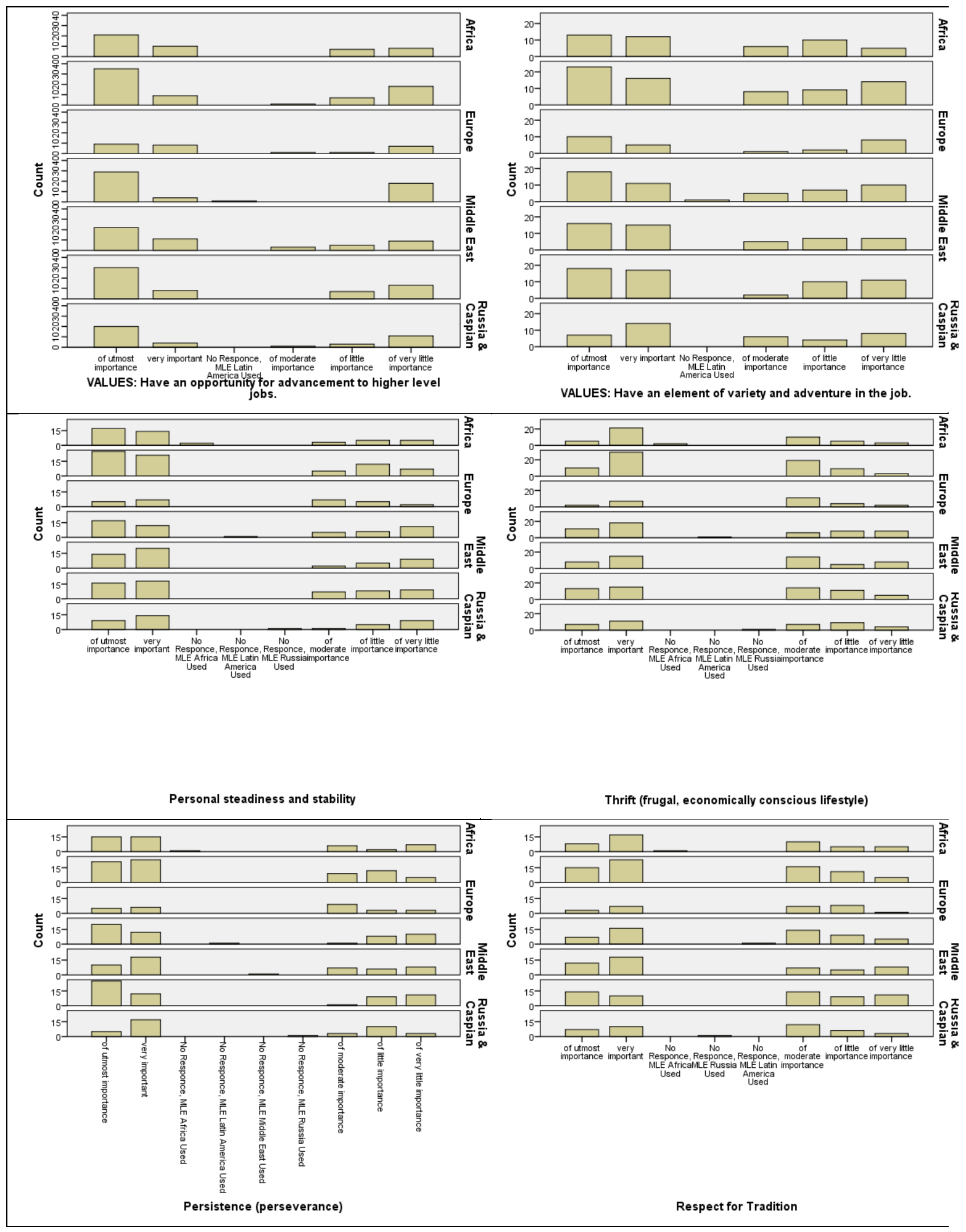



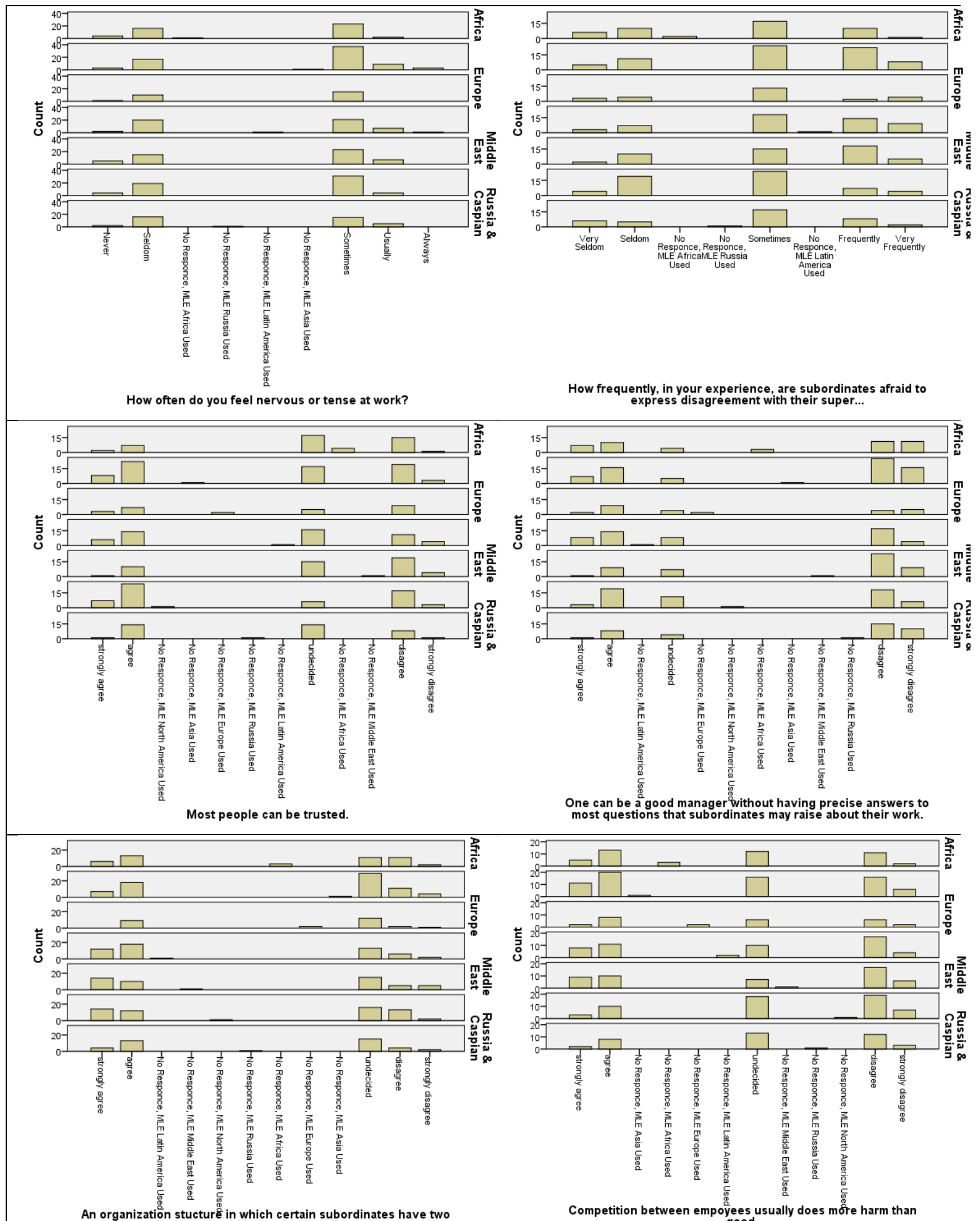

most questions that subordinates may raise about their work.

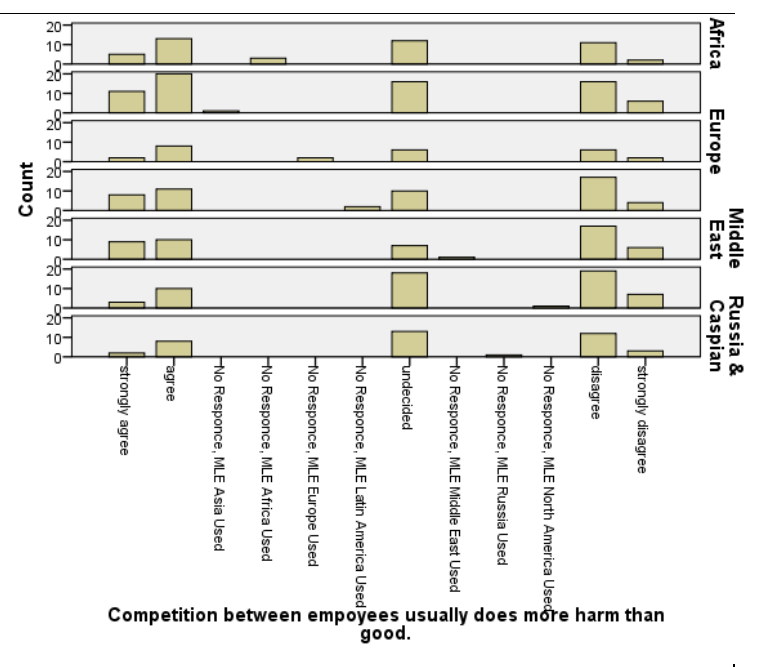




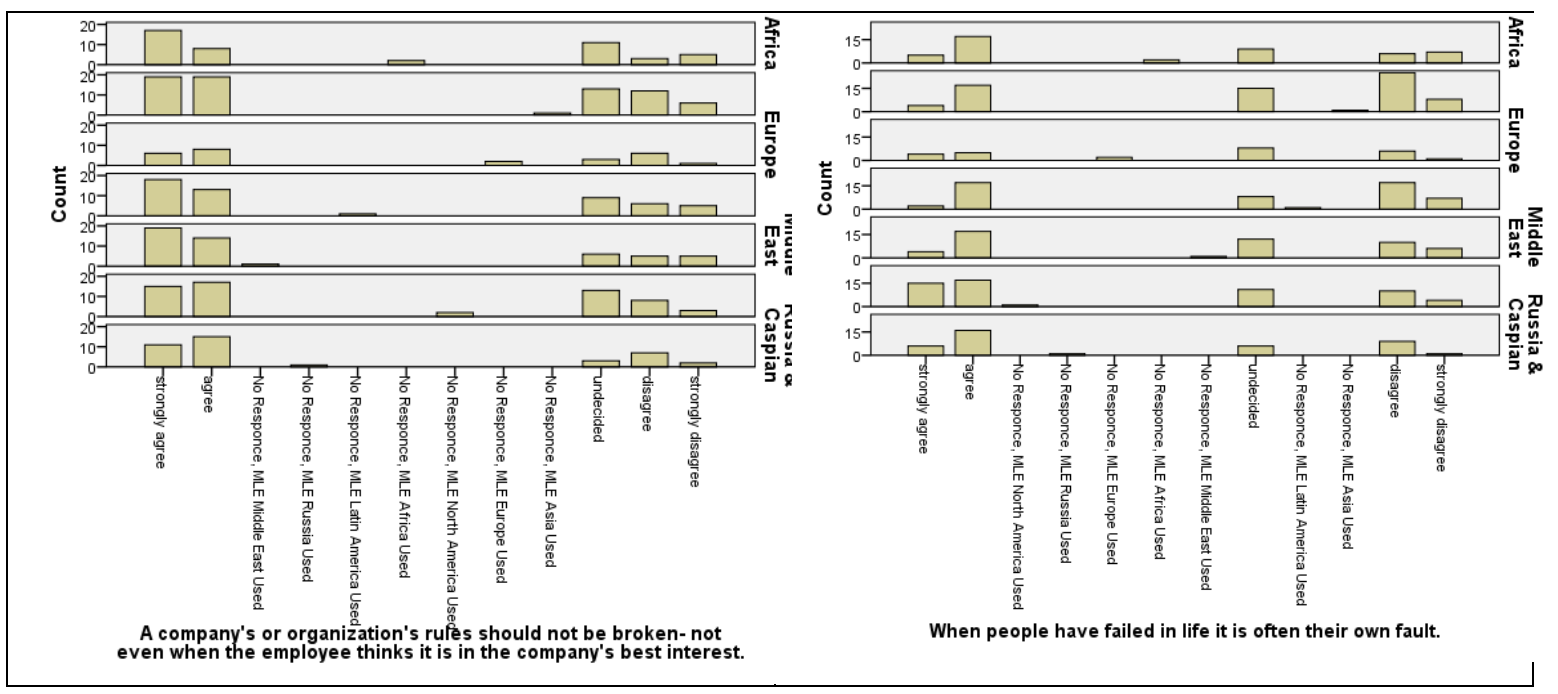




\section{APPENDIX 10}

Hypothesis IV - Demographic Breakdown - Summary and Regional Structural Models for Perceived Organizational Support and Affective Reaction

Due to properties of the smaller data set, estimated means and intercepts were used which negates the GFI test statistic.

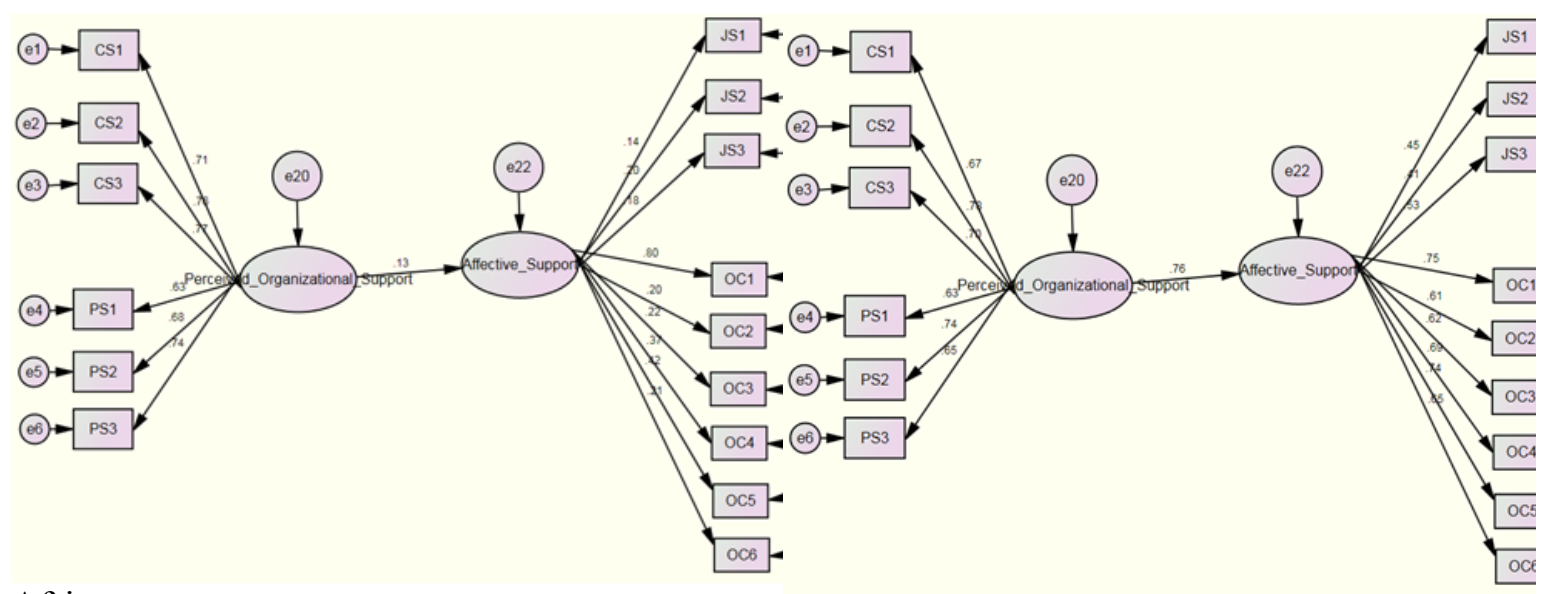

Africa

Asia Pacific

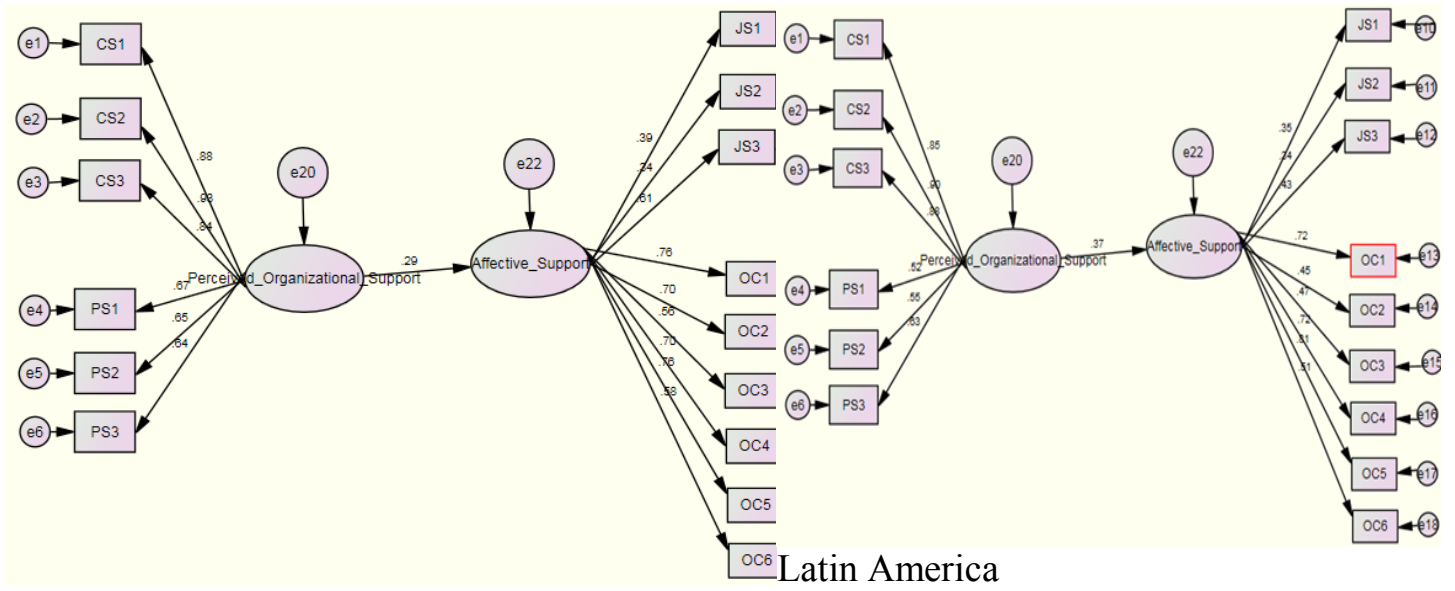

Europe 


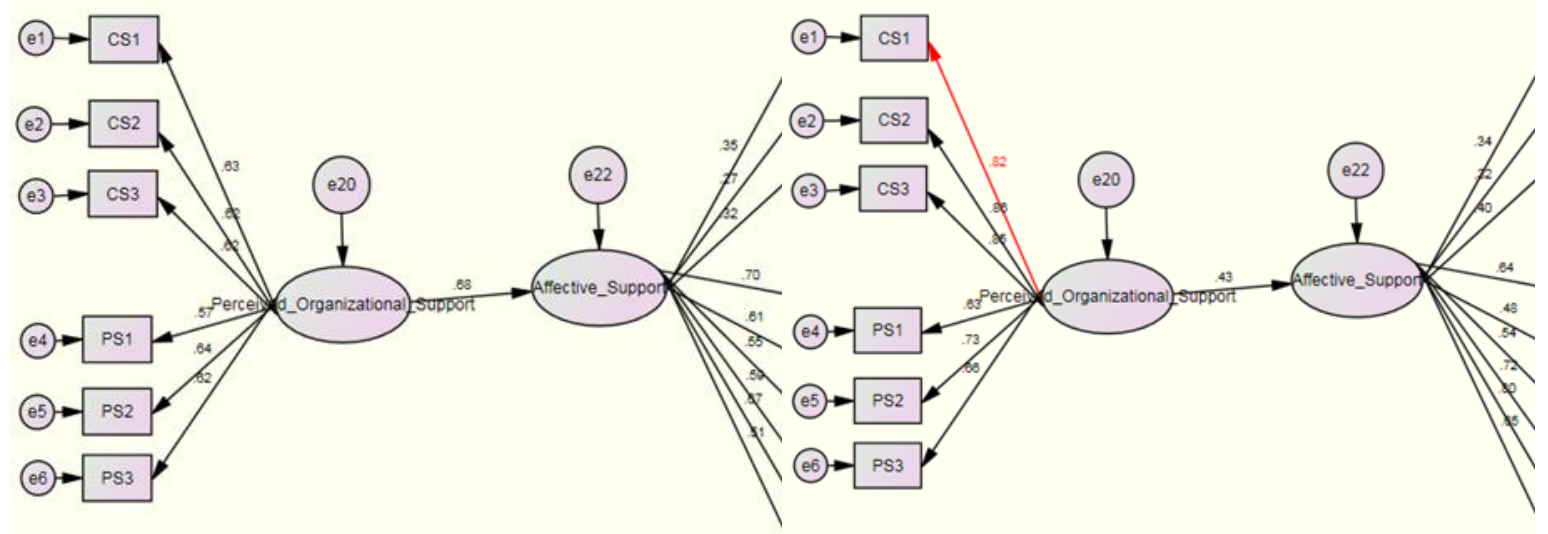

Middle East

North America

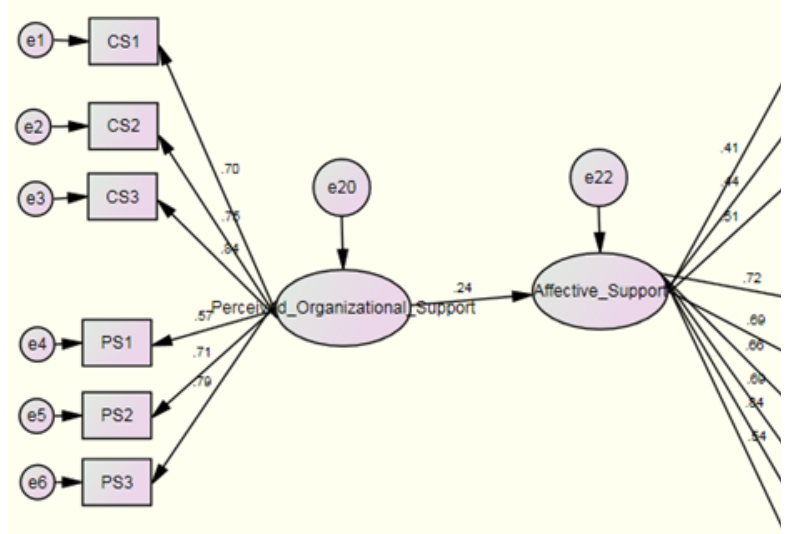

Russia \& Caspian

\section{Summary of Model Fit Indices Perceived Organizational Support and Affective Reaction}

\begin{tabular}{|l|c|c|}
\hline Model & NFI & CFI \\
\hline Africa & 0.458 & 0.683 \\
\hline Asia Pacific & 0.566 & 0.674 \\
\hline Europe & 0.407 & 0.575 \\
\hline Latin America & 0.330 & 0.411 \\
\hline Middle East & 0.422 & 0.575 \\
\hline North America & 0.500 & 0.625 \\
\hline Russia \& Caspian & 0.478 & 0.648 \\
\hline
\end{tabular}

Neither the NFI nor CFI are at sufficient level as to suggest a good model. This is to be expected as these are subsamples of a skewed distribution. 
Summary Table for Perceived Organizational Support and Affective Reaction

\begin{tabular}{|l|c|c|}
\hline & r & $\begin{array}{c}\text { Support } \\
\text { Hypothesis 4 }\end{array}$ \\
\hline Africa & 0.13 & No \\
\hline Asia Pacific & 0.76 & Yes \\
\hline Europe & 0.29 & Yes \\
\hline Latin America & 0.37 & Yes \\
\hline Middle East & 0.68 & Yes \\
\hline North America & 0.43 & Yes \\
\hline Russia \& Caspian & 0.24 & No \\
\hline
\end{tabular}




\section{APPENDIX 11}

Hypothesis IV - Demographic Breakdown - Summary and Hemisphere Structural Models for Perceived Organizational Support and Affective Reaction

Hypothesis 4, that employee perceived support is positively related to affective reactions is supported across all regions but the Africa and Russia \& Caspian regions. The Gulf of Mexico region was excluded due to its small sample size (4).

\section{Hemispherical Structural Models for Perceived Organizational Support and Affective Reaction}

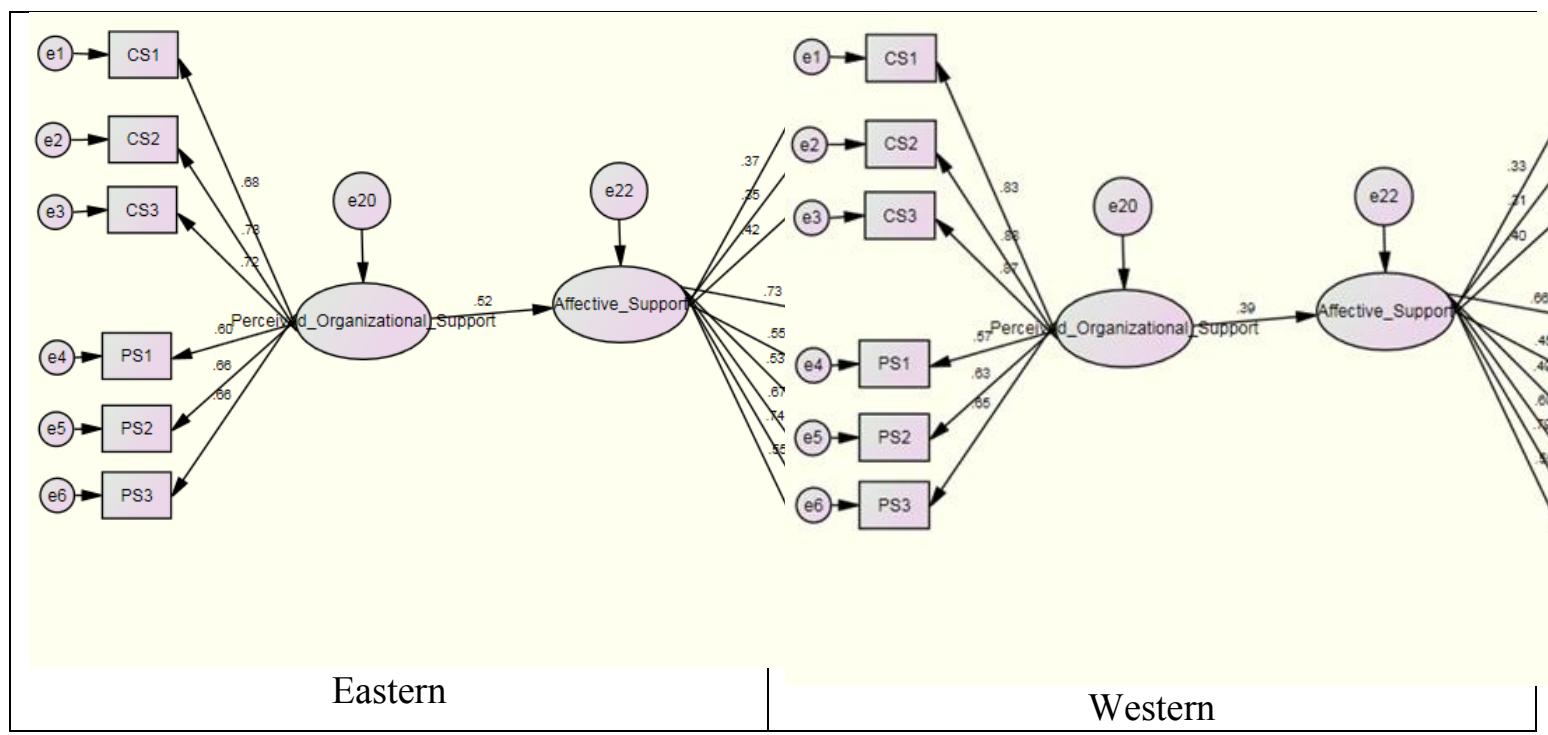

Summary of Model Fit Indices Perceived Organizational Support and Affective Reaction Hemisphere

\begin{tabular}{|l|c|c|}
\hline Model & NFI & CFI \\
\hline Eastern & 0.631 & 0.678 \\
\hline Western & 0.471 & 0.552 \\
\hline
\end{tabular}

Neither the NFI nor CFI are at sufficient level as to suggest a good model. This is to be expected as these are subsamples of a skewed distribution. 
Summary Table for Perceived Organizational Support and Affective Reaction Hemisphere

\begin{tabular}{|l|c|c|}
\hline & $r$ & $\begin{array}{c}\text { Support } \\
\text { Hypothesis 4 }\end{array}$ \\
\hline Eastern & 0.52 & Yes \\
\hline Western & 0.39 & Yes \\
\hline
\end{tabular}

Hypothesis 4, that employee perceived support is positively related to affective reactions is supported across both eastern and western hemispheres. 


\section{APPENDIX 12}

\section{Breakdown of Formal Mentoring Questions}

\section{Formal Mentoring Q1=Yes}

\begin{tabular}{|c|c|c|c|c|c|}
\hline \multirow{13}{*}{ Gender } & & Undetermined & Male & Female & Total \\
\hline & Africa & 2 & 31 & 5 & 38 \\
\hline & Asia Pacific & 1 & 35 & 10 & 46 \\
\hline & Europe & 1 & 9 & 6 & 16 \\
\hline & Latin America & 1 & 23 & 10 & 34 \\
\hline & Middle East & 1 & 24 & 2 & 27 \\
\hline & North America & 1 & 35 & 4 & 40 \\
\hline & Russia \& Caspian & 2 & 18 & 0 & 20 \\
\hline & Total & 9 & 175 & 37 & 221 \\
\hline & & Undetermined & Male & Female & Total \\
\hline & Eastern & 7 & 117 & 23 & 147 \\
\hline & Western & 2 & 58 & 14 & 74 \\
\hline & Total & 9 & 175 & 37 & 221 \\
\hline
\end{tabular}

\begin{tabular}{|c|c|c|c|c|c|c|c|c|c|}
\hline \multirow{9}{*}{$\begin{array}{c}\text { How } \\
\text { long } \\
\text { have } \\
\text { you } \\
\text { been } \\
\text { employ } \\
\text { ed with } \\
\text { Baker } \\
\text { Hughes } \\
?\end{array}$} & & $\begin{array}{r}\text { Undet } \\
\text { ermin } \\
\text { ed }\end{array}$ & $<6 \mathrm{mo}$ & $\begin{array}{l}6 \mathrm{mo}> \\
1 \mathrm{yr}\end{array}$ & $\begin{array}{l}1 \mathrm{yr}> \\
3 \mathrm{yrs}\end{array}$ & $\begin{array}{l}3 \mathrm{yrs}>5 \\
\mathrm{yrs}\end{array}$ & $\begin{array}{l}5 \mathrm{yrs}>8 \\
\mathrm{yrs}\end{array}$ & $\begin{array}{l}8 \mathrm{yrs}>1 \\
0 \mathrm{yrs}\end{array}$ & Total \\
\hline & Africa & 2 & 3 & 15 & 17 & 1 & 0 & 0 & 38 \\
\hline & $\begin{array}{l}\text { Asia } \\
\text { Pacific }\end{array}$ & 1 & 4 & 24 & 12 & 5 & 0 & 0 & 46 \\
\hline & Europe & 1 & 2 & 11 & 2 & 0 & 0 & 0 & 16 \\
\hline & $\begin{array}{l}\text { Latin } \\
\text { America }\end{array}$ & 1 & 1 & 12 & 15 & 4 & 0 & 1 & 34 \\
\hline & $\begin{array}{l}\text { Middle } \\
\text { East }\end{array}$ & 1 & 2 & 11 & 6 & 4 & 1 & 2 & 27 \\
\hline & $\begin{array}{l}\text { North } \\
\text { America }\end{array}$ & 1 & 12 & 18 & 8 & 0 & 1 & 0 & 40 \\
\hline & $\begin{array}{l}\text { Russia \& } \\
\text { Caspian }\end{array}$ & 1 & 2 & 10 & 5 & 2 & 0 & 0 & 20 \\
\hline & Total & 8 & 26 & 101 & 65 & 16 & 2 & 3 & 221 \\
\hline
\end{tabular}




\begin{tabular}{|c|c|c|c|c|c|c|c|c|c|}
\hline \multirow{4}{*}{$\begin{array}{l}\text { How } \\
\text { long } \\
\text { have } \\
\text { you } \\
\text { been } \\
\text { employ } \\
\text { ed with } \\
\text { Baker } \\
\text { Hughes } \\
?\end{array}$} & & $\begin{array}{r}\text { Undet } \\
\text { ermine } \\
d\end{array}$ & $<6 \mathrm{mo}$ & $\begin{array}{l}6 \mathrm{mo}> \\
1 \mathrm{yr}\end{array}$ & $\begin{array}{l}1 \mathrm{yr}> \\
3 \mathrm{yrs}\end{array}$ & $\begin{array}{l}3 \mathrm{yrs}>5 \\
\mathrm{yrs}\end{array}$ & $\begin{array}{l}5 \mathrm{yrs}>8 \\
\mathrm{yrs}\end{array}$ & $\begin{array}{l}8 \mathrm{yrs}>1 \\
0 \mathrm{yrs}\end{array}$ & Total \\
\hline & Eastern & 6 & 13 & 71 & 42 & 12 & 1 & 2 & 147 \\
\hline & Western & 2 & 13 & 30 & 23 & 4 & 1 & 1 & 74 \\
\hline & Total & 8 & 26 & 101 & 65 & 16 & 2 & 3 & 221 \\
\hline
\end{tabular}

\begin{tabular}{|c|c|c|c|c|c|c|c|}
\hline \multirow{13}{*}{$\begin{array}{c}\text { How } \\
\text { long } \\
\text { have you } \\
\text { been } \\
\text { employe } \\
\text { d at your } \\
\text { current } \\
\text { position? }\end{array}$} & & $\begin{array}{r}\text { Undeter } \\
\text { mined }\end{array}$ & $<6 \mathrm{mo}$ & $\begin{array}{l}6 \mathrm{mo}> \\
1 \mathrm{yr} \\
\end{array}$ & $\begin{array}{l}1 \mathrm{yr}> \\
3 \mathrm{yrs}\end{array}$ & $\begin{array}{l}3 y r s>5 y r \\
s\end{array}$ & Total \\
\hline & Africa & 3 & 6 & 14 & 15 & 0 & 38 \\
\hline & Asia Pacific & 2 & 5 & 23 & 13 & 3 & 46 \\
\hline & Europe & 1 & 3 & 11 & 1 & 0 & 16 \\
\hline & Latin America & 1 & 5 & 11 & 16 & 1 & 34 \\
\hline & Middle East & 1 & 2 & 11 & 8 & 5 & 27 \\
\hline & $\begin{array}{l}\text { North } \\
\text { America }\end{array}$ & 1 & 16 & 15 & 7 & 1 & 40 \\
\hline & $\begin{array}{l}\text { Russia \& } \\
\text { Caspian }\end{array}$ & 1 & 3 & 9 & 5 & 2 & 20 \\
\hline & Total & 10 & 40 & 94 & 65 & 12 & 221 \\
\hline & & $\begin{array}{r}\text { Undeter } \\
\text { mined }\end{array}$ & $<6 \mathrm{mo}$ & $\begin{array}{l}6 \mathrm{mo}> \\
1 \mathrm{yr} \\
\end{array}$ & $\begin{array}{l}1 \mathrm{yr}> \\
3 \mathrm{yrs}\end{array}$ & $\begin{array}{l}3 \mathrm{yrs}>5 \mathrm{yr} \\
\mathrm{s}\end{array}$ & Total \\
\hline & Eastern & 8 & 19 & 68 & 42 & 10 & 147 \\
\hline & Western & 2 & 21 & 26 & 23 & 2 & 74 \\
\hline & Total & 10 & 40 & 94 & 65 & 12 & 221 \\
\hline
\end{tabular}

Formal Mentoring Q1=Yes Q2=Yes

\begin{tabular}{|l|l|r|r|r|r|}
\hline \multirow{7}{*}{} & & Undetermined & Male & Female & Total \\
\cline { 2 - 6 } & Africa & 1 & 28 & 4 & 33 \\
\cline { 2 - 6 } & Asia Pacific & 1 & 25 & 9 & 35 \\
\cline { 2 - 6 } & Europe & 1 & 6 & 5 & 12 \\
\cline { 2 - 6 } & Latin America & 1 & 20 & 4 & 25 \\
\cline { 2 - 6 } & Middle East & 0 & 18 & 2 & 20 \\
\cline { 2 - 6 } & North America & 1 & 29 & 3 & 33 \\
\cline { 2 - 6 } & Russia \& Caspian & 2 & 16 & 0 & 18 \\
\cline { 2 - 6 } & Total & 7 & 142 & 27 & 176 \\
\hline
\end{tabular}




\begin{tabular}{|l|l|r|r|r|r|}
\hline \multirow{4}{*}{ Gender } & & Undetermined & Male & Female & \multicolumn{2}{|c|}{ Total } \\
\cline { 2 - 7 } & Eastern & 5 & 93 & 20 & 118 \\
\cline { 2 - 7 } & Western & 2 & 49 & 7 & 58 \\
\cline { 2 - 7 } & Total & 7 & 142 & 27 & 176 \\
\hline
\end{tabular}

\begin{tabular}{|c|c|c|c|c|c|c|c|c|}
\hline \multirow{13}{*}{$\begin{array}{c}\text { How } \\
\text { long } \\
\text { have you } \\
\text { been } \\
\text { employe } \\
\text { d with } \\
\text { Baker } \\
\text { Hughes? }\end{array}$} & & $\begin{array}{l}\text { Undeter } \\
\text { mined }\end{array}$ & $<6 \mathrm{mo}$ & $\begin{array}{l}6 \mathrm{mo}> \\
1 \mathrm{yr}\end{array}$ & $\begin{array}{l}1 \mathrm{yr}> \\
3 \mathrm{yrs}\end{array}$ & $\begin{array}{l}3 \mathrm{yrs}>5 \mathrm{y} \\
\text { rs }\end{array}$ & $\begin{array}{l}5 y r s>8 y \\
\text { rs }\end{array}$ & Total \\
\hline & Africa & 1 & 3 & 13 & 15 & 1 & 0 & 33 \\
\hline & $\begin{array}{l}\text { Asia } \\
\text { Pacific }\end{array}$ & 1 & 4 & 21 & 7 & 2 & 0 & 35 \\
\hline & Europe & 1 & 2 & 8 & 1 & 0 & 0 & 12 \\
\hline & $\begin{array}{l}\text { Latin } \\
\text { America }\end{array}$ & 1 & 1 & 8 & 12 & 2 & 1 & 25 \\
\hline & $\begin{array}{l}\text { Middle } \\
\text { East }\end{array}$ & 0 & 1 & 11 & 5 & 2 & 1 & 20 \\
\hline & $\begin{array}{l}\text { North } \\
\text { America }\end{array}$ & 1 & 11 & 16 & 5 & 0 & 0 & 33 \\
\hline & $\begin{array}{l}\text { Russia } \\
\& \\
\text { Caspian }\end{array}$ & 1 & 2 & 9 & 4 & 2 & 0 & 18 \\
\hline & Total & 6 & 24 & 86 & 49 & 9 & 2 & 176 \\
\hline & & $\begin{array}{l}\text { Undeter } \\
\text { mined }\end{array}$ & $<6 \mathrm{mo}$ & $\begin{array}{l}6 \mathrm{mo}> \\
1 \mathrm{yr}\end{array}$ & $\begin{array}{l}1 \mathrm{yr}> \\
3 \mathrm{yrs}\end{array}$ & $\begin{array}{l}3 y r s>5 y \\
\text { rs }\end{array}$ & $\begin{array}{l}5 y r s>8 y \\
\text { rs }\end{array}$ & Total \\
\hline & Eastern & 4 & 12 & 62 & 32 & 7 & 1 & 118 \\
\hline & Western & 2 & 12 & 24 & 17 & 2 & 1 & 58 \\
\hline & Total & 6 & 24 & 86 & 49 & 9 & 2 & 176 \\
\hline
\end{tabular}

\begin{tabular}{|c|c|c|c|c|c|c|c|}
\hline \multirow{7}{*}{$\begin{array}{c}\text { How } \\
\text { long } \\
\text { have you } \\
\text { been } \\
\text { employe } \\
\text { d at your } \\
\text { current } \\
\text { position? }\end{array}$} & & $\begin{array}{l}\text { Undeter } \\
\text { mined }\end{array}$ & $<6 \mathrm{mo}$ & $\begin{array}{l}6 \mathrm{mo}> \\
1 \mathrm{yr}\end{array}$ & $\begin{array}{l}1 \mathrm{yr}> \\
3 \mathrm{yrs}\end{array}$ & $\begin{array}{l}3 y r s>5 y r \\
s\end{array}$ & Total \\
\hline & Africa & 2 & 6 & 12 & 13 & 0 & 33 \\
\hline & Asia Pacific & 2 & 4 & 20 & 8 & 1 & 35 \\
\hline & Europe & 1 & 3 & 8 & 0 & 0 & 12 \\
\hline & Latin America & 1 & 3 & 8 & 13 & 0 & 25 \\
\hline & Middle East & 0 & 1 & 11 & 7 & 1 & 20 \\
\hline & North America & 1 & 15 & 13 & 4 & 0 & 33 \\
\hline
\end{tabular}




\begin{tabular}{|l|l|r|r|r|r|r|r|}
\hline $\begin{array}{l}\text { Russia \& } \\
\text { Caspian }\end{array}$ & 1 & 3 & 8 & 4 & 2 & 18 \\
\cline { 2 - 8 } & Total & 8 & 35 & 80 & 49 & 4 & 176 \\
\cline { 2 - 8 } & $\begin{array}{l}\text { Undeter } \\
\text { mined }\end{array}$ & $<6 \mathrm{mo}$ & $\begin{array}{l}\text { 6mo }> \\
1 \mathrm{yr}\end{array}$ & $\begin{array}{l}\text { 1yr }> \\
\text { 3yrs }\end{array}$ & $\begin{array}{l}3 \mathrm{yrs}>5 \mathrm{yr} \\
\text { s }\end{array}$ & Total \\
\cline { 2 - 8 } & Eastern & 6 & 17 & 59 & 32 & 4 & 118 \\
\cline { 2 - 8 } & Western & & 18 & 21 & 17 & 0 & 58 \\
\cline { 2 - 8 } & Total & & 35 & 80 & 49 & 4 & 176 \\
\hline
\end{tabular}

Formal Mentoring $Q 1=$ Yes $Q 2=$ Yes $Q 3=$ Yes

\begin{tabular}{|c|c|c|c|c|c|}
\hline \multirow{13}{*}{ Gender } & & Undetermined & Male & Female & Total \\
\hline & Africa & 1 & 17 & 2 & 20 \\
\hline & Asia Pacific & 1 & 19 & 8 & 28 \\
\hline & Europe & 0 & 1 & 2 & 3 \\
\hline & Latin America & 0 & 8 & 3 & 11 \\
\hline & Middle East & 0 & 12 & 2 & 14 \\
\hline & North America & 0 & 12 & 0 & 12 \\
\hline & Russia \& Caspian & 1 & 11 & 0 & 12 \\
\hline & Total & 3 & 80 & 17 & 100 \\
\hline & & Undetermined & Male & Female & Total \\
\hline & Eastern & 3 & 60 & 14 & 77 \\
\hline & Western & 0 & 20 & 3 & 23 \\
\hline & Total & 3 & 80 & 17 & 100 \\
\hline
\end{tabular}

\begin{tabular}{|c|c|c|c|c|c|c|c|c|}
\hline \multirow{8}{*}{$\begin{array}{c}\text { How } \\
\text { long } \\
\text { have you } \\
\text { been } \\
\text { employe } \\
\text { d with } \\
\text { Baker } \\
\text { Hughes? }\end{array}$} & & $\begin{array}{l}\text { Undete } \\
\text { rmined }\end{array}$ & $<6 \mathrm{mo}$ & $\begin{array}{l}6 \mathrm{mo}> \\
1 \mathrm{yr}\end{array}$ & $\begin{array}{l}1 \mathrm{yr}> \\
3 \mathrm{yrs}\end{array}$ & $\begin{array}{l}3 y r s> \\
5 y r s\end{array}$ & $\begin{array}{l}5 y r s> \\
8 y r s\end{array}$ & Total \\
\hline & Africa & 1 & 2 & 9 & 8 & 0 & 0 & 20 \\
\hline & Asia Pacific & 1 & 2 & 17 & 6 & 2 & 0 & 28 \\
\hline & Europe & 0 & 2 & 1 & 0 & 0 & 0 & 3 \\
\hline & $\begin{array}{l}\text { Latin } \\
\text { America }\end{array}$ & 0 & 0 & 3 & 6 & 2 & 0 & 11 \\
\hline & Middle East & 0 & 0 & 7 & 4 & 2 & 1 & 14 \\
\hline & $\begin{array}{l}\text { North } \\
\text { America }\end{array}$ & 0 & 3 & 7 & 2 & 0 & 0 & 12 \\
\hline & $\begin{array}{l}\text { Russia \& } \\
\text { Caspian }\end{array}$ & 1 & 0 & 7 & 3 & 1 & 0 & 12 \\
\hline
\end{tabular}




\begin{tabular}{|l|l|r|r|r|r|r|r|r|}
\hline & Total & 3 & 9 & 51 & 29 & 7 & 1 & 100 \\
\cline { 2 - 10 } & $\begin{array}{l}\text { Undete } \\
\text { rmined }\end{array}$ & $<6 \mathrm{mo}$ & $\begin{array}{l}6 \mathrm{mo}> \\
1 \mathrm{yr}\end{array}$ & $\begin{array}{l}1 \mathrm{yr}> \\
3 \mathrm{yrs}\end{array}$ & $\begin{array}{l}\text { 3yrs }>5 \\
\text { yrs }\end{array}$ & $\begin{array}{l}5 \mathrm{yrs}>8 \\
\text { yrs }\end{array}$ & Total \\
\cline { 2 - 9 } & Eastern & 3 & 6 & 41 & 21 & 5 & 1 & 77 \\
\cline { 2 - 9 } & Western & 0 & 3 & 10 & 8 & 2 & 0 & 23 \\
\cline { 2 - 9 } & Total & 3 & 9 & 51 & 29 & 7 & 1 & 100 \\
\hline
\end{tabular}

\begin{tabular}{|c|c|c|c|c|c|c|c|}
\hline \multirow{13}{*}{$\begin{array}{c}\text { How } \\
\text { long have } \\
\text { you been } \\
\text { employe } \\
\text { d at your } \\
\text { current } \\
\text { position? }\end{array}$} & & Undetermined & $<6 \mathrm{mo}$ & $\begin{array}{l}6 \mathrm{mo}> \\
1 \mathrm{yr}\end{array}$ & $\begin{array}{l}1 \mathrm{yr}> \\
3 \mathrm{yrs}\end{array}$ & $\begin{array}{l}3 y r s>5 y r \\
s\end{array}$ & Total \\
\hline & Africa & 1 & 4 & 8 & 7 & 0 & 20 \\
\hline & Asia Pacific & 1 & 2 & 17 & 7 & 1 & 28 \\
\hline & Europe & 0 & 2 & 1 & 0 & 0 & 3 \\
\hline & $\begin{array}{l}\text { Latin } \\
\text { America }\end{array}$ & 0 & 1 & 3 & 7 & 0 & 11 \\
\hline & Middle East & 0 & 0 & 7 & 6 & 1 & 14 \\
\hline & $\begin{array}{l}\text { North } \\
\text { America }\end{array}$ & 0 & 4 & 6 & 2 & 0 & 12 \\
\hline & $\begin{array}{l}\text { Russia \& } \\
\text { Caspian }\end{array}$ & 1 & 1 & 6 & 3 & 1 & 12 \\
\hline & Total & 3 & 14 & 48 & 32 & 3 & 100 \\
\hline & & Undetermined & $<6 \mathrm{mo}$ & $\begin{array}{l}6 \mathrm{mo}> \\
1 \mathrm{yr}\end{array}$ & $\begin{array}{l}1 \mathrm{yr}> \\
3 \mathrm{yrs}\end{array}$ & $\begin{array}{l}3 \mathrm{yrs}>5 \mathrm{yr} \\
\mathrm{s}\end{array}$ & Total \\
\hline & Eastern & 3 & 9 & 39 & 23 & 3 & 77 \\
\hline & Western & 0 & 5 & 9 & 9 & 0 & 23 \\
\hline & Total & 3 & 14 & 48 & 32 & 3 & 100 \\
\hline
\end{tabular}

Formal Mentoring Q1=Yes Q2=Yes Q3=Yes Q4=Yes

\begin{tabular}{|c|l|r|r|r|r|}
\hline & & Undetermined & Male & Female & \multicolumn{1}{|c|}{ Total } \\
\cline { 2 - 6 } & Africa & 1 & 6 & 1 & 8 \\
\cline { 2 - 6 } Gender & Asia Pacific & 1 & 10 & 2 & 13 \\
\cline { 2 - 7 } & Latin America & 0 & 2 & 2 & 4 \\
\cline { 2 - 7 } & Middle East & 0 & 6 & 0 & 6 \\
\cline { 2 - 7 } & North America & 0 & 2 & 0 & 2 \\
\cline { 2 - 7 } & Russia \& Caspian & 1 & 8 & 0 & 42 \\
\cline { 2 - 7 } & Total & 3 & 34 & 5 & 4 \\
\hline
\end{tabular}




\begin{tabular}{|l|r|r|r|r|} 
& Undetermined & Male & Female & Total \\
\hline Eastern & 3 & 30 & 3 & 36 \\
\hline Western & 0 & 4 & 2 & 6 \\
\hline Total & 3 & 34 & 5 & 42 \\
\hline
\end{tabular}

\begin{tabular}{|c|c|c|c|c|c|c|c|c|}
\hline \multirow{12}{*}{$\begin{array}{c}\text { How } \\
\text { long } \\
\text { have you } \\
\text { been } \\
\text { employe } \\
\text { d with } \\
\text { Baker } \\
\text { Hughes? }\end{array}$} & & Undetermined & $<6 \mathrm{mo}$ & $\begin{array}{l}6 \mathrm{mo}> \\
1 \mathrm{yr}\end{array}$ & $\begin{array}{l}1 \mathrm{yr}> \\
3 \mathrm{yrs}\end{array}$ & $\begin{array}{l}\text { 3yrs }> \\
5 y r s\end{array}$ & $\begin{array}{l}5 y r s> \\
8 y r s\end{array}$ & $\begin{array}{l}\text { Tota } \\
1\end{array}$ \\
\hline & Africa & 1 & 1 & 2 & 4 & 0 & 0 & 8 \\
\hline & $\begin{array}{l}\text { Asia } \\
\text { Pacific }\end{array}$ & 1 & 0 & 9 & 2 & 1 & 0 & 13 \\
\hline & $\begin{array}{l}\text { Latin } \\
\text { America }\end{array}$ & 0 & 0 & 0 & 3 & 1 & 0 & 4 \\
\hline & $\begin{array}{l}\text { Middle } \\
\text { East }\end{array}$ & 0 & 0 & 3 & 1 & 1 & 1 & 6 \\
\hline & $\begin{array}{l}\text { North } \\
\text { America }\end{array}$ & 0 & 0 & 2 & 0 & 0 & 0 & 2 \\
\hline & $\begin{array}{l}\text { Russia } \\
\& \\
\text { Caspian }\end{array}$ & 1 & 0 & 4 & 3 & 1 & 0 & 9 \\
\hline & Total & 3 & 1 & 20 & 13 & 4 & 1 & 42 \\
\hline & & Undetermined & $<6 \mathrm{mo}$ & $\begin{array}{l}6 \mathrm{mo}> \\
1 \mathrm{yr}\end{array}$ & $\begin{array}{l}1 \mathrm{yr}> \\
3 \mathrm{yrs}\end{array}$ & $\begin{array}{l}3 \mathrm{yrs}> \\
5 \mathrm{yrs}\end{array}$ & $\begin{array}{l}\text { 5yrs }> \\
8 y r s\end{array}$ & $\begin{array}{l}\text { Tota } \\
1\end{array}$ \\
\hline & Eastern & 3 & 1 & 18 & 10 & 3 & 1 & 36 \\
\hline & Western & 0 & 0 & 2 & 3 & 1 & 0 & 6 \\
\hline & & 3 & 1 & 20 & 13 & 4 & 1 & 42 \\
\hline
\end{tabular}

\begin{tabular}{|c|c|c|c|c|c|c|c|}
\hline \multirow{8}{*}{$\begin{array}{c}\text { How } \\
\text { long have } \\
\text { you been } \\
\text { employed } \\
\text { at your } \\
\text { current } \\
\text { position? }\end{array}$} & & Undetermined & $<6 \mathrm{mo}$ & $\begin{array}{l}6 \mathrm{mo}> \\
1 \mathrm{yr} \\
\end{array}$ & $\begin{array}{l}1 \mathrm{yr}> \\
3 \mathrm{yrs}\end{array}$ & $\begin{array}{l}3 \mathrm{yrs}> \\
5 \mathrm{yrs}\end{array}$ & Total \\
\hline & Africa & 1 & 1 & 2 & 4 & 0 & 8 \\
\hline & Asia Pacific & 1 & 0 & 9 & 2 & 1 & 13 \\
\hline & Latin America & 0 & 1 & 0 & 3 & 0 & 4 \\
\hline & Middle East & 0 & 0 & 4 & 1 & 1 & 6 \\
\hline & $\begin{array}{l}\text { North } \\
\text { America }\end{array}$ & 0 & 0 & 2 & 0 & 0 & 2 \\
\hline & $\begin{array}{l}\text { Russia \& } \\
\text { Caspian }\end{array}$ & 1 & 1 & 3 & 3 & 1 & 9 \\
\hline & & 3 & 3 & 20 & 13 & 3 & 42 \\
\hline
\end{tabular}




\begin{tabular}{|c|c|c|c|c|c|c|c|}
\hline \multirow{4}{*}{$\begin{array}{l}\text { How } \\
\text { long have } \\
\text { you been } \\
\text { employed } \\
\text { at your } \\
\text { current } \\
\text { position? }\end{array}$} & & Undetermined & $<6 \mathrm{mo}$ & $\begin{array}{l}6 \mathrm{mo}> \\
1 \mathrm{yr} \\
\end{array}$ & $\begin{array}{l}1 \mathrm{yr}> \\
3 \mathrm{yrs}\end{array}$ & $\begin{array}{l}3 \text { yrs }> \\
5 y r s\end{array}$ & Total \\
\hline & Eastern & 3 & 2 & 18 & 10 & 3 & 36 \\
\hline & Western & 0 & 1 & 2 & 3 & 0 & 6 \\
\hline & Total & 3 & 3 & 20 & 13 & 3 & 42 \\
\hline
\end{tabular}

Formal Mentoring $Q 1=$ Yes $Q 2=$ Yes $Q 3=$ Yes $Q 4=$ No

\begin{tabular}{|c|c|c|c|c|}
\hline \multirow{13}{*}{ Gender } & & Male & Female & Total \\
\hline & Africa & 11 & 1 & 12 \\
\hline & Asia Pacific & 9 & 6 & 15 \\
\hline & Europe & 1 & 2 & 3 \\
\hline & Latin America & 6 & 1 & 7 \\
\hline & Middle East & 6 & 2 & 8 \\
\hline & North America & 10 & 0 & 10 \\
\hline & Russia \& Caspian & 3 & 0 & 3 \\
\hline & Total & 46 & 12 & 58 \\
\hline & & Male & Female & Total \\
\hline & Eastern & 30 & 11 & 41 \\
\hline & Western & 16 & 1 & 17 \\
\hline & Total & 46 & 12 & 58 \\
\hline
\end{tabular}

\begin{tabular}{|c|c|c|c|c|c|c|}
\hline \multirow{9}{*}{$\begin{array}{l}\text { How long } \\
\text { have you } \\
\text { been } \\
\text { employed } \\
\text { with Baker } \\
\text { Hughes? }\end{array}$} & & $<6 \mathrm{mo}$ & $\begin{array}{l}6 \mathrm{mo}> \\
1 \mathrm{yr}\end{array}$ & $\begin{array}{l}1 \mathrm{yr}> \\
3 \mathrm{yrs}\end{array}$ & $\begin{array}{l}3 \mathrm{yrs}> \\
5 \mathrm{yrs}\end{array}$ & Total \\
\hline & Africa & 1 & 7 & 4 & 0 & 12 \\
\hline & Asia Pacific & 2 & 8 & 4 & 1 & 15 \\
\hline & Europe & 2 & 1 & 0 & 0 & 3 \\
\hline & Latin America & 0 & 3 & 3 & 1 & 7 \\
\hline & Middle East & 0 & 4 & 3 & 1 & 8 \\
\hline & North America & 3 & 5 & 2 & 0 & 10 \\
\hline & Russia \& Caspian & 0 & 3 & 0 & 0 & 3 \\
\hline & Total & 8 & 31 & 16 & 3 & 58 \\
\hline
\end{tabular}




\begin{tabular}{|c|c|c|c|c|c|c|}
\hline \multirow{4}{*}{$\begin{array}{l}\text { How long } \\
\text { have you } \\
\text { been } \\
\text { employed } \\
\text { with Baker } \\
\text { Hughes? }\end{array}$} & & $<6 \mathrm{mo}$ & $\begin{array}{l}6 \mathrm{mo}> \\
1 \mathrm{yr}\end{array}$ & $\begin{array}{l}1 \mathrm{yr}> \\
3 \mathrm{yrs}\end{array}$ & $\begin{array}{l}3 \text { yrs }> \\
5 y r s\end{array}$ & Total \\
\hline & Eastern & 5 & 23 & 11 & 2 & 41 \\
\hline & Western & 3 & 8 & 5 & 1 & 17 \\
\hline & Total & 8 & 31 & 16 & 3 & 58 \\
\hline
\end{tabular}

\begin{tabular}{|c|c|c|c|c|c|}
\hline \multirow{13}{*}{$\begin{array}{l}\text { How long } \\
\text { have you } \\
\text { been } \\
\text { employed at } \\
\text { your current } \\
\text { position? }\end{array}$} & & $<6 \mathrm{mo}$ & $6 \mathrm{mo}>1 \mathrm{yr}$ & $1 \mathrm{yr}>3 \mathrm{yrs}$ & Total \\
\hline & Africa & 3 & 6 & 3 & 12 \\
\hline & Asia Pacific & 2 & 8 & 5 & 15 \\
\hline & Europe & 2 & 1 & 0 & 3 \\
\hline & Latin America & 0 & 3 & 4 & 7 \\
\hline & Middle East & 0 & 3 & 5 & 8 \\
\hline & North America & 4 & 4 & 2 & 10 \\
\hline & Russia \& Caspian & 0 & 3 & 0 & 3 \\
\hline & Total & 11 & 28 & 19 & 58 \\
\hline & & $<6 \mathrm{mo}$ & $6 \mathrm{mo}>1 \mathrm{yr}$ & $1 \mathrm{yr}>3 \mathrm{yrs}$ & Total \\
\hline & Eastern & 7 & 21 & 13 & 41 \\
\hline & Western & 4 & 7 & 6 & 17 \\
\hline & Total & 11 & 28 & 19 & 58 \\
\hline
\end{tabular}

\section{Formal Mentoring $\mathbf{Q} 1=$ Yes $\mathbf{Q}=$ Yes $\mathbf{Q}=\mathbf{N o}$}

\begin{tabular}{|l|l|r|r|}
\hline & & Male & Total \\
\cline { 2 - 5 } & Asia Pacific & 1 & 1 \\
\cline { 2 - 5 } & Latin America & 3 & 3 \\
\cline { 2 - 5 } & Middle East & 2 & 2 \\
\cline { 2 - 5 } & North America & 3 & 3 \\
\cline { 2 - 5 } & Total & 9 & 9 \\
\cline { 2 - 5 } & & Male & Total \\
\cline { 2 - 5 } & Eastern & 3 & 6 \\
\cline { 2 - 5 } & Western & 6 & 9 \\
\cline { 2 - 5 } & Total & 9 & \\
\hline
\end{tabular}




\begin{tabular}{|c|c|c|c|c|c|}
\hline \multirow{10}{*}{$\begin{array}{c}\text { How long } \\
\text { have you } \\
\text { been } \\
\text { employed } \\
\text { with Baker } \\
\text { Hughes? }\end{array}$} & & $<6 \mathrm{mo}$ & $6 \mathrm{mo}>1 \mathrm{yr}$ & $1 \mathrm{yr}>3 \mathrm{yrs}$ & Total \\
\hline & Asia Pacific & 0 & 0 & 1 & 1 \\
\hline & Latin America & 1 & 0 & 2 & 3 \\
\hline & Middle East & 1 & 1 & 0 & 2 \\
\hline & North America & 0 & 2 & 1 & 3 \\
\hline & Total & 2 & 3 & 4 & 9 \\
\hline & & $<6 \mathrm{mo}$ & $6 \mathrm{mo}>1 \mathrm{yr}$ & $1 \mathrm{yr}>3 \mathrm{yrs}$ & Total \\
\hline & Eastern & 1 & 1 & 1 & 3 \\
\hline & Western & 1 & 2 & 3 & 6 \\
\hline & Total & 2 & 3 & 4 & 9 \\
\hline
\end{tabular}

\begin{tabular}{|c|c|c|c|c|c|}
\hline \multirow{10}{*}{$\begin{array}{c}\text { How long } \\
\text { have you } \\
\text { been } \\
\text { employed at } \\
\text { your current } \\
\text { position? }\end{array}$} & & $<6 \mathrm{mo}$ & $6 \mathrm{mo}>1 \mathrm{yr}$ & $1 \mathrm{yr}>3 \mathrm{yrs}$ & Total \\
\hline & Asia Pacific & 0 & 0 & 1 & 1 \\
\hline & Latin America & 2 & 0 & 1 & 3 \\
\hline & Middle East & 1 & 1 & 0 & 2 \\
\hline & North America & 1 & 1 & 1 & 3 \\
\hline & Total & 4 & 2 & 3 & 9 \\
\hline & & $<6 \mathrm{mo}$ & $6 \mathrm{mo}>1 \mathrm{yr}$ & $1 \mathrm{yr}>3 \mathrm{yrs}$ & Total \\
\hline & Eastern & 1 & 1 & 1 & 3 \\
\hline & Western & 3 & 1 & 2 & 6 \\
\hline & Total & 4 & 2 & 3 & 9 \\
\hline
\end{tabular}

Formal Mentoring $Q 1=$ Yes $Q 2=$ Yes $Q 3=$ No $Q 4=$ Yes

\begin{tabular}{|l|l|r|r|}
\hline & & Male & Total \\
\cline { 2 - 5 } & Latin America & 1 & 1 \\
\cline { 2 - 5 } & Middle East & 2 & 2 \\
\cline { 2 - 5 } & North America & 1 & 1 \\
\cline { 2 - 5 } & Total & 4 & 4 \\
\cline { 2 - 5 } & & Male & Total \\
\cline { 2 - 5 } & Eastern & 2 & 2 \\
\cline { 2 - 5 } & Western & 2 & 4 \\
\cline { 2 - 5 } & Total & 4 & 2 \\
\hline
\end{tabular}




\begin{tabular}{|c|c|c|c|c|c|}
\hline \multirow{9}{*}{$\begin{array}{c}\text { How long } \\
\text { have you } \\
\text { been } \\
\text { employed } \\
\text { with Baker } \\
\text { Hughes? }\end{array}$} & & $<6 \mathrm{mo}$ & $6 \mathrm{mo}>1 \mathrm{yr}$ & $1 \mathrm{yr}>3 \mathrm{yrs}$ & Total \\
\hline & Latin America & 0 & 0 & 1 & 1 \\
\hline & Middle East & 1 & 1 & 0 & 2 \\
\hline & North America & 0 & 0 & 1 & 1 \\
\hline & Total & 1 & 1 & 2 & 2 \\
\hline & & $<6 \mathrm{mo}$ & $6 \mathrm{mo}>1 \mathrm{yr}$ & $1 \mathrm{yr}>3 \mathrm{yrs}$ & Total \\
\hline & Eastern & 1 & 1 & 0 & 2 \\
\hline & Western & 0 & 0 & 2 & 2 \\
\hline & Total & 1 & 1 & 2 & 2 \\
\hline
\end{tabular}

\begin{tabular}{|c|c|c|c|c|c|}
\hline \multirow{9}{*}{$\begin{array}{l}\text { How long } \\
\text { have you } \\
\text { been } \\
\text { employed at } \\
\text { your current } \\
\text { position? }\end{array}$} & & $<6 \mathrm{mo}$ & $6 \mathrm{mo}>1 \mathrm{yr}$ & $1 \mathrm{yr}>3 \mathrm{yrs}$ & Total \\
\hline & Latin America & 1 & 0 & 0 & 1 \\
\hline & Middle East & 1 & 1 & 0 & 2 \\
\hline & North America & 0 & 0 & 1 & 1 \\
\hline & Total & 2 & 1 & 1 & 4 \\
\hline & & $<6 \mathrm{mo}$ & $6 \mathrm{mo}>1 \mathrm{yr}$ & $1 \mathrm{yr}>3 \mathrm{yrs}$ & Total \\
\hline & Eastern & 1 & 1 & 0 & 2 \\
\hline & Western & 1 & 0 & 1 & 2 \\
\hline & Total & 2 & 1 & 1 & 4 \\
\hline
\end{tabular}

Formal Mentoring $Q 1=$ Yes $Q 2=$ Yes $Q 3=$ No $Q 4=$ No

\begin{tabular}{|c|c|c|c|}
\hline \multirow{9}{*}{ Gender } & & Male & Total \\
\hline & Asia Pacific & 1 & 1 \\
\hline & Latin America & 2 & 2 \\
\hline & North America & 2 & 2 \\
\hline & Total & 5 & 5 \\
\hline & & Male & Total \\
\hline & Eastern & 1 & 1 \\
\hline & Western & 4 & 4 \\
\hline & Total & 5 & 5 \\
\hline
\end{tabular}




\begin{tabular}{|c|c|c|c|c|c|}
\hline \multirow{9}{*}{$\begin{array}{l}\text { How long } \\
\text { have you } \\
\text { been } \\
\text { employed } \\
\text { with Baker } \\
\text { Hughes? }\end{array}$} & & $<6 \mathrm{mo}$ & $6 \mathrm{mo}>1 \mathrm{yr}$ & $1 \mathrm{yr}>3 \mathrm{yrs}$ & $\begin{array}{l}\text { Tota } \\
1\end{array}$ \\
\hline & Asia Pacific & 0 & 0 & 1 & 1 \\
\hline & Latin America & 1 & 0 & 1 & 2 \\
\hline & North America & 0 & 2 & 0 & 2 \\
\hline & Total & 1 & 2 & 2 & 5 \\
\hline & & $<6 \mathrm{mo}$ & $6 \mathrm{mo}>1 \mathrm{yr}$ & $1 \mathrm{yr}>3 \mathrm{yrs}$ & $\begin{array}{l}\text { Tota } \\
1\end{array}$ \\
\hline & Eastern & 0 & 0 & 1 & 1 \\
\hline & Western & 1 & 2 & 1 & 4 \\
\hline & Total & 1 & 2 & 2 & 5 \\
\hline
\end{tabular}

\begin{tabular}{|c|c|c|c|c|c|}
\hline \multirow{9}{*}{$\begin{array}{l}\text { How long } \\
\text { have you } \\
\text { been } \\
\text { employed at } \\
\text { your current } \\
\text { position? }\end{array}$} & & $<6 \mathrm{mo}$ & $6 \mathrm{mo}>1 \mathrm{yr}$ & $1 \mathrm{yr}>3 \mathrm{yrs}$ & Total \\
\hline & Asia Pacific & 0 & 0 & 1 & 1 \\
\hline & Latin America & 1 & 0 & 1 & 2 \\
\hline & North America & 1 & 1 & 0 & 2 \\
\hline & Total & 2 & 1 & 2 & 5 \\
\hline & & $<6 \mathrm{mo}$ & $6 \mathrm{mo}>1 \mathrm{yr}$ & $1 \mathrm{yr}>3 \mathrm{yrs}$ & Total \\
\hline & Eastern & 0 & 0 & 1 & 1 \\
\hline & Western & 2 & 1 & 1 & 4 \\
\hline & Total & 2 & 1 & 2 & 5 \\
\hline
\end{tabular}

Formal Mentoring $Q 1=$ Yes $Q 2=$ No

\begin{tabular}{|c|l|r|r|r|r|}
\hline & Undetermined & Male & Female & Total \\
\cline { 2 - 6 } & Africa & 1 & 3 & 1 & 5 \\
\cline { 2 - 6 } & Asia Pacific & 0 & 10 & 1 & 11 \\
\cline { 2 - 6 } Gender & Europe & 0 & 3 & 1 & 4 \\
\cline { 2 - 6 } & Latin America & 0 & 3 & 6 & 9 \\
\cline { 2 - 6 } & Middle East & 1 & 6 & 0 & 7 \\
\cline { 2 - 6 } & North America & 0 & 5 & 1 & 6 \\
\cline { 2 - 6 } & Russia \& Caspian & 0 & 2 & 0 & 2 \\
\cline { 2 - 6 } & Total & 2 & 32 & 10 & 44 \\
\hline
\end{tabular}




\begin{tabular}{|c|l|r|r|r|r|}
\hline & & Undetermined & \multicolumn{1}{|c|}{ Male } & Female & \multicolumn{1}{|c|}{ Total } \\
\cline { 2 - 7 } Gender & Eastern & 2 & 24 & 3 & 29 \\
\cline { 2 - 7 } & Western & 0 & 8 & 7 & 15 \\
\cline { 2 - 7 } & Total & 2 & 32 & 10 & 44 \\
\hline
\end{tabular}

\begin{tabular}{|c|c|c|c|c|c|c|c|c|c|}
\hline \multirow{13}{*}{$\begin{array}{c} \\
\\
\\
\text { How } \\
\text { long } \\
\text { have } \\
\text { you } \\
\text { been } \\
\text { employe } \\
\text { d with } \\
\text { Baker } \\
\text { Hughes } \\
\text { ? }\end{array}$} & \multicolumn{2}{|r|}{ Undetermined } & $\begin{array}{l}<6 \\
\text { mo }\end{array}$ & $\begin{array}{l}6 \mathrm{mo}> \\
1 \mathrm{yr}\end{array}$ & $\begin{array}{l}1 \mathrm{yr}> \\
3 \mathrm{yrs}\end{array}$ & $\begin{array}{l}\text { 3yrs }> \\
5 \mathrm{yrs}\end{array}$ & $\begin{array}{l}5 y r s> \\
8 y r s\end{array}$ & $\begin{array}{l}8 \mathrm{yrs}> \\
10 \mathrm{yrs}\end{array}$ & Total \\
\hline & Africa & 1 & 0 & 2 & 2 & 0 & 0 & 0 & 5 \\
\hline & $\begin{array}{l}\text { Asia } \\
\text { Pacific }\end{array}$ & 0 & 0 & 3 & 5 & 3 & 0 & 0 & 11 \\
\hline & Europe & 0 & 0 & 3 & 1 & 0 & 0 & 0 & 4 \\
\hline & $\begin{array}{l}\text { Latin } \\
\text { America }\end{array}$ & 0 & 0 & 4 & 3 & 2 & 0 & 0 & 9 \\
\hline & $\begin{array}{l}\text { Middle } \\
\text { East }\end{array}$ & 1 & 1 & 0 & 1 & 2 & 1 & 1 & 7 \\
\hline & $\begin{array}{l}\text { North } \\
\text { America }\end{array}$ & 0 & 1 & 2 & 2 & 0 & 1 & 0 & 6 \\
\hline & $\begin{array}{l}\text { Russia } \\
\& \\
\text { Caspian }\end{array}$ & 0 & 0 & 1 & 1 & 0 & 0 & 0 & 2 \\
\hline & Total & 2 & 2 & 15 & 15 & 7 & 2 & 1 & 44 \\
\hline & & Undetermined & $\begin{array}{l}<6 \\
\text { mo }\end{array}$ & $\begin{array}{l}6 \mathrm{mo}> \\
1 \mathrm{yr}\end{array}$ & $\begin{array}{l}1 \mathrm{yr}> \\
3 \mathrm{yrs}\end{array}$ & $\begin{array}{l}3 \mathrm{yrs}> \\
5 \mathrm{yrs}\end{array}$ & $\begin{array}{l}5 \mathrm{yrs}> \\
8 \mathrm{yrs} \\
\end{array}$ & $\begin{array}{l}8 \mathrm{yrs}> \\
10 \mathrm{yrs}\end{array}$ & Total \\
\hline & Eastern & 2 & 1 & 9 & 10 & 5 & 1 & 1 & 29 \\
\hline & Western & 0 & 1 & 6 & 5 & 2 & 1 & 0 & 15 \\
\hline & Total & 2 & 2 & 15 & 15 & 7 & 2 & 1 & 44 \\
\hline
\end{tabular}

\begin{tabular}{|c|c|c|c|c|c|c|c|}
\hline \multirow{9}{*}{$\begin{array}{c}\text { How } \\
\text { long have } \\
\text { you been } \\
\text { employe } \\
\text { d at your } \\
\text { current } \\
\text { position? }\end{array}$} & \multicolumn{2}{|c|}{ Undetermined } & $<6 \mathrm{mo}$ & $\begin{array}{l}6 \mathrm{mo}> \\
1 \mathrm{yr} \\
\end{array}$ & $\begin{array}{l}1 \mathrm{yr}> \\
3 \mathrm{yrs}\end{array}$ & $\begin{array}{l}3 y r s> \\
5 y r s\end{array}$ & Total \\
\hline & Africa & 1 & 0 & 2 & 2 & 0 & 5 \\
\hline & Asia Pacific & 0 & 1 & 3 & 5 & 2 & 11 \\
\hline & Europe & 0 & 0 & 3 & 1 & 0 & 4 \\
\hline & Latin America & 0 & 2 & 3 & 3 & 1 & 9 \\
\hline & Middle East & 1 & 1 & 0 & 1 & 4 & 7 \\
\hline & North America & 0 & 1 & 2 & 2 & 1 & 6 \\
\hline & $\begin{array}{l}\text { Russia \& } \\
\text { Caspian }\end{array}$ & 0 & 0 & 1 & 1 & 0 & 2 \\
\hline & Total & 2 & 5 & 14 & 15 & 8 & 44 \\
\hline
\end{tabular}




\begin{tabular}{|c|c|c|c|c|c|c|c|}
\hline \multirow{4}{*}{$\begin{array}{l}\text { How } \\
\text { long have } \\
\text { you been } \\
\text { employe } \\
\text { d at your } \\
\text { current } \\
\text { position? }\end{array}$} & \multicolumn{2}{|r|}{ Undetermined } & \multirow{2}{*}{$\begin{array}{r}<6 \mathrm{mo} \\
2 \\
\end{array}$} & \multirow{2}{*}{$\begin{array}{l}6 \mathrm{mo}> \\
1 \mathrm{yr} \\
9\end{array}$} & \multirow{2}{*}{$\begin{array}{l}\begin{array}{l}1 \mathrm{yr}> \\
3 y r s\end{array} \\
10\end{array}$} & \multirow{2}{*}{$\begin{array}{l}\begin{array}{l}3 y r s> \\
5 y r s\end{array} \\
6\end{array}$} & \multirow{2}{*}{$\begin{array}{r}\text { Total } \\
29 \\
\end{array}$} \\
\hline & Eastern & 2 & & & & & \\
\hline & Western & 0 & 3 & 5 & 5 & 2 & 15 \\
\hline & Total & 2 & 5 & 14 & 15 & 8 & 44 \\
\hline
\end{tabular}

Formal Mentoring $Q 1=$ Yes $Q 2=$ No $Q 3=$ Yes

\begin{tabular}{|c|c|c|c|c|c|}
\hline \multirow{13}{*}{ Gender } & & Undetermined & Male & Female & Total \\
\hline & Africa & 1 & 2 & 1 & 4 \\
\hline & Asia Pacific & 0 & 6 & 0 & 6 \\
\hline & Europe & 0 & 1 & 1 & 2 \\
\hline & Latin America & 0 & 1 & 2 & 3 \\
\hline & Middle East & 0 & 3 & 0 & 3 \\
\hline & North America & 0 & 1 & 0 & 1 \\
\hline & Russia \& Caspian & 0 & 1 & 0 & 1 \\
\hline & Total & 1 & 15 & 4 & 20 \\
\hline & & Undetermined & Male & Female & Total \\
\hline & Eastern & 1 & 13 & 2 & 16 \\
\hline & Western & 0 & 2 & 2 & 4 \\
\hline & Total & 1 & 15 & 4 & 20 \\
\hline
\end{tabular}

\begin{tabular}{|c|c|c|c|c|c|c|c|c|}
\hline \multirow{7}{*}{$\begin{array}{c}\text { How } \\
\text { long have } \\
\text { you been } \\
\text { employe } \\
\text { d with } \\
\text { Baker } \\
\text { Hughes? }\end{array}$} & \multicolumn{2}{|c|}{ Undetermined } & $<6 \mathrm{mo}$ & $\begin{array}{l}6 \mathrm{mo} \\
>1 \mathrm{yr}\end{array}$ & $\begin{array}{l}1 \mathrm{yr}> \\
3 \mathrm{yrs}\end{array}$ & $\begin{array}{l}3 \mathrm{yrs}> \\
5 \mathrm{yrs}\end{array}$ & $\begin{array}{l}5 y r s> \\
8 y r s\end{array}$ & Total \\
\hline & Africa & 1 & 1 & 2 & 0 & 0 & 0 & 4 \\
\hline & $\begin{array}{l}\text { Asia } \\
\text { Pacific }\end{array}$ & 0 & 2 & 4 & 0 & 0 & 0 & 6 \\
\hline & Europe & 0 & 2 & 0 & 0 & 0 & 0 & 2 \\
\hline & $\begin{array}{l}\text { Latin } \\
\text { America }\end{array}$ & 0 & 0 & 2 & 1 & 0 & 0 & 3 \\
\hline & $\begin{array}{l}\text { Middle } \\
\text { East }\end{array}$ & 0 & 0 & 0 & 1 & 1 & 1 & 3 \\
\hline & $\begin{array}{l}\text { North } \\
\text { America }\end{array}$ & 0 & 0 & 0 & 0 & 1 & 0 & 1 \\
\hline
\end{tabular}




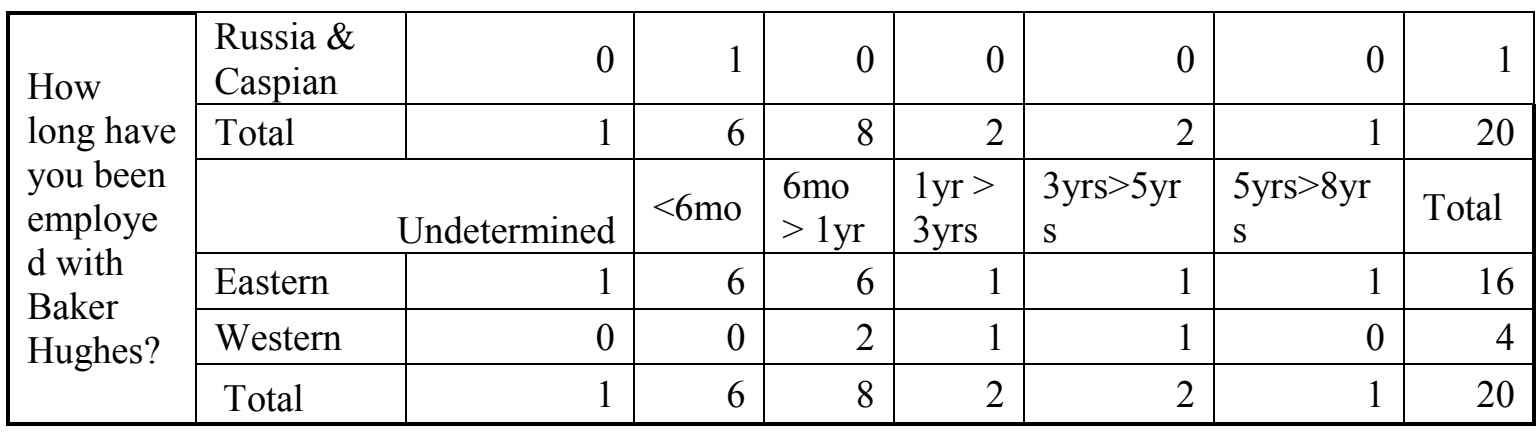

\begin{tabular}{|c|c|c|c|c|c|c|}
\hline \multirow{13}{*}{$\begin{array}{l}\text { How long } \\
\text { have you } \\
\text { been } \\
\text { employed } \\
\text { at your } \\
\text { current } \\
\text { position? }\end{array}$} & & Undetermined & $<6 \mathrm{mo}$ & $\begin{array}{l}6 \mathrm{mo}> \\
1 \mathrm{yr}\end{array}$ & $\begin{array}{l}1 \mathrm{yr}> \\
3 \mathrm{yrs}\end{array}$ & Total \\
\hline & Africa & 1 & 1 & 2 & 0 & 4 \\
\hline & Asia Pacific & 0 & 2 & 4 & 0 & 6 \\
\hline & Europe & 0 & 2 & 0 & 0 & 2 \\
\hline & Latin America & 0 & 0 & 2 & 1 & 3 \\
\hline & Middle East & 0 & 0 & 0 & 3 & 3 \\
\hline & North America & 0 & 0 & 0 & 1 & 1 \\
\hline & $\begin{array}{l}\text { Russia \& } \\
\text { Caspian }\end{array}$ & 0 & 1 & 0 & 0 & 1 \\
\hline & Total & 1 & 6 & 8 & 5 & 20 \\
\hline & & Undetermined & $<6 \mathrm{mo}$ & $\begin{array}{l}6 \mathrm{mo}> \\
1 \mathrm{yr}\end{array}$ & $\begin{array}{l}1 \mathrm{yr}> \\
3 \mathrm{yrs}\end{array}$ & Total \\
\hline & Eastern & 1 & 6 & 6 & 3 & 16 \\
\hline & Western & 0 & 0 & 2 & 2 & 4 \\
\hline & Total & 1 & 6 & 8 & 5 & 20 \\
\hline
\end{tabular}

Formal Mentoring Q1=Yes Q2=No Q3=Yes Q4=Yes

\begin{tabular}{|c|c|c|c|c|}
\hline \multirow{7}{*}{ Gender } & & Male & Female & Total \\
\hline & Africa & 0 & 1 & 1 \\
\hline & Asia Pacific & 4 & 0 & 4 \\
\hline & Europe & 1 & 1 & 2 \\
\hline & Latin America & 1 & 1 & 2 \\
\hline & Middle East & 2 & 0 & 2 \\
\hline & Total & 8 & 3 & 11 \\
\hline
\end{tabular}




\begin{tabular}{|l|l|r|r|r|}
\hline & & Male & Female & Total \\
\cline { 2 - 6 } Gender & Eastern & 7 & 2 & 9 \\
\cline { 2 - 6 } & Western & 1 & 1 & 2 \\
\cline { 2 - 6 } & Total & 8 & 3 & 11 \\
\hline
\end{tabular}

\begin{tabular}{|c|c|c|c|c|c|c|c|}
\hline \multirow{11}{*}{$\begin{array}{l}\text { How long } \\
\text { have you } \\
\text { been } \\
\text { employed } \\
\text { with } \\
\text { Baker } \\
\text { Hughes? }\end{array}$} & & $<6$ mo & $\begin{array}{l}6 \mathrm{mo}> \\
1 \mathrm{yr}\end{array}$ & $\begin{array}{l}1 \mathrm{yr}> \\
3 \mathrm{yrs}\end{array}$ & $\begin{array}{l}3 \mathrm{yrs}> \\
5 \mathrm{yrs}\end{array}$ & $\begin{array}{l}5 \mathrm{yrs}> \\
8 \mathrm{yrs}\end{array}$ & Total \\
\hline & Africa & 0 & 1 & 0 & 0 & 0 & 1 \\
\hline & Asia Pacific & 1 & 3 & 0 & 0 & 0 & 4 \\
\hline & Europe & 2 & 0 & 0 & 0 & 0 & 2 \\
\hline & Latin America & 0 & 1 & 1 & 0 & 0 & 2 \\
\hline & Middle East & 0 & 0 & 0 & 1 & 1 & 2 \\
\hline & Total & 3 & 5 & 1 & 1 & 1 & 11 \\
\hline & & $<6 \mathrm{mo}$ & $\begin{array}{l}6 \mathrm{mo}> \\
1 \mathrm{yr}\end{array}$ & $\begin{array}{l}1 \mathrm{yr}> \\
3 \mathrm{yrs}\end{array}$ & $\begin{array}{l}3 \mathrm{yrs}> \\
5 \mathrm{yrs}\end{array}$ & $\begin{array}{l}5 y r s> \\
8 y r s\end{array}$ & Total \\
\hline & Eastern & 3 & 4 & 0 & 1 & 1 & 9 \\
\hline & Western & 0 & 1 & 1 & 0 & 0 & 2 \\
\hline & Total & 3 & 5 & 1 & 1 & 1 & 11 \\
\hline
\end{tabular}

\begin{tabular}{|c|c|c|c|c|c|}
\hline \multirow{11}{*}{$\begin{array}{l}\text { How long } \\
\text { have you } \\
\text { been } \\
\text { employed at } \\
\text { your current } \\
\text { position? }\end{array}$} & & $<6 \mathrm{mo}$ & $6 \mathrm{mo}>1 \mathrm{yr}$ & $1 \mathrm{yr}>3 \mathrm{yrs}$ & Total \\
\hline & Africa & 0 & 1 & 0 & 1 \\
\hline & Asia Pacific & 1 & 3 & 0 & 4 \\
\hline & Europe & 2 & 0 & 0 & 2 \\
\hline & Latin America & 0 & 1 & 1 & 2 \\
\hline & Middle East & 0 & 0 & 2 & 2 \\
\hline & Total & 3 & 5 & 3 & 11 \\
\hline & & $<6 \mathrm{mo}$ & $6 \mathrm{mo}>1 \mathrm{yr}$ & $1 \mathrm{yr}>3 \mathrm{yrs}$ & Total \\
\hline & Eastern & 3 & 4 & 2 & 9 \\
\hline & Western & 0 & 1 & 1 & 2 \\
\hline & Total & 3 & 5 & 3 & 11 \\
\hline
\end{tabular}


Formal Mentoring $Q 1=$ Yes $Q 2=$ No $Q 3=$ Yes $Q 4=$ No

\begin{tabular}{|c|c|c|c|c|c|}
\hline \multirow{12}{*}{ Gender } & & Undetermined & Male & Female & Total \\
\hline & Africa & 1 & 2 & 0 & 3 \\
\hline & Asia Pacific & 0 & 2 & 0 & 2 \\
\hline & Latin America & 0 & 0 & 1 & 1 \\
\hline & Middle East & 0 & 1 & 0 & 1 \\
\hline & North America & 0 & 1 & 0 & 1 \\
\hline & Russia \& Caspian & 0 & 1 & 0 & 1 \\
\hline & Total & 1 & 7 & 1 & 9 \\
\hline & & Undetermined & Male & Female & Total \\
\hline & Eastern & 1 & 6 & 0 & 7 \\
\hline & Western & 0 & 1 & 1 & 2 \\
\hline & Total & 1 & 7 & 1 & 9 \\
\hline
\end{tabular}

\begin{tabular}{|c|c|c|c|c|c|c|c|}
\hline \multirow{12}{*}{$\begin{array}{c}\text { How } \\
\text { long have } \\
\text { you been } \\
\text { employe } \\
\text { d with } \\
\text { Baker } \\
\text { Hughes? }\end{array}$} & & Undetermined & $<6 \mathrm{mo}$ & $\begin{array}{l}6 \mathrm{mo}> \\
1 \mathrm{yr} \\
\end{array}$ & $\begin{array}{l}1 \mathrm{yr}> \\
3 \mathrm{yrs}\end{array}$ & $\begin{array}{l}3 y r s> \\
5 y r s\end{array}$ & Total \\
\hline & Africa & 1 & 1 & 1 & 0 & 0 & 3 \\
\hline & Asia Pacific & 0 & 1 & 1 & 0 & 0 & 2 \\
\hline & Latin America & 0 & 0 & 1 & 0 & 0 & 1 \\
\hline & Middle East & 0 & 0 & 0 & 1 & 0 & 1 \\
\hline & North America & 0 & 0 & 0 & 0 & 1 & 1 \\
\hline & $\begin{array}{l}\text { Russia \& } \\
\text { Caspian }\end{array}$ & 0 & 1 & 0 & 0 & 0 & 1 \\
\hline & Total & 1 & 3 & 3 & 1 & 1 & 9 \\
\hline & & Undetermined & $<6 \mathrm{mo}$ & $\begin{array}{l}6 \mathrm{mo}> \\
1 \mathrm{yr}\end{array}$ & $\begin{array}{l}1 \mathrm{yr}> \\
3 \mathrm{yrs}\end{array}$ & $\begin{array}{l}3 y r s> \\
5 y r s\end{array}$ & Total \\
\hline & Eastern & 1 & 3 & 2 & 1 & 0 & 7 \\
\hline & Western & 0 & 0 & 1 & 0 & 1 & 2 \\
\hline & Total & 1 & 3 & 3 & 1 & 1 & 9 \\
\hline
\end{tabular}




\begin{tabular}{|c|c|c|c|c|c|c|}
\hline \multirow{12}{*}{$\begin{array}{l}\text { How long } \\
\text { have you } \\
\text { been } \\
\text { employed } \\
\text { at your } \\
\text { current } \\
\text { position? }\end{array}$} & & Undetermined & $<6 \mathrm{mo}$ & $\begin{array}{l}6 \mathrm{mo}> \\
1 \mathrm{yr} \\
\end{array}$ & $\begin{array}{l}1 \mathrm{yr}> \\
3 \mathrm{yrs}\end{array}$ & Total \\
\hline & Africa & 1 & 1 & 1 & 0 & 3 \\
\hline & Asia Pacific & 0 & 1 & 1 & 0 & 2 \\
\hline & Latin America & 0 & 0 & 1 & 0 & 1 \\
\hline & Middle East & 0 & 0 & 0 & 1 & 1 \\
\hline & North America & 0 & 0 & 0 & 1 & 1 \\
\hline & $\begin{array}{l}\text { Russia \& } \\
\text { Caspian } \\
\end{array}$ & 0 & 1 & 0 & 0 & 1 \\
\hline & Total & 1 & 3 & 3 & 2 & 9 \\
\hline & & Undetermined & $<6 \mathrm{mo}$ & $\begin{array}{l}6 \mathrm{mo}> \\
1 \mathrm{yr}\end{array}$ & $\begin{array}{l}1 \mathrm{yr}> \\
3 \mathrm{yrs}\end{array}$ & Total \\
\hline & Eastern & 1 & 3 & 2 & 1 & 7 \\
\hline & Western & 0 & 0 & 1 & 1 & 2 \\
\hline & Total & 1 & 3 & 3 & 2 & 9 \\
\hline
\end{tabular}

Formal Mentoring $\mathbf{Q 1}=$ Yes $\mathbf{Q}=\mathbf{N o} \mathbf{Q 3}=\mathbf{N o}$

\begin{tabular}{|c|c|c|c|c|c|}
\hline \multirow{9}{*}{ Gender } & & Undetermined & Male & Female & Total \\
\hline & Latin America & 0 & 0 & 1 & 1 \\
\hline & Middle East & 1 & 0 & 0 & 1 \\
\hline & North America & 0 & 2 & 1 & 3 \\
\hline & Total & 1 & 2 & 2 & 5 \\
\hline & & Undetermined & Male & Female & Total \\
\hline & Eastern & 1 & 0 & 0 & 1 \\
\hline & Western & 0 & 2 & 2 & 4 \\
\hline & Total & 1 & 2 & 2 & 5 \\
\hline
\end{tabular}

\begin{tabular}{|c|c|c|c|c|c|}
\hline \multirow{5}{*}{$\begin{array}{l}\text { How long } \\
\text { have you } \\
\text { been } \\
\text { employed } \\
\text { with Baker } \\
\text { Hughes? }\end{array}$} & & Undetermined & $6 \mathrm{mo}>1 \mathrm{yr}$ & $1 \mathrm{yr}>3 \mathrm{yrs}$ & Total \\
\hline & Latin America & 0 & 1 & 0 & 1 \\
\hline & Middle East & 1 & 0 & 0 & 1 \\
\hline & North America & 0 & 1 & 2 & 3 \\
\hline & Total & 1 & 2 & 2 & 5 \\
\hline
\end{tabular}




\begin{tabular}{|c|c|c|c|c|c|}
\hline \multirow{4}{*}{$\begin{array}{l}\text { How long } \\
\text { have you } \\
\text { been } \\
\text { employed } \\
\text { with Baker } \\
\text { Hughes? }\end{array}$} & & Undetermined & $6 \mathrm{mo}>1 \mathrm{yr}$ & $1 \mathrm{yr}>3 \mathrm{yrs}$ & Total \\
\hline & Eastern & 1 & 0 & 0 & 1 \\
\hline & Western & 0 & 2 & 2 & 4 \\
\hline & Total & 1 & 2 & 2 & 5 \\
\hline
\end{tabular}

\begin{tabular}{|c|c|c|c|c|c|}
\hline \multirow{9}{*}{$\begin{array}{l}\text { How long } \\
\text { have you } \\
\text { been } \\
\text { employed } \\
\text { at your } \\
\text { current } \\
\text { position? }\end{array}$} & & Undetermined & $6 \mathrm{mo}>1 \mathrm{yr}$ & $1 \mathrm{yr}>3 \mathrm{yrs}$ & Total \\
\hline & Latin America & 0 & 1 & 0 & 1 \\
\hline & Middle East & 1 & 0 & 0 & 1 \\
\hline & North America & 0 & 1 & 2 & 3 \\
\hline & Total & 1 & 2 & 2 & 5 \\
\hline & & Undetermined & $6 \mathrm{mo}>1 \mathrm{yr}$ & $1 \mathrm{yr}>3 \mathrm{yrs}$ & Total \\
\hline & Eastern & 1 & 0 & 0 & 1 \\
\hline & Western & 0 & 2 & 2 & 4 \\
\hline & Total & 1 & 2 & 2 & 5 \\
\hline
\end{tabular}

Formal Mentoring $Q 1=$ Yes $Q 2=$ No $Q 3=$ No $Q 4=$ Yes

\begin{tabular}{|c|c|c|c|c|c|}
\hline \multirow{8}{*}{ Gender } & & Undetermined & Male & Female & Total \\
\hline & Middle East & 1 & 0 & 0 & 1 \\
\hline & North America & 0 & 1 & 1 & 2 \\
\hline & Total & 1 & 1 & 1 & 3 \\
\hline & & Undetermined & Male & Female & Total \\
\hline & Eastern & 1 & 0 & 0 & 1 \\
\hline & Western & 0 & 1 & 1 & 2 \\
\hline & & 1 & 1 & 1 & 3 \\
\hline
\end{tabular}




\begin{tabular}{|c|c|c|c|c|}
\hline \multirow{8}{*}{$\begin{array}{c}\text { How long } \\
\text { have you been } \\
\text { employed } \\
\text { with Baker } \\
\text { Hughes? }\end{array}$} & & Undetermined & $1 \mathrm{yr}>3 \mathrm{yrs}$ & Total \\
\hline & Middle East & 1 & 0 & 1 \\
\hline & North America & c & 2 & 2 \\
\hline & Total & 1 & 2 & 3 \\
\hline & & Undetermined & $1 \mathrm{yr}>3 \mathrm{yrs}$ & Total \\
\hline & Eastern & 1 & 0 & 1 \\
\hline & Western & c & 2 & 2 \\
\hline & Total & 1 & 2 & 3 \\
\hline
\end{tabular}

\begin{tabular}{|c|c|c|c|c|}
\hline \multirow{8}{*}{$\begin{array}{l}\text { How long } \\
\text { have you been } \\
\text { employed at } \\
\text { your current } \\
\text { position? }\end{array}$} & & Undetermined & $1 \mathrm{yr}>3 \mathrm{yrs}$ & Total \\
\hline & Middle East & 1 & 0 & 1 \\
\hline & North America & 0 & 2 & 2 \\
\hline & Total & 1 & 2 & 3 \\
\hline & & Undetermined & $1 \mathrm{yr}>3 \mathrm{yrs}$ & Total \\
\hline & Eastern & 1 & 0 & 1 \\
\hline & Western & 0 & 2 & 2 \\
\hline & Total & 1 & 2 & 3 \\
\hline
\end{tabular}

Formal Mentoring $Q 1=$ Yes $Q 2=$ No $Q 3=$ No $Q 4=$ No

\begin{tabular}{|c|c|c|c|c|}
\hline \multirow{7}{*}{ Gender } & & Male & Female & Total \\
\hline & Latin America & 0 & 1 & 1 \\
\hline & North America & 1 & 0 & 1 \\
\hline & Total & 1 & 1 & 2 \\
\hline & & Male & Female & Total \\
\hline & Western & 1 & 1 & 2 \\
\hline & Total & 1 & 1 & 2 \\
\hline
\end{tabular}




\begin{tabular}{|c|c|c|c|}
\hline \multirow{7}{*}{$\begin{array}{l}\text { How long have you } \\
\text { been employed with } \\
\text { Baker Hughes? }\end{array}$} & & $6 \mathrm{mo}>1 \mathrm{yr}$ & Total \\
\hline & Latin America & & 1 \\
\hline & North America & & 1 \\
\hline & Total & & 2 \\
\hline & & $6 \mathrm{mo}>1 \mathrm{yr}$ & Total \\
\hline & Western & & 2 \\
\hline & Total & & 2 \\
\hline
\end{tabular}

\begin{tabular}{|c|c|c|c|}
\hline \multirow{7}{*}{$\begin{array}{c}\text { How long have you } \\
\text { been employed at } \\
\text { your current } \\
\text { position? }\end{array}$} & & $6 \mathrm{mo}>1 \mathrm{yr}$ & Total \\
\hline & Latin America & 1 & 1 \\
\hline & North America & 1 & 1 \\
\hline & Total & 2 & 2 \\
\hline & & $6 \mathrm{mo}>1 \mathrm{yr}$ & Total \\
\hline & Western & 2 & 2 \\
\hline & Total & 2 & 2 \\
\hline
\end{tabular}

Formal Mentoring Q1=No

\begin{tabular}{|l|l|r|r|r|r|}
\hline \multirow{5}{*}{} & \multicolumn{2}{|c|}{ Undetermined } & Male & Female & \multicolumn{1}{|c|}{ Total } \\
\cline { 2 - 7 } & Africa & 0 & 7 & 1 & 8 \\
\cline { 2 - 7 } & Asia Pacific & 0 & 18 & 6 & 24 \\
\cline { 2 - 7 } Gender & Europe & 1 & 3 & 6 & 10 \\
\cline { 2 - 7 } & Latin America & 0 & 14 & 4 & 18 \\
\cline { 2 - 7 } & Middle East & 0 & 22 & 1 & 23 \\
\cline { 2 - 7 } & North America & 0 & 17 & 1 & 18 \\
\cline { 2 - 7 } & Russia \& Caspian & 0 & 14 & 4 & 18 \\
\cline { 2 - 7 } & Total & 1 & 95 & 23 & 119 \\
\cline { 2 - 7 } & \multicolumn{1}{|c|}{ Undetermined } & Male & & Female & Total \\
\cline { 2 - 7 } & Eastern & 1 & 64 & 18 & 83 \\
\cline { 2 - 7 } & Western & 0 & 31 & 5 & 36 \\
\cline { 2 - 7 } & Total & 1 & 95 & 23 & 119 \\
\hline
\end{tabular}




\begin{tabular}{|c|c|c|c|c|c|c|c|c|}
\hline \multirow{13}{*}{$\begin{array}{c}\text { Cow } \\
\text { How } \\
\text { long have } \\
\text { you been } \\
\text { employe } \\
\text { d with } \\
\text { Baker } \\
\text { Hughes? }\end{array}$} & \multicolumn{2}{|c|}{ Undetermined } & \multirow{2}{*}{$\begin{array}{r}<6 \mathrm{mo} \\
0 \\
0\end{array}$} & \multirow{2}{*}{\begin{tabular}{|l}
$\begin{array}{l}6 \mathrm{mo}> \\
1 \mathrm{yr}\end{array}$ \\
2 \\
\end{tabular}} & \multirow{2}{*}{\begin{tabular}{|l}
$\begin{array}{l}1 \mathrm{yr}> \\
3 y r s\end{array}$ \\
4
\end{tabular}} & \multirow{2}{*}{$\begin{array}{l}\begin{array}{l}3 y r s> \\
5 y r s\end{array} \\
\end{array}$} & \multirow{2}{*}{$\begin{array}{l}\begin{array}{l}5 y r s> \\
8 y r s\end{array} \\
\\
\end{array}$} & \multirow{2}{*}{$\begin{array}{r}\text { Total } \\
8 \\
\end{array}$} \\
\hline & Africa & 0 & & & & & & \\
\hline & Asia Pacific & 1 & 4 & 7 & 8 & 4 & 0 & 24 \\
\hline & Europe & 1 & 0 & 6 & 3 & 0 & 0 & 10 \\
\hline & Latin America & 0 & 2 & 4 & 10 & 2 & 0 & 18 \\
\hline & Middle East & 0 & 0 & 6 & 10 & 7 & 0 & 23 \\
\hline & North America & 0 & 4 & 5 & 6 & 3 & 0 & 18 \\
\hline & $\begin{array}{l}\text { Russia \& } \\
\text { Caspian }\end{array}$ & 0 & 1 & 9 & 3 & 4 & 1 & 18 \\
\hline & Total & 2 & 11 & 39 & 44 & 21 & 2 & 119 \\
\hline & Und & & $<6 \mathrm{mo}$ & $\begin{array}{l}6 \mathrm{mo}> \\
1 \mathrm{yr}\end{array}$ & $\begin{array}{l}1 \mathrm{yr}> \\
3 \mathrm{yrs}\end{array}$ & $\begin{array}{l}\text { 3yrs }> \\
5 y r s\end{array}$ & $\begin{array}{l}5 y r s> \\
8 y r s\end{array}$ & Total \\
\hline & Eastern & 2 & 5 & 30 & 28 & 16 & 2 & 83 \\
\hline & Western & 0 & 6 & 9 & 16 & 5 & 0 & 36 \\
\hline & Total & 2 & 11 & 39 & 44 & 21 & 2 & 119 \\
\hline
\end{tabular}

Formal Mentoring $Q 1=$ No $Q 2=$ Yes

\begin{tabular}{|c|c|c|c|c|}
\hline \multirow{11}{*}{ Gender } & & Male & Female & Total \\
\hline & Asia Pacific & 3 & 1 & 4 \\
\hline & Europe & 0 & 1 & 1 \\
\hline & Latin America & 1 & 1 & 2 \\
\hline & Middle East & 6 & 0 & 6 \\
\hline & Russia \& Caspian & 2 & 1 & 3 \\
\hline & Total & 12 & 4 & 16 \\
\hline & & Male & Female & Total \\
\hline & Eastern & 11 & 3 & 14 \\
\hline & Western & 1 & 1 & 2 \\
\hline & Total & 12 & 4 & 16 \\
\hline
\end{tabular}




\begin{tabular}{|c|c|c|c|c|c|c|}
\hline \multirow{11}{*}{$\begin{array}{l}\text { How long } \\
\text { have you } \\
\text { been } \\
\text { employed } \\
\text { with Baker } \\
\text { Hughes? }\end{array}$} & & $<6 \mathrm{mo}$ & $\begin{array}{l}6 \mathrm{mo}> \\
1 \mathrm{yr}\end{array}$ & $\begin{array}{l}1 \mathrm{yr}> \\
3 \mathrm{yrs}\end{array}$ & $\begin{array}{l}\text { 3yrs }> \\
5 y r s\end{array}$ & Total \\
\hline & Asia Pacific & 1 & 1 & 1 & 1 & 4 \\
\hline & Europe & 0 & 1 & 0 & 0 & 1 \\
\hline & Latin America & 0 & 0 & 2 & 0 & 2 \\
\hline & Middle East & 0 & 2 & 3 & 1 & 6 \\
\hline & Russia \& Caspian & 0 & 3 & 0 & 0 & 3 \\
\hline & Total & 1 & 7 & 6 & 2 & 16 \\
\hline & & $<6 \mathrm{mo}$ & $\begin{array}{l}6 \mathrm{mo}> \\
1 \mathrm{yr}\end{array}$ & $\begin{array}{l}1 \mathrm{yr}> \\
3 \mathrm{yrs}\end{array}$ & $\begin{array}{l}\text { 3yrs }> \\
5 y r s\end{array}$ & Total \\
\hline & Eastern & 1 & 7 & 4 & 2 & 14 \\
\hline & Western & 0 & 0 & 2 & 0 & 2 \\
\hline & Total & 1 & 7 & 6 & 2 & 16 \\
\hline
\end{tabular}

\begin{tabular}{|c|c|c|c|c|c|c|}
\hline \multirow{11}{*}{$\begin{array}{c}\text { How long } \\
\text { have you } \\
\text { been } \\
\text { employed } \\
\text { at your } \\
\text { current } \\
\text { position? }\end{array}$} & & $<6 \mathrm{mo}$ & $\begin{array}{l}6 \mathrm{mo}> \\
1 \mathrm{yr}\end{array}$ & $\begin{array}{l}1 \mathrm{yr}> \\
3 \mathrm{yrs}\end{array}$ & $3 y r s>5 y r s$ & Total \\
\hline & Asia Pacific & 1 & 1 & 2 & 0 & 4 \\
\hline & Europe & 0 & 1 & 0 & 0 & 1 \\
\hline & Latin America & 0 & 0 & 2 & 0 & 2 \\
\hline & Middle East & 0 & 3 & 2 & 1 & 6 \\
\hline & Russia \& Caspian & 0 & 3 & 0 & 0 & 3 \\
\hline & Total & 1 & 8 & 6 & 1 & 16 \\
\hline & & $<6 \mathrm{mo}$ & $\begin{array}{l}6 \mathrm{mo}> \\
1 \mathrm{yr}\end{array}$ & $\begin{array}{l}1 \mathrm{yr}> \\
3 \mathrm{yrs}\end{array}$ & $3 \mathrm{yrs}>5 \mathrm{yrs}$ & Total \\
\hline & Eastern & 1 & 8 & 4 & 1 & 14 \\
\hline & Western & 0 & 0 & 2 & 0 & 2 \\
\hline & Total & 1 & 8 & 6 & 1 & 16 \\
\hline
\end{tabular}




\section{Formal Mentoring $Q 1=$ No $Q 2=$ Yes $Q 3=$ Yes}

\begin{tabular}{|c|c|c|c|c|}
\hline \multirow{11}{*}{ Gender } & & Male & Female & Total \\
\hline & Asia Pacific & 2 & 1 & 3 \\
\hline & Europe & 0 & 1 & 1 \\
\hline & Latin America & 1 & 0 & 1 \\
\hline & Middle East & 6 & 0 & 6 \\
\hline & Russia \& Caspian & 2 & 1 & 3 \\
\hline & Total & 11 & 3 & 14 \\
\hline & & Male & Female & Total \\
\hline & Eastern & 10 & 3 & 13 \\
\hline & Western & 1 & 0 & 1 \\
\hline & Total & 11 & 3 & 14 \\
\hline
\end{tabular}

\begin{tabular}{|c|c|c|c|c|c|c|}
\hline \multirow{11}{*}{$\begin{array}{l}\text { How long } \\
\text { have you } \\
\text { been } \\
\text { employed } \\
\text { with Baker } \\
\text { Hughes? }\end{array}$} & & $<6 \mathrm{mo}$ & $\begin{array}{l}6 \mathrm{mo}> \\
1 \mathrm{yr}\end{array}$ & $\begin{array}{l}1 \mathrm{yr}> \\
3 \mathrm{yrs}\end{array}$ & $\begin{array}{l}3 y r s> \\
5 y r s\end{array}$ & Total \\
\hline & Asia Pacific & 1 & 1 & 1 & 0 & 3 \\
\hline & Europe & 0 & 1 & 0 & 0 & 1 \\
\hline & Latin America & 0 & 0 & 1 & 0 & 1 \\
\hline & Middle East & 0 & 2 & 3 & 1 & 6 \\
\hline & Russia \& Caspian & 0 & 3 & 0 & 0 & 3 \\
\hline & Total & 1 & 7 & 5 & 1 & 14 \\
\hline & & $<6 \mathrm{mo}$ & $\begin{array}{l}6 \mathrm{mo}> \\
1 \mathrm{yr}\end{array}$ & $\begin{array}{l}1 \mathrm{yr}> \\
3 \mathrm{yrs}\end{array}$ & $\begin{array}{l}3 y r s> \\
5 y r s\end{array}$ & Total \\
\hline & Eastern & 1 & 7 & 4 & 1 & 13 \\
\hline & Western & 0 & 0 & 1 & 0 & 1 \\
\hline & Total & 1 & 7 & 5 & 1 & 14 \\
\hline
\end{tabular}




\begin{tabular}{|c|c|c|c|c|c|c|}
\hline \multirow{11}{*}{$\begin{array}{l}\text { How long } \\
\text { have you } \\
\text { been } \\
\text { employed } \\
\text { at your } \\
\text { current } \\
\text { position? }\end{array}$} & & $<6 \mathrm{mo}$ & $\begin{array}{l}6 \mathrm{mo}> \\
1 \mathrm{yr} \\
\end{array}$ & $\begin{array}{l}1 \mathrm{yr}> \\
3 \mathrm{yrs}\end{array}$ & $\begin{array}{l}\text { 3yrs }> \\
5 y r s\end{array}$ & Total \\
\hline & Asia Pacific & 1 & 1 & 1 & 0 & 3 \\
\hline & Europe & 0 & 1 & 0 & 0 & 1 \\
\hline & Latin America & 0 & 0 & 1 & 0 & 1 \\
\hline & Middle East & 0 & 3 & 2 & 1 & 6 \\
\hline & Russia \& Caspian & 0 & 3 & 0 & 0 & 3 \\
\hline & Total & 1 & 8 & 4 & 1 & 14 \\
\hline & & $<6 \mathrm{mo}$ & $\begin{array}{l}6 \mathrm{mo}> \\
1 \mathrm{yr}\end{array}$ & $\begin{array}{l}1 \mathrm{yr}> \\
3 \mathrm{yrs}\end{array}$ & $\begin{array}{l}3 \mathrm{yrs}> \\
5 \mathrm{yrs} \\
\end{array}$ & Total \\
\hline & Eastern & 1 & 8 & 3 & 1 & 13 \\
\hline & Western & 0 & 0 & 1 & 0 & 1 \\
\hline & Total & 1 & 8 & 4 & 1 & 14 \\
\hline
\end{tabular}

Formal Mentoring $Q 1=$ No $Q 2=$ Yes $Q 3=$ No

\begin{tabular}{|c|c|c|c|}
\hline \multirow{6}{*}{ Gender } & & Male & Total \\
\hline & Asia Pacific & 1 & 1 \\
\hline & Total & 1 & 1 \\
\hline & & Male & Total \\
\hline & Eastern & 1 & 1 \\
\hline & Total & 1 & 1 \\
\hline
\end{tabular}

\begin{tabular}{|c|l|l|lr|}
\hline \multirow{4}{*}{$\begin{array}{c}\text { How long have you } \\
\text { been employed } \\
\text { with Baker } \\
\text { Hughes? }\end{array}$} & Asia Pacific & 3yrs $>5$ yrs & & Total \\
\cline { 2 - 5 } & Total & & 1 & \\
\cline { 2 - 5 } & & 3yrs $>5$ yrs & & Total \\
\cline { 2 - 5 } & Eastern & & 1 & 1 \\
\cline { 2 - 5 } & Total & & 1 & 1 \\
\hline
\end{tabular}




\begin{tabular}{|c|l|l|lr|}
\hline \multirow{4}{*}{$\begin{array}{c}\text { How long have you } \\
\text { been employed at } \\
\text { your current } \\
\text { position? }\end{array}$} & Asia Pacific & 1yr $>3 \mathrm{yrs}$ & Total \\
\cline { 2 - 5 } & Total & & 1 & \\
\cline { 2 - 5 } & Eastern & $1 \mathrm{yr}>3 \mathrm{yrs}$ & 1 & Total \\
\cline { 2 - 5 } & Total & & 1 & 1 \\
\hline
\end{tabular}

Formal Mentoring $Q 1=$ No $Q 2=$ Yes $Q 3=$ No $Q 4=Y e s$

\begin{tabular}{|l|l|l|lr|}
\hline \multirow{4}{*}{ Gender } & & Male & Total & 1 \\
\cline { 2 - 5 } & Asia Pacific & & 1 & 1 \\
\cline { 2 - 5 } & Total & & 1 & \\
\cline { 2 - 5 } & & Male & Total & 1 \\
\cline { 2 - 5 } & Eastern & & 1 & 1 \\
\cline { 2 - 5 } & Total & & & 1 \\
& & & &
\end{tabular}

\begin{tabular}{|c|l|l|ll|}
\hline \multirow{4}{*}{$\begin{array}{c}\text { How long have you } \\
\text { been employed with } \\
\text { Baker Hughes? }\end{array}$} & Asia Pacific & 3 yrs $>5$ yrs & Total & 1 \\
\cline { 2 - 5 } & Total & & 1 & \\
\cline { 2 - 5 } & & 3 yrs $>5$ yrs & 1 & Total \\
\cline { 2 - 5 } & Eastern & & 1 & 1 \\
\cline { 2 - 5 } & Total & & 1 & 1 \\
\hline
\end{tabular}

How long have you been employed at your current position?

\begin{tabular}{|l|l|lr|} 
& 1 yr $>3 y r s$ & Total & \\
\hline Asia Pacific & & 1 & 1 \\
\hline Total & 1 & & 1 \\
\hline & $1 \mathrm{yr}>3 \mathrm{yrs}$ & Total & 1 \\
\hline Eastern & 1 & & 1 \\
\hline Total & 1 & & 1 \\
\hline
\end{tabular}




\section{Formal Mentoring $Q 1=$ No $Q 2=$ No}

\begin{tabular}{|c|c|c|c|c|}
\hline \multirow{13}{*}{ Gender } & & Male & Female & Total \\
\hline & Africa & 5 & 0 & 5 \\
\hline & Asia Pacific & 9 & 2 & 11 \\
\hline & Europe & 1 & 4 & 5 \\
\hline & Latin America & 9 & 2 & 11 \\
\hline & Middle East & 12 & 1 & 13 \\
\hline & North America & 13 & 1 & 14 \\
\hline & Russia \& Caspian & 6 & 1 & 7 \\
\hline & Total & 55 & 11 & 66 \\
\hline & & Male & Female & Total \\
\hline & Eastern & 33 & 8 & 41 \\
\hline & Western & 22 & 3 & 25 \\
\hline & Total & 55 & 11 & 66 \\
\hline
\end{tabular}

\begin{tabular}{|c|c|c|c|c|c|c|c|c|}
\hline \multirow{13}{*}{$\begin{array}{c}\text { How } \\
\text { long have } \\
\text { you been } \\
\text { employe } \\
\text { d with } \\
\text { Baker } \\
\text { Hughes? }\end{array}$} & \multicolumn{2}{|c|}{ Unspecified } & $<6 \mathrm{mo}$ & $\begin{array}{l}6 \mathrm{mo}> \\
1 \mathrm{yr}\end{array}$ & $\begin{array}{l}1 \mathrm{yr}> \\
3 \mathrm{yrs}\end{array}$ & $\begin{array}{l}3 \mathrm{yrs}> \\
5 \mathrm{yrs}\end{array}$ & $\begin{array}{l}5 \mathrm{yrs}> \\
8 \mathrm{yrs}\end{array}$ & Total \\
\hline & Africa & 0 & 0 & 1 & 2 & 1 & 1 & 5 \\
\hline & Asia Pacific & 1 & 1 & 3 & 4 & 2 & 0 & 11 \\
\hline & Europe & 0 & 0 & 4 & 1 & 0 & 0 & 5 \\
\hline & Latin America & 0 & 1 & 3 & 5 & 2 & 0 & 11 \\
\hline & Middle East & 0 & 0 & 2 & 6 & 5 & 0 & 13 \\
\hline & North America & 0 & 3 & 4 & 5 & 2 & 0 & 14 \\
\hline & Russia \& Caspian & 0 & 1 & 2 & 0 & 3 & 1 & 7 \\
\hline & Total & 1 & 6 & 19 & 23 & 15 & 2 & 66 \\
\hline & \multicolumn{2}{|c|}{ Unspecified } & $<6 \mathrm{mo}$ & $\begin{array}{l}6 \mathrm{mo}> \\
1 \mathrm{yr}\end{array}$ & $\begin{array}{l}1 \mathrm{yr}> \\
3 \mathrm{yrs}\end{array}$ & $\begin{array}{l}3 y r s> \\
5 y r s\end{array}$ & $\begin{array}{l}5 \mathrm{yrs}> \\
8 \mathrm{yrs}\end{array}$ & Total \\
\hline & Eastern & 1 & 2 & 12 & 13 & 11 & 2 & 41 \\
\hline & Western & 0 & 4 & 7 & 10 & 4 & 0 & 25 \\
\hline & Total & 1 & 6 & 19 & 23 & 15 & 2 & 66 \\
\hline
\end{tabular}




\begin{tabular}{|c|c|c|c|c|c|c|}
\hline \multirow{13}{*}{$\begin{array}{l}\text { How long } \\
\text { have you } \\
\text { been } \\
\text { employed } \\
\text { at your } \\
\text { current } \\
\text { position? }\end{array}$} & & $<6 \mathrm{mo}$ & $\begin{array}{l}6 \mathrm{mo}> \\
1 \mathrm{yr}\end{array}$ & $\begin{array}{l}1 \mathrm{yr}> \\
3 \mathrm{yrs}\end{array}$ & $\begin{array}{l}3 y r s> \\
5 y r s\end{array}$ & Total \\
\hline & Africa & 0 & 2 & 3 & 0 & 5 \\
\hline & Asia Pacific & 2 & 3 & 4 & 2 & 11 \\
\hline & Europe & 0 & 4 & 1 & 0 & 5 \\
\hline & Latin America & 2 & 3 & 6 & 0 & 11 \\
\hline & Middle East & 1 & 2 & 8 & 2 & 13 \\
\hline & North America & 6 & 4 & 3 & 1 & 14 \\
\hline & Russia \& Caspian & 1 & 3 & 0 & 3 & 7 \\
\hline & Total & 12 & 21 & 25 & 8 & 66 \\
\hline & & $<6 \mathrm{mo}$ & $\begin{array}{l}6 \mathrm{mo}> \\
1 \mathrm{yr}\end{array}$ & $\begin{array}{l}1 \mathrm{yr}> \\
3 \mathrm{yrs}\end{array}$ & $\begin{array}{l}\text { 3yrs }> \\
5 y r s\end{array}$ & Total \\
\hline & Eastern & 4 & 14 & 16 & 7 & 41 \\
\hline & Western & 8 & 7 & 9 & 1 & 25 \\
\hline & Total & 12 & 21 & 25 & 8 & 66 \\
\hline
\end{tabular}

Formal Mentoring Q1=No Q2=No Q3=Yes

\begin{tabular}{|c|c|c|c|c|}
\hline \multirow{13}{*}{ Gender } & & Male & Female & Total \\
\hline & Africa & 4 & 0 & 4 \\
\hline & Asia Pacific & 9 & 2 & 11 \\
\hline & Europe & 0 & 3 & 3 \\
\hline & Latin America & 8 & 2 & 10 \\
\hline & Middle East & 10 & 1 & 11 \\
\hline & North America & 9 & 1 & 10 \\
\hline & Russia \& Caspian & 5 & 1 & 6 \\
\hline & Total & 45 & 10 & 55 \\
\hline & & Male & Female & Total \\
\hline & Eastern & 28 & 7 & 35 \\
\hline & Western & 17 & 3 & 20 \\
\hline & Total & 45 & 10 & 55 \\
\hline
\end{tabular}




\begin{tabular}{|c|c|c|c|c|c|c|c|c|}
\hline \multirow{13}{*}{$\begin{array}{c}\text { How } \\
\text { long have } \\
\text { you been } \\
\text { employe } \\
\text { d with } \\
\text { Baker } \\
\text { Hughes? }\end{array}$} & \multicolumn{2}{|c|}{ Unspecified } & \multirow{2}{*}{$\begin{array}{r}<6 \mathrm{mo} \\
0\end{array}$} & \multirow{2}{*}{$\begin{array}{l}6 \mathrm{mo}> \\
1 \mathrm{yr} \\
\\
\end{array}$} & \multirow{2}{*}{$\begin{array}{r}1 \mathrm{yr}> \\
3 y r s \\
1 \\
\end{array}$} & \multirow{2}{*}{ 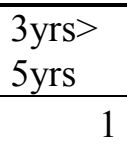 } & \multirow{2}{*}{$\begin{array}{l}\begin{array}{l}\text { 5yrs }> \\
8 y r s\end{array} \\
1 \\
\end{array}$} & \multirow{2}{*}{$\begin{array}{r}\text { Total } \\
4\end{array}$} \\
\hline & Africa & 0 & & & & & & \\
\hline & Asia Pacific & 1 & 1 & 3 & 4 & 2 & 0 & 11 \\
\hline & Europe & 0 & 0 & 3 & 0 & 0 & 0 & 3 \\
\hline & Latin America & 0 & 1 & 3 & 5 & 1 & 0 & 10 \\
\hline & Middle East & 0 & 0 & 1 & 5 & 5 & 0 & 11 \\
\hline & North America & 0 & 2 & 3 & 4 & 1 & 0 & 10 \\
\hline & Russia \& Caspian & 0 & 1 & 2 & 0 & 2 & 1 & 6 \\
\hline & Total & 1 & 5 & 16 & 19 & 12 & 2 & 55 \\
\hline & & & $<6 \mathrm{mo}$ & $\begin{array}{l}6 \mathrm{mo}> \\
1 \mathrm{yr} \\
\end{array}$ & $\begin{array}{l}1 \mathrm{yr}> \\
3 \mathrm{yrs}\end{array}$ & $\begin{array}{l}3 \mathrm{yrs}> \\
5 \mathrm{yrs} \\
\end{array}$ & $\begin{array}{l}5 y r s> \\
8 y r s\end{array}$ & Total \\
\hline & Eastern & 1 & 2 & 10 & 10 & 10 & 2 & 35 \\
\hline & Western & 0 & 3 & 6 & 9 & 2 & 0 & 20 \\
\hline & Total & 1 & 5 & 16 & 19 & 12 & 2 & 55 \\
\hline
\end{tabular}

\begin{tabular}{|c|c|c|c|c|c|c|}
\hline \multirow{13}{*}{$\begin{array}{l}\text { How long } \\
\text { have you } \\
\text { been } \\
\text { employed } \\
\text { at your } \\
\text { current } \\
\text { position? }\end{array}$} & & $<6 \mathrm{mo}$ & $\begin{array}{l}6 \mathrm{mo}> \\
1 \mathrm{yr}\end{array}$ & $\begin{array}{l}1 \mathrm{yr}> \\
3 \mathrm{yrs}\end{array}$ & & Total \\
\hline & Africa & 0 & 2 & 2 & 0 & 4 \\
\hline & Asia Pacific & 2 & 3 & 4 & 2 & 11 \\
\hline & Europe & 0 & 3 & 0 & 0 & 3 \\
\hline & Latin America & 1 & 3 & 6 & 0 & 10 \\
\hline & Middle East & 1 & 1 & 7 & 2 & 11 \\
\hline & North America & 4 & 3 & 3 & 0 & 10 \\
\hline & Russia \& Caspian & 1 & 3 & 0 & 2 & 6 \\
\hline & Total & 9 & 18 & 22 & 6 & 55 \\
\hline & & $<6 \mathrm{mo}$ & $\begin{array}{l}6 \mathrm{mo}> \\
1 \mathrm{yr}\end{array}$ & $\begin{array}{l}1 \mathrm{yr}> \\
3 \mathrm{yrs}\end{array}$ & $\begin{array}{l}3 \mathrm{yrs}> \\
5 \mathrm{yrs}\end{array}$ & Total \\
\hline & Eastern & 4 & 12 & 13 & 6 & 35 \\
\hline & Western & 5 & 6 & 9 & 0 & 20 \\
\hline & Total & 9 & 18 & 22 & 6 & 55 \\
\hline
\end{tabular}


Formal Mentoring Q1=No Q2=No Q3=Yes Q4=Yes

\begin{tabular}{|c|c|c|c|c|}
\hline \multirow{12}{*}{ Gender } & & Male & Female & Total \\
\hline & Africa & 2 & 0 & 2 \\
\hline & Asia Pacific & 3 & 1 & 4 \\
\hline & Latin America & 4 & 0 & 4 \\
\hline & Middle East & 2 & 0 & 2 \\
\hline & North America & 2 & 1 & 3 \\
\hline & Russia \& Caspian & 3 & 0 & 3 \\
\hline & Total & 16 & 2 & 18 \\
\hline & & Male & Female & Total \\
\hline & Eastern & 10 & 1 & 11 \\
\hline & Western & 6 & 1 & 7 \\
\hline & Total & 16 & 2 & 18 \\
\hline
\end{tabular}

\begin{tabular}{|c|c|c|c|c|c|c|c|c|}
\hline \multirow{12}{*}{$\begin{array}{c}\text { How } \\
\text { long } \\
\text { have } \\
\text { you } \\
\text { been } \\
\text { employ } \\
\text { ed with } \\
\text { Baker } \\
\text { Hughes } \\
?\end{array}$} & \multicolumn{2}{|c|}{ Unspecified } & \multirow{2}{*}{$\begin{array}{r}<6 \mathrm{mo} \\
0 \\
\end{array}$} & \multirow{2}{*}{$\begin{array}{l}\begin{array}{l}6 \mathrm{mo}> \\
1 \mathrm{yr}\end{array} \\
\\
\end{array}$} & \multirow{2}{*}{ 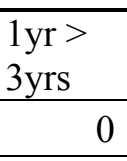 } & \multirow{2}{*}{$\begin{array}{l}\begin{array}{l}3 y r s> \\
5 y r s\end{array} \\
\end{array}$} & \multirow{2}{*}{$\begin{array}{l}\begin{array}{l}5 y r s> \\
8 y r s\end{array} \\
\\
\end{array}$} & \multirow{2}{*}{$\begin{array}{r}\text { Total } \\
2 \\
\end{array}$} \\
\hline & Africa & 0 & & & & & & \\
\hline & Asia Pacific & 1 & 0 & 0 & 2 & 1 & 0 & 4 \\
\hline & Latin America & 0 & 1 & 1 & 1 & 1 & 0 & 4 \\
\hline & Middle East & 0 & 0 & 0 & 0 & 2 & 0 & 2 \\
\hline & North America & 0 & 0 & 0 & 2 & 1 & 0 & 3 \\
\hline & Russia \& Caspian & 0 & 1 & 0 & 0 & 2 & 0 & 3 \\
\hline & Total & 1 & 2 & 2 & 5 & 7 & 1 & 18 \\
\hline & Uns & & $<6 \mathrm{mo}$ & $\begin{array}{l}6 \mathrm{mo}> \\
1 \mathrm{yr}\end{array}$ & $\begin{array}{l}1 \mathrm{yr}> \\
3 \mathrm{yrs}\end{array}$ & $\begin{array}{l}\text { 3yrs }> \\
5 y r s\end{array}$ & $\begin{array}{l}5 y r s> \\
8 y r s\end{array}$ & Total \\
\hline & Eastern & 1 & 1 & 1 & 2 & 5 & 1 & 11 \\
\hline & Western & 0 & 1 & 1 & 3 & 2 & 0 & 7 \\
\hline & Total & 1 & 2 & 2 & 5 & 7 & 1 & 18 \\
\hline
\end{tabular}




\begin{tabular}{|c|c|c|c|c|c|c|}
\hline \multirow{12}{*}{$\begin{array}{l}\text { How long } \\
\text { have you } \\
\text { been } \\
\text { employed } \\
\text { at your } \\
\text { current } \\
\text { position? }\end{array}$} & & $<6 \mathrm{mo}$ & $\begin{array}{l}6 \mathrm{mo}> \\
1 \mathrm{yr}\end{array}$ & $\begin{array}{l}1 \mathrm{yr}> \\
3 \mathrm{yrs}\end{array}$ & $\begin{array}{l}\text { 3yrs }> \\
5 \mathrm{yrs}\end{array}$ & Total \\
\hline & Africa & 0 & 1 & 1 & 0 & 2 \\
\hline & Asia Pacific & 0 & 1 & 2 & 1 & 4 \\
\hline & Latin America & 1 & 1 & 2 & 0 & 4 \\
\hline & Middle East & 1 & 0 & 0 & 1 & 2 \\
\hline & North America & 1 & 0 & 2 & 0 & 3 \\
\hline & Russia \& Caspian & 1 & 1 & 0 & 1 & 3 \\
\hline & Total & 4 & 4 & 7 & 3 & 18 \\
\hline & & $<6 \mathrm{mo}$ & $\begin{array}{l}6 \mathrm{mo}> \\
1 \mathrm{yr}\end{array}$ & $\begin{array}{l}1 \mathrm{yr}> \\
3 \mathrm{yrs}\end{array}$ & $\begin{array}{l}\text { 3yrs }> \\
5 y r s\end{array}$ & Total \\
\hline & Eastern & 2 & 3 & 3 & 3 & 11 \\
\hline & Western & 2 & 1 & 4 & 0 & 7 \\
\hline & Total & 4 & 4 & 7 & 3 & 18 \\
\hline
\end{tabular}

Formal Mentoring $Q 1=$ No $Q 2=$ No $Q 3=$ Yes $Q 4=$ No

\begin{tabular}{|c|c|c|c|c|}
\hline \multirow{13}{*}{ Gender } & & Male & Female & Total \\
\hline & Africa & 2 & 0 & 2 \\
\hline & Asia Pacific & 6 & 1 & 7 \\
\hline & Europe & 0 & 3 & 3 \\
\hline & Latin America & 4 & 2 & 6 \\
\hline & Middle East & 8 & 1 & 9 \\
\hline & North America & 7 & 0 & 7 \\
\hline & Russia \& Caspian & 2 & 1 & 3 \\
\hline & Total & 29 & 8 & 37 \\
\hline & & Male & Female & Total \\
\hline & Eastern & 18 & 6 & 24 \\
\hline & Western & 11 & 2 & 13 \\
\hline & Total & 29 & 8 & 37 \\
\hline
\end{tabular}




\begin{tabular}{|c|c|c|c|c|c|c|c|}
\hline \multirow{13}{*}{$\begin{array}{c}\text { How } \\
\text { long have } \\
\text { you been } \\
\text { employe } \\
\text { d with } \\
\text { Baker } \\
\text { Hughes? }\end{array}$} & & $<6 \mathrm{mo}$ & $\begin{array}{l}6 \mathrm{mo}> \\
1 \mathrm{yr}\end{array}$ & $\begin{array}{l}1 \mathrm{yr}> \\
3 \mathrm{yrs}\end{array}$ & $\begin{array}{l}3 \mathrm{yrs}> \\
5 \mathrm{yrs}\end{array}$ & $\begin{array}{l}5 \mathrm{yrs}> \\
8 \mathrm{yrs}\end{array}$ & Total \\
\hline & Africa & 0 & 0 & 1 & 1 & 0 & 2 \\
\hline & Asia Pacific & 1 & 3 & 2 & 1 & 0 & 7 \\
\hline & Europe & 0 & 3 & 0 & 0 & 0 & 3 \\
\hline & Latin America & 0 & 2 & 4 & 0 & 0 & 6 \\
\hline & Middle East & 0 & 1 & 5 & 3 & 0 & 9 \\
\hline & North America & 2 & 3 & 2 & 0 & 0 & 7 \\
\hline & Russia \& Caspian & 0 & 2 & 0 & 0 & 1 & 3 \\
\hline & Total & 3 & 14 & 14 & 5 & 1 & 37 \\
\hline & & $<6 \mathrm{mo}$ & $\begin{array}{l}6 \mathrm{mo}> \\
1 \mathrm{yr}\end{array}$ & $\begin{array}{l}1 \mathrm{yr}> \\
3 y r s\end{array}$ & $\begin{array}{l}3 \mathrm{yrs}> \\
5 \mathrm{yrs}\end{array}$ & $\begin{array}{l}5 \mathrm{yrs}> \\
8 \mathrm{yrs}\end{array}$ & Total \\
\hline & Eastern & 1 & 9 & 8 & 5 & 1 & 24 \\
\hline & Western & 2 & 5 & 6 & 0 & 0 & 13 \\
\hline & Total & 3 & 14 & 14 & 5 & 1 & 37 \\
\hline
\end{tabular}

\begin{tabular}{|c|c|c|c|c|c|c|}
\hline \multirow{13}{*}{$\begin{array}{l}\text { How long } \\
\text { have you } \\
\text { been } \\
\text { employed } \\
\text { at your } \\
\text { current } \\
\text { position? }\end{array}$} & & $<6 \mathrm{mo}$ & $\begin{array}{l}6 \mathrm{mo}> \\
1 \mathrm{yr}\end{array}$ & $\begin{array}{l}1 \mathrm{yr}> \\
3 \mathrm{yrs}\end{array}$ & $\begin{array}{l}3 \mathrm{yrs}> \\
5 \mathrm{yrs}\end{array}$ & Total \\
\hline & Africa & 0 & 1 & 1 & 0 & 2 \\
\hline & Asia Pacific & 2 & 2 & 2 & 1 & 7 \\
\hline & Europe & 0 & 3 & 0 & 0 & 3 \\
\hline & Latin America & 0 & 2 & 4 & 0 & 6 \\
\hline & Middle East & 0 & 1 & 7 & 1 & 9 \\
\hline & North America & 3 & 3 & 1 & 0 & 7 \\
\hline & Russia \& Caspian & 0 & 2 & 0 & 1 & 3 \\
\hline & Total & 5 & 14 & 15 & 3 & 37 \\
\hline & & $<6 \mathrm{mo}$ & $\begin{array}{l}6 \mathrm{mo}> \\
1 \mathrm{yr}\end{array}$ & $\begin{array}{l}1 \mathrm{yr}> \\
3 \mathrm{yrs}\end{array}$ & $\begin{array}{l}3 \mathrm{yrs}> \\
5 \mathrm{yrs}\end{array}$ & Total \\
\hline & Eastern & 2 & 9 & 10 & 3 & 24 \\
\hline & Western & 3 & 5 & 5 & 0 & 13 \\
\hline & Total & 5 & 14 & 15 & 3 & 37 \\
\hline
\end{tabular}


Formal Mentoring Q1=No Q2=No Q3=No

\begin{tabular}{|c|c|c|c|c|}
\hline \multirow{12}{*}{ Gender } & & Male & Female & Total \\
\hline & Africa & 1 & 0 & 1 \\
\hline & Europe & 1 & 1 & 2 \\
\hline & Latin America & 1 & 0 & 1 \\
\hline & Middle East & 1 & 0 & 1 \\
\hline & North America & 4 & 0 & 4 \\
\hline & Russia \& Caspian & 1 & 0 & 1 \\
\hline & Total & 9 & 1 & 10 \\
\hline & & Male & Female & Total \\
\hline & Eastern & 4 & 1 & 5 \\
\hline & Western & 5 & 0 & 5 \\
\hline & Total & 9 & 1 & 10 \\
\hline
\end{tabular}

\begin{tabular}{|c|c|c|c|c|c|c|}
\hline \multirow{12}{*}{$\begin{array}{l}\text { How long } \\
\text { have you } \\
\text { been } \\
\text { employed } \\
\text { with Baker } \\
\text { Hughes? }\end{array}$} & & $<6 \mathrm{mo}$ & $\begin{array}{l}6 \mathrm{mo}> \\
1 \mathrm{yr}\end{array}$ & $\begin{array}{l}1 \mathrm{yr}> \\
3 \mathrm{yrs}\end{array}$ & $\begin{array}{l}\text { yrs }> \\
5 y r s\end{array}$ & Total \\
\hline & Africa & 0 & 0 & 1 & 0 & 1 \\
\hline & Europe & 0 & 1 & 1 & 0 & 2 \\
\hline & Latin America & 0 & 0 & 0 & 1 & 1 \\
\hline & Middle East & 0 & 0 & 1 & 0 & 1 \\
\hline & North America & 1 & 1 & 1 & 1 & 4 \\
\hline & Russia \& Caspian & 0 & 0 & 0 & 1 & 1 \\
\hline & Total & 1 & 2 & 4 & 3 & 10 \\
\hline & & $<6$ mo & $\begin{array}{l}6 \mathrm{mo}> \\
1 \mathrm{yr}\end{array}$ & $\begin{array}{l}1 \mathrm{yr}> \\
3 \mathrm{yrs}\end{array}$ & $\begin{array}{l}\text { 3yrs }> \\
5 y r s\end{array}$ & Total \\
\hline & Eastern & 0 & 1 & 3 & 1 & 5 \\
\hline & Western & 1 & 1 & 1 & 2 & 5 \\
\hline & Total & 1 & 2 & 4 & 3 & 10 \\
\hline
\end{tabular}




\begin{tabular}{|c|c|c|c|c|c|c|}
\hline \multirow{12}{*}{$\begin{array}{l}\text { How long } \\
\text { have you } \\
\text { been } \\
\text { employed } \\
\text { at your } \\
\text { current } \\
\text { position? }\end{array}$} & & $<6 \mathrm{mo}$ & $\begin{array}{l}6 \mathrm{mo}> \\
1 \mathrm{yr} \\
\end{array}$ & $\begin{array}{l}1 \mathrm{yr}> \\
3 \mathrm{yrs}\end{array}$ & $\begin{array}{l}3 \mathrm{yrs}> \\
5 \mathrm{yrs} \\
\end{array}$ & Total \\
\hline & Africa & 0 & 0 & 1 & 0 & 1 \\
\hline & Europe & 0 & 1 & 1 & 0 & 2 \\
\hline & Latin America & 1 & 0 & 0 & 0 & 1 \\
\hline & Middle East & 0 & 0 & 1 & 0 & 1 \\
\hline & North America & 2 & 1 & 0 & 1 & 4 \\
\hline & Russia \& Caspian & 0 & 0 & 0 & 1 & 1 \\
\hline & Total & 3 & 2 & 3 & 2 & 10 \\
\hline & & $<6 \mathrm{mo}$ & $\begin{array}{l}6 \mathrm{mo}> \\
1 \mathrm{yr}\end{array}$ & $\begin{array}{l}1 \mathrm{yr}> \\
3 \mathrm{yrs}\end{array}$ & $\begin{array}{l}\text { 3yrs }> \\
5 y r s\end{array}$ & Total \\
\hline & Eastern & 0 & 1 & 3 & 1 & 5 \\
\hline & Western & 3 & 1 & 0 & 1 & 5 \\
\hline & Total & 3 & 2 & 3 & 2 & 10 \\
\hline
\end{tabular}

Formal Mentoring $Q 1=$ No $Q 2=$ No $Q 3=$ No $Q 4=$ Yes

\begin{tabular}{|l|l|r|rr|}
\hline \multirow{5}{*}{ Gender } & & Male & Total & 1 \\
\cline { 2 - 5 } & Europe & & 1 & 1 \\
\cline { 2 - 5 } & Middle East & & 1 & 2 \\
\cline { 2 - 5 } & Total & 2 & \\
\cline { 2 - 5 } & & Male & Total & 2 \\
\cline { 2 - 5 } & Eastern & & 2 & 2 \\
\cline { 2 - 5 } & Total & & 2 & \\
\cline { 2 - 5 } & & & & \\
\end{tabular}

How long have you been employed with

Baker Hughes?

\begin{tabular}{|l|r|lr|} 
& 1yr $>3 y r s$ & Total & \\
\hline Europe & 1 & 1 \\
\hline Middle East & 1 & \\
\hline & 2 & \\
\hline & $1 \mathrm{yr}>3 \mathrm{yrs}$ & Total & 2 \\
\hline Eastern & 2 & 2 \\
\hline Total & 2 & 2 \\
\hline
\end{tabular}


How long have you been employed at your current position?

\begin{tabular}{|l|r|lr|} 
& 1yr $>3 y r s$ & Total & 1 \\
\hline Europe & 1 & \\
\hline Middle East & 1 & \\
\hline Total & 2 & \\
\hline & $1 \mathrm{yr}>3 \mathrm{yrs}$ & Total & 2 \\
\hline Eastern & 2 & 2 \\
\hline Total & 2 & \\
\hline
\end{tabular}

Formal Mentoring $Q 1=$ No $Q 2=$ No $Q 3=$ No $Q 4=$ No

\begin{tabular}{|c|c|c|c|c|}
\hline \multirow{11}{*}{ Gender } & & Male & Female & Total \\
\hline & Africa & 1 & 0 & 1 \\
\hline & Europe & 0 & 1 & 1 \\
\hline & Latin America & 1 & 0 & 1 \\
\hline & North America & 4 & 0 & 2 \\
\hline & Russia \& Caspian & 1 & 0 & 1 \\
\hline & Total & 7 & 1 & $\varepsilon$ \\
\hline & & Male & Female & Total \\
\hline & Eastern & 2 & 1 & 3 \\
\hline & Western & 5 & 0 & 5 \\
\hline & Total & 7 & 1 & $\varepsilon$ \\
\hline
\end{tabular}

\begin{tabular}{|c|c|c|c|c|c|c|}
\hline \multirow{10}{*}{$\begin{array}{c}\text { How long } \\
\text { have you } \\
\text { been } \\
\text { employed } \\
\text { with Baker } \\
\text { Hughes? }\end{array}$} & & $<6 \mathrm{mo}$ & $\begin{array}{l}6 \mathrm{mo}> \\
1 \mathrm{yr}\end{array}$ & $\begin{array}{l}1 \mathrm{yr}> \\
5 \mathrm{yrs}\end{array}$ & $\begin{array}{l}3 \mathrm{yrs}> \\
5 \mathrm{yrs}\end{array}$ & Total \\
\hline & Africa & 0 & 0 & 1 & 0 & 1 \\
\hline & Europe & 0 & 1 & 0 & 0 & 1 \\
\hline & Latin America & 0 & 0 & 0 & 1 & 1 \\
\hline & North America & 1 & 1 & 1 & 1 & 4 \\
\hline & Russia \& Caspian & 0 & 0 & 0 & 1 & 1 \\
\hline & Total & 1 & 2 & 2 & 3 & 8 \\
\hline & & $<6 \mathrm{mo}$ & $\begin{array}{l}6 \mathrm{mo}> \\
1 \mathrm{yr}\end{array}$ & $\begin{array}{l}1 \mathrm{yr}> \\
3 \mathrm{yrs}\end{array}$ & $\begin{array}{l}3 \mathrm{yrs}> \\
5 \mathrm{yrs}\end{array}$ & Total \\
\hline & Eastern & 0 & 1 & 1 & 1 & 3 \\
\hline & Western & 1 & 1 & 1 & 2 & 5 \\
\hline
\end{tabular}




\begin{tabular}{|l|l|l|l|l|l|r|}
\hline & Total & 1 & 2 & 2 & 3 & 8 \\
\hline
\end{tabular}

\begin{tabular}{|c|c|c|c|c|c|c|}
\hline \multirow{11}{*}{$\begin{array}{l}\text { How long } \\
\text { have you } \\
\text { been } \\
\text { employed } \\
\text { at your } \\
\text { current } \\
\text { position? }\end{array}$} & & $<6 \mathrm{mo}$ & $\begin{array}{l}6 \mathrm{mo}> \\
1 \mathrm{yr}\end{array}$ & $\begin{array}{l}1 \mathrm{yr}> \\
3 \mathrm{yrs}\end{array}$ & $\begin{array}{l}3 \mathrm{yrs}> \\
5 \mathrm{yrs}\end{array}$ & Total \\
\hline & Africa & 0 & 0 & 1 & 0 & 1 \\
\hline & Europe & 0 & 1 & 0 & 0 & 1 \\
\hline & Latin America & 1 & 0 & 0 & 0 & 1 \\
\hline & North America & 2 & 1 & 0 & 1 & 4 \\
\hline & Russia \& Caspian & 0 & 0 & 0 & 1 & 1 \\
\hline & Total & 3 & 2 & 1 & 2 & 8 \\
\hline & & $<6 \mathrm{mo}$ & $\begin{array}{l}6 \mathrm{mo}> \\
1 \mathrm{yr}\end{array}$ & $\begin{array}{l}1 \mathrm{yr}> \\
3 \mathrm{yrs}\end{array}$ & $\begin{array}{l}3 \mathrm{yrs}> \\
5 \mathrm{yrs}\end{array}$ & Total \\
\hline & Eastern & 0 & 1 & 1 & 1 & 3 \\
\hline & Western & 3 & 1 & 0 & 1 & 5 \\
\hline & Total & 3 & 2 & 1 & 2 & 8 \\
\hline
\end{tabular}


Figure Breakdown of Observed Mentoring Responses - Starting with Positive Q1

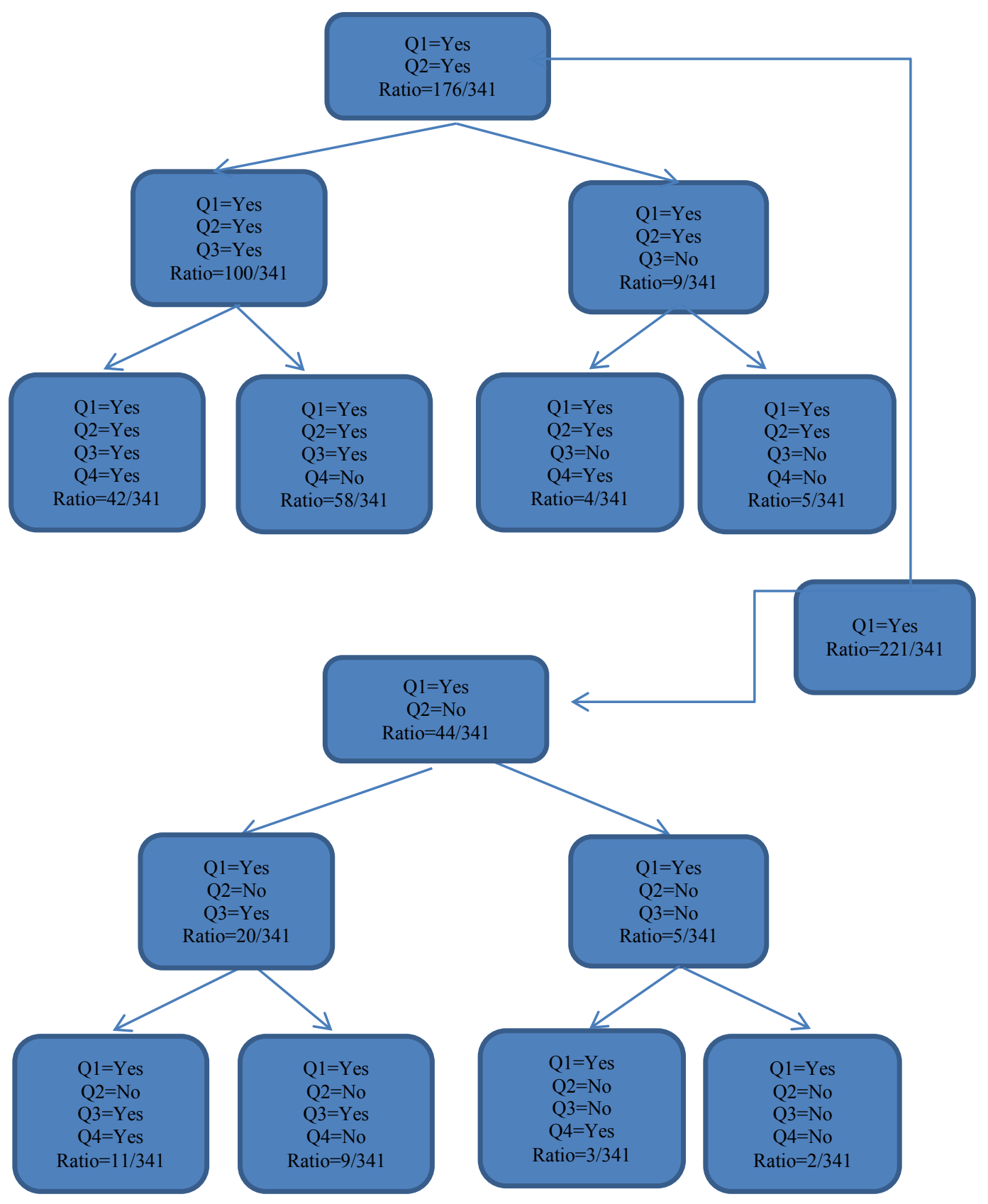


Figure Breakdown of Observed Mentoring Responses - Starting with Negative Q1

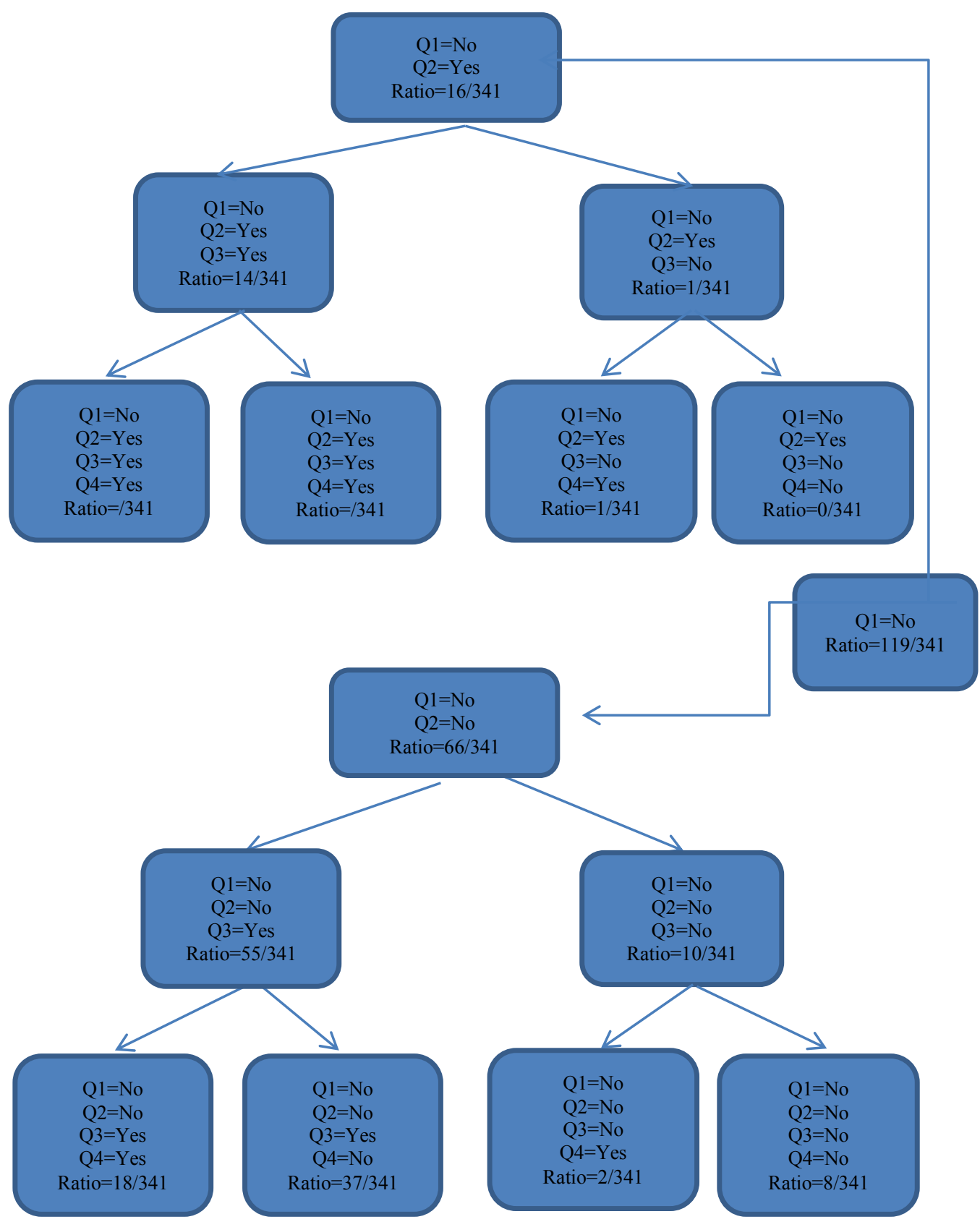




\section{APPENDIX 14}

Respondent vs. NonRespondent

\section{Response vs. NonResponse by Country}

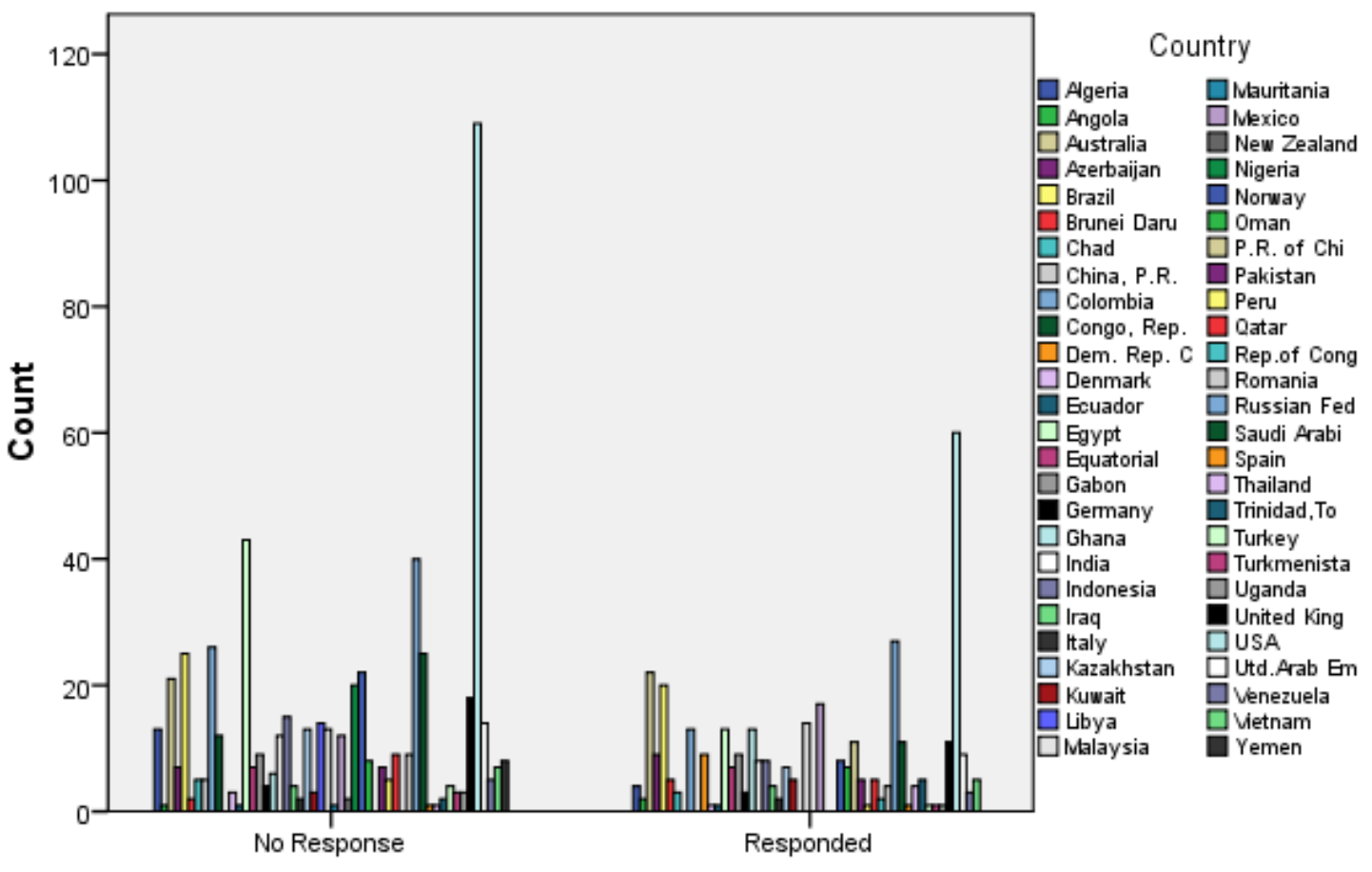


Response vs. NonResponse by Region

\begin{tabular}{|l|c|c|c|c|c|c|c|c|c|}
\hline \multicolumn{8}{|c|}{ Response vs. NonResponse * Region Crosstabulation } \\
\hline & Africa & $\begin{array}{c}\text { Asia } \\
\text { Pacific }\end{array}$ & $\begin{array}{c}\text { Europ } \\
\text { e }\end{array}$ & $\begin{array}{c}\text { Gulf } \\
\text { of } \\
\text { Mexic } \\
\text { o }\end{array}$ & $\begin{array}{c}\text { Latin } \\
\text { Ameri } \\
\text { ca }\end{array}$ & $\begin{array}{c}\text { Middl } \\
\text { e East }\end{array}$ & $\begin{array}{c}\text { North } \\
\text { Ameri } \\
\text { ca }\end{array}$ & $\begin{array}{c}\text { Russia } \\
\text { \& } \\
\text { Caspia } \\
\text { n }\end{array}$ & Total \\
\hline $\begin{array}{l}\text { No } \\
\text { Response }\end{array}$ & 91 & 78 & 63 & 2 & 74 & 121 & 109 & 63 & 601 \\
\hline Responded & 50 & 77 & 31 & 5 & 55 & 59 & 60 & 44 & 381 \\
\hline Total & 141 & 155 & 94 & 7 & 129 & 180 & 169 & 107 & 982 \\
\hline
\end{tabular}

Response vs. NonResponse by Country

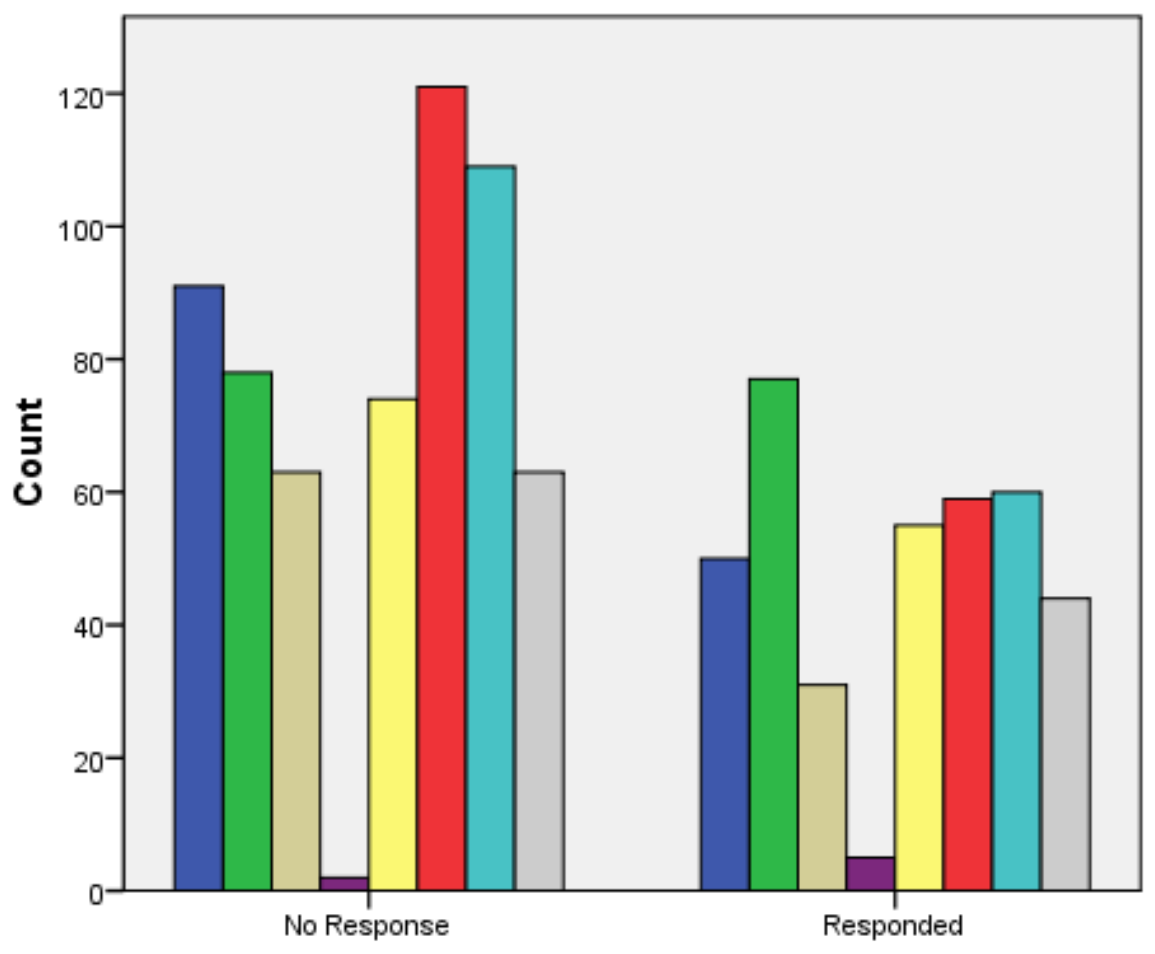

Region
$\square$ Africa
$\square$ Asia Pacifi
$\square$ Europe
$\square$ Gulf of Mex
$\square$ Latin Ameri
$\square$ Middle East
$\square$ North Ameri
$\square$ Russia \& Ca 
Response vs. NonResponse by Hemisphere

\begin{tabular}{|l|c|r|r|}
\hline \multicolumn{4}{|c|}{ Response vs. NonResponse * Hemisphere Crosstabulation } \\
\hline & Eastern & Western & Total \\
\hline No Response & 416 & 185 & 601 \\
\hline Responded & 261 & 120 & 381 \\
\hline Total & 677 & 305 & 982 \\
\hline
\end{tabular}

Response vs. NonResponse by Hemisphere

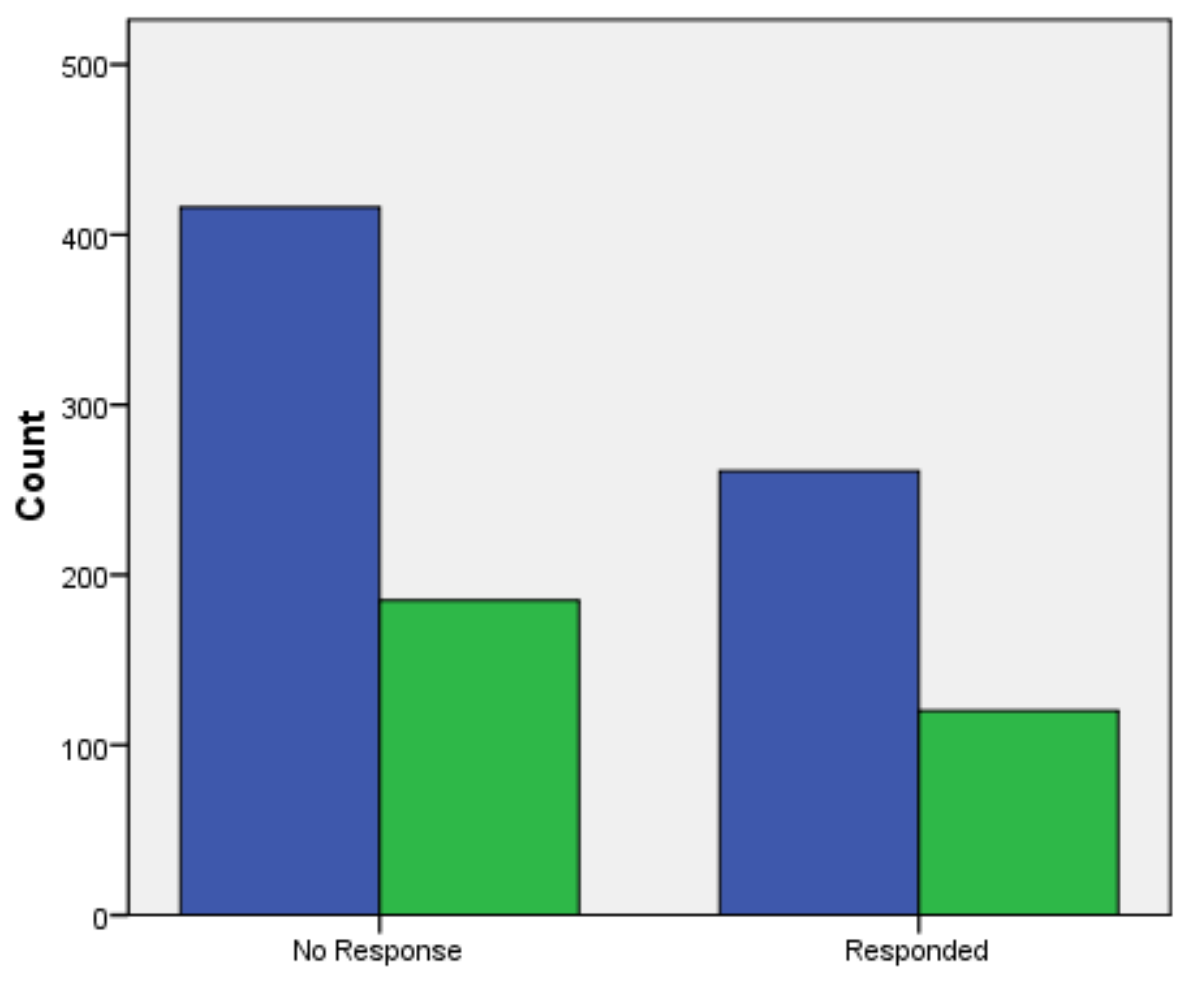

Hemisphere 
VITA

Hanna Bea Hayes

1900 Dartmouth i-4, College Station, TX 77840

Ph.D.: Human Resource Development

Texas A\&M University, College Station, TX 8/2002-5/2012

M.S.: Management

University of Texas at Dallas, Richardson, TX 1/2001-8/2002

B.S.: Journalism/ Recreation, Parks, \& Tourism Sciences

Texas A\&M University, College Station, TX 8/1996-5/2000

Liberty University

Collegiate Faculty Experience

Lynchburg, VA, College of Business

Sept. $10-$ present

- Human Resource Management

South College

Instructor

Asheville, NC, College of Business

- Introduction to Computers

Sept. 09 - Dec. 09

Instructor

Mars Hill College

- Keyboarding I

Mars Hill, NC, College of Business

- Information Technology

University of Nevada (UNLV)

Las Vegas, $N V$, College of Business

- Understanding Business

Blinn College

Bryan, TX, College of Business

- Current Issues in Hospitality

Sept. 09 - Dec. 09

Instructor

- Business Communications Jan. 04 - Sept. 06

Instructor

- Human Resource Management Aug. $02-$ Dec. 03

Instructor

- Web Design/ E-commerce

\section{Research Experience}

EHRD 481 \& 484 - Seminar \& Professional Internship

Jan 2010 - Aug. 2010

- Managed and prepared students to make a smooth transition from an academic environment to a professional business environment. Oversaw professional written and verbal communication with supervisors and co-workers. Managed student's completion of the required assignments with regard to their internship positions.

- Supervised the development of student jobsite documentation and time management skills.

Evaluation of State Tourism Web Sites

Aug 2002 - Sept. 2003

- Conducted a continual two phase evaluation of tourism web sites for twenty states.

Publications \& Presentations

Hayes, H.B., Alagaraja, M. \& Dooley, L.M. (2003). Human capital theory: A reflection on retention of employees in the hospitality industry. Academy of Human Resource Development Proceedings, Minnesota.

Hayes, H.B. (2004). Human capital theory: A reflection on retention of employees in the hospitality industry. Academy of Human Resource Development Conference. Austin, Texas. 